\title{
EQUAÇÖES DIFERENCIAIS ORDINÁRIAS EM ESPAÇOS DE BANACH
}

\author{
Gleiciane da Silva Aragão
}

\author{
DissertaÇão Apresentada \\ AO
}

Instituto de MATEMÁtica e Estatística

DA

Universidade de SÃo Paulo

PARA

Obtenção do Título de Mestre

EM

CIÊNCIAS

Área de Concentração: Matemática Aplicada

Orientador: Prof. Dr. Sergio Muniz Oliva Filho

Durante a elaboração deste trabalho o autor recebeu apoio financeiro do $C N P q$

São Paulo, 31 de agosto de 2006. 


\title{
EQUAÇÕES DIFERENCIAIS ORDINÁRIAS EM ESPAÇOS DE BANACH
}

\author{
Este exemplar corresponde à redação \\ final da dissertação devidamente corrigida \\ e defendida por Gleiciane da Silva Aragão \\ e aprovada pela comissão julgadora.
}

São Paulo, 31 agosto de 2006.

Banca examinadora:

- Prof. Dr. Sergio Muniz Oliva Filho (orientador) - IME-USP

- Prof. Dr. Antônio Luiz Pereira - IME-USP

- Profa. Dra. Márcia Cristina Anderson Braz Federson - ICMC-USP 
Ao meu pai Araldo, à minha mãe Aparecida e ao meu irmão Gleison. 


\section{Agradecimentos}

Antes de qualquer pessoa, gostaria de agradecer a uma que sempre esteve presente direta ou indiretamente em minha vida, fortalecendo-me na minha caminhada. A um "ser divino", a uma "luz", a uma "força maior", que chamo de Deus, o meu muito obrigada por esta dissertação.

Aos meus pais, Araldo e Aparecida, a quem devo o que sou, ao meu irmão Gleison e a todos os meus familiares, meus sinceros agradecimentos.

Ao Prof. Dr. Sergio Muniz Oliva Filho, meu orientador, pela competência, dedicação e sensibilidade com que me auxiliou na elaboração deste trabalho.

Ao Prof. Dr. Antônio Luiz Pereira e à Profa. Dra. Márcia Cristina Federson, pelas sugestões, correções e contribuições a este trabalho.

Ao Prof. Dr. Cláudio Possani, que me ajudou esclarecendo dúvidas, numa parte deste trabalho.

À Luanna e sua família, por terem me acolhido com tanto carinho em sua casa no início do mestrado.

Ao Luís, aos amigos de moradia, Fernando, Adriana e Núbia, aos amigos de Presidente Prudente e região, e a todos os outros, pela compreensão e companherismo.

A todos os colegas que me ajudaram discutindo e esclarecendo dúvidas, entre os quais o Severino e a Andréia.

Aos funcionários da biblioteca do IME-USP, em especial à Elizabeth Barbosa dos 
Santos, que me ajudou na organização da bibliografia deste trabalho.

À Renata, pela ajuda nas correções de Português.

Ao CNPq, pelo apoio financeiro durante a elaboração deste trabalho. 


\section{Resumo}

Neste trabalho lidamos com o problema de valor inicial não linear

$$
\begin{aligned}
& \frac{d x}{d t}=f(t, x), \quad t_{0}<t \leqslant t_{0}+a \\
& x\left(t_{0}\right)=x_{0},
\end{aligned}
$$

em um espaço de Banach $X$, onde $x_{0} \in X$ e $f:\left[t_{0}, t_{0}+a\right] \times X \longrightarrow X$ é uma função contínua. Obtemos condições necessárias para o problema de valor inicial ser bem posto em $X$, isto é, as soluções existem, são únicas e dependem continuamente do dado inicial $\left(t_{0}, x_{0}\right)$. E ainda, estudamos diferenciabilidade das soluções em relação às condições iniciais e existência global de soluções. Finalmente, uma vez que o problema de valor inicial é bem posto no espaço de Banach $X$, analisamos a estabilidade das soluções por primeira aproximação linear. 


\section{Abstract}

In this paper we deal with the nonlinear initial value problem

$$
\begin{aligned}
& \frac{d x}{d t}=f(t, x), \quad t_{0}<t \leqslant t_{0}+a \\
& x\left(t_{0}\right)=x_{0},
\end{aligned}
$$

in a Banach space $X$, where $x_{0} \in X$ and $f:\left[t_{0}, t_{0}+a\right] \times X \longrightarrow X$ is a continuous function. We obtain necessary conditions to the initial value problem to be well posed in $X$, that is, the solutions exist, are unique and depend continuously on their initial data $\left(t_{0}, x_{0}\right)$. In addition, we study differentiability of solutions with respect to initial conditions and global existence of solutions. Finally, since the initial value problem is well posed in the Banach space $X$, we analyse the stability of solutions by first linear approximation. 


\section{Índice}

Agradecimentos $\quad$ iv

Resumo vi

Abstract vii

1 Introdução 1

2 Equações Diferenciais Ordinárias Não Lineares em Espaços de Banach 5

2.1 Alguns Tipos de Soluções para o Problema de Cauchy Não Linear . . 5

2.2 Contra-Exemplos, Existência Local e Unicidade . . . . . . . . . . . 23

2.2.1 Comentários sobre o Teorema de Peano em Dimensão Infinita 38

2.2.2 Unicidade e Dependência Contínua em Dimensão Infinita . . . 50

2.3 Existência Local e Unicidade de Soluções e Dependência Contínua . . 54

2.4 Diferenciabilidade em Relação às Condições Iniciais . . . . . . . . . . 63

2.5 Existência Global . . . . . . . . . . . . . . . . . . . . . . . 74

2.6 Condições de Compacidade . . . . . . . . . . . . . . . . 78

2.6.1 Medida de Não Compacidade e Existência Local . . . . . . . . 78

2.7 Equações Diferenciais Ordinárias em Espaços de Banach Reflexivos . 86

2.7.1 Alguns Resultados e Definições . . . . . . . . . . . . . . . . 87

2.7.2 O Teorema de Existência . . . . . . . . . . . . . . . . . . . 92 
Índice

3 Estabilidade e Instabilidade por Primeira Aproximação Linear $\quad 95$

3.1 Estabilidade e Instabilidade das Soluções da Equação Não Linear . . 97

3.1.1 Estabilidade dos Equilíbrios da Equação Autônoma . . . . . . 106

3.2 Estabilidade por Primeira Aproximação e a Diferencial de Hadamard 107

$\begin{array}{ll}\text { A Apêndice } & 121\end{array}$

A.1 Elementos de Integração . . . . . . . . . . . . . . . . . . . 121

A.2 O Espectro de um Operador Linear . . . . . . . . . . . . . . 123

A.3 Exponencial de um Operador Linear Limitado . . . . . . . . . . . . . 124

$\begin{array}{lr}\text { Referências Bibliográficas } & 126\end{array}$ 


\section{Introdução}

As Equações Diferenciais constituem hoje uma ferramenta importantíssima na modelagem matemática e no estudo de fenômenos naturais relacionados às mais diversas áreas do conhecimento, como a Biologia, Química, Economia, Engenharia, Ecologia e Física. Muitos dos problemas que são modelados por Equações Diferenciais são considerados em espaços de dimensão finita, como por exemplo o $\mathbb{R}^{n}$, o que é muito natural, uma vez que vivemos em um espaço tri-dimensional. Neste caso existe uma ampla teoria desenvolvida em Equações Diferenciais. Por outro lado, alguns problemas envolvendo Equações Diferenciais são considerados em espaços de Banach (espaço vetorial com uma norma definida sobre ele e completo na métrica definida pela norma, resumidamente, espaço normado completo), e estes podem ser de dimensão finita ou infinita.

Neste trabalho estudaremos as Equações Diferenciais Ordinárias em espaços de Banach e, para este estudo, teremos como base os resultados já conhecidos em $\mathbb{R}^{n}$. Tentaremos estender os mesmos para espaços de Banach de qualquer dimensão. Contudo veremos que isso nem sempre será possível, devido ao fato dos espaços de dimensão infinita não possuirem as mesmas propriedades que os de dimensão finita, que são essenciais para construir certos resultados na teoria de Equações Diferenciais Ordinárias. Para tanto utilizaremos a linguagem da Análise Funcional e do Cálculo em Espaços de Banach. Alguns resultados serão dados no apêndice e outros ao longo do trabalho, muitos sem demonstração, apenas citaremos as referências bibliográficas.

Para escrevermos este trabalho, pesquisamos uma ampla bibliografia, composta por artigos e livros. Um longo tempo foi dedicado a esta pesquisa, uma vez que os resultados da teoria de Equações Diferenciais Ordinárias em espaços de Banach se en- 
contravam todos espalhados por diversos artigos e livros. Então buscamos selecionar alguns e reunir da melhor forma possível neste trabalho.

O trabalho está dividido em três capítulos, cujo primeiro é este da introdução, e um apêndice. Nos capítulos seguintes, de um modo geral, nos concentraremos em formular um problema de valor inicial, ou problema de Cauchy, bem posto em um espaço de Banach e explorar o conceito de estabilidade por primeira aproximação linear, com alguns tipos de diferenciais. Para tanto, tentaremos utilizar os resultados já conhecidos em $\mathbb{R}^{n}$. Nesta tentativa, veremos os que continuam ou não sendo válidos quando o espaço de Banach possui dimensão infinita.

No Capítulo 2, estudaremos as Equações Diferenciais Ordinárias não lineares em um espaço de Banach $X$. Mais especificamente o problema de valor inicial não linear

$$
\begin{aligned}
& \frac{d x}{d t}=f(t, x), \quad t_{0}<t \leqslant t_{0}+a \\
& x\left(t_{0}\right)=x_{0},
\end{aligned}
$$

onde $x_{0} \in X$ e $f:\left[t_{0}, t_{0}+a\right] \times X \longrightarrow X$ é uma função contínua, para algum $a>0$. Inicialmente, estamos interessados em estudar a existência de soluções para o problema (1.1), entretanto é necessário definirmos uma noção de solução, ou seja, que tipo de soluções estamos procurando, pois quando $X$ é um espaço de Banach qualquer, temos diferentes noções de soluções uma vez que existem diferentes conceitos de integrais da função $f(t, x)$. Então, investigaremos os tipos de soluções do problema de valor inicial (1.1) utilizando diferentes conceitos de integrais da função $f(t, x)$. Para tanto, teremos como base o trabalho de Cichoń, dado em [5]. Depois fixaremos um tipo de integral para a função $f(t, x)$, que será a integral de Riemann. Assim, uma solução para nós será a solução clássica, então trabalharemos com o problema da existência de soluções clássicas. Na maioria das vezes usaremos somente o termo "solução" para nos referirmos à "solução clássica". Somente nos referiremos ao termo "clássica" se estivermos interessados em estabelecer alguma relação com outros tipos de soluções. Veremos alguns contra-exemplos para mostrar que algumas propriedades clássicas de Equações Diferenciais Ordinárias em $\mathbb{R}^{n}$, ou mais geralmente em qualquer espaço de Banach de dimensão finita, deixam de ser válidas quando o problema de Cauchy (1.1) está sendo considerado em um espaço de Banach $X$ de dimensão infinita, como por exemplo o Teorema de Peano e a extensão de soluções. Estes contra-exemplos foram discutidos por Dieudonné em [12] e Yorke em [38]. Daremos ainda dois resultados importantíssimos que garantem que o Teorema de Peano e que o fato da unicidade de 
soluções implicar dependência contínua só valem em espaços de Banach de dimensão finita. Estes resultados foram demonstrados, respectivamente, por Godunov em [18] e Blasi em [4]. Assim, veremos quais condições serão necessárias para garantir que o problema de Cauchy não linear (1.1) seja bem posto, ou seja, possua existência e unicidade de soluções em espaços de Banach quaisquer, bem como continuidade em relação às condições iniciais $\left(t_{0}, x_{0}\right)$. Veremos também a diferenciabilidade da solução em relação às condições iniciais $\left(t_{0}, x_{0}\right)$ e apresentaremos um conjunto de condições suficientes para a extensão global de soluções. Para tanto utilizaremos [27]. Trabalharemos um pouco com o problema da perda de compacidade nos espaços de dimensão infinita, que é a principal razão para a não validade do Teorema de Peano, utilizando [11]. Finalizaremos o capítulo com um teorema de existência de soluções fracas em espaços de Banach reflexivos, demonstrado por Szép em [36].

No Capítulo 3, terminaremos o nosso trabalho dando uma visão geral do conceito de estabilidade, por primeira aproximação linear, das soluções da equação não linear (1.1), onde a função $f(t, x)$ é continuamente Fréchet diferenciável na variável $x$. Na verdade, exploraremos um conceito mais importante de dependência contínua das soluções de (1.1) em relação ao dado inicial $\left(t_{0}, x_{0}\right)$, uma vez que este fornece informações sobre intervalos de tempo infinito, e o conceito de depedência contínua do Capítlulo 2 somente fornece informações sobre intervalos de tempo finito. Para tanto teremos como base [7]. Finalizaremos com um contra-exemplo para mostrar que estabilidade, por primeira aproximação linear, não vale se $f(t, x)$ for apenas Hadamard diferenciável em $x$. Este contra-exemplo foi discutido por Pereira em [34].

Neste trabalho, estudaremos apenas o caso em que a função $f(t, x)$ é, no mínimo, contínua sobre $\left[t_{0}, t_{0}+a\right] \times X$. Entretanto quando trabalhamos com Equações Diferenciais Parciais, podem aparecer equações do tipo $u_{t}=u_{x x}$, e desta forma os resultados que veremos no Capítulo 2 não podem ser aplicados (com exceção do Teorema 2.3.3), uma vez que o operador derivação pode não ser nem contínuo. Então, neste caso é preciso encontrarmos outra forma de assegurar que um dado problema de valor inicial em um espaço de Banach $X$ seja bem posto. Para tanto é necessário a teoria de Semi-Grupos de Operadores Lineares. Outro problema que aparece quando trabalhamos com Equações Diferenciais Parciais é que o espaço ambiente (onde está $x_{0}$ ) é diferente do espaço de fase (onde está definida a equação dada em (1.1)). Uma linha de estudo que podemos seguir nestes casos é começarmos estudando o problema 
de valor inicial linear

$$
\begin{aligned}
& \frac{d x}{d t}=A x+g(t), \quad t_{0}<t \leqslant t_{0}+a \\
& x\left(t_{0}\right)=x_{0}
\end{aligned}
$$

onde $A: D(A) \longrightarrow X$ é um operador linear com domínio $D(A) \subset X$ e $g:\left[t_{0}, t_{0}+a[\longrightarrow\right.$ $X$, para algum $a>0$, como podemos observar em [23], [25] e [32]. E depois de obtermos que o problema de valor inicial (1.2) é bem posto, perturbamos a equação diferencial homogênea associada a (1.2) (quando $g \equiv 0$ ) com uma função $F(t, x)$ e assim obtemos um problema de valor inicial semi-linear sobre o espaço de Banach $X$, da forma

$$
\begin{aligned}
& \frac{d x}{d t}=A x+F(t, x) \\
& x\left(t_{0}\right)=x_{0} .
\end{aligned}
$$

E este podemos estudar utilizando [23] e [32], onde consideramos que o operador $A$ é o gerador infinitesimal de um $C_{0}$ semi-grupo e de um $C_{0}$ semi-grupo compacto. Nestes dois casos, sob certas condições sobre a função $F(t, x)$, só é possível obtermos soluções clássicas para o problema (1.3) quando o valor inicial $x_{0} \in D(A)$. Quando $x_{0} \in X$, obtemos apenas soluções fracas. Então construímos um espaço de Banach que não é tão "pequeno" quanto $D(A)$, porém não é todo o espaço $X$, e neste é possível obtermos resultados de existência e unicidade de soluções fracas, quando o valor inicial $x_{0}$ pertencer a este espaço. Para tanto fazemos uma "escala" de $X$, e utilizamos os semi-grupos analíticos.

Neste trabalho, não estudaremos os casos descritos no parágrafo anterior. Para mais detalhes, consultar as referências bibliográficas citadas. 
CAPÍtulo 2

\section{Equações Diferenciais Ordinárias}

\section{Não Lineares em Espaços de}

\section{Banach}

\subsection{Alguns Tipos de Soluções para o Problema de Cauchy Não Linear}

Seja $X$ um espaço de Banach real. Consideremos em $X$ o problema de Cauchy ou problema de valor inicial

$$
\begin{aligned}
& \frac{d x}{d t}=f(t, x), \quad t_{0}<t \leqslant t_{0}+a \\
& x\left(t_{0}\right)=x_{0},
\end{aligned}
$$

onde $x_{0} \in X$ e $f: I \times X \longrightarrow X$ é uma função contínua, com $I=\left[t_{0}, t_{0}+a\right]$, para algum $a>0$.

No caso em que $X=\mathbb{R}^{n}$, se $x(t)$ é uma solução da equação diferencial (2.1), então $x(t)$ é uma função continuamente diferenciável sobre o intervalo $I$ e, conseqüentemente, podemos integrar ambos os lados de (2.1) de $t_{0}$ a um arbitrário $t \in I$ e obter

$$
x(t)=x_{0}+\int_{t_{0}}^{t} f(s, x(s)) d s .
$$

Daí, toda solução de (2.1) é também uma solução da equação integral (2.2). Reciprocamente, se $x(t)$ é uma solução contínua de (2.2), definida sobre $I$, e uma vez que 
$f(t, x(t))$ também é contínua, então podemos derivar ambos os lados de (2.2) e obter $\frac{d x(t)}{d t}=f(t, x(t))$. E ainda de (2.2), obtemos $x\left(t_{0}\right)=x_{0}$. Logo $x(t)$ é uma solução da equação diferencial (2.1). Portanto, em $\mathbb{R}^{n}$, é indiferente falarmos em solução da equação integral (2.2) ou diferencial (2.1). Entretanto, em espaços de Banach de dimensão infinita isso não é verdade, pois existem diferentes conceitos de integrais, e cada uma delas dá origem a um "tipo" diferente de solução. Com base nestes fatos, investigaremos os tipos de soluções para o problema (2.1) usando diferentes definições de integrais da função $f(t, x(t))$ e as propriedades destas integrais. Para tanto, teremos como base o trabalho de Cichoń, dado em [5].

Veremos algumas definições de derivadas e de integrais que usaremos para definir as soluções do problema (2.1). Muitos dos conceitos presentes nas definições seguintes se encontram no Apêndice A.1, como por exemplo: conjunto mensurável (Definição A.1.2), medida de um conjunto (Definição A.1.4), função absolutamente contínua- $A C$ (Definição A.1.8), função absolutamente contínua generalizada- $A C G$ e absolutamente contínua generalizada no sentido restrito- $A C G_{*}$ (Definição A.1.9).

Definição 2.1.1 Sejam $x:[a, b] \longrightarrow X$ e $J \subset[a, b]$. Uma função $g: J \longrightarrow X$ é uma pseudo-derivada de $x$ sobre $J$, se para cada $x^{*}$ em $X^{*}$ a função $x^{*} x:[a, b] \longrightarrow \mathbb{R}$ é diferenciável em quase todo $J$ (ver Definição A.1.6) $e\left(x^{*} x\right)^{\prime}=x^{*} g$ em quase todo $J$.

Na Definição 2.1.1, $X^{*}$ denota o espaço dual topológico ou simplesmente o dual do espaço de Banach $X$, isto é, o conjunto de todos os operadores lineares limitados definidos de $X$ em $\mathbb{R}$, ou ainda, o conjunto de todos os funcionais lineares limitados definidos sobre $X$.

Para alguns tipos de soluções precisaremos fixar um $x^{*} \in X^{*}$ e considerar o problema

$$
\left(x^{*} x\right)^{\prime}(t)=x^{*} f(t, x(t)) \quad \text { e } \quad x\left(t_{0}\right)=x_{0}, \quad t \in I .
$$

Estamos interessados em definir derivada aproximada de uma função $x:[a, b] \longrightarrow$ $X$, mas antes precisamos das definições a seguir, que podem ser encontradas com mais detalhes em [33].

Definição 2.1.2 Definimos a densidade de um conjunto $E$ em um ponto c como sendo o limite

$$
\lim _{\mu(N) \rightarrow 0} \frac{\mu(E \cap N)}{\mu(N)}
$$


se este limite existe, onde $N$ denota um intervalo contendo c e $\mu$ é a medida de um conjunto (ver Definição A.1.4).

Notemos que quando $E$ é um intervalo aberto contendo $c$, a densidade de $E$ em $c$ é 1 .

Definição 2.1.3 Dizemos que um número real $L$ é o limite aproximado de uma função $x:[a, b] \longrightarrow X$ em $t_{1}$, se para todo $\epsilon>0$, existe um conjunto $E \subset[a, b]$ de densidade 1 em $t_{1}$ tal que $\|x(t)-L\|<\epsilon$, para todo $t \in E$. Escrevemos

$$
\lim _{t \rightarrow t_{1}} \text { ap } x(t)=L
$$

Assim, temos a seguinte definição:

Definição 2.1.4 Seja $x:[a, b] \longrightarrow X$ e consideremos $t_{1} \in[a, b]$. Definimos $a$ derivada aproximada de $x$ em $t_{1}$, como sendo o limite

$$
\lim _{t \rightarrow t_{1}} a p \frac{x(t)-x\left(t_{1}\right)}{t-t_{1}}=(x)_{a p}^{\prime}\left(t_{1}\right)
$$

onde $(x)_{a p}^{\prime}\left(t_{1}\right)$ denota a derivada aproximada de $x$ em $t_{1}$.

Analogamente, definimos:

Definição 2.1.5 Seja $x:[a, b] \longrightarrow X$ e consideremos $J \subset[a, b]$. Uma função $g: J \longrightarrow X$ é uma pseudo-derivada aproximada de $x$ sobre $J$, se para cada $x^{*} \mathrm{em}$ $X^{*}$ a função $x^{*} x:[a, b] \longrightarrow \mathbb{R}$ é aproximadamente diferenciável em quase todo $J e$ $\left(x^{*} x\right)_{a p}^{\prime}=x^{*} g$ em quase todo $\mathrm{J}$.

Estamos interessados em definir o conceito de diferenciabilidade fraca, mas antes precisamos das definições a seguir.

Definição 2.1.6 Uma seqüência $\left(x_{n}\right)$ em um espaço de Banach $X$ converge fracamente, se existe um elemento $x_{1} \in X$ tal que para todo $x^{*} \in X^{*}$,

$$
\lim _{n \rightarrow \infty} x^{*} x_{n}=x^{*} x_{1}
$$

O elemento $x_{1}$ é chamado o limite fraco de $\left(x_{n}\right)$, e dizemos que $\left(x_{n}\right)$ converge fracamente para $x_{1}$. 
A noção de convergência fraca está discutida com mais detalhes em [26].

Definição 2.1.7 Dizemos que uma função $x:[a, b] \longrightarrow X$ é fracamente contínua em $t_{1} \in[a, b]$, se $t \rightarrow t_{1}$ implicar $x(t) \rightarrow x\left(t_{1}\right)$ na topologia fraca (ver [39], pág. 112, Definição 3). Ou equivalentemente

$$
t \rightarrow t_{1} \Longrightarrow x^{*} x(t) \rightarrow x^{*} x\left(t_{1}\right), \quad \text { para todo } x^{*} \in X^{*}
$$

Então, temos a seguinte definição:

Definição 2.1.8 Dizemos que uma função $x:[a, b] \longrightarrow X$ é fracamente diferenciável em $t_{1} \in[a, b]$, se $\frac{x(t)-x\left(t_{1}\right)}{t-t_{1}}$ converge fracamente, quando $t \rightarrow t_{1}$, para um elemento $x^{\prime}\left(t_{1}\right) \in X$. O elemento $x^{\prime}\left(t_{1}\right)$ é chamado a derivada fraca de $x(t)$ em $t_{1}$.

Observação 1: Uma função fracamente diferenciável é fracamente contínua. De fato, seja $x(t)$ uma função fracamente diferenciável em $t_{1}$, então

$$
\begin{aligned}
\lim _{t \rightarrow t_{1}} x^{*}\left(x(t)-x\left(t_{1}\right)\right) & =\lim _{t \rightarrow t_{1}} x^{*}\left(\frac{x(t)-x\left(t_{1}\right)}{t-t_{1}}\left(t-t_{1}\right)\right)=\lim _{t \rightarrow t_{1}} x^{*}\left(\frac{x(t)-x\left(t_{1}\right)}{t-t_{1}}\right)\left(t-t_{1}\right) \\
& =x^{*} x^{\prime}\left(t_{1}\right)\left(t_{1}-t_{1}\right)=0 .
\end{aligned}
$$

Portanto, $x^{*} x(t) \rightarrow x^{*} x\left(t_{1}\right)$ quando $t \rightarrow t_{1}$, para todo $x^{*} \in X^{*}$. Daí, $x(t)$ é fracamente contínua em $t_{1}$.

A seguir introduziremos novos conceitos de diferencial de uma função $x: A \longrightarrow Z$, onde $A \subset Y$ é aberto, com $Y$ e $Z$ espaços de Banach. Em particular, os resultados que veremos valem quando $A=] a, b[\subset \mathbb{R}$ e os mesmos também serão usados posteriormente. Para mais detalhes consultar [17], [22] e [30].

Definição 2.1.9 Sejam $Y$ e $Z$ espaços de Banach, $A \subset Y$ um subconjunto aberto, $x: A \longrightarrow Z$ e $p \in A$. Dizemos que:

(i) $x$ é Gâteaux diferenciável (G-diferenciável) em $p$ com $G$-diferencial $\delta x(p ; \cdot): Y \longrightarrow Z$ se para cada $h \in Y$ existe o limite

$$
\delta x(p ; h)=\lim _{t \rightarrow 0} \frac{x(p+t h)-x(p)}{t}
$$


(ii) $x$ é Hadamard diferenciável (H-diferenciável) em $p$ com $H$-diferencial $d x(p): Y \longrightarrow Z$ se $d x(p)$ é um operador linear limitado e, para cada $h \in Y$,

$$
\lim _{(t, k) \rightarrow(0,0) \in \mathbb{R} \times Y} \frac{x(p+t h+t k)-x(p)-t d x(p) h}{t}=0 ;
$$

(iii) $x$ é Fréchet diferenciável (F-diferenciável) em $p$ com F-diferencial $d x(p): Y \longrightarrow Z$ se $d x(p)$ é um operador linear limitado e, para cada $h \in Y$,

$$
\lim _{h \rightarrow 0} \frac{x(p+h)-x(p)-d x(p) h}{\|h\|_{Y}}=0 .
$$

Notemos que $F$-diferenciabilidade $\Rightarrow H$-diferenciabilidade $\Rightarrow G$-diferenciabilidade. Por outro lado, a recíproca nem sempre é verdadeira. Quando $Y=\mathbb{R}$ as três diferenciais coincidem.

Analogamente à diferencial em $\mathbb{R}^{n}$, podemos definir as diferenciais de Fréchet de ordem superior e as diferenciais parciais de Fréchet. Essencialmente, as mesmas propriedades válidas para as funções diferenciáveis em $\mathbb{R}^{n}$ são válidas para as funções Fréchet diferenciáveis, tais como: funções Fréchet diferenciáveis são contínuas e deriváveis; soma, produto e multiplicação por escalar de funções Fréchet diferenciáveis são Fréchet diferenciáveis; Regra da Cadeia, Desigualdade do Valor Médio, Teoremas de Taylor, da Função Inversa e Implicita valem.

As diferenciais de Gâteaux e Hadamard são mais "fracas" que a de Fréchet. A diferencial Gâteaux $\delta x(p ; h)$ não satisfaz uma série de propriedades que geralmente valem para as diferenciais, como por exemplo: nem sempre é linear, temos apenas que se $\delta x(p ; h)$ existe e $\alpha \in \mathbb{R}$, então $\delta x(p ; \alpha h)=\alpha \delta x(p ; h)$; mesmo que $\delta x(p ; h)$ exista, isto não implica que $x$ é derivável em $p$; quando $\delta x(p ; h)$ existe e é linear, $x$ não precisa ser contínua em $p$; nem sempre vale a Regra da Cadeia.

Em alguns casos, para mostrarmos que uma função é Fréchet diferenciável, é mais conveniente utilizarmos o resultado da proposição a seguir.

Proposição 2.1.10 Seja $x$ uma função definida em um conjunto $A \subseteq Y$ em $Z$ e seja $p$ um ponto interior de A. Então $x$ é Fréchet diferenciável em $p$ com $F$-diferencial $d x(p)$ se, e somente se

$$
\left\|\frac{x(p+t h)-x(p)-t d x(p) h}{t}\right\|_{Z} \rightarrow 0
$$

quando $t \rightarrow 0$ em $\mathbb{R}$, uniformemente para $h$ na esfera unitária $\left\{h \in Y:\|h\|_{Y}=1\right\}$. 
Finalizaremos com uma proposição, cuja demonstração omitiremos, mas a mesma pode ser encontrada em [22].

Proposição 2.1.11 Sejam $Y, Z, A, x$ e p como na Definição 2.1.9, então temos as seguintes propriedades:

(i) a função $x$ é Hadamard diferenciável em $p$ com $H$-diferencial $d x(p): Y \longrightarrow Z$, se $d x(p)$ é um operador linear limitado e

$$
\left\|\frac{x(p+t h)-x(p)-t d x(p) h}{t}\right\|_{Z} \rightarrow 0,
$$

quando $t \rightarrow 0$, uniformemente para $h$ em qualquer conjunto compacto de $Y$;

(ii) se $Y$ tem dimensão finita, então as diferenciais de Hadamard e Fréchet de $x$ são equivalentes;

(iii) se $x$ é Hadamard diferenciável em $p$, então $x$ é contínua em $p$;

(iv) se $x$ tem uma diferencial de Gâteaux $\delta x(p ; h)$ linear em $h$ e se $x$ é Lipschitziana em uma vizinhança de $p$, então $x$ é Hadamard diferenciável em $p$ e

$$
d x(p) h=\delta x(p ; h) .
$$

Veremos a seguir as definições de algumas integrais. Começaremos com uma breve revisão da integral de Riemann.

Consideremos uma função $g:[a, b] \longrightarrow X, a=t_{0}<t_{1}<\ldots<t_{n}=b$ uma partição de $[a, b]$ e tomemos $\xi_{i} \in\left[t_{i-1}, t_{i}\right]$ para $i=1,2, \ldots, n$. A soma de Riemann é dada por $\sum_{i=1}^{n} g\left(\xi_{i}\right)\left(t_{i}-t_{i-1}\right)$, e a integral de Riemann de $g$ sobre $[a, b]$ é o limite, se este existe, de tal soma quando $\max \left\{\left|t_{i}-t_{i-1}\right|: i=1,2, \ldots, n\right\} \rightarrow 0$. Denotamos este limite por $\int_{a}^{b} g(t) d t$ e dizemos que $g$ é Riemann integrável em $[a, b]$.

Notemos que qualquer ponto $\xi_{i}$, com $i=1,2, \ldots, n$, pode ser associado a qualquer subintervalo $\left[t_{i-1}, t_{i}\right]$, desde que este contenha $\xi_{i}$ e o comprimento seja menor do que $\max \left\{\left|t_{i}-t_{i-1}\right|: i=1,2, \ldots, n\right\}$. Desta forma, temos a mesma restrição de comprimento dos subintervalos para todo $\xi_{i}$, com $i=1,2, \ldots, n$. Por outro lado, podemos considerar o caso em que cada $\xi_{i}$ possua sua própria restrição de comprimento de acordo com a mudança de comportamento da função em cada subintervalo, logo teremos mais variações nos comprimentos dos subintervalos. Onde a função muda 
de comportamento rapidamente, teremos subintervalos de comprimentos "menores" e, onde não muda muito, os comprimentos serão "maiores". Com base nestes fatos obtemos a seguinte generalização da integral de Riemann:

Definição 2.1.12 Uma função $g:[a, b] \longrightarrow X$ é Kurzweil $(K)$ integrável sobre $[a, b]$, se dado $\epsilon>0$, existe uma função $h:[a, b] \longrightarrow X$ e uma função positiva $\delta(\cdot)$ sobre $[a, b]$ tal que para toda partição $\mathscr{P}$ de $[a, b]$ dada por $a=t_{0}<t_{1}<\ldots<t_{n}=b e$ $\xi=\left\{\xi_{1}, \xi_{2}, \ldots, \xi_{n}\right\}$ satisfazendo $\left.\xi_{i} \in\left[t_{i-1}, t_{i}\right] \subset\right] \xi_{i}-\delta\left(\xi_{i}\right), \xi_{i}+\delta\left(\xi_{i}\right)[$ para $i=1,2, \ldots, n$, temos

$$
\left\|h-\sum_{i=1}^{n} g\left(\xi_{i}\right)\left(t_{i}-t_{i-1}\right)\right\|<\epsilon
$$

Escrevemos

$$
\text { (K) } \int_{a}^{b} g(t) d t=h .
$$

A partição $\mathscr{P}$ dada na Definição 2.1.12 é chamada $\delta$-fina. Em particular, quando $\delta$ é uma função constante obtemos a integral de Riemann.

Definição 2.1.13 Uma função $g:[a, b] \longrightarrow X$ é Henstock $(H)$ integrável sobre $[a, b]$, se dado $\epsilon>0$, existe uma função $h:[a, b] \longrightarrow X$ e uma função positiva $\delta(\cdot)$ sobre $[a, b]$ tal que para toda partição $\mathscr{P}$ de $[a, b]$ dada por $a=t_{0}<t_{1}<\ldots<t_{n}=b e$ $\xi=\left\{\xi_{1}, \xi_{2}, \ldots, \xi_{n}\right\}$ satisfazendo $\left.\xi_{i} \in\left[t_{i-1}, t_{i}\right] \subset\right] \xi_{i}-\delta\left(\xi_{i}\right), \xi_{i}+\delta\left(\xi_{i}\right)[$ para $i=1,2, \ldots, n$, temos

$$
\sum_{i=1}^{n}\left\|h\left(t_{i}\right)-h\left(t_{i-1}\right)-g\left(\xi_{i}\right)\left(t_{i}-t_{i-1}\right)\right\|<\epsilon .
$$

Neste caso escrevemos

$$
\text { (H) } \int_{a}^{t} g=h(t)-h(a), \quad t \in[a, b] .
$$

Das Definições 2.1 .12 e 2.1.13, observamos que toda função $(H)$ integrável em $[a, b]$ é $(K)$ integrável em $[a, b]$. Quando $X=\mathbb{R}$ estas duas integrais coincidem.

Podemos obter o Teorema Fundamental do Cálculo, como veremos no teorema a seguir, cuja demonstração pode ser encontrada em [15].

Teorema 2.1.14 Seja $g:[a, b] \longrightarrow X$ uma função contínua. Se g é Fréchet diferenciável em quase todo $[a, b]$, então $g^{\prime} e ́(H)$ integrável sobre $[a, b]$ e

$$
\text { (H) } \int_{a}^{t} g^{\prime}(s) d s=g(t)-g(a), \quad \text { para todo } t \in[a, b] \text {. }
$$


Observação 2: É possível mostrarmos que uma $g:[a, b] \longrightarrow X$ é $(H)$ integrável sobre $[a, b]$ se, e somente se, existe uma função $h:[a, b] \longrightarrow X, A C G_{*}$ (ver Definição A.1.9) e Fréchet diferenciável sobre $[a, b]$ tal que $h^{\prime}=g$ em quase todo $[a, b]$ (ver [19], pág. 147, Teorema 9.17).

Definição 2.1.15 Uma função $g:[a, b] \longrightarrow X$ é fracamente Riemann integrável sobre $[a, b]$, se para qualquer escolha dos pontos $\xi_{i}$ tal que $a \leqslant t_{i-1} \leqslant \xi_{i} \leqslant t_{i} \leqslant b$, com $i=1, \ldots, n$, a soma $\sum_{i=1}^{n} g\left(\xi_{i}\right)\left(t_{i}-t_{i-1}\right)$ converge fracamente para um elemento $h \in X$ quando $\max \left\{\left|t_{i}-t_{i-1}\right|: i=1,2, \ldots, n\right\} \rightarrow 0$. Utilizando a integral de Riemann da função $x^{*} g:[a, b] \longrightarrow \mathbb{R}$, podemos escrever

$$
x^{*} h=\int_{a}^{b} x^{*} g(t) d t, \quad \text { para todo } x^{*} \in X^{*} .
$$

Observação 3: Uma função fracamente contínua é fracamente Riemann integrável. E a integral de uma função fracamente contínua é fracamente diferenciável em relação ao extremo superior de integração e sua Fréchet diferencial é igual ao integrando.

Consideremos uma função $g: \mathbb{R} \longrightarrow X$. Sabemos que faz sentido analisarmos se esta função é Riemann integrável. Entretanto, dificuldades aparecem se $g$ não for contínua, pois lidar com funções descontínuas é uma deficiência da integral de Riemann. A integral de Riemann também possui outras deficiências, sendo que a maior delas são os teoremas de convergência. Portanto, estes fatos nos estimulam a definir uma nova integral, a integral de Lebesgue. No entanto, neste trabalho, esta definição só terá sentido para funções a valores em $\mathbb{R}^{n}$. A extensão da integral de Lebesgue para funções a valores em um espaço de Banach $X$ qualquer, será dada pela integral de Bochner. As definições destas duas integrais, que daremos a seguir, será um pouco diferente das que aparecem na maioria dos trabalhos, no entanto, são equivalentes à estas, como pode ser observado em [29], pág. 222, Apêndice II.

Tomemos o intervalo semi-aberto limitado $J=[a, b[$, onde $a$ e $b$ são números reais. Definimos a função característica de $J$ em $\mathbb{R}$, por

$$
\mathcal{X}_{J}= \begin{cases}1, & \text { se } t \in J \\ 0, & \text { se } t \notin J\end{cases}
$$


Definimos a integral $\int \mathcal{X}_{J}$ como sendo o comprimento do intervalo $J=[a, b[$, então $\int \mathcal{X}_{J}=b-a$.

Definição 2.1.16 Dizemos que $g: \mathbb{R} \longrightarrow \mathbb{R}^{n}$ é uma função patamar, se g é uma combinação linear finita de funções características de intervalos semi-abertos limitados, isto é,

$$
g=\sum_{i=1}^{n} c_{i} \mathcal{X}_{J_{i}}
$$

onde $c_{1}, c_{2}, \ldots, c_{n}$ são coeficientes em $\mathbb{R}^{n}$.

A Definição 2.1 .16 continua sendo a mesma quando $g: \mathbb{R} \longrightarrow X$, onde $X$ é um espaço de Banach qualquer. Entretanto, os coeficientes $c_{1}, c_{2}, \ldots, c_{n}$ são elementos de $X$. Claramente da Definição 2.1.16, a soma de duas funções patamar é uma função patamar e o produto de uma função patamar por um número real é uma função patamar. Em outras palavras, o conjunto de todas as funções patamar é um espaço vetorial. Assumiremos que podemos escolher as funções características $\mathcal{X}_{J_{1}}, \ldots, \mathcal{X}_{J_{n}}$, na representação $(2.4)$, tais que $J_{1}, \ldots, J_{n}$ sejam intervalos semi-abertos limitados disjuntos. Definimos a integral de Lebesgue da função patamar (2.4) por

$$
\text { (L) } \int g=\sum_{i=1}^{n} c_{i} \int \mathcal{X}_{J_{i}} \text {, }
$$

onde $(L) \int g$ denota a integral de Lebesgue da função patamar $g$. Assumiremos que o valor desta integral independe da representação (2.4).

Agora, dada uma função $g: \mathbb{R} \longrightarrow \mathbb{R}^{n}$, podemos escrever

$$
g \cong \sum_{i=1}^{\infty} c_{i} \mathcal{X}_{J_{i}}
$$

onde $\mathcal{X}_{J_{i}}$ é a função característica do intervalo semi-aberto limitado $J_{i}$ e $c_{i} \in \mathbb{R}^{n}$, para todo $i=1,2, \ldots$, se:

$\left.1^{\circ}\right) \quad \sum_{i=1}^{\infty}\left\|c_{i}\right\|_{\mathbb{R}^{n}} \int \mathcal{X}_{J_{i}}<\infty$, onde $\|\cdot\|_{\mathbb{R}^{n}}$ é uma norma em $\mathbb{R}^{n}$;

$\left.2^{\mathrm{o}}\right) \quad g(t)=\sum_{i=1}^{\infty} c_{i} \mathcal{X}_{J_{i}}(t)$ nos pontos $t$ em que a série é absolutamente convergente.

Definição 2.1.17 Dizemos que uma função $g: \mathbb{R} \longrightarrow \mathbb{R}^{n}$ é Lebesgue (L) integrável, se existem intervalos semi-abertos limitados $J_{i} \subset \mathbb{R}$ e coeficientes $c_{i} \in \mathbb{R}^{n}, i=1,2, \ldots$, 
tais que vale (2.5), ou seja, $1^{\circ}$ ) e $2^{\circ}$ ) são satisfeitos. Denotamos a integral de Lebesgue de $g$ por $(L) \int g(t) d t$ e definimos como sendo a soma

$$
\text { (L) } \int g(t) d t=\sum_{i=1}^{\infty} c_{i} \int \mathcal{X}_{J_{i}} .
$$

A integral $(L) \int g(t) d t$ é determinada unicamente como pode ser observado em [29], pág. 3, Teorema 3.1.

Observação 4: É possível mostrarmos que uma função $g:[a, b] \longrightarrow \mathbb{R}^{n}$ é $(L)$ integrável sobre $[a, b]$ se, e somente se, existe uma função $h:[a, b] \longrightarrow \mathbb{R}^{n}, A C$ (ver Definição A.1.8) e Fréchet diferenciável tal que $h^{\prime}=g$ em quase todo $[a, b]$ (ver [19], pág. 61, Teorema 4.15).

De maneira análoga à definição da integral de Lebesgue, podemos definir a integral de Bochner de uma função $g: \mathbb{R} \longrightarrow X$, onde $X$ é um espaço de Banach qualquer, lembrando apenas que, na representação (2.5), $c_{i} \in X$ para todo $i=1,2, \ldots$ e em $1^{\circ}$ ) a norma em $\mathbb{R}^{n}$ é substituída pela norma em $X$.

Definição 2.1.18 Dizemos que uma função $g: \mathbb{R} \longrightarrow X$ é Bochner (B) integrável, se existem intervalos semi-abertos limitados $J_{i} \subset \mathbb{R}$ e coeficientes $c_{i} \in X, i=1,2, \ldots$, tais que vale (2.5), ou seja, $\left.1^{\circ}\right)$ e $\left.2^{\circ}\right)$ são satisfeitos. Denotamos a integral de Bochner de $g$ por $(B) \int g(t) d t$ e definimos como sendo a soma

$$
\text { (B) } \int g(t) d t=\sum_{i=1}^{\infty} c_{i} \int \mathcal{X}_{J_{i}} \text {. }
$$

Em particular, a Definição 2.1.18 vale para $g$ definida em um subconjunto $[a, b]$ de $\mathbb{R}$. Essencialmente, as propriedades que são válidas para outros tipos de integrais são válidas para as integrais de Bochner. Em particular, no teorema a seguir, veremos que, para as funções $(B)$ integráveis, também podemos obter o Teorema Fundamental do Cálculo (ver [29], pág. 168, Teorema 2.3).

Teorema 2.1.19 Sejam $g:[a, b] \longrightarrow X$ uma função contínua em $] a, b[$ e $F$ uma função contínua em $[a, b]$ tais que $F^{\prime}=g$ em $] a, b\left[\right.$. Então $(B) \int_{a}^{b} g(t) d t$ existe $e$ ainda

$$
\text { (B) } \int_{a}^{b} g(t) d t=F(b)-F(a) \text {. }
$$


Veremos, a seguir, a definição da integral de Pettis.

Definição 2.1.20 Uma função $g:[a, b] \longrightarrow X$ é Pettis $(P)$ integrável se:

(i) para todo $x^{*} \in X^{*}, x^{*} g:[a, b] \longrightarrow \mathbb{R}$ é (L) integrável sobre $[a, b]$;

(ii) para todo $J \subset[a, b], J$ mensurável (ver Definição A.1.2), existe $h \in X$ tal que para todo $x^{*} \in X^{*}, x^{*} h=(L) \int_{J} x^{*} g(s) d s$. Denotamos por

$$
h=(P) \int g(t) d t .
$$

Daremos uma definição de integral, que é uma generalização para as integrais Pettis e Henstock. Para mais detalhes, consultar [6].

Definição 2.1.21 Uma função $g:[a, b] \longrightarrow X$ é Henstock-Pettis (HP) integrável, se existe uma função $h:[a, b] \longrightarrow X$ com as seguintes propriedades:

(i) para todo $x^{*} \in X^{*}, x^{*} g:[a, b] \longrightarrow \mathbb{R}$ é (H) integrável sobre $[a, b]$;

(ii) para todo $t \in[a, b]$ e todo $x^{*} \in X^{*}$, temos $x^{*} h(t)=(H) \int_{0}^{t} x^{*} g(s) d s$.

Denotamos por

$$
h=(H P) \int g(t) d t .
$$

Definição 2.1.22 Uma função $g:[a, b] \longrightarrow X$ é Denjoy (D) integrável sobre $[a, b]$, se existe uma função $A C G$ (ver Definição A.1.9), $h:[a, b] \longrightarrow X$ tal que $h$ é aproximadamente diferenciável em quase todo $[a, b]$ e $(h)_{a p}^{\prime}=g$ em quase todo $[a, b]$. Denotamos por

$$
h=(D) \int g(t) d t
$$

A integral de Denjoy é uma generalização da caracterização da integral de Lebesgue dada na Observação 4.

Definição 2.1.23 Uma função $g:[a, b] \longrightarrow X$ é Denjoy-Pettis (DP) integrável sobre $[a, b]$, se para cada $x^{*} \in X^{*}$ a função $x^{*} g:[a, b] \longrightarrow \mathbb{R}$ é $(D)$ integrável sobre $[a, b] e$ se para todo intervalo $J \subset[a, b]$ existe um elemento $z_{J} \in X$ tal que

$$
x^{*} z_{J}=(D) \int_{J} x^{*} g(t) d t, \quad \text { para todo } x^{*} \in X^{*} .
$$


Apresentaremos, a seguir, um diagrama relacionando às definições das integrais dadas, no qual $(Y) \Longrightarrow(Z)$ significa que se uma função é $(Y)$ integrável, então é $(Z)$ integrável.

$$
\begin{aligned}
& (B) \Longrightarrow(H) \Longrightarrow(H P) \Longleftarrow(P) \\
& \Downarrow \quad \Downarrow \quad \Downarrow \quad \Downarrow \\
& (P) \quad(D)====\Longrightarrow(D P)
\end{aligned}
$$

Estas implicações fornecerão algumas conseqüências para os tipos de soluções do problema de valor inicial (2.1).

Com base nas definições anteriores já podemos definir alguns tipos de soluções para o problema (2.1).

Definição 2.1.24 Uma função $x: I \longrightarrow X$ é uma solução pseudo-Henstock do problema de Cauchy (2.1), se satisfaz as seguintes condições:

(i) $x(\cdot)$ é fracamente $A C G_{*}$, isto é, para todo $x^{*} \in X^{*}, x^{*} x$ é $A C G_{*}$;

(ii) $x\left(t_{0}\right)=x_{0}$;

(iii) para cada $x^{*} \in X^{*}$ existe um conjunto $A\left(x^{*}\right)$ com medida de Lebesgue nula (ver Definição A.1.5) tal que para cada $t \notin A\left(x^{*}\right), x^{*} x^{\prime}(t)=x^{*} f(t, x(t))$, onde a derivada considerada é a pseudo-derivada.

Definição 2.1.25 Uma função $x: I \longrightarrow X$ é uma solução pseudo-Denjoy do problema de Cauchy (2.1), se satisfaz as seguintes condições:

(i) $x(\cdot)$ é fracamente $A C G$, isto é, para cada $x^{*} \in X^{*}, x^{*} x$ é $A C G$;

(ii) $x\left(t_{0}\right)=x_{0}$;

(iii) para cada $x^{*} \in X^{*}$ existe um conjunto $A\left(x^{*}\right)$ com medida de Lebesgue nula tal que para cada $t \notin A\left(x^{*}\right),\left(x^{*} x\right)_{a p}^{\prime}(t)=x^{*} f(t, x(t))$.

Contudo, não existem somente estas duas definições de soluções que acabamos de ver. É possível obtermos outras noções de soluções para o problema (2.1), como veremos na Definição 2.1.26, desde que a função $x: I \longrightarrow X$ satisfaça hipóteses de continuidade e diferenciabilidade, tais como uma das dadas a seguir. 
(I) Hipóteses de continuidade:

(a) $x$ é $A C$;

(b) $x$ é $A C G_{*}$;

(c) $x$ é $A C G$;

(d) $x$ é fracamente $A C G_{*}$;

(e) $x$ é fracamente $A C G$.

(II) Hipóteses de diferenciabilidade:

(a) $x$ é continuamente diferenciável $\left(x \in C^{1}\right)$;

(b) $x$ é fracamente diferenciável;

(c) $x$ é diferenciável em quase todo $t$;

(d) $x$ é pseudo-diferenciável;

(e) $x$ é aproximadamente diferenciável em quase todo $t$;

(f) $x$ é aproximadamente pseudo-diferenciável.

Com base nas hipóteses acima temos:

Definição 2.1.26 Uma função $x: I \longrightarrow X$ é uma solução do problema de valor inicial (2.1) do tipo:

(a) clássica se satisfaz (Ia) e (IIa);

(b) fraca se satisfaz (Ia) e (IIb);

(c) Carathéodory se satisfaz (Ia) e (IIc);

(d) pseudo se satisfaz (Ia) e (IId);

(e) Henstock se satisfaz (Ib) e (IIc);

(f) pseudo-Henstock se satisfaz (Id) e (IId);

(g) Denjoy se satisfaz (Ic) e (IIe);

(h) pseudo-Denjoy se satisfaz (Ie) e (IIf).

Juntamente com a condição inicial $x\left(t_{0}\right)=x_{0}$. E a derivada de $x$ assumida no sentido de (II) satisfaz a equação $x^{\prime}=f(t, x)$ (para cada $t$ no caso (IIa), em quase todo $t$ nos casos (IIC) e (IIe)) ou satisfaz (2.3) nos casos (IIb), (IId) e (IIf).

Fixando um tipo de solução e uma determinada definição de integral da função contínua $f(t, x(t))$, digamos $f(t, x(t))$ é $(Y)$ integrável, onde $(Y)$ corresponde a um 
tipo de integração que especificaremos depois, o problema de Cauchy (2.1) é equivalente ao problema integral

$$
x(t)=x_{0}+(Y) \int_{t_{0}}^{t} f(s, x(s)) d s, \quad t \in\left[t_{0}, t_{0}+a\right] .
$$

Veremos, a seguir, que cada solução do problema (2.1) é equivalente a uma solução do problema integral (2.7), com a integral dependendo do tipo de solução.

Teorema 2.1.27 Toda solução $x$ do problema (2.1) é equivalente a uma solução y da equação integral (2.7) nos seguintes casos:

(a) $x$ é solução clássica: a integral de Riemann;

(b) $x$ é solução fraca: a integral de Riemann fraca;

(c) $x$ é solução de Carathéodory: a integral de Bochner;

(d) $x$ é solução pseudo: a integral de Pettis;

(e) $x$ é solução de Henstock: a integral de Henstock;

(f) $x$ é solução pseudo-Henstock: a integral de Henstock-Pettis;

(g) $x$ é solução de Denjoy: a integral de Denjoy;

(h) $x$ é solução pseudo-Denjoy: a integral de Denjoy-Pettis.

\section{Demonstração:}

(a) Se $x$ é uma solução clássica de (2.1), então $x^{\prime}(t)=f(t, x(t))$ e $x\left(t_{0}\right)=x_{0}$, para todo $t \in I$. Uma vez que $x$ é continuamente diferenciável, então $x^{\prime}(t)=f(t, x(t))$ é contínua. Logo Riemann integrável de $t_{0}$ a um arbitrário $t \in I$. Aplicando o Teorema Fundamental do Cálculo, temos

$$
x(t)-x\left(t_{0}\right)=\int_{t_{0}}^{t} x^{\prime}(s) d s=\int_{t_{0}}^{t} f(s, x(s)) d s \Longrightarrow x(t)=x_{0}+\int_{t_{0}}^{t} f(s, x(s)) d s .
$$

Portanto, $x$ é uma solução da equação integral (2.7). Reciprocamente, se $y$ é uma solução de (2.7), onde a integral considerada é a de Riemann, então

$$
y(t)=x_{0}+\int_{t_{0}}^{t} f(s, y(s)) d s .
$$

Como $f(t, y(t))$ é Riemann integrável e contínua em $I$, então $y$ é $A C$. E ainda, de (2.8) temos $y\left(t_{0}\right)=x_{0}$ e $y^{\prime}(t)=f(t, x(t))$, logo $y \in C^{1}$. Portanto, $y$ é uma solução clássica de (2.1). 
Notemos que $A_{-1} \equiv A$ em $H_{p,\{\mathcal{B}\}}^{2}(\Omega)$, e assim $A_{-1}$ é uma extensão do operador $A$.

Consideremos o seguinte diagrama

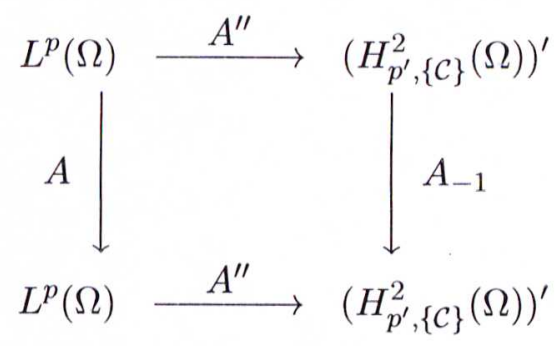

Afirmamos que o diagrama comuta. De fato, se $u \in L^{p}(\Omega)$ então

$$
\begin{gathered}
A^{\prime \prime} \circ \underbrace{A^{-1} u}_{\in H_{p,\{\mathcal{B}\}}^{2}}=A \circ A^{-1} u=u . \\
\Rightarrow\left(A^{\prime \prime}\right)^{-1} \circ A^{\prime \prime} \circ A^{-1} u=\left(A^{\prime \prime}\right)^{-1} u \Rightarrow A^{-1} u=\left(A^{\prime \prime}\right)^{-1} u, \quad \forall u \in L^{p}(\Omega) .
\end{gathered}
$$

Portanto,

$$
\left(A^{\prime \prime}\right)^{-1} u \in D(A)=H_{p,\{\mathcal{B}\}}^{2}(\Omega), \quad \forall u \in L^{p}(\Omega) .
$$

Logo, $A^{\prime \prime} \circ A \circ A^{\prime \prime-1}$ está bem definido em $L^{p}(\Omega)=D\left(A_{-1}\right)$. E ainda,

$$
A^{\prime \prime} \circ A \circ \underbrace{\left(A^{\prime \prime}\right)^{-1} u}_{\in H_{p,\{\mathcal{B}\}}^{2}}=A^{\prime \prime} \circ A^{\prime \prime} \circ\left(A^{\prime \prime}\right)^{-1} u=A^{\prime \prime} \underbrace{u}_{\in L^{p}}=A_{-1} u, \quad \forall u \in L^{p}(\Omega) .
$$

Então,

$$
A_{-1} \equiv A^{\prime \prime} \circ A \circ\left(A^{\prime \prime}\right)^{-1}
$$

e o diagrama comuta. Agora, $A_{-1}$ é um operador linear, fechado e densamente definido, uma vez que $D\left(A_{-1}\right)=L^{p}(\Omega)$ é denso em $\left(H_{p^{\prime},\{\mathcal{C}\}}^{2}(\Omega)\right)^{\prime}$. Veremos ainda na proposição a seguir que o operador $A_{-1}$ é setorial.

Proposição 2.1.28 $A_{-1}$ é um operador setorial em $\left(H_{p^{\prime},\{\mathcal{C}\}}^{2}(\Omega)\right)^{\prime}, \operatorname{com} \rho(A)=\rho\left(A_{-1}\right)$. E ainda, dado $\theta \geqslant 0$, se definirmos

$$
X_{-1}^{\theta}=D\left(A_{-1}^{\theta}\right)
$$

então $A_{-1}$ também é um operador setorial em $X_{-1}^{\theta}$, que denotaremos por $A_{\theta-1}$.

\section{Prova:}


Daí, $y$ é $A C$. Agora, de (2.9) temos $\left(x^{*} y\right)^{\prime}(t)=x^{*} f(t, y(t))$ para todo $x^{*} \in X^{*}$. Logo $y$ é fracamente diferenciável. E ainda, $x^{*} y\left(t_{0}\right)=x^{*} x_{0}$, isto é, $x^{*}\left(y\left(t_{0}\right)-x_{0}\right)=0$ para todo $x^{*} \in X^{*}$. Usando um corolário do Teorema de Hahn-Banach (ver [26], pág. 223, Corolário 4.3-4), obtemos $y\left(t_{0}\right)=x_{0}$. Portanto, $y$ é uma solução fraca de (2.1).

(c) Se $x$ é uma solução de Carathéodory de (2.1), então $x$ é $A C$ e diferenciável em quase todo $t \in I$, com $x^{\prime}(t)=f(t, x(t))$ em quase todo $t \in I$ e $x\left(t_{0}\right)=x_{0}$. Como $f(t, x(t))$ é contínua em $I$ e $x$ é $A C$ em $I, \operatorname{com} x^{\prime}(t)=f(t, x(t))$ em quase todo $t \in I$, então, pelo Teorema 2.1.19, existe a integral $(B) \int_{t_{0}}^{t} f(s, x(s)) d s$, para um arbitrário $t \in I$. E ainda,

$$
\text { (B) } \int_{t_{0}}^{t} f(s, x(s)) d s=x(t)-x\left(t_{0}\right) \Longrightarrow x(t)=x_{0}+(B) \int_{t_{0}}^{t} f(s, x(s)) d s .
$$

Portanto, $x$ é uma solução da equação integral (2.7). Reciprocamente, se $y$ é uma solução da equação integral (2.7), onde a integral considerada é a de Bochner, então

$$
y(t)=x_{0}+(B) \int_{t_{0}}^{t} f(s, y(s)) d s .
$$

Em vez de mostrarmos que $y$ é $A C$, por simplificação, mostraremos que $y$ é contínua. De fato, inicialmente podemos estender a definição da função $f(t, y(t))$ para todo $\mathbb{R}$, tomando $f(t, y(t))=0$ para $t \notin I$. Desta forma, $f(t, y(t))$ é $(B)$ integrável sobre $\mathbb{R}$. Definindo $F(t)=y(t)-x_{0}$, mostraremos que $F$ é contínua. Para isto, basta provarmos que $F\left(t+h_{n}\right)-F(t) \rightarrow 0$ quando $h_{n} \rightarrow 0$. De (2.10) temos

$$
\begin{aligned}
F\left(t+h_{n}\right)-F(t) & =(B) \int_{t_{0}}^{t+h_{n}} f(s, y(s)) d s-(B) \int_{t_{0}}^{t} f(s, y(s)) d s \\
& =(B) \int_{t}^{t+h_{n}} f(s, y(s)) d s=(B) \int_{I_{n}} f(s, y(s)) d s,
\end{aligned}
$$

onde $I_{n}=\left[t, t+h_{n}\right]$. Logo, se $h_{n} \rightarrow 0$ então o comprimento de $I_{n}$ tende a zero, e daí $(B) \int_{I_{n}} f(s, y(s)) d s \rightarrow 0$ (ver [29], pág. 164, Teorema 1.1). Assim,

$$
F\left(t+h_{n}\right)-F(t) \rightarrow 0, \quad \text { quando } h_{n} \rightarrow 0
$$

Portanto $F(t)$ é contínua em $I$ e, conseqüentemente, $y(t)$ é contínua em $I$. Com algumas modificações, mostramos que $y(t)$ é $A C$ em $I$. Logo é diferenciável em quase todo $t \in I$. E ainda, de (2.10) temos $y\left(t_{0}\right)=x_{0}$. 
Falta mostrarmos que $y^{\prime}(t)=f(t, y(t))$. Definimos novamente $F(t)=y(t)-x_{0}$ e tomamos $t$ e $t+h$ em $I$. De (2.10) obtemos

$$
\frac{F(t+h)-F(t)}{h}=\frac{1}{h}(B) \int_{I_{h}} f(s, y(s)) d s,
$$

onde $I_{h}=[t, t+h]$. Logo,

$$
\left\|\frac{F(t+h)-F(t)}{h}-f(t, y(t))\right\| \leqslant \frac{1}{\|h\|}(B) \int_{I_{h}}\|f(s, y(s))-f(t, y(t))\| d s .
$$

Fixando $t$ e fazendo $h \rightarrow 0$, pela continuidade de $f(\cdot, y(\cdot))$ em $t$, temos

$$
\|f(s, y(s))-f(t, y(t))\| \leqslant \epsilon,
$$

para um arbitrário $\epsilon>0$. De (2.11) obtemos

$$
\left\|\frac{F(t+h)-F(t)}{h}-f(t, y(t))\right\| \leqslant \frac{1}{\|h\|}(B) \int_{I_{h}} \epsilon d s=\epsilon,
$$

o que implica que $F^{\prime}(t)=f(t, y(t))$. Logo, $y^{\prime}(t)=f(t, y(t))$. Portanto, $y$ é uma solução de Carathéodory de (2.1).

(d) Se $x$ é uma solução pseudo de (2.1), então $x$ é $A C$ e pseudo-diferenciável tal que $x\left(t_{0}\right)=x_{0}$ e para todo $x^{*} \in X^{*}$, temos $\left(x^{*} x\right)^{\prime}(t)=x^{*} f(t, x(t))$ em quase todo $t \in I$, com $x^{*} f(t, x(t))$ contínua em $I$. Logo, para todo $x^{*} \in X^{*}$,

$$
x^{*} x(t)-x^{*} x\left(t_{0}\right)=(L) \int_{t_{0}}^{t}\left(x^{*} x\right)^{\prime}(s) d s=(L) \int_{t_{0}}^{t} x^{*} f(s, x(s)) d s .
$$

Agora, da Definição 2.1.20, temos

$$
x(t)-x\left(t_{0}\right)=(P) \int_{t_{0}}^{t} f(s, x(s)) d s .
$$

Assim, $x$ é uma solução da equação integral (2.7). Reciprocamente, se $y$ é uma solução de (2.7), onde a integral considerada é a de Pettis, então

$$
y(t)=x_{0}+(P) \int_{t_{0}}^{t} f(s, y(s)) d s
$$

Logo, pela Definição 2.1.20, $x^{*} f(t, y(t))$ é $(L)$ integrável para todo $x^{*} \in X^{*}$. E ainda,

$$
x^{*} y(t)=x^{*} x_{0}+(L) \int_{t_{0}}^{t} x^{*} f(s, y(s)) d s, \quad \text { para todo } x^{*} \in X^{*} .
$$


Como $x^{*} f(\cdot, y(\cdot)): I \longrightarrow \mathbb{R}$ é $(L)$ integrável, então, pela Observação 4, existe uma função $h: I \longrightarrow \mathbb{R}, A C$, tal que $h\left(t_{0}\right)=x^{*} x_{0}$ e $h^{\prime}(t)=x^{*} f(t, y(t))$ em quase todo $t \in I$ e para todo $x^{*} \in X^{*}$. Logo por (2.12),

$$
x^{*} y(t)=x^{*} x_{0}+(L) \int_{t_{0}}^{t} h^{\prime}(s) d s=x^{*} x_{0}+h(t)-h\left(t_{0}\right)=x^{*} x_{0}+h(t)-x^{*} x_{0},
$$

o que implica que $x^{*} y(t)=h(t)$ para todo $x^{*} \in X^{*}$. Logo $x^{*} y: I \longrightarrow \mathbb{R}$ é $A C$ para todo $x^{*} \in X^{*}$ e, de maneira análoga a $(b)$, segue que $y$ é $A C$. E ainda, $x^{*} y\left(t_{0}\right)=$ $h\left(t_{0}\right)=x^{*} x_{0}$ para todo $x^{*} \in X^{*}, \operatorname{logo} x^{*}\left(y\left(t_{0}\right)-x_{0}\right)=0$ para todo $x^{*} \in X^{*}$. Usando o mesmo argumento de $(b)$, temos $y\left(t_{0}\right)=x_{0}$. Notemos também que $\left(x^{*} y\right)^{\prime}(t)=$ $h^{\prime}(t)=x^{*} f(t, x(t))$ em quase todo $t \in I$, assim $y$ é pseudo-diferenciável. Portanto, $y$ é uma solução pseudo de (2.1).

(e) Se $x$ é uma solução de Henstock de (2.1), então $x$ é uma função $A C G_{*}$. Logo $x$ é uma solução contínua de (2.1). Uma vez que $x^{\prime}(t)=f(t, x(t))$ em quase todo $t \in I$, então $x^{\prime}(t)$ é $(H)$ integrável pelo Teorema 2.1.14. E ainda,

$$
\text { (H) } \int_{t_{0}}^{t} f(s, x(s)) d s=(H) \int_{t_{0}}^{t} x^{\prime}(s) d s=x(t)-x\left(t_{0}\right)=x(t)-x_{0},
$$

o que implica que $x$ satisfaz (2.7). Reciprocamente, se $y$ é uma solução de (2.7), onde a integral considerada é a de Henstock, então

$$
y(t)=x_{0}+(H) \int_{t_{0}}^{t} f(s, y(s)) d s .
$$

Uma vez que $f(t, y(t))$ é $(H)$ integrável, então, pela Observação 2, existe uma função $A C G_{*}$, que denotaremos por $G$, tal que $G\left(t_{0}\right)=x_{0}$ e $G^{\prime}(t)=f(t, y(t))$ em quase todo $t$. De (2.13) temos

$$
y(t)=x_{0}+(H) \int_{t_{0}}^{t} G^{\prime}(s) d s=x_{0}+G(t)-G\left(t_{0}\right),
$$

o que implica que $y(t)=G(t)$, logo $y$ é $A C G_{*}$. E ainda, $y\left(t_{0}\right)=x_{0}$ e $y^{\prime}(t)=f(t, y(t))$ em quase todo $t$. Portanto, $y$ é uma solução de Henstock de (2.1).

(f) Para cada $x^{*} \in X^{*}$, podemos repetir o que fizemos em (e), produzindo assim uma solução de (2.7) fracamente $A C G_{*}$.

(g) É uma consequiência imediata da definição.

(h) Sabemos que uma função $f$ é $(D P)$ integrável se, e somente se, para cada $x^{*} \in X^{*}$ a função $x^{*} f$ é $(D)$ integrável, isto é, existe uma função $A C G$, dada por $F$, 
tal que $\left(x^{*} F\right)_{a p}^{\prime}=x^{*} f$ em quase todo $t$, e $x^{*} F$ é fracamente $A C G$ para cada $x^{*} \in X^{*}$. Daí, podemos repetir a prova de (e).

Observação 5: Como conseqüência do Teorema 2.1.27 e do diagrama em (2.6), afirmamos que é possível encontrarmos um problema de valor inicial da forma (2.1) em um espaço de Banach $X$ apropriado tal que se considerarmos os tipos de soluções para o problema (2.1) dados na Definição 2.1.26, teremos as seguintes inclusões: $(a) \subset(b) \subset(c) \subset(d) \subset(f) \subset(h),(c) \subset(e) \subset(f) \subset(h)$ e $(c) \subset(e) \subset(g) \subset(h) . \mathrm{E}$ ainda, todas estas inclusões são próprias.

Vimos então que existem vários tipos de soluções para o problema (2.1). Agora é natural nos perguntarmos quais são as condições que a função $f$ deve satisfazer para investigarmos a existência de soluções. Naturalmente, as condições sobre $f$ dependem do tipo de solução que desejamos obter. Em [5], Cichoń estabeleceu algumas condições sobre a função $f$ e assim obteve alguns resultados de existência de soluções, no sentido da Definição 2.1.26 para (2.1). Entretanto, na maior parte deste trabalho nos dedicaremos às soluções clássicas. Mas isso não nos impede de desenvolvermos, em algumas situações, resultados que envolvam outros tipos de soluções, como veremos, por exemplo, na Seção 2.7, onde obtemos um teorema de existência de soluções fracas em espaços de Banach reflexivos, utilizando as propriedades da função $f$ e destes espaços.

\subsection{Contra-Exemplos, Existência Local e Unici- dade}

A partir de agora consideraremos o problema (2.1), novamente em um espaço de Banach $X$, com $x_{0} \in X$. Contudo fixaremos um tipo de integral para a função $f(t, x(t))$, que será a integral de Riemann. Assim, uma solução para nós será a solução clássica, isto é, uma função $x: I \longrightarrow X$ contínua, continuamente diferenciável em ]$\left.t_{0}, t_{0}+a\right]$ e satisfazendo (2.1). Estamos exigindo apenas continuidade da função $x$, pois para nós, isto será suficiente. Na maioria das vezes, usaremos somente o termo "solução" para nos referirmos à "solução clássica". Somente nos referiremos 
ao termo "clássica" se estivermos interessados em estabelecer alguma relação com outros tipos de soluções. Nestas condições, uma das propriedades clássicas que deixa de ser válida quando $X$ tem dimensão infinita é o Teorema de Peano, isto é, que se $f$ é uma função contínua em uma vizinhança do ponto $\left(t_{0}, x_{0}\right)$, então existe pelo menos uma solução do problema de valor inicial (2.1) na vizinhança de $t_{0}$, com valores em $X$, assumindo o valor $x_{0}$ no instante $t_{0}$. O primeiro resultado nesta direção foi obtido por Dieudonné em [12], que construiu um contra-exemplo do Teorema de Peano em um espaço de Banach (Contra-Exemplo 1 a seguir). Yorke em [38], mostrou um outro contra-exemplo em um espaço de Hilbert (Contra-Exemplo 2 a seguir). Outra propriedade que deixa de ser válida é a extensão de soluções, também mostrado por Dieudonné em [12] (Contra-Exemplo 3 a seguir).

Contra-Exemplo 1: Consideremos $X$ um espaço de Banach contido em $l^{\infty}$ (espaço das seqüências limitadas de números reais ou complexos) dado por

$$
c_{0}=\left\{\left(x_{n}\right)_{n \in \mathbb{N}}: \quad x_{n} \in \mathbb{R}, \quad \lim _{n \rightarrow \infty} x_{n}=0\right\}
$$

com a norma

$$
\|x\|=\sup _{n}\left|x_{n}\right| .
$$

Para todo $x=\left(x_{n}\right)_{n \in \mathbb{N}} \in X=c_{0}$, tomemos $y$ como sendo a seqüência $\left(y_{n}\right)_{n \in \mathbb{N}}$ definida por

$$
y_{n}=\sqrt{\left|x_{n}\right|+\frac{1}{n+1}} .
$$

Daí, $y_{n} \in \mathbb{R}$ e $\lim _{n \rightarrow \infty} y_{n}=0$, logo $y=\left(y_{n}\right)_{n \in \mathbb{N}} \in c_{0}$.

Tomemos $y=f(x)$. Como a função $\sqrt{|x|}$ é uniformemente contínua em $\mathbb{R}$, então a aplicação $x \longmapsto f(x)$ é contínua do espaço $c_{0}$ nele mesmo. No entanto, mostraremos que a equação diferencial

$$
x^{\prime}=f(x)
$$

não admite nenhuma solução em $c_{0}$, assumindo o valor zero no instante $t=0$, isto é,

$$
x(0)=\odot, \quad \text { onde } \odot=(0,0, \ldots) \in c_{0} .
$$

De fato, suponhamos por contradição, que $x(t)$ seja uma solução do problema 
(2.14) e (2.15), então como (2.14) e (2.15) é equivalente a

$$
\begin{aligned}
& x_{n}^{\prime}=f\left(x_{n}\right) \\
& x_{n}(0)=0,
\end{aligned}
$$

para todo $n$, podemos escrever $x(t)=\left(x_{n}(t)\right)$, onde cada $n$-ésima coordenada $x_{n}$ é uma função derivável em uma vizinhança do ponto $t=0$ e satisfaz a equação diferencial ordinária uni-dimensional

$$
\begin{aligned}
& x_{n}^{\prime}(t)=\sqrt{\left|x_{n}(t)\right|+\frac{1}{n+1}} \\
& x_{n}(0)=0 .
\end{aligned}
$$

De (2.16) segue que $x_{n}(t)$ é estritamente crescente em $t$ e, uma vez que $x_{n}(0)=0$, então $x_{n}(t)>0$ para $0<t<\tau$, onde $\tau$ é suficientemente pequeno. Assim

$$
x_{n}^{\prime}(t)>\sqrt{x_{n}(t)}, \quad 0<t<\tau,
$$

ou ainda

$$
\frac{x_{n}^{\prime}(t)}{\sqrt{x_{n}(t)}}>1, \quad 0<t<\tau .
$$

Integrando ambos os lados de (2.17) de zero a $t$ obtemos

$$
x_{n}(t)>\frac{t^{2}}{4}, \quad 0<t<\tau, \quad \forall n .
$$

Logo $x_{n}$ está definida para todo $t \in \mathbb{R}$ e para $0 \leqslant t<\tau$ temos

$$
x_{n}(t) \geqslant \frac{t^{2}}{4}, \quad \forall n
$$

Notemos agora que não importa o quão pequeno escolhemos $\tau$, a seqüência $\left(x_{n}(t)\right)$ não converge para zero quando $n \rightarrow \infty$, o que contradiz o fato que $x(t)$ é uma solução de (2.14) e (2.15) e que, em particular, $x(t) \in c_{0}$.

Um argumento análogo vale para a esquerda de $t=0$. Portanto, embora a função $f$ seja contínua o problema (2.14) e (2.15) não possui solução em qualquer vizinhança contendo $t=0$.

Contra-Exemplo 2: Consideremos $H$ sendo um espaço de Hilbert dado por

$$
H=\left\{x=\left(x_{n}\right)_{n \geqslant 1}: \quad x_{n} \in \mathbb{R}, \quad\|x\|^{2}=\sum_{i=1}^{\infty} x_{i}^{2}<\infty\right\}=l^{2} .
$$


Sejam $P_{n}, n=0,1,2, \ldots$, as projeções dadas por

$$
\begin{aligned}
& P_{n}(x)=\left(0, \ldots, 0, x_{n+1}, x_{n+2}, \ldots\right), \quad n=1,2, \ldots \\
& P_{0}(x)=x .
\end{aligned}
$$

Para $t \in \mathbb{R}, x \in H$ e $\odot=(0,0, \ldots) \in H$ definimos

$$
P(t) x= \begin{cases}\odot, & \text { se } t \leqslant 0 \\ x, & \text { se } t \geqslant 1 \\ \left(2-2^{n} t\right) P_{n} x+\left(2^{n} t-1\right) P_{n-1} x, & \text { se } t \in\left[\frac{1}{2^{n}}, \frac{1}{2^{n-1}}\right], \quad n=1,2, \ldots\end{cases}
$$

Afirmamos que $P$ é contínua sobre $\mathbb{R} \times H$. De fato, $P$ é claramente contínua em $(t, x)$ se $t \neq 0$. Agora, para mostrarmos a continuidade nos pontos da forma $(0, p)$, onde $p=\left(p_{1}, p_{2}, \ldots\right)$, tomemos $t_{i} \rightarrow 0$ e $\left(x_{i}\right)$ sendo uma seqüência de pontos em $H$ com $x_{i} \rightarrow p$. Escrevemos $x_{i}=\left(x_{i 1}, x_{i 2}, \ldots\right)$. Para cada $n$ temos

$$
\begin{aligned}
& \left\|P\left(t_{i}\right) x_{i}\right\|^{2}=\left\|\left(2-2^{n} t_{i}\right) P_{n} x_{i}+\left(2^{n} t_{i}-1\right) P_{n-1} x_{i}\right\|^{2} \\
& =\left\|\left(2-2^{n} t_{i}\right)\left(0, \ldots, 0, x_{i, n+1}, x_{i, n+2}, \ldots\right)+\left(2^{n} t_{i}-1\right)\left(0, \ldots, 0, x_{i, n}, x_{i, n+1}, \ldots\right)\right\|^{2} \\
& =\| 2\left(0, \ldots, 0, x_{i, n+1}, x_{i, n+2}, \ldots\right)-\left(0, \ldots, 0, x_{i, n}, x_{i, n+1}, \ldots\right) \\
& +2^{n} t_{i}\left[\left(0, \ldots, 0, x_{i, n}, x_{i, n+1}, \ldots\right)-\left(0, \ldots, 0, x_{i, n+1}, x_{i, n+2}, \ldots\right)\right] \|^{2} \\
& =\left\|\left(0, \ldots, 0,-x_{i, n}, x_{i, n+1}, x_{i, n+2}, \ldots\right)+2^{n} t_{i}\left(0, \ldots, 0, x_{i, n}, 0, \ldots\right)\right\|^{2} .
\end{aligned}
$$

Assim, para cada $n$ com $t_{i} \leqslant \frac{1}{2^{n-1}}$, obtemos

$$
\left\|P\left(t_{i}\right) x_{i}\right\|^{2} \leqslant\left\|\left(0, \ldots, 0, x_{i, n}, x_{i, n+1}, x_{i, n+2}, \ldots\right)\right\|^{2}=\sum_{j=n}^{\infty} x_{i, j}^{2} .
$$

Portanto

$$
\left\|P\left(t_{i}\right) x_{i}\right\|^{2} \leqslant \sum_{j=n}^{\infty} x_{i, j}^{2}, \quad \text { para } t_{i} \leqslant \frac{1}{2^{n-1}}
$$

E ainda,

$$
\lim _{i \rightarrow \infty} \sup \left\|P\left(t_{i}\right) x_{i}\right\|^{2} \leqslant \lim _{i \rightarrow \infty} \sup \sum_{j=n}^{\infty} x_{i, j}^{2}=\sum_{j=n}^{\infty} p_{j}^{2} .
$$

Como isto vale para todo $n$, então, escolhendo $n$ grande,

$$
\lim _{i \rightarrow \infty} \sup \left\|P\left(t_{i}\right) x_{i}\right\|=0
$$


Portanto $P$ é contínua em $(0, p)$. Assim $P$ é contínua sobre $\mathbb{R} \times H$.

Consideremos agora

$$
G(x)=x\|x\|^{-\frac{1}{2}}, \quad \text { para } x \neq \odot \quad \text { e } \quad G(\odot)=\odot .
$$

Definimos

$$
A(x)=\left(\left|x_{1}\right|,\left|x_{2}\right|, \ldots\right) \quad \text { e } \quad v=\left(\frac{1}{2}, \frac{1}{2^{2}}, \frac{1}{2^{3}}, \ldots\right) .
$$

Tomamos a função

$$
F(t, x)=G(P(t) A(x))+P\left(\frac{t}{2}\right) v \max \left\{0, \frac{t^{2}}{4}-\|x\|\right\} .
$$

Como $P, A$ e $G$ são contínuas, então $F: \mathbb{R} \times H \longrightarrow H$ é contínua. Escrevemos $F=\left(F_{1}, F_{2}, \ldots\right)$. Mostraremos então que não existe solução $x(t)$ de

$$
x^{\prime}(t)=F(t, x(t))
$$

cujo domínio é um intervalo $J$ aberto contendo o ponto $t=0$ tal que $x(0)=\odot$.

Suponhamos, por contradição, que exista tal solução $x(t)$ de (2.18) continuamente diferenciável tal que $x(0)=\odot$, e escrevemos $x(t)=\left(x_{1}(t), x_{2}(t), \ldots\right)$. Uma vez que $P(t) x=\odot$ para $t \leqslant 0$, então $x^{\prime}(t)=\odot$ e $x(0)=\odot$, logo $x(t)=\odot$ para $t \leqslant 0$. Da definição de $A(x)$, segue que $F_{n}(t, x) \geqslant 0$ para todo $t, x$ e $n$. Logo $x_{n}^{\prime}(t)=F_{n}(t, x(t)) \geqslant 0$ e, assim, cada $x_{n}(t)$ é não decrescente. Como $x_{n}(0)=0$, então $x_{n}(t)>0$ para $t>0$, ou ainda, $x_{n}(t) \geqslant 0$ para $t \in J$. Assim

$$
A(x(t))=\left(\left|x_{1}(t)\right|,\left|x_{2}(t)\right|, \ldots\right)=\left(x_{1}(t), x_{2}(t), \ldots\right)=x(t) .
$$

E ainda,

$$
F(t, \odot)=G(P(t) \odot)+P\left(\frac{t}{2}\right) v \max \left\{0, \frac{t^{2}}{4}\right\}=\frac{t^{2}}{4} P\left(\frac{t}{2}\right) v \neq \odot
$$

para $t>0$. Então $x(t) \neq \odot$ para $t>0$.

Agora para $n=1,2, \ldots$ e $t \leqslant \frac{1}{2^{n}}, F_{n}(t, x)=0$ e $x_{n}^{\prime}(t)=0$. Então $x_{n}(t)=0$ para $t \leqslant \frac{1}{2^{n}}$. De fato, inicialmente temos $x^{\prime}=F(t, x)$, onde

$$
\begin{aligned}
F(t, x) & =G(P(t) x)+P\left(\frac{t}{2}\right) v \max \left\{0, \frac{t^{2}}{4}-\|x\|\right\} \\
& =\frac{P(t) x}{\|P(t) x\|^{\frac{1}{2}}}+P\left(\frac{t}{2}\right) v \max \left\{0, \frac{t^{2}}{4}-\|x\|\right\} .
\end{aligned}
$$


Para $t \in\left[\frac{1}{2^{n}}, \frac{1}{2^{n-1}}\right]$, com $n=1,2, \ldots$, temos

$$
\begin{aligned}
& P(t) v=\left(2-2^{n} t\right) P_{n} v+\left(2^{n} t-1\right) P_{n-1} v \\
& =\left(2-2^{n} t\right)\left(0, \ldots, 0, \frac{1}{2^{n+1}}, \frac{1}{2^{n+2}}, \ldots\right)+\left(2^{n} t-1\right)\left(0, \ldots, \frac{1}{2^{n}}, \frac{1}{2^{n+1}}, \frac{1}{2^{n+2}}, \ldots\right) \\
& =\left(0, \ldots, 0, \frac{\left(2^{n} t-1\right)}{2^{n}}, \frac{\left(2-2^{n} t\right)+\left(2^{n} t-1\right)}{2^{n+1}}, \frac{\left(2-2^{n} t\right)+\left(2^{n} t-1\right)}{2^{n+2}}, \ldots\right) .
\end{aligned}
$$

Logo

$$
P(t) v=(\underbrace{0, \ldots, 0}_{n-1 \text { primeiras }},\left(2^{n} t-1\right) v_{n}, v_{n+1}, v_{n+2}, \ldots), \quad \text { para } t \in\left[\frac{1}{2^{n}}, \frac{1}{2^{n-1}}\right] .
$$

Uma vez que para $t \in\left[\frac{1}{2^{n}}, \frac{1}{2^{n-1}}\right]$ temos $\frac{t}{2} \in\left[\frac{1}{2^{n+1}}, \frac{1}{2^{n}}\right]$, então

$$
P\left(\frac{t}{2}\right) v=(\underbrace{0, \ldots, 0}_{n \text { primeiras }},\left(2^{n+1} t-1\right) v_{n+1}, v_{n+2}, \ldots), \quad \text { para } t \in\left[\frac{1}{2^{n}}, \frac{1}{2^{n-1}}\right] .
$$

E ainda,

$$
\begin{aligned}
& P(t) x=\left(2-2^{n} t\right) P_{n} x+\left(2^{n} t-1\right) P_{n-1} x \\
& =\left(2-2^{n} t\right)\left(0, \ldots, 0, x_{n+1}, x_{n+2}, \ldots\right)+\left(2^{n} t-1\right)\left(0, \ldots, x_{n}, x_{n+1}, x_{n+2}, \ldots\right) \\
& =\left(0, \ldots,\left(2^{n} t-1\right) x_{n},\left(2-2^{n} t\right) x_{n+1}+\left(2^{n} t-1\right) x_{n+1},\left(2-2^{n} t\right) x_{n+2}+\left(2^{n} t-1\right) x_{n+2}, \ldots\right) .
\end{aligned}
$$

Logo

$$
P(t) x=(\underbrace{0, \ldots, 0}_{n-1 \text { primeiras }},\left(2^{n} t-1\right) x_{n}, x_{n+1}, x_{n+2}, \ldots), \quad \text { para } t \in\left[\frac{1}{2^{n}}, \frac{1}{2^{n-1}}\right] .
$$

Se $t \in[0,1]$, então existe um único $n \geqslant 1$ tal que $t \in\left[\frac{1}{2^{n}}, \frac{1}{2^{n-1}}\right]$. Logo $P(t) x$ é dada por (2.21). Agora se $t \leqslant \frac{1}{2^{n}}$, então existe um único $m \geqslant 1$ tal que $t \in\left[\frac{1}{2^{m}}, \frac{1}{2^{m-1}}\right]$, $\operatorname{com} \frac{1}{2^{m-1}} \leqslant \frac{1}{2^{n}}$. Logo

$$
2^{m-1} \geqslant 2^{n} \Longrightarrow m-1 \geqslant n \Longrightarrow m \geqslant n+1 \text {. }
$$

E ainda, para $t \in\left[\frac{1}{2^{m}}, \frac{1}{2^{m-1}}\right]$, segue de (2.21) que

$$
P(t) x=(\underbrace{0, \ldots, 0}_{m-1=n \text { primeiras }},\left(2^{m} t-1\right) x_{m}, x_{m+1}, x_{m+2}, \ldots), \quad \text { para } t \leqslant \frac{1}{2^{n}} .
$$


Como $\frac{t}{2} \in\left[\frac{1}{2^{m+1}}, \frac{1}{2^{m}}\right]$, segue de $(2.20)$ que

$$
P\left(\frac{t}{2}\right) v=(\underbrace{0, \ldots, 0}_{m=n+1 \text { primeiras }},\left(2^{m+1} t-1\right) v_{m+1}, v_{m+2}, \ldots), \quad \text { para } t \leqslant \frac{1}{2^{n}} .
$$

Portanto, para $n=1,2, \ldots$ e $t \leqslant \frac{1}{2^{n}}$ temos $F_{n}(t, x)=0$ e assim $x_{n}^{\prime}(t)=0$, com $x_{n}(0)=0$. Então $x_{n}(t)=0$ para $t \leqslant \frac{1}{2^{n}}$. Logo

$$
x(t)=\left(0, \ldots, 0, x_{n}, x_{n+1}, x_{n+2}, \ldots\right), \quad \text { para } t \in\left[\frac{1}{2^{n}}, \frac{1}{2^{n-1}}\right] .
$$

Uma vez que $F(t, x)$ é dada por (2.19), segue de (2.20) e (2.21) que para $t \in$ $\left[\frac{1}{2^{n}}, \frac{1}{2^{n-1}}\right]$,

$$
\begin{aligned}
F_{n}(t, x(t)) & =\frac{\left(2^{n} t-1\right) x_{n}(t)}{\|P(t) x\|^{\frac{1}{2}}}=\frac{\left(2^{n} t-1\right) x_{n}(t)}{\left[\left(2^{n} t-1\right)^{2} x_{n}^{2}(t)+x_{n+1}^{2}(t)+\ldots\right]^{\frac{1}{4}}} \\
& =\frac{\left(2^{n} t-1\right) x_{n}(t)}{\left[\left(\left(2^{n} t\right)^{2}-2^{n+1} t\right) x_{n}^{2}(t)+\sum_{k=n}^{\infty} x_{k}^{2}(t)\right]^{\frac{1}{4}}} .
\end{aligned}
$$

Assim, segue de (2.22) que para cada $n$,

$$
\begin{aligned}
& x_{n}^{\prime}(t)=\frac{\left(2^{n} t-1\right) x_{n}(t)}{\left[\left(2^{n} t-2\right) 2^{n} t x_{n}^{2}(t)+\|x(t)\|^{2}\right]^{\frac{1}{4}}}=f_{n}\left(t, x_{n}(t)\right), \quad \text { para } t \in\left[\frac{1}{2^{n}}, \frac{1}{2^{n-1}}\right] \\
& x_{n}\left(\frac{1}{2^{n}}\right)=0 .
\end{aligned}
$$

Como $x(t) \neq \odot$ para $t>0$ e por hipótese $x(t)$ é uma solução de (2.18), então claramente a função $f_{n}\left(t, x_{n}(t)\right)$ é contínua e também podemos mostrar que $f_{n}$ é de classe $C^{1}$ na variável $x_{n}$. Logo $f_{n}$ é localmente Lipschitziana. Assim, pelo Teorema de Picard em dimensão finita, temos a existência e unicidade de soluções para o problema (2.23). Portanto sobre $\left[\frac{1}{2^{n}}, \frac{1}{2^{n-1}}\right]$ a coordenada $x_{n}$ deve ser zero, uma vez que $x_{n}(t)=0$ é uma solução de (2.23), e ainda, para todo $t$ segue de (2.21) que

$$
\begin{aligned}
P(t) A(x(t)) & =P(t) x(t)=\left(0, \ldots, 0,\left(2^{n} t-1\right) x_{n}(t), x_{n+1}(t), \ldots\right) \\
& =\left(0, \ldots, 0,0, x_{n+1}(t), \ldots\right)=x(t) .
\end{aligned}
$$

Considerando agora $\rho(t)=\|x(t)\|^{2}$ e tomando o produto interno $\langle x, y\rangle=\sum_{i=1}^{\infty} x_{i} y_{i}$, temos

$$
\begin{aligned}
\frac{d \rho(t)}{d t} & =2\left\langle x(t), x^{\prime}(t)\right\rangle \\
& =2\left\langle x(t), G(x(t))+P\left(\frac{t}{2}\right) v \max \left\{0, \frac{t^{2}}{4}-\|x(t)\|\right\}\right\rangle \\
& =2\|x(t)\|^{-\frac{1}{2}}\langle x(t), x(t)\rangle+2\left\langle x(t), P\left(\frac{t}{2}\right) v\right\rangle \max \left\{0, \frac{t^{2}}{4}-\|x(t)\|\right\} \\
& =2\|x(t)\|^{\frac{3}{2}}+2 \max \left\{0, \frac{t^{2}}{4}-\|x(t)\|\right\} \sum_{i=1}^{\infty} x_{i}\left(P\left(\frac{t}{2}\right) v\right)_{i} .
\end{aligned}
$$


Já vimos que $x_{i}(t) \geqslant 0$ para todo $t \in J, v_{i}=\frac{1}{2^{i}}>0$ para todo $i=1,2, \ldots$ e para $\frac{1}{2^{i}} \leqslant t \leqslant \frac{1}{2^{i-1}}$ temos $1 \leqslant 2^{i+1} t-1 \leqslant 3$. Logo por $(2.20),\left(P\left(\frac{t}{2}\right) v\right)_{i} \geqslant 0$ para $i=$ $1,2, \ldots$. Conseqüentemente $\sum_{i=1}^{\infty} x_{i}\left(P\left(\frac{t}{2}\right) v\right)_{i} \geqslant 0$ e ainda $\max \left\{0, \frac{t^{2}}{4}-\|x(t)\|\right\} \geqslant 0$. Assim

$$
\frac{d \rho(t)}{d t} \geqslant 2\|x(t)\|^{\frac{3}{2}}=2 \rho^{\frac{3}{4}}(t), \quad t>0, \quad t \in J
$$

A princípio, a desigualdade em (2.24) vale para $0 \leqslant t \leqslant 1$, mas para $t>1$ obtemos $P(t) x(t)=x(t)$ e $P\left(\frac{t}{2}\right) v=v$ e o resultado é análogo. Assim, resolvendo (2.24), temos

$$
\rho(t) \geqslant\left(\frac{t}{2}\right)^{4}, \quad \text { para } t>0
$$

Logo $\|x(t)\| \geqslant \frac{t^{2}}{4}$ para $t \geqslant 0$ e, assim, $\max \left\{0, \frac{t^{2}}{4}-\|x(t)\|\right\}=0$.

Portanto, segue de (2.19) que nossa solução particular $x(t)$ satisfaz, para $t \in J$,

$$
x^{\prime}(t)=x(t)\|x(t)\|^{-\frac{1}{2}}, \quad t>0 .
$$

Isto é,

$$
\begin{aligned}
& x_{n}^{\prime}(t)=x_{n}(t) \rho(t)^{-\frac{1}{4}}, \quad \text { para } n=1,2, \ldots \text { e } t>0 \\
& x_{n}\left(\frac{1}{2^{n}}\right)=0 .
\end{aligned}
$$

Novamente pelo Teorema de Picard em dimensão finita, a solução de (2.25) para cada $n$ (para qualquer função escalar contínua $\rho(t)>0$ ) é $x_{n}(t)=0$, contradizendo nosso resultado anterior que $x(t) \neq \odot$ para $t>0$. Assim não existe uma solução $x(t)$ de (2.18) satisfazendo $x(0)=\odot$.

Observação: O Contra-Exemplo 1 depende fortemente das propriedades de $c_{0}$, que não é reflexivo. Entretanto o Contra-Exemplo 2 é dado em um espaço de Hilbert, e estes são sempre reflexivos (ver [26], pág. 242, Teorema 4.6-6). Apesar de não conseguirmos garantir a existência de soluções clássicas em espaços reflexivos, sob certas condições, conseguimos garantir a existência de soluções fracas. Na Seção 2.7, veremos com mais detalhes esses comentários.

Vimos então que a continuidade da função $f$ em $t$ não é suficiente para garantir existência de soluções para o problema (2.1), entretanto acrescentanto a hipótese 
que a função $f$ é localmente Lipschitziana em $x$, podemos provar a existência local de soluções e até mesmo a unicidade de soluções para o problema (2.1), de maneira análoga ao caso em que $X=\mathbb{R}^{n}$. Contudo, existem outras maneiras de solucionarmos este problema de existência, por exemplo, trabalhando com condições de compacidade, como veremos na Seção 2.6, mas no momento nos restringiremos àquela somente.

Teorema 2.2.1 (Existência Local e Unicidade) Consideremos o "cilindro"

$$
R_{0}=\left\{(t, x) \in \mathbb{R} \times X: \quad t_{0} \leqslant t \leqslant t_{0}+a, \quad\left\|x-x_{0}\right\| \leqslant \beta\right\},
$$

onde a e $\beta$ são constantes positivas. Seja $f: R_{0} \rightarrow X$ contínua em $t$ para cada $x$ fixado. Suponhamos que $\|f(t, x)\| \leqslant M$ para $(t, x) \in R_{0}$ e que $f$ seja Lipschitziana em $x$, isto é, $\left\|f\left(t, x_{1}\right)-f\left(t, x_{2}\right)\right\| \leqslant K\left\|x_{1}-x_{2}\right\|$ para $\left(t, x_{1}\right),\left(t, x_{2}\right) \in R_{0}, \operatorname{com} K e$ $M$ constantes não negativas. Então existe uma única solução $x(t)$ para o problema de valor inicial (2.1) sobre $\left[t_{0}, t_{0}+\alpha\right]$, onde $\alpha=\min \left\{a, \frac{\beta}{M}\right\}$.

Demonstração: Na demonstração deste teorema, utilizaremos o Teorema do Ponto Fixo de Banach ou da Contração (ver [26], pág. 300, Teorema 5.1-2). Consideremos incialmente $C_{\alpha}=C\left(\left[t_{0}, t_{0}+\alpha\right], X\right)$, sendo o espaço de Banach das funções contínuas $x:\left[t_{0}, t_{0}+\alpha\right] \longrightarrow X$, com a norma

$$
\|x\|_{\infty}=\sup _{t \in\left[t_{0}, t_{0}+\alpha\right]}\|x(t)\|
$$

Consideremos neste espaço a bola fechada

$$
\bar{B}_{\beta}=\bar{B}_{\beta}\left(x_{0}\right)=\left\{x \in C_{\alpha}:\left\|x-x_{0}\right\|_{\infty} \leqslant \beta\right\} . \quad \text { (é completo) }
$$

Tomemos o operador

$$
(T x)(t)=x_{0}+\int_{t_{0}}^{t} f(s, x(s)) d s
$$

Notemos que $T x$ é um operador contínuo. E ainda o operador $T$ leva a bola fechada $\bar{B}_{\beta}$ nela mesma, isto é, $T \bar{B}_{\beta} \subset \bar{B}_{\beta}$, uma vez que

$$
\left\|(T x)(t)-x_{0}\right\|=\left\|\int_{t_{0}}^{t} f(s, x(s)) d s\right\| \leqslant M\left|t-t_{0}\right| \leqslant M \alpha \leqslant M \frac{\beta}{M}=\beta .
$$


Logo

$$
\sup _{t \in\left[t_{0}, t_{0}+\alpha\right]}\left\|(T x)(t)-x_{0}\right\| \leqslant \beta \Longrightarrow\left\|T x-x_{0}\right\|_{\infty} \leqslant \beta .
$$

Mostremos agora que o operador $T^{n}$ é uma contração em $\bar{B}_{\beta}$ para $n$ suficientemente grande. Tomando $x_{1}, x_{2} \in \bar{B}_{\beta}$, temos

$$
\begin{aligned}
& \left\|\left(T x_{2}\right)(t)-\left(T x_{1}\right)(t)\right\|=\left\|\int_{t_{0}}^{t} f\left(s, x_{2}(s)\right) d s-\int_{t_{0}}^{t} f\left(s, x_{1}(s)\right) d s\right\| \\
& \leqslant \int_{t_{0}}^{t} K\left\|x_{2}(s)-x_{1}(s)\right\| d s \leqslant \int_{t_{0}}^{t} K\left\|x_{2}-x_{1}\right\|_{\infty} d s \leqslant K\left|t-t_{0}\right|\left\|x_{2}-x_{1}\right\|_{\infty} .
\end{aligned}
$$

Provaremos, por indução sobre $n$, que

$$
\left\|\left(T^{n} x_{2}\right)(t)-\left(T^{n} x_{1}\right)(t)\right\| \leqslant \frac{K^{n}\left|t-t_{0}\right|^{n}}{n !}\left\|x_{2}-x_{1}\right\|_{\infty}
$$

Já vimos que (2.26) é verdadeiro para $n=1$. Suponhamos verdadeiro para $n=r$ e mostremos que é verdadeiro para $n=r+1$. Tomando $x_{1}, x_{2} \in \bar{B}_{\beta}$, temos

$$
\begin{aligned}
& \left\|\left(T^{r+1} x_{2}\right)(t)-\left(T^{r+1} x_{1}\right)(t)\right\|=\left\|\int_{t_{0}}^{t} f\left(s,\left(T^{r} x_{2}\right)(s)\right) d s-\int_{t_{0}}^{t} f\left(s,\left(T^{r} x_{1}\right)(s)\right) d s\right\| \\
& \leqslant \int_{t_{0}}^{t} K\left\|\left(T^{r} x_{2}\right)(s)-\left(T^{r} x_{1}\right)(s)\right\| d s \leqslant \int_{t_{0}}^{t} K K^{r} \frac{\left|s-t_{0}\right|^{r}}{r !}\left\|x_{2}-x_{1}\right\|_{\infty} d s \\
& =K^{r+1} \frac{\left|t-t_{0}\right|^{r+1}}{(r+1) !}\left\|x_{2}-x_{1}\right\|_{\infty} .
\end{aligned}
$$

Portanto (2.26) é verdadeiro para $n=r+1$. Logo, aplicando o $\sup _{t \in\left[t_{0}, t_{0}+\alpha\right]}$ em (2.26), temos

$$
\left\|T^{n} x_{2}-T^{n} x_{1}\right\|_{\infty} \leqslant \frac{(K \alpha)^{n}}{n !}\left\|x_{2}-x_{1}\right\|_{\infty}
$$

Quando $n \rightarrow \infty$ segue que $\frac{(K \alpha)^{n}}{n !} \rightarrow 0$. Logo existe $n_{0} \geqslant 1$ tal que $\frac{(K \alpha)^{n}}{n_{0} !}<1$. Assim, o operador $T^{n}$ é uma contração em $\bar{B}_{\beta}$ para $n \rightarrow \infty$. Portanto, pelo Teorema do Ponto Fixo de Banach, $T^{n}$ possui um único ponto fixo $x(t) \in \bar{B}_{\beta}$. Conseqüentemente $x(t)$ também é o único ponto fixo de $T$, e este é exatamente a solução de (2.1), pois

$$
(T x)(t)=x(t) \Longrightarrow x(t)=x_{0}+\int_{t_{0}}^{t} f(s, x(s)) d s,
$$

daí

$$
x\left(t_{0}\right)=x_{0} \quad \text { e } \quad \frac{d x}{d t}=f(t, x(t)) .
$$


Exemplo: Seja $X$ uma álgebra de Banach sobre um corpo $\mathbb{K}$ com unidade $u$, isto é, $X$ é um espaço de Banach tal que, para cada par ordenado de elementos $x$, $y \in X$, um único produto $x y \in X$ é definido de tal forma que para todo $x, y, z \in X$ e $\alpha \in \mathbb{K}$, temos:

(i) $(x y) z=x(y z)$

(ii) $x(y+z)=x y+x z$

(iii) $(x+y) z=x z+y z$

(iv) $\alpha(x y)=(\alpha x) y=x(\alpha y)$;

(v) $\|x y\| \leqslant\|x\|\|y\|$;

(vi) $\|u\|=1$.

A função $f(t, x)=c x$, onde $c, x \in X$ e $c$ é fixado, satisfaz as hipóteses do Teorema 2.2.1, com $t_{0}=0$ e $x_{0}=u$. De fato, $f$ é claramente contínua e ainda $f$ é Lipschitziana e limitada, pois

$$
\begin{gathered}
\left\|f\left(t, x_{1}\right)-f\left(t, x_{2}\right)\right\|=\left\|c\left(x_{1}-x_{2}\right)\right\| \leqslant\|c\|\left\|x_{1}-x_{2}\right\| . \\
\|f(t, x)\|=\|c x\| \leqslant\|c\|\|x\| \leqslant(\beta+1)\|c\| .
\end{gathered}
$$

Portanto o problema de Cauchy

$$
\begin{aligned}
& \frac{d x}{d t}=c x \\
& x(0)=u,
\end{aligned}
$$

tem uma única solução e esta solução é dada por

$$
x(t)=u+\sum_{n=1}^{\infty} \frac{t^{n} c^{n}}{n !}=e^{t c}, \quad \text { em } X .
$$

Vimos que o Teorema 2.2.1 é um resultado local, no caso em que $X=\mathbb{R}^{n}$, $I=\left[t_{0}, t_{0}+a\right]$ e a função $f \in C(I \times X, X)$ é localmente Lipschitziana em $x$, sabemos que para cada $x_{0} \in X$ existe um intervalo maximal $J \subset I$, por exemplo, $J=\left[t_{0}, \delta[\right.$, com $0<\delta \leqslant t_{0}+a$, em que existe uma única solução $x(t)$ de (2.1). Se $J \neq I$ então

$$
\|x(t)\| \rightarrow \infty, \quad \text { quando } t \rightarrow \delta
$$

Isto também vale quando $f$ não é localmente Lipschitziana, no entanto temos outras hipóteses que asseguram que o problema (2.1) possui uma única solução para todo $\left(t_{0}, x_{0}\right)$. Se $f \in C\left(\left[t_{0}-a, t_{0}\right] \times X, X\right)$, um resultado similar vale para a esquerda 
de $t_{0}$. Estes resultados não valem se $X$ é um espaço de Banach de dimensão infinita, como veremos a seguir no Contra-Exemplo 3.

Contra-Exemplo 3: Consideremos agora o problema (2.1) sobre o mesmo espaço de Banach $X=c_{0}$ do Contra-Exemplo 1. Tomemos em $c_{0}$ a sequiência $e_{n}$ em que todos os termos são iguais a zero, exceto o termo de índice $n$, que é igual a um, isto é, $e_{n}=(0, \ldots, 0,1,0, \ldots)$.

Consideremos a aplicação $[\cdot]^{+}: \mathbb{R} \longrightarrow \mathbb{R}^{+}$dada por

$$
[x]^{+}= \begin{cases}0, & \text { se } x \leqslant 0 \\ x, & \text { se } x>0 .\end{cases}
$$

Tomemos, para todo $x=\left(x_{n}\right) \in c_{0}$, a seqüência de funções $f_{n}: c_{0} \longrightarrow c_{0}$ dada por

$$
f_{n}(x)=\left[2\left(x_{n}+x_{n+1}\right)-1\right]^{+}\left(e_{n+1}-e_{n}\right) .
$$

Ou ainda,

$$
f_{n}(x)=(0, \ldots, 0, \underbrace{-\left[2\left(x_{n}+x_{n+1}\right)-1\right]^{+}}_{n \text {-ésima }}, \underbrace{\left[2\left(x_{n}+x_{n+1}\right)-1\right]^{+}}_{n+1 \text {-ésima }}, 0, \ldots) .
$$

Estudaremos algumas das propriedades da função $f_{n}$. Inicialmente, afirmamos que $f_{n}$ é contínua em $c_{0}$. De fato, tomemos $\left(x^{m}\right)_{m \in \mathbb{N}}$ tal que $x^{m} \rightarrow x$ em $c_{0}$, quando $m \rightarrow \infty$, mostremos que $f_{n}\left(x^{m}\right) \rightarrow f_{n}(x)$ em $c_{0}$, quando $m \rightarrow \infty$,

$$
\begin{aligned}
& \left\|f_{n}\left(x^{m}\right)-f_{n}(x)\right\|=\|\left(0, \ldots, 0,-\left[2\left(x_{n}^{m}+x_{n+1}^{m}\right)-1\right]^{+}+\left[2\left(x_{n}+x_{n+1}\right)-1\right]^{+}\right. \\
& \left.\left[2\left(x_{n}^{m}+x_{n+1}^{m}\right)-1\right]^{+}-\left[2\left(x_{n}+x_{n+1}\right)-1\right]^{+}, 0, \ldots\right) \|
\end{aligned}
$$

Como $x^{m} \rightarrow x$ quando $m \rightarrow \infty$, não podemos ter $2\left(x_{n}^{m}+x_{n+1}^{m}\right)-1>0 \mathrm{e}$ $2\left(x_{n}+x_{n+1}\right)-1<0$ ou $2\left(x_{n}^{m}+x_{n+1}^{m}\right)-1<0$ e $2\left(x_{n}+x_{n+1}\right)-1>0$. Então, temos apenas dois casos a considerar:

$\underline{1^{\circ} \text { caso: }}$ se $2\left(x_{n}^{m}+x_{n+1}^{m}\right)-1>0$ e $2\left(x_{n}+x_{n+1}\right)-1>0$, então

$$
\begin{aligned}
& \left\|f_{n}\left(x^{m}\right)-f_{n}(x)\right\| \\
= & \left\|\left(0, \ldots, 0,-2\left(x_{n}^{m}+x_{n+1}^{m}\right)+2\left(x_{n}+x_{n+1}\right), 2\left(x_{n}^{m}+x_{n+1}^{m}\right)-2\left(x_{n}+x_{n+1}\right), 0, \ldots\right)\right\| \\
= & \left\|\left(0, \ldots, 0,2\left[\left(x_{n}-x_{n}^{m}\right)+\left(x_{n+1}-x_{n+1}^{m}\right)\right], 2\left[\left(x_{n}^{m}-x_{n}\right)+\left(x_{n+1}^{m}-x_{n+1}\right)\right], 0, \ldots\right)\right\| .
\end{aligned}
$$


Assim, quando $m \rightarrow \infty$, temos $\left\|f_{n}\left(x^{m}\right)-f_{n}(x)\right\| \rightarrow 0$.

2 ${ }^{\circ}$ caso: se $2\left(x_{n}^{m}+x_{n+1}^{m}\right)-1 \leqslant 0$ e $2\left(x_{n}+x_{n+1}\right)-1 \leqslant 0$, então $\left\|f_{n}\left(x^{m}\right)-f_{n}(x)\right\|=0$, e o resultado é imediato.

Mostremos agora que $f_{n}$ é Lipschitziana. Tomando $x, y \in c_{0}$ temos

$$
\begin{aligned}
& \left\|f_{n}(x)-f_{n}(y)\right\|=\|\left(0, \ldots, 0,-\left[2\left(x_{n}+x_{n+1}\right)-1\right]^{+},\left[2\left(x_{n}+x_{n+1}\right)-1\right]^{+}, 0, \ldots\right) \\
& -\left(0, \ldots, 0,-\left[2\left(y_{n}+y_{n+1}\right)-1\right]^{+},\left[2\left(y_{n}+y_{n+1}\right)-1\right]^{+}, 0, \ldots\right) \| \\
& =\|\left(0, \ldots, 0,-\left[2\left(x_{n}+x_{n+1}\right)-1\right]^{+}+\left[2\left(y_{n}+y_{n+1}\right)-1\right]^{+}\right. \\
& \left.\left[2\left(x_{n}+x_{n+1}\right)-1\right]^{+}-\left[2\left(y_{n}+y_{n+1}\right)-1\right]^{+}, 0, \ldots\right) \| .
\end{aligned}
$$

Agora temos três casos a considerar:

$\underline{1^{\circ} \text { caso: }}$ se $2\left(x_{n}+x_{n+1}\right)-1>0$ e $2\left(y_{n}+y_{n+1}\right)-1>0$, então

$$
\begin{aligned}
& \left\|f_{n}(x)-f_{n}(y)\right\| \\
= & \left\|\left(0, \ldots, 0,2\left(y_{n}-x_{n}\right)+2\left(y_{n+1}-x_{n+1}\right), 2\left(x_{n}-y_{n}\right)+2\left(x_{n+1}-y_{n+1}\right), 0, \ldots\right)\right\| \\
= & \sup _{n}\left\{\left|2\left(y_{n}-x_{n}\right)+2\left(y_{n+1}-x_{n+1}\right)\right|\right\} \leqslant \sup _{n}\left\{2\left|y_{n}-x_{n}\right|+2\left|y_{n+1}-x_{n+1}\right|\right\} \\
\leqslant & 2 \sup _{n}\left\{\left|y_{n}-x_{n}\right|\right\}+2 \sup _{n}\left\{\left|y_{n+1}-x_{n+1}\right|\right\}=2\|x-y\|+2\|x-y\|,
\end{aligned}
$$

$\log 0$

$$
\left\|f_{n}(x)-f_{n}(y)\right\| \leqslant 4\|x-y\| .
$$

20 caso: se $2\left(x_{n}+x_{n+1}\right)-1>0$ e $2\left(y_{n}+y_{n+1}\right)-1 \leqslant 0$, então efetuamos cálculos semelhantes ao caso anterior e obtemos

$$
\left\|f_{n}(x)-f_{n}(y)\right\| \leqslant 4\|x-y\|
$$

3 $3^{\circ}$ caso: se $2\left(x_{n}+x_{n+1}\right)-1 \leqslant 0$ e $2\left(y_{n}+y_{n+1}\right)-1 \leqslant 0$, então o resultado é imediato.

A função $f_{n}$ também satisfaz a seguinte propriedade: para $\|x\| \leqslant \frac{1}{4} \operatorname{temos} f_{n}(x)=$ $\odot$. Uma vez que $\|x\| \leqslant \frac{1}{4}$, então $\left|x_{n}\right| \leqslant \frac{1}{4}$ para todo $n$. Assim

$$
\left|x_{n}+x_{n+1}\right| \leqslant\left|x_{n}\right|+\left|x_{n+1}\right| \leqslant \frac{1}{2} \Longrightarrow 2\left|x_{n}+x_{n+1}\right| \leqslant 1 \Longrightarrow 2\left|x_{n}+x_{n+1}\right|-1 \leqslant 0 .
$$

Logo $\left[2\left(x_{n}+x_{n+1}\right)-1\right]^{+}=0$ e, conseqüentemente, $f_{n}(x)=\odot$. 
Para $x=\lambda e_{n}+(1-\lambda) e_{n+1}$, com $\lambda$ um escalar qualquer, temos $f_{n}(x)=e_{n+1}-e_{n}$. De fato,

$f_{n}\left(\lambda e_{n}+(1-\lambda) e_{n+1}\right)=f_{n}(0, \ldots, \underbrace{\lambda}_{n \text {-ésima }}, \underbrace{1-\lambda}_{n+1 \text {-́́sima }}, 0, \ldots)=(0, \ldots, 0,-1,1,0, \ldots)=e_{n+1}-e_{n}$.

Por outro lado, para todo inteiro $n>0$, seja $\varphi_{n}$ uma função numérica satisfazendo $\varphi_{n} \geqslant 0$, definida e contínua no intervalo $\frac{1}{n+1} \leqslant t \leqslant \frac{1}{n}$, com $\varphi_{n}\left(\frac{1}{n+1}\right)=\varphi_{n}\left(\frac{1}{n}\right)=0$ e tal que

$$
\int_{\frac{1}{n+1}}^{\frac{1}{n}} \varphi_{n}(t) d t=1
$$

Seja $I=]-\infty, 1]$ e definimos $f: I \times c_{0} \longrightarrow c_{0}$ por

$$
f(t, x)= \begin{cases}\varphi_{n}(t) f_{n}(x), & \text { se } \frac{1}{n+1} \leqslant t \leqslant \frac{1}{n} \text { e } x \in c_{0} \\ \odot, & \text { se } t \leqslant 0 \text { e } x \in c_{0} .\end{cases}
$$

A função $f$ é contínua em todos os pontos $(t, x)$ de $I \times c_{0}$ tal que $t \neq 0$. E ainda é localmente Lipschitziana na variável $x$, isto é imediato se $t \leqslant 0$. Agora se $\frac{1}{n+1} \leqslant t \leqslant \frac{1}{n}$, segue de $(2.27)$ que

$$
\|f(t, x)-f(t, y)\| \leqslant\left|\varphi_{n}(t)\right|\left\|f_{n}(x)-f_{n}(y)\right\| .
$$

Como $\varphi_{n}$ é contínua em $\frac{1}{n+1} \leqslant t \leqslant \frac{1}{n}$, então é localmente limitada e ainda $f_{n}$ é Lipschitziana. Logo

$$
\|f(t, x)-f(t, y)\| \leqslant M\|x-y\|,
$$

com $M \geqslant 0$, ou seja, $f$ é localmente Lipschitziana.

E ainda, $f$ também é contínua em todo ponto da forma $(0, p)$, onde $p=\left(p_{n}\right) \in c_{0}$. De fato, uma vez que $\lim _{n \rightarrow \infty} p_{n}=0$, então existe $m \in \mathbb{N}$ tal que para $n \geqslant m$ temos $\left|p_{n}\right| \leqslant \frac{1}{8}$. E ainda para todo $x=\left(x_{n}\right)$ satisfazendo $\|x-p\| \leqslant \frac{1}{8}$ com $n \geqslant m$, temos $\left|x_{n}\right| \leqslant \frac{1}{4}$, uma vez que

$$
\left|x_{n}-p_{n}\right| \leqslant\|x-p\| \leqslant \frac{1}{8} \Longrightarrow\left|x_{n}\right| \leqslant \frac{1}{8}+\left|p_{n}\right| \Longrightarrow\left|x_{n}\right| \leqslant \frac{1}{4}, \quad \forall n \geqslant m .
$$

Com base no que vimos anteriormente, segue que $f_{n}(x)=\odot$ para todo $n \geqslant m$. E assim, $f(t, x)=\odot$ para $0 \leqslant t \leqslant \frac{1}{m}$ e $\|x-p\| \leqslant \frac{1}{8}$.

Definimos, agora, uma seqüência de funções $v_{n}:[0,1] \longrightarrow c_{0}$ da seguinte maneira

$$
v_{1}(t)= \begin{cases}\odot, & \text { para } t<\frac{1}{2} \\ e_{1}+\left(e_{2}-e_{1}\right) \int_{t}^{1} \varphi_{1}(s) d s, & \text { para } \frac{1}{2} \leqslant t \leqslant 1\end{cases}
$$




$$
v_{n}(t)= \begin{cases}\odot, & \text { para } t>\frac{1}{n} \text { ou } t<\frac{1}{n+1} \\ e_{n}+\left(e_{n+1}-e_{n}\right) \int_{t}^{\frac{1}{n}} \varphi_{n}(s) d s, & \text { para } \frac{1}{n+1} \leqslant t \leqslant \frac{1}{n} .\end{cases}
$$

Consideremos a função $x:] 0,1] \longrightarrow c_{0}$ dada por

$$
x(t)=\sum_{n=1}^{\infty} v_{n}(t) .
$$

Notemos que se $t=1$, então $v_{1}(1)=e_{1}$, e como $1>\frac{1}{n}$ para $n \geqslant 2$, segue que $v_{n}(1)=\odot$ para todo $n \geqslant 2$. Agora se $t \neq 1$, então existe um único $n$ tal que $t \in\left[\frac{1}{n+1}, \frac{1}{n}\right]$. Logo $v_{i}(t)=\odot$ para $i \neq n \mathrm{e}$

$$
v_{n}(t)=e_{n}+\left(e_{n+1}-e_{n}\right) \int_{t}^{\frac{1}{n}} \varphi_{n}(s) d s .
$$

Daí, a soma dada em (2.28) comporta apenas um número finito de termos não nulos. Assim, para $\frac{1}{n+1} \leqslant t \leqslant \frac{1}{n}$ temos

$x(t)=e_{n}+\left(e_{n+1}-e_{n}\right) \int_{t}^{\frac{1}{n}} \varphi_{n}(s) d s=\left(0, \ldots, 0,1-\int_{t}^{\frac{1}{n}} \varphi_{n}(s) d s, \int_{t}^{\frac{1}{n}} \varphi_{n}(s) d s, 0, \ldots\right)$.

Então para $0<t \leqslant 1$, temos

$$
\|x(t)\|=\left\|\left(0, \ldots, 0,1-\int_{t}^{\frac{1}{n}} \varphi_{n}(s) d s, \int_{t}^{\frac{1}{n}} \varphi_{n}(s) d s, 0, \ldots\right)\right\| .
$$

Portanto

$$
\|x(t)\| \leqslant 1, \quad \text { para } 0<t \leqslant 1 .
$$

Notemos que

$$
f_{n}(x(t))=\left[2\left(1-\int_{t}^{\frac{1}{n}} \varphi_{n}(s) d s+\int_{t}^{\frac{1}{n}} \varphi_{n}(s) d s\right)-1\right]^{+}\left(e_{n+1}-e_{n}\right)=\left(e_{n+1}-e_{n}\right) .
$$

Logo, derivando $x(t)$ com relação a $t$, obtemos

$$
x^{\prime}(t)=-\left(e_{n+1}-e_{n}\right) \varphi_{n}(t)=-\varphi_{n}(t) f_{n}(x(t))=-f(t, x(t)) .
$$

Assim $x(t)$ satisfaz o problema de valor inicial

$$
\begin{aligned}
& x^{\prime}(t)=-f(t, x(t)), \quad 0<t \leqslant 1 \\
& x\left(\frac{1}{n}\right)=e_{n},
\end{aligned}
$$


com $-f$ contínua na variável $t$ e localmente Lipschitziana na variável $x$.

Uma vez que $x\left(\frac{1}{n}\right)=e_{n}$ e $\left(e_{n}\right)$ não tem um limite quando $n \rightarrow \infty$, então $x(t)$ não tende a um limite quando $t \rightarrow 0$, o que mostra que $J=10,1]$ é o maior intervalo em que existe uma única solução de (2.29) dada por (2.28), que assume o valor $e_{1}$ no ponto $t=1$. Entretanto, $J \neq I$ e $\|x(t)\| \leqslant 1$ para $t \in J$.

\subsubsection{Comentários sobre o Teorema de Peano em Dimensão Infinita}

O motivo pelo qual a prova clássica do Teorema de Peano falha em um espaço de Banach de dimensão infinita é que a bola fechada unitária não é compacta e a prova do Teorema de Peano em dimensão finita depende essencialmente de um argumento de compacidade, o Teorema de Ascoli-Arzelá, cuja demonstração pode ser encontrada em [31], pág. 149, Teorema 3.17.24.

Teorema 2.2.2 (Ascoli-Arzelá) Sejam $X$ um espaço de Banach e $C(J, X)$ o espaço de todas as funções contínuas $x: J \longrightarrow X$, com a norma $\|x\|_{\infty}=\sup _{t \in J}\|x(t)\|$. Um subconjunto $S \subset C(J, X)$ é relativamente compacto se, e somente se, $S$ é eqüicontínuo $e S(t)=\{x(t): x \in S\}$ é relativamente compacto em $X$ para cada $t \in J$.

Para compreendermos melhor esses comentários, é necessário construirmos soluções aproximadas para o problema de valor inicial (2.1), e isso é possível apenas com a continuidade da função $f$.

Inicialmente temos um lema que garante que tal função $f$ pode ser aproximada por funções localmente Lipschitzianas. Sua demonstração pode ser encontrada em [11], pág. 5, Lema 1.1.

Lema 2.2.3 Sejam $X$ e $Y$ espaços de Banach, $\Omega \subset X$ um aberto e $f: \Omega \longrightarrow Y$ uma função contínua. Então, para cada $\epsilon>0$, existe $f_{\epsilon}: \Omega \longrightarrow Y$ localmente Lipschitziana tal que

$$
\sup _{\Omega}\left\|f(x)-f_{\epsilon}(x)\right\| \leqslant \epsilon .
$$

A seguir, enunciaremos o Teorema de Dugundji. Sua demonstração pode ser encontrada em [13], pág. 188, Teorema 6.1. Mas antes, precisamos da definição a seguir. 
Definição 2.2.4 Se A é um subconjunto de um espaço vetorial $Y$, o conjunto co $(A)$, chamado casco convexo de $A$, é a intersecção de todos os conjuntos convexos contendo A. Se Y é um espaço vetorial topológico, o conjunto $\overline{c o}(A)$, chamado casco convexo fechado de A, é a intersecção de todos conjuntos convexos fechados contendo $A$.

Algumas propriedades dos conjuntos $c o(A)$ e $\overline{c o}(A)$ podem ser encontradas em [14], pág. 415, Lemas 4 e 5.

Teorema 2.2.5 (Dugundji) Sejam $Z$ um espaço métrico, $\Omega \subset Z$ um subconjunto fechado não vazio, $Y$ um espaço normado e $f: \Omega \longrightarrow Y$ uma função contínua. Então existe uma extensão contínua $\tilde{f}: Z \longrightarrow Y$ de $f$ tal que:

(i) $\tilde{f}(Z) \subset \operatorname{co}(f(\Omega))$;

(ii) $\tilde{f}$ é localmente Lipschitziana sobre $Z \backslash \Omega$.

Na demonstração deste teorema, a propriedade (ii) não é mencionada. Entretanto, se na prova do teorema uma partição da unidade consistindo de funções localmente Lipschitzianas for usada, então a extensão contínua $\tilde{f}$ de $f$ também será localmente Lipschitziana sobre $Z \backslash \Omega$.

Utilizaremos o Lema 2.2.3 e o Teorema 2.2 .5 para encontrarmos soluções aproximadas para (2.1).

Teorema 2.2.6 (Soluções Aproximadas) Seja $I=\left[t_{0}, t_{0}+a\right]$, consideremos em $X$ a bola fechada $\bar{B}_{r}\left(x_{0}\right)=\left\{x:\left\|x-x_{0}\right\| \leqslant r\right\}, f: I \times \bar{B}_{r}\left(x_{0}\right) \longrightarrow X$ contínua $e$ $\|f(t, x)\| \leqslant M$ sobre $I \times \bar{B}_{r}\left(x_{0}\right)$ e tomemos $a_{\epsilon}=\min \left\{a, \frac{r}{M+\epsilon}\right\}$. Então existe uma função continuamente diferenciável $x_{\epsilon}:\left[t_{0}, a_{\epsilon}\right] \longrightarrow \bar{B}_{r}\left(x_{0}\right)$ tal que

$$
\begin{aligned}
& x_{\epsilon}^{\prime}=f\left(t, x_{\epsilon}\right)+y_{\epsilon}(t) \\
& x_{\epsilon}\left(t_{0}\right)=x_{0},
\end{aligned}
$$

$e\left\|y_{\epsilon}(t)\right\| \leqslant \epsilon$ sobre $\left[t_{0}, a_{\epsilon}\right]$

Demonstração: Como $I \times \bar{B}_{r}\left(x_{0}\right) \subset \mathbb{R} \times X$ é fechado e $f: I \times \bar{B}_{r}\left(x_{0}\right) \longrightarrow X$ é contínua, então, pelo Teorema 2.2.5, existe uma extensão contínua $\tilde{f}: \mathbb{R} \times X \longrightarrow X$ tal que $\|\tilde{f}(t, x)\| \leqslant M$ em quase todo lugar. Pelo Lema 2.2 .3 , existe $\tilde{f}_{\epsilon}: \mathbb{R} \times X \longrightarrow X$ localmente Lipschitziana tal que

$$
\left\|\tilde{f}_{\epsilon}(t, x)-\tilde{f}(t, x)\right\| \leqslant \epsilon, \quad \text { para todo }(t, x) \in \mathbb{R} \times X .
$$


Em particular, isto vale para $(t, x) \in I \times \bar{B}_{r}\left(x_{0}\right)$, logo

$$
\left\|\tilde{f}_{\epsilon}(t, x)-f(t, x)\right\| \leqslant \epsilon, \quad \text { para todo }(t, x) \in I \times \bar{B}_{r}\left(x_{0}\right) .
$$

Assim

$$
\left\|\tilde{f}_{\epsilon}(t, x)\right\| \leqslant\|f(t, x)\|+\epsilon \leqslant M+\epsilon .
$$

Consideremos $x_{\epsilon}$ sendo a solução do problema

$$
\begin{aligned}
& x^{\prime}=\tilde{f}_{\epsilon}(t, x) \\
& x\left(t_{0}\right)=x_{0} .
\end{aligned}
$$

Pelo Teorema 2.2.1, esta solução existe sobre $\left[t_{0}, a_{\epsilon}\right]$ e satisfaz

$$
x_{\epsilon}^{\prime}(t)=f\left(t, x_{\epsilon}(t)\right)+y_{\epsilon}(t)
$$

$\operatorname{com} y_{\epsilon}(t)=\tilde{f}_{\epsilon}\left(t, x_{\epsilon}(t)\right)-f\left(t, x_{\epsilon}(t)\right)$. Assim

$$
\left\|y_{\epsilon}(t)\right\|=\left\|\tilde{f}_{\epsilon}\left(t, x_{\epsilon}(t)\right)-f\left(t, x_{\epsilon}(t)\right)\right\| \leqslant \epsilon .
$$

Consideremos, agora, as soluções aproximadas $x_{\epsilon}$ no Teorema 2.2.6, onde $f$ é limitada sobre $I \times \bar{B}_{r}\left(x_{0}\right)$. Claramente $S=\left\{x_{\epsilon}: 0<\epsilon \leqslant 1\right\} \subset C\left(\left[t_{0}, a_{\epsilon}\right], X\right)$ é eqüicontínuo e limitado. Daí, o Teorema 2.2 .2 se aplica no caso em que a dimensão de $X$ é finita, pois neste caso $\bar{S}$ é compacto (uma vez que $S$ é limitado e $\bar{S}$ fechado), portanto $S(t)$ é relativamente compacto em $X$ para cada $t \in I$. Assim, existe uma subseqüência $\left(x_{\epsilon_{n}}\right)_{n \in \mathbb{N}}$ da seqüência de soluções aproximadas, convergindo para a solução $x$ do nosso problema de valor inicial (2.1). Entretanto, se a dimensão de $X$ for infinita, então um subconjunto limitado de $X$ não precisa ser relativamente compacto, pois fechado e limitado não implicam compacto. Isto indica que o Teorema de Peano pode ser falso em dimensão infinita, como já vimos nos Contra-Exemplos 1 e 2.

A seguir veremos um resultado mais geral nesta direção, que foi observado por Godunov em [18]. Mostraremos que todos os espaços de Banach em que o Teorema de Peano é verdadeiro são de dimensão finita. Para isso, como nos Contra-Exemplos 1 e 2 , construiremos uma função contínua $F: \mathbb{R} \times X \longrightarrow X$ tal que o problema de valor inicial

$$
\begin{aligned}
& x^{\prime}(t)=F(t, x(t)) \\
& x(0)=0,
\end{aligned}
$$


não possua solução em qualquer espaço de Banach $X$ de dimensão infinita. Isto é, a função $F$ construída contradiz o Teorema de Peano em qualquer espaço de Banach de dimensão infinita considerado. Antes de demonstrarmos este resultado precisamos de um lema, cuja prova pode ser encontrada em [8].

Lema 2.2.7 Seja X um espaço de Banach de dimensão infinita. Existem seqüências biortogonais $\left(e_{i}\right)$ e $\left(\alpha_{i}\right)$ em $X$ e $X^{*}$, respectivamente, tais que

(i) $\left\|e_{i}\right\|=\left\|\alpha_{i}\right\|=1$ para todo $i$

(ii) ( $\left.e_{i}\right)$ é uma base de Schauder (ver [26], pág. 68) para o subespaço linear fechado $L$ de $X$ gerado pelo conjunto de todos os $e_{i}$;

(iii) se $P_{m}(x)=\sum_{i=1}^{m} \alpha_{i}(x) e_{i}$, então $P_{m}$ é uma projeção em $L$ com norma menor do que $1+\frac{1}{m}$ para um número natural $m$ arbitrário.

Então já podemos mostrar o seguinte resultado:

Teorema 2.2.8 Todo espaço de Banach em que o Teorema de Peano é verdadeiro é de dimensão finita.

Demonstração: Seja $X$ um espaço de Banach de dimensão infinita. Então existem sequiências biortogonais $\left(e_{n}\right)$ e $\left(\alpha_{n}\right)$ em $X$ e $X^{*}$, respectivamente, e um subespaço fechado $L$ de $X$ com as propriedades descritas no Lema 2.2.7. Consideremos uma função $f: \mathbb{R} \times L \longrightarrow L$ dada por

$$
f(t, x)= \begin{cases}\frac{P(t, x)}{\sqrt{\|P(t, x)\|}}+\varphi(t, x), & \text { se } P(t, x) \neq 0 \\ \varphi(t, x), & \text { se } P(t, x)=0 .\end{cases}
$$

A função $P: \mathbb{R} \times L \longrightarrow L$ é dada pela fórmula

$$
P(t, x)=\sum_{n=1}^{\infty} \psi_{n}(t) \alpha_{n}(x) e_{n}
$$

onde $\psi_{n}: \mathbb{R} \longrightarrow \mathbb{R}$ é tal que $\psi_{n}(t)$ é linear sobre o intervalo $\left[c_{n}, b_{n}\right]$, e

$$
\psi_{n}(t)= \begin{cases}0, & \text { se } t \leqslant c_{n} \\ 1, & \text { se } t \geqslant b_{n}\end{cases}
$$


onde

$$
a_{n}=\frac{1}{2 n+1}, \quad b_{n}=\frac{1}{2 n} \quad \text { e } \quad c_{n}=\frac{a_{n}+b_{n}}{2} .
$$

Definimos a função $\varphi: \mathbb{R} \times L \longrightarrow L$ por

$$
\varphi(t, x)=\sum_{n=1}^{\infty} \varphi_{n}(t) h\left(\frac{\left(t-b_{n+1}\right)^{2}}{4}-\left\|x-P_{n}(x)\right\|\right) e_{n}
$$

onde $\varphi_{n}: \mathbb{R} \longrightarrow \mathbb{R}$ satisfaz as seguintes condições: $\varphi_{n}(t)=0$, para $\left.t \notin\right] a_{n}, c_{n}[\mathrm{e}$ $0<\varphi_{n}(t)<\frac{1}{n}$, para $\left.t \in\right] a_{n}, c_{n}[$. A função $h: \mathbb{R} \longrightarrow \mathbb{R}$ definimos por

$$
h(t)= \begin{cases}0, & \text { para } t<0 \\ t, & \text { para } t \in[0,1] \\ 1, & \text { para } t>1 .\end{cases}
$$

Provaremos que a função $f$ é contínua em todos os pontos $\left(t_{0}, x_{0}\right)$ de $\mathbb{R} \times L$. Para isto, mostraremos que as funções $\varphi$ e $P$ são contínuas.

Uma vez que $P_{n}(x)$ é uma projeção em $L$ e $\alpha_{n}(x)$ é um funcional linear contínuo, para todo $n$, então as funções $\varphi$ e $P$ são contínuas em $x_{0}$. Assim, $f$ é contínua em $x_{0}$. Mostraremos, a seguir, a continuidade de $\varphi$ e $P$ em $t_{0}$.

- $\varphi$ é contínua em $t_{0}$ : se $t_{0} \in \mathbb{R} \backslash[0,1]$, então $\varphi_{n}\left(t_{0}\right)=0$. E como $\varphi_{n}$ é contínua em $t_{0}$, então para $t$ próximo de $t_{0}$,

$$
\left\|\varphi(t, x)-\varphi\left(t_{0}, x\right)\right\|=0
$$

e a continuidade de $\varphi$ em $t_{0}$ é imediata.

Se $t_{0} \in[0,1]$, então existe um único $n$ tal que $\left.t_{0} \in\right] \frac{1}{2 n+1}, \frac{1}{2 n}[=] a_{n}, b_{n}[$. Logo para $t$ próximo de $t_{0}$,

$$
\begin{aligned}
& \left\|\varphi(t, x)-\varphi\left(t_{0}, x\right)\right\|=\| \sum_{k=1}^{\infty} \varphi_{k}(t) h\left(\frac{\left(t-b_{k+1}\right)^{2}}{4}-\left\|x-P_{k}(x)\right\|\right) e_{k} \\
& -\sum_{k=1}^{\infty} \varphi_{k}\left(t_{0}\right) h\left(\frac{\left(t_{0}-b_{k+1}\right)^{2}}{4}-\left\|x-P_{k}(x)\right\|\right) e_{k} \| \\
& =\| \sum_{k=1}^{n-1} \varphi_{k}(t) h\left(\frac{\left(t-b_{k+1}\right)^{2}}{4}-\left\|x-P_{k}(x)\right\|\right) e_{k}
\end{aligned}
$$




$$
\begin{aligned}
& +\varphi_{n}(t) h\left(\frac{\left(t-b_{n+1}\right)^{2}}{4}-\left\|x-P_{n}(x)\right\|\right) e_{n} \\
& +\sum_{k=n+1}^{\infty} \varphi_{k}(t) h\left(\frac{\left(t-b_{k+1}\right)^{2}}{4}-\left\|x-P_{k}(x)\right\|\right) e_{k} \\
& -\sum_{k=1}^{n-1} \varphi_{k}\left(t_{0}\right) h\left(\frac{\left(t_{0}-b_{k+1}\right)^{2}}{4}-\left\|x-P_{k}(x)\right\|\right) e_{k} \\
& -\varphi_{n}\left(t_{0}\right) h\left(\frac{\left(t_{0}-b_{n+1}\right)^{2}}{4}-\left\|x-P_{n}(x)\right\|\right) e_{n} \\
& -\sum_{k=n+1}^{\infty} \varphi_{k}\left(t_{0}\right) h\left(\frac{\left(t_{0}-b_{k+1}\right)^{2}}{4}-\left\|x-P_{k}(x)\right\|\right) e_{k} \| .
\end{aligned}
$$

Examinaremos como se comportam as $\varphi_{k}(t)$ 's para $\left.t \in\right] a_{n}, b_{n}$ [. Inicialmente, para $k<n$, temos $\frac{1}{2 n}<\frac{1}{2 k+1}$, isto é, $2 k+1<2 n$ e para $k>n$, temos $\frac{1}{2 k}<\frac{1}{2 n+1}$. De fato, como $k<n$ e $k, n \in \mathbb{N}$, o máximo que poderia acontecer é que $k=n-1$ e, neste caso, teríamos $2 k+1=2(n-1)+1=2 n-1<2 n$, para todo $k, n \in \mathbb{N}$. O outro caso é análogo. Assim,

$$
\ldots<a_{n+1}<c_{n+1}<b_{n+1}<a_{n}<c_{n}<b_{n}<a_{n-1}<c_{n-1}<b_{n-1}<\ldots
$$

Portanto podemos notar que as $\varphi_{k}(t)$ 's, para $\left.t \in\right] a_{n}, b_{n}[$, possuem o seguinte comportamento

$$
\varphi_{k}(t)= \begin{cases}0, & \text { se } k<n \\ \varphi_{n}(t), & \text { se } k=n \\ 0, & \text { se } k>n\end{cases}
$$

Logo (2.31) fica

$$
\begin{aligned}
& \left\|\varphi(t, x)-\varphi\left(t_{0}, x\right)\right\| \\
= & \left\|\varphi_{n}(t) h\left(\frac{\left(t-b_{n+1}\right)^{2}}{4}-\left\|x-P_{n}(x)\right\|\right) e_{n}-\varphi_{n}\left(t_{0}\right) h\left(\frac{\left(t_{0}-b_{n+1}\right)^{2}}{4}-\left\|x-P_{n}(x)\right\|\right) e_{n}\right\| \\
\leqslant & \left\|\varphi_{n}(t) h\left(\frac{\left(t-b_{n+1}\right)^{2}}{4}-\left\|x-P_{n}(x)\right\|\right)-\varphi_{n}\left(t_{0}\right) h\left(\frac{\left(t_{0}-b_{n+1}\right)^{2}}{4}-\left\|x-P_{n}(x)\right\|\right)\right\|\left\|e_{n}\right\| .
\end{aligned}
$$

Como $\varphi_{n}$ e $h$ são contínuas em $t_{0}$, então

$$
\left\|\varphi(t, x)-\varphi\left(t_{0}, x\right)\right\| \rightarrow 0, \quad \text { quando } t \rightarrow t_{0}
$$


No caso em que $t_{0}=0$, temos $\varphi_{n}\left(t_{0}\right)=0$. E para $t$ próximo de $t_{0}=0$, segue que $t \in] \frac{1}{2 n+1}, \frac{1}{2 n}[$. Logo

$$
\begin{aligned}
\|\varphi(t, x)-\varphi(0, x)\| & =\left\|\sum_{k=1}^{\infty} \varphi_{k}(t) h\left(\frac{\left(t-b_{k+1}\right)^{2}}{4}-\left\|x-P_{k}(x)\right\|\right) e_{k}\right\| \\
& =\left\|\varphi_{n}(t) h\left(\frac{\left(t-b_{n+1}\right)^{2}}{4}-\left\|x-P_{n}(x)\right\|\right) e_{n}\right\| .
\end{aligned}
$$

E como $\varphi_{n}$ e $h$ são contínuas em $t_{0}=0$, então $\varphi$ é contínua em $t_{0}=0$.

Portanto $\varphi$ é contínua em $t_{0}$.

- $P$ é contínua em $t_{0}$ : se $t_{0} \in \mathbb{R} \backslash[0,1]$, então $\psi_{n}\left(t_{0}\right)=0$ para $t_{0}<0$ e $\psi_{n}\left(t_{0}\right)=1$ para $t_{0}>1$, e como $\psi_{n}$ é contínua em $t_{0}$, então para $t$ próximo de $t_{0}$ temos, em ambos os casos,

$$
\left\|P(t, x)-P\left(t_{0}, x\right)\right\|=0,
$$

e a continuidade de $P$ em $t_{0} \in \mathbb{R} \backslash[0,1]$ é imediata.

Agora se $t_{0} \in[0,1]$, então existe um único $n$ tal que $\left.t_{0} \in\right] a_{n}, b_{n}[=] \frac{1}{2 n+1}, \frac{1}{2 n}[$. Logo para $t$ próximo de $t_{0}$,

$$
\begin{aligned}
& \left\|P(t, x)-P\left(t_{0}, x\right)\right\|=\left\|\sum_{k=1}^{\infty} \psi_{k}(t) \alpha_{k}(x) e_{k}-\sum_{k=1}^{\infty} \psi_{k}\left(t_{0}\right) \alpha_{k}(x) e_{k}\right\| \\
& =\| \sum_{k=1}^{n-1} \psi_{k}(t) \alpha_{k}(x) e_{k}+\psi_{n}(t) \alpha_{n}(x) e_{n}+\sum_{k=n+1}^{\infty} \psi_{k}(t) \alpha_{k}(x) e_{k} \\
& -\sum_{k=1}^{n-1} \psi_{k}\left(t_{0}\right) \alpha_{k}(x) e_{k}-\psi_{n}\left(t_{0}\right) \alpha_{n}(x) e_{n}-\sum_{k=n+1}^{\infty} \psi_{k}\left(t_{0}\right) \alpha_{k}(x) e_{k} \| .
\end{aligned}
$$

De maneira análoga ao caso anterior das $\varphi_{k}(t)$ 's, segue que para $\left.t \in\right] a_{n}, b_{n}[$ as $\psi_{k}(t)$ 's se comportam da seguinte maneira

$$
\psi_{k}(t)= \begin{cases}0, & \text { se } k<n \\ \psi_{n}(t), & \text { se } k=n \\ 1, & \text { se } k>n\end{cases}
$$


Logo (2.32) fica

$$
\begin{aligned}
& \left\|P(t, x)-P\left(t_{0}, x\right)\right\|=\| \psi_{n}(t) \alpha_{n}(x) e_{n}+\sum_{k=n+1}^{\infty} \alpha_{k}(x) e_{k}-\psi_{n}\left(t_{0}\right) \alpha_{n}(x) e_{n} \\
& -\sum_{k=n+1}^{\infty} \alpha_{k}(x) e_{k}\|=\|\left(\psi_{n}(t)-\psi_{n}\left(t_{0}\right)\right) \alpha_{n}(x) e_{n} \|=\left|\psi_{n}(t)-\psi_{n}\left(t_{0}\right)\right| .
\end{aligned}
$$

Como $\psi_{n}$ é contínua em $\left.t_{0} \in\right] a_{n}, b_{n}\left[\right.$, então $P$ é contínua em $\left.t_{0} \in\right] a_{n}, b_{n}[$. Se $t_{0}=\frac{1}{2 n}$, com $n=1,2, \ldots$, a prova é análoga.

No caso em que $t_{0}=0$, temos $\psi_{n}\left(t_{0}\right)=0$. Como $\left(\alpha_{n}\right)$ é uma seqüência de funcionais lineares contínuos, então dado $\epsilon>0$ existe $n_{0} \in \mathbb{N}$ tal que

$$
\left\|\sum_{k=n}^{\infty} \alpha_{k}(x) e_{k}\right\|<\frac{\epsilon}{2} \quad \text { e } \quad\left\|\alpha_{n}(x)\right\|<\frac{\epsilon}{2}, \quad \text { para todo } n \geqslant n_{0} .
$$

Neste caso, para $t$ próximo de $t_{0}=0$, segue que $\left.t \in\right] \frac{1}{2 n+1}, \frac{1}{2 n}\left[\right.$ para $n \geqslant n_{0}$. Logo

$$
\begin{aligned}
\|P(t, x)-P(0, x)\| & =\left\|\sum_{k=1}^{\infty} \psi_{k}(t) \alpha_{k}(x) e_{k}-\sum_{k=1}^{\infty} \psi_{k}(0) \alpha_{k}(x) e_{k}\right\| \\
& =\left\|\psi_{n}(t) \alpha_{n}(x) e_{n}+\sum_{k=n+1}^{\infty} \alpha_{k}(x) e_{k}\right\| \\
& \leqslant\left\|\psi_{n}(t)\right\|\left\|\alpha_{n}(x)\right\|\left\|e_{n}\right\|+\left\|\sum_{k=n+1}^{\infty} \alpha_{k}(x) e_{k}\right\|<\epsilon .
\end{aligned}
$$

Portanto $P$ é contínua em $t_{0}=0$.

Mostraremos, agora, a continuidade de $f$ em $t_{0}$.

Se $P(t, x)=0$, então a continuidade de $f$ segue da continuidade de $\varphi$.

Se $P(t, x) \neq 0$, então, tomando uma seqüência $\left(t_{n}\right)_{n \geqslant 0}$ tal que $t_{n} \rightarrow t_{0}$ quando $n \rightarrow \infty$, segue que $f\left(t_{n}, x\right) \rightarrow f\left(t_{0}, x\right)$. De fato,

$$
\left\|f\left(t_{n}, x\right)-f\left(t_{0}, x\right)\right\| \leqslant\left\|\frac{P\left(t_{n}, x\right)}{\sqrt{\left\|P\left(t_{n}, x\right)\right\|}}-\frac{P\left(t_{0}, x\right)}{\sqrt{\left\|P\left(t_{0}, x\right)\right\|}}\right\|+\left\|\varphi\left(t_{n}, x\right)-\varphi\left(t_{0}, x\right)\right\| .
$$

Logo $\left\|f\left(t_{n}, x\right)-f\left(t_{0}, x\right)\right\| \rightarrow 0$ quando $n \rightarrow \infty$, uma vez que $\varphi$ e $P$ são contínuas em $t_{0}$. 
Portanto, a função $f$ é contínua sobre $\mathbb{R} \times L$. Logo, pelo Teorema 2.2.5, a função $f: \mathbb{R} \times L \longrightarrow L$ possui uma extensão contínua $F: \mathbb{R} \times X \longrightarrow L$. Mostraremos então que o problema de Cauchy

$$
\begin{aligned}
& x^{\prime}(t)=F(t, x(t)) \\
& x(0)=0,
\end{aligned}
$$

não tem uma solução em qualquer vizinhança de zero. Suponhamos, por contradição, que uma função $x: \mathbb{R} \longrightarrow X$, definida em uma vizinhança de zero, seja uma solução de (2.33). Então

$$
x(t)=\int_{0}^{t} F(s, x(s)) d s .
$$

Mas $F(s, x(s)) \in L$, com $L$ subespaço fechado em $X$, portanto $x(t) \in L$ para cada $t$. Conseqüentemente, $x(t)$ é uma solução do problema

$$
\begin{aligned}
& x^{\prime}(t)=f(t, x(t)) \\
& x(0)=0 .
\end{aligned}
$$

Temos duas possibilidades para a função $x(t)$ :

$1^{\mathrm{a}}$ ) existe $n$ arbitrariamente grande tal que $x\left(b_{n}\right) \neq 0$;

$2^{\mathrm{a}}$ ) para todo $n$ suficientemente grande, temos $x\left(b_{n}\right)=0$.

Analisemos a primeira possibilidade.

Escolhemos $m$ tal que $x\left(b_{m}\right) \neq 0$. Consideremos o problema de Cauchy

$$
\begin{aligned}
& u^{\prime}(t)=f(t, u(t)) \\
& u\left(b_{m}\right)=x\left(b_{m}\right) .
\end{aligned}
$$

Claramente $x(t)$ é uma solução de (2.34). Mostraremos que a única solução de (2.34) sobre $\left[b_{m}, 1\right]$ é a função

$$
u(t)=\frac{x\left(b_{m}\right)}{\left\|x\left(b_{m}\right)\right\|} \cdot \frac{\left(t-\tilde{b_{m}}\right)^{2}}{4},
$$

onde $\tilde{b_{m}}$ satisfaz as condições $\tilde{b_{m}}<b_{m}$ e $\left(b_{m}-\tilde{b_{m}}\right)^{2}=4\left\|x\left(b_{m}\right)\right\|$.

Claramente a função $u(t)$ satisfaz a condição inicial em (2.34). Provaremos que $u^{\prime}(t)=f(t, u(t))$. Se $n<m$ e $t<b_{m}$, então $\alpha_{n}(f(t, x(t)))=0$. De fato, se $n<m$, já 
vimos que $\ldots<a_{m}<c_{m}<b_{m}<a_{n}<c_{n}<b_{n}<\ldots$. Logo para $t<b_{m}, \psi_{n}(t)=0$ e $\varphi_{n}(t)=0$ para $n<m$. Então

$$
P(t, x)=\sum_{n=m}^{\infty} \psi_{n}(t) \alpha_{n}(x) e_{n}
$$

e

$$
\varphi(t, x)=\sum_{n=m}^{\infty} \varphi_{n}(t) h\left(\frac{\left(t-b_{n+1}\right)^{2}}{4}-\left\|x-P_{n}(x)\right\|\right) e_{n} .
$$

Uma vez que $f(t, x(t))$ é dada por (2.30) e $f(t, x(t)) \in L$, com $\left(e_{n}\right)$ uma base de Schauder para $L$, temos

$$
f(t, x(t))=\sum_{n=1}^{\infty} \alpha_{n}(f(t, x(t))) e_{n}=\sum_{n=m}^{\infty} \alpha_{n}(f(t, x(t))) e_{n} .
$$

Assim $\alpha_{n}(f(t, x(t)))=0$ para $n<m$ e $t<b_{m}$.

Agora para $n<m$, uma vez que

$$
x\left(b_{m}\right)=\int_{0}^{b_{m}} f(t, x(t)) d t, \quad \text { (integral de Riemann) }
$$

e $\alpha_{n}$ é linear, com $\alpha_{n}(f(t, x(t)))=0$ para $t<b_{m}$, então $\alpha_{n}\left(x\left(b_{m}\right)\right)=0$ e por (2.35), temos

$$
\alpha_{n}(u(t))=\alpha_{n}\left[\frac{x\left(b_{m}\right)}{\left\|x\left(b_{m}\right)\right\|} \frac{\left(t-\tilde{b_{m}}\right)^{2}}{4}\right]=\frac{\left(t-\tilde{b_{m}}\right)^{2}}{4\left\|x\left(b_{m}\right)\right\|} \alpha_{n}\left(x\left(b_{m}\right)\right)=0 .
$$

Portanto $\alpha_{n}(u(t))=0$ para todo $t$ e $n<m$. Conseqüentemente, para $t>b_{m}$, existe $k<m$ tal que $t \in\left[a_{k}, b_{k}\right]$. Logo

$$
\begin{aligned}
P(t, u(t)) & =\sum_{n=1}^{\infty} \psi_{n}(t) \alpha_{n}(u(t)) e_{n}=\sum_{n=1}^{m-1} \psi_{n}(t) \alpha_{n}(u(t)) e_{n}+\sum_{n=m}^{\infty} \psi_{n}(t) \alpha_{n}(u(t)) e_{n} \\
& =\sum_{n=m}^{\infty} \alpha_{n}(u(t)) e_{n}=\sum_{n=1}^{\infty} \alpha_{n}(u(t)) e_{n}-\sum_{n=1}^{m-1} \alpha_{n}(u(t)) e_{n}
\end{aligned}
$$

Como $u(t) \in L$, então podemos escrever $u(t)=\sum_{n=1}^{\infty} \alpha_{n}(u(t)) e_{n}$. De (2.36) temos

$$
P(t, u(t))=u(t)-\sum_{n=1}^{m-1} \alpha_{n}(u(t)) e_{n}=u(t)
$$

E ainda, $\varphi(t, u(t))=0$ para $t \geqslant b_{m}$. De fato, para $t \geqslant b_{m}$, existe $k<m$ tal que $b_{k+1} \leqslant t \leqslant b_{k}, \operatorname{logo} \varphi_{n}(t)=0$ para todo $n \neq k$. E ainda temos duas possibilidades 
a considerar: $t \notin\left[a_{k}, c_{k}\right]$ ou $t \in\left[a_{k}, c_{k}\right]$. Se $t \notin\left[a_{k}, c_{k}\right] \dot{e}$ uma vez que por definição $\varphi_{k}(t)=0$, então $\varphi(t, u(t))=0$ para $t \geqslant b_{m}$. Agora, se $t \in\left[a_{k}, c_{k}\right]$, com $k<m$, mostraremos que $h=0$. Sabemos que

$$
\varphi(t, u(t))=\varphi_{k}(t) h\left(\frac{\left(t-b_{k+1}\right)^{2}}{4}-\left\|\frac{x\left(b_{m}\right)}{\left\|x\left(b_{m}\right)\right\|} \cdot \frac{\left(t-\tilde{b_{m}}\right)^{2}}{4}-P_{k}(u(t))\right\|\right) e_{k} .
$$

Mas

$$
P_{k}(u(t))=\sum_{i=1}^{k} \alpha_{i}(u(t)) e_{i}=0
$$

uma vez que $k<m, 1 \leqslant i \leqslant k$ e $\alpha_{i}(u(t))=0$ para $i<m$. Assim

$$
\varphi(t, u(t))=\varphi_{k}(t) h\left(\frac{\left(t-b_{k+1}\right)^{2}}{4}-\frac{\left(t-\tilde{b_{m}}\right)^{2}}{4}\right) e_{k} .
$$

Como $\tilde{b_{m}}<b_{m} \leqslant b_{k+1}$ segue que $\frac{\left(t-b_{k+1}\right)^{2}}{4}-\frac{\left(t-\tilde{b_{m}}\right)^{2}}{4}<0$. Logo $h=0$ e, portanto, $\varphi(t, u(t))=0$ para $t \geqslant b_{m}$.

Com base nos cálculos anteriores, temos

$$
f(t, u(t))=\frac{P(t, u(t))}{\sqrt{\|P(t, u(t))\|}}=\frac{u(t)}{\sqrt{\|u(t)\|}}=\frac{x\left(b_{m}\right)}{\left\|x\left(b_{m}\right)\right\|} \cdot \frac{2\left(t-\tilde{b_{m}}\right)}{4}=u^{\prime}(t) .
$$

Portanto, $u(t)$ é uma solução de (2.34). A unicidade da solução de (2.34) segue do fato que a função $f$ é localmente Lipschitziana na segunda variável em uma vizinhança do conjunto $M=\left\{(t, u(t)): t \in\left[b_{m}, 1\right]\right\} \subset \mathbb{R} \times L$. De fato, $P(t, x)$ é Lipschitziana na segunda variável para $t>0$, uma vez que para um arbitrário $n$ sabemos que se $t \in\left[b_{n+1}, b_{n}\right], \alpha_{n}$ é linear e $\left(\alpha_{n}\right)$ e $\left(e_{n}\right)$ são seqüências biortogonais, então

$$
\begin{aligned}
& \|P(t, x)-P(t, y)\| \leqslant\left\|\psi_{n}(t) \alpha_{n}(x-y) e_{n}\right\|+\left\|\sum_{k=n+1}^{\infty} \alpha_{k}(x-y) e_{k}\right\| \\
& =\left\|\psi_{n}(t) \alpha_{n}(x-y) e_{n}\right\|+\left\|\sum_{k=1}^{\infty} \alpha_{k}(x-y) e_{k}-\sum_{k=1}^{n} \alpha_{k}(x-y) e_{k}\right\| \\
& =\left\|\psi_{n}(t) \alpha_{n}(x-y) e_{n}\right\|+\left\|(x-y)-P_{n}(x-y)\right\| \\
& \leqslant\|x-y\|+\|x-y\|+1+\frac{1}{n} \leqslant 4\|x-y\| .
\end{aligned}
$$

Uma vez que $P(t, x) \neq 0$ em uma vizinhança de $M$, segue que $\frac{P(t, x)}{\sqrt{\|P(t, x)\|}}$ é localmente Lipschitziana nesta vizinhança. E como também em uma vizinhança de $M$ 
temos $\varphi=0$. Então $f$ é localmente Lipschitziana na segunda variável. Daí, pelo Teorema 2.2.1, $u(t)=x(t)$.

Se $t>b_{m}$ e $n<m$, então $\alpha_{n}(x(t))=\alpha_{n}(u(t))=0$. Uma vez que $m$ pode ser escolhido arbitrariamente grande e $\left(e_{n}\right)$ é uma base de Schauder em $L, \operatorname{com} x(t) \in L$, então $x(t)=0$. Esta contradição mostra que a primeira possibilidade é impossível.

Analisemos a segunda possibilidade.

Escolhemos $m$ tal que $x\left(b_{m}\right)=0$. Se $n>m$ e $t \in\left[b_{m+1}, b_{m}\right]$, então

$$
\frac{d}{d t} \alpha_{n}(x(t))=\frac{\alpha_{n}(x(t))}{\sqrt{\|P(t, x(t))\|}}
$$

De fato, sabemos que

$$
\varphi(t, x)=\varphi_{m}(t) h\left(\frac{\left(t-b_{m+1}\right)^{2}}{4}-\left\|x-P_{m}(x)\right\|\right) e_{m}
$$

e

$$
P(t, x)=\psi_{m} \alpha_{m}(x) e_{m}+\sum_{n=m+1}^{\infty} \alpha_{n}(x) e_{n} .
$$

Assim,

$$
f(t, x)=\frac{P(t, x)}{\sqrt{\|P(t, x)\|}}, \quad \text { para } n>m .
$$

Mas $x(t)=\sum_{n=1}^{\infty} \alpha_{n}(x(t)) e_{n}$, logo para $n>m$

$$
x^{\prime}(t)=\sum_{n=1}^{\infty} \frac{d}{d t} \alpha_{n}(x(t)) e_{n} .
$$

Por outro lado,

$$
x^{\prime}(t)=f(t, x(t))=\frac{\sum_{n=m+1}^{\infty} \alpha_{n}(x(t)) e_{n}}{\sqrt{\|P(t, x(t))\|}} .
$$

Daí obtemos (2.38).

Suponhamos que existe $n>m$ tal que $\alpha_{n}\left(x\left(t_{0}\right)\right) \neq 0$ para algum $t_{0} \in\left[b_{m+1}, b_{m}\right]$. Se $\alpha_{n}\left(x\left(t_{0}\right)\right)>0$, então por (2.38), $\frac{d}{d t} \alpha_{n}\left(x\left(t_{0}\right)\right)>0$. Logo em uma vizinhança de $t_{0}$, $\alpha_{n}(x(t))$ é crescente. Assim $\alpha_{n}\left(x\left(b_{m}\right)\right)>\alpha_{n}\left(x\left(t_{0}\right)\right)>0$. E se $\alpha_{n}\left(x\left(t_{0}\right)\right)<0$, então por (2.38), $\frac{d}{d t} \alpha_{n}\left(x\left(t_{0}\right)\right)<0$. Logo em uma vizinhança de $t_{0}, \alpha_{n}(x(t))$ é decrescente. Assim $\alpha_{n}\left(x\left(b_{m}\right)\right)<\alpha_{n}\left(x\left(t_{0}\right)\right)<0$, o que é um absurdo, logo $\alpha_{n}(x(t))=0$ para todo $t \in\left[b_{m+1}, b_{m}\right]$ e $n>m$. Portanto, para $n=m$,

$$
\frac{d}{d t} \alpha_{m}(x(t))=\frac{\psi_{m}(t) \alpha_{m}(x(t))}{\sqrt{\|P(t, x(t))\|}}+\varphi_{m}(t) h\left(\frac{\left(t-b_{m+1}\right)^{2}}{4}-\left\|x(t)-P_{m}(x(t))\right\|\right) .
$$


Olhando $\psi_{m}(t)$ para $t \in\left[0, c_{m}\right]$ temos $\psi_{m}(t)=0$. Temos também

$$
x(t)=\sum_{n=1}^{m} \alpha_{n}(x(t)) e_{n}=P_{m}(x(t)),
$$

uma vez que $\alpha_{n}(x(t))=0$ para $n>m$. Logo

$$
\frac{d}{d t} \alpha_{m}(x(t))=\varphi_{m}(t) h\left(\frac{\left(t-b_{m+1}\right)^{2}}{4}\right), \quad \text { sobre }\left[0, c_{m}\right] .
$$

Olhando, agora, para o intervalo $\left[0, a_{m}\right]$ temos $\varphi_{m}=0$. Então

$$
\frac{d}{d t} \alpha_{m}(x(t))=0, \quad \text { sobre }\left[0, a_{m}\right] .
$$

Logo $\alpha_{m}(x(t))$ é constante sobre $\left[0, a_{m}\right]$. E ainda, sobre $] a_{m}, c_{m}\left[\right.$ temos $\varphi_{m}>0$. Assim

$$
\left.\frac{d}{d t} \alpha_{m}(x(t))>0, \quad \text { sobre }\right] a_{m}, c_{m}[\text {. }
$$

Daí, $\alpha_{m}(x(t))$ é crescente em $] a_{m}, c_{m}[$. Logo

$$
\alpha_{m}\left(x\left(c_{m}\right)\right)>\alpha_{m}\left(x\left(a_{m}\right)\right)=\alpha_{m}(x(0))=\alpha_{m}(0)=0
$$

$\operatorname{assim} \alpha_{m}\left(x\left(c_{m}\right)\right)>0$. Uma vez que $\alpha_{m}(x(t))$ é contínua em $\left[a_{m}, b_{m}\right]$, segue que $\alpha_{m}\left(x\left(b_{m}\right)\right)>0$, mas isto é um absurdo, pois $x\left(b_{m}\right)=0$. Assim a segunda possibilidade é impossível.

Portanto, o problema (2.33) não possui solução e, conseqüentemente, o Teorema de Peano não é verdadeiro.

\subsubsection{Unicidade e Dependência Contínua em Dimensão In- finita}

No caso em que um espaço de Banach $X$ tem dimensão finita, sabemos que se $\tilde{f}: \mathbb{R} \times X \longrightarrow X$ é uma função contínua em um conjunto aberto $\Omega$ de $\mathbb{R} \times X$ tal que para cada $\left(t_{0}, x_{0}\right) \in \Omega$ a equação diferencial

$$
x^{\prime}=\tilde{f}(t, x)
$$

tem uma solução $x(t)$ com $x\left(t_{0}\right)=x_{0}$, definida sobre $J$, onde $J$ é o intervalo maximal de existência, e ainda, não existe outra solução satisfazendo $x\left(t_{0}\right)=x_{0}$. Então 
esta solução depende continuamente do dado inicial $\left(t_{0}, x_{0}\right)$, isto é, para cada $t \in J$, $x\left(t ; t_{0}, u\right) \rightarrow x\left(t ; t_{0}, u_{0}\right)$ quando $u \rightarrow u_{0}$, onde $x\left(\cdot ; t_{0}, v\right)$ denota a solução de $(2.39)$ satisfazendo $x\left(t_{0} ; t_{0}, v\right)=v$. Assim, podemos dizer que a unicidade de soluções implica sua dependência contínua em relação ao dado inicial. Entretanto, se a dimensão de $X$ for infinita, esta propriedade pode não valer, como mostrado por Schäffer através de um contra-exemplo de uma equação diferencial em $l^{\infty}$ com unicidade, mas sem dependência contínua. Neste trabalho, não veremos este contra-exemplo, mas o mesmo pode ser encontrado em [35]. Iremos nos dedicar a um resultado mais geral, que assegura que todos os espaços de Banach em que a unicidade de soluções implica sua dependência contínua em relação ao dado inicial são de dimensão finita. Este resultado foi provado por Blasi e Pianigiani em [4], e a prova é uma adaptação da construção desenvolvida no Teorema 2.2.8.

Teorema 2.2.9 Todo espaço de Banach em que a unicidade de soluçôes implica sua dependência contínua em relação ao dado inicial é de dimensão finita.

Demonstração: Seja $X$ um espaço de Banach de dimensão infinita. Então existem sequiências biortogonais $\left(e_{n}\right)$ e $\left(\alpha_{n}\right)$ em $X$ e $X^{*}$, respectivamente, e um subespaço fechado $L$ de $X$ com as propriedades descritas no Lema 2.2.7.

Seja $P: \mathbb{R} \times L \longrightarrow L$ dada por

$$
P(t, x)=\sum_{n=1}^{\infty} \Phi_{n}(t) \alpha_{n}(x) e_{n},
$$

onde, para cada $n \in \mathbb{N}, \Phi_{n}: \mathbb{R} \longrightarrow \mathbb{R}$ é uma função contínua tal que $\Phi_{n}(t)=0$ se $t \leqslant \frac{1}{n+1}, \Phi_{n}(t)=1$ se $t \geqslant \frac{1}{n}$ e $\Phi_{n}$ é linear sobre o intervalo $\left[\frac{1}{n+1}, \frac{1}{n}\right]$. A função $P$ é análoga à que introduzimos no Teorema 2.2.8. Também de maneira análoga podemos mostrar que $P$ é contínua em cada ponto $\left(t_{0}, x_{0}\right) \in \mathbb{R} \times L$.

Consideremos $f: \mathbb{R} \times L \longrightarrow L$ como sendo

$$
f(t, x)= \begin{cases}\frac{P(t, x)}{\left[\frac{\|x\|^{2}+\|x\|}{2}+\frac{t^{2}}{8}\right]^{\frac{1}{2}}}, & \text { se }(t, x) \neq(0,0) \\ 0, & \text { se }(t, x)=(0,0) .\end{cases}
$$

Uma vez que $P$ é Lipschitziana, segue que $f$ é localmente Lipschitziana em $x$ sobre $(\mathbb{R} \times L) \backslash(0,0)$. Claramente, a função $f$ é contínua sobre $(\mathbb{R} \times L) \backslash(0,0)$. 
Mostremos então que $f$ é contínua em $(0,0)$. Uma vez que, por (2.37) do Teorema $2.2 .8,\|P(t, x)\| \leqslant 4\|x\|$, obtemos

$$
\|f(t, x)-f(0,0)\|=\left\|\frac{P(t, x)}{\left[\frac{\|x\|^{2}+\|x\|}{2}+\frac{t^{2}}{8}\right]^{\frac{1}{2}}}\right\| \leqslant \frac{4\|x\|}{\left[\frac{\|x\|^{2}+\|x\|}{2}+\frac{t^{2}}{8}\right]^{\frac{1}{2}}} .
$$

Portanto $f$ é contínua e limitada sobre $\mathbb{R} \times L$. Daí, pelo Teorema 2.2.5, a função $f$ tem uma extensão contínua e limitada $\tilde{f}: \mathbb{R} \times X \longrightarrow L$, que é localmente Lipschitziana fora de $\mathbb{R} \times L$.

Notemos que o subespaço linear fechado $\mathbb{R} \times L$ de $\mathbb{R} \times X$ é invariante pela equação diferencial em (2.39). Isto é, se $x: J \longrightarrow X$ denota uma solução arbitrária de (2.39) com $x\left(t_{0}\right)=x_{0} \in L$, então o conjunto $\{(t, x(t)): t \in J\}$ está contido em $\mathbb{R} \times L$. Uma vez que sobre $\mathbb{R} \times L$ as funções $f$ e $\tilde{f}$ coincidem, segue que qualquer solução de (2.39) com $x\left(t_{0}\right)=x_{0} \in L$ é também uma solução da equação diferencial

$$
x^{\prime}=f(t, x) .
$$

Mostraremos que, para qualquer $\left(t_{0}, x_{0}\right) \in \mathbb{R} \times X$, a equação diferencial (2.39) tem uma solução $x: \mathbb{R} \longrightarrow X \operatorname{com} x\left(t_{0}\right)=x_{0}$, e ainda, que não existe outra solução satisfazendo $x\left(t_{0}\right)=x_{0}$. Entretanto veremos que, para a equação diferencial (2.39), a dependência contínua de soluções em relação ao dado inicial falha.

Seja $\left(t_{0}, x_{0}\right) \in \mathbb{R} \times L$ e suponhamos que $x\left(t_{0}\right)=0$. Sabemos que a função $x(t) \equiv 0$ sobre $\mathbb{R}$ satisfaz $x\left(t_{0}\right)=0$ e é uma solução de (2.39). Seja $x: J \longrightarrow X$ outra solução de (2.39) satisfazendo $x\left(t_{0}\right)=0$ e suponhamos que $0 \in J$. Como $x$ também é uma solução de (2.40), temos

$$
x^{\prime}(t)=\frac{\sum_{n=1}^{\infty} \Phi_{n}(t) \alpha_{n}(x(t)) e_{n}}{\left[\frac{\|x(t)\|^{2}+\|x(t)\|}{2}+\frac{t^{2}}{8}\right]^{\frac{1}{2}}}, \quad \text { se } t \in J \text { e } t \neq 0,
$$

e $x^{\prime}(0)=0$. Daí, para cada $n \in \mathbb{N}$, tomando $u_{n}(t)=\alpha_{n}(x(t)), t \in J$, temos, de maneira análoga ao Teorema 2.2.8,

$$
u_{n}^{\prime}(t)=\frac{\Phi_{n}(t) u_{n}(t)}{\left[\frac{\|x(t)\|^{2}+\|x(t)\|}{2}+\frac{t^{2}}{8}\right]^{\frac{1}{2}}}, \quad \text { se } t \in J \text { e } t \neq 0,
$$

e $u_{n}^{\prime}(0)=0$. Seja $k_{n}: J \longrightarrow \mathbb{R}$ definida por

$$
k_{n}(0)=0, \quad k_{n}(t)=\frac{\Phi_{n}(t)}{\left[\frac{\|x(t)\|^{2}+\|x(t)\|}{2}+\frac{t^{2}}{8}\right]^{\frac{1}{2}}}, \quad \text { se } t \neq 0 .
$$


Claramente, $k_{n}$ é contínua sobre $J$. Uma vez que para todo $t \in J$ a função $u_{n}$ satisfaz $u_{n}^{\prime}(t)=k_{n}(t) u_{n}(t)$ e $u_{n}\left(t_{0}\right)=0$, segue que $u_{n}(t)=0$ é uma solução para cada $t \in J$. Como $u_{n}(t)=\alpha_{n}(x(t))$, então $x(t)=0$ para todo $t \in J$. Assim, a função identicamente nula sobre $\mathbb{R}$ é a única solução de (2.39) satisfazendo $x\left(t_{0}\right)=0$.

Seja $\left(t_{0}, x_{0}\right) \in \mathbb{R} \times L$ e suponhamos agora que $x_{0} \neq 0$. Uma vez que $f$ é contínua, limitada e, fora da origem, $f$ é localmente Lipschitziana em $x$, então, pelo Teorema 2.2.1, a equação diferencial (2.40) possui somente uma solução $x: \mathbb{R} \longrightarrow L$ (definida para todo $t \in \mathbb{R}$ ) satisfazendo a condição $x\left(t_{0}\right)=x_{0}$. E ainda, $x(t)$ é uma solução de (2.39) e é a única satisfazendo $x\left(t_{0}\right)=x_{0}$, pois o subespaço $\mathbb{R} \times L$ é invariante por (2.39) e sobre este subespaço as funções $\tilde{f}$ e $f$ coincidem.

Seja $\left(t_{0}, x_{0}\right) \in(\mathbb{R} \times X) \backslash(\mathbb{R} \times L)$. Uma vez que sobre este conjunto $\tilde{f}$ é contínua, limitada e localmente Lipschitziana, e ainda, o conjunto $(\mathbb{R} \times X) \backslash(\mathbb{R} \times L)$ é invariante por (2.39), então, pelo Teorema 2.2.1, a equação diferencial (2.39) tem uma única solução $x: \mathbb{R} \longrightarrow X$ (definida para todo $t \in \mathbb{R}$ ) satisfazendo $x\left(t_{0}\right)=x_{0}$.

Veremos agora que, para a equação diferencial (2.39), não temos dependência contínua de soluções em relação ao dado inicial. Com esta finalidade, para cada $n \in \mathbb{N}$, tomemos $x: \mathbb{R} \longrightarrow X$ como sendo a solução de (2.39) satisfazendo $x_{n}(0)=\frac{e_{n}}{n}$. Notemos que $x_{n}(0) \rightarrow 0$, quando $n \rightarrow \infty$.

Fixando $n \in \mathbb{N}$, para cada $t \in \mathbb{R}$ temos

$$
x_{n}^{\prime}(t)=\frac{\sum_{k=1}^{\infty} \Phi_{k}(t) \alpha_{k}\left(x_{n}(t)\right) e_{k}}{\left[\frac{\left\|x_{n}(t)\right\|^{2}+\left\|x_{n}(t)\right\|}{2}+\frac{t^{2}}{8}\right]^{\frac{1}{2}}} .
$$

Daí, para $k=1,2, \ldots$,

$$
\frac{d}{d t} \alpha_{k}\left(x_{n}(t)\right)=\frac{\Phi_{k}(t) \alpha_{k}\left(x_{n}(t)\right)}{\left[\frac{\left\|x_{n}(t)\right\|^{2}+\left\|x_{n}(t)\right\|}{2}+\frac{t^{2}}{8}\right]^{\frac{1}{2}}} .
$$

Como as seqüências $\left(e_{n}\right)$ e $\left(\alpha_{n}\right)$ são biortogonais e $x_{n}(0)=\frac{e_{n}}{n}$, então para $k \neq n$ temos $\alpha_{k}\left(x_{n}(0)\right)=0$, e assim, $\alpha_{k}\left(x_{n}(t)\right)=0$ para todo $t \in \mathbb{R}$. Para $k=n$ temos, para todo $t \in \mathbb{R}$,

$$
u_{n}^{\prime}(t)=\frac{\Phi_{n}(t) u_{n}(t)}{\left[\frac{u_{n}^{2}(t)+\left|u_{n}(t)\right|}{2}+\frac{t^{2}}{8}\right]^{\frac{1}{2}}}
$$

onde $u_{n}(t)=\alpha_{n}\left(x_{n}(t)\right), t \in \mathbb{R}$. 
Uma vez que $\Phi_{n}(t)=0$ para $t \leqslant \frac{1}{n+1}$, segue de $(2.41)$ que $u_{n}^{\prime}(t)=0$ para $0 \leqslant t \leqslant \frac{1}{n+1}$. Assim $u_{n}$ é constante em $\left[0, \frac{1}{n+1}\right]$, e como $u_{n}(0)=\frac{1}{n}$, então $u_{n}(t)=\frac{1}{n}$ em $\left[0, \frac{1}{n+1}\right]$. Em particular, $u_{n}\left(\frac{1}{n+1}\right)=\frac{1}{n}>0$. Agora, para $t>\frac{1}{n+1}, \Phi_{n}(t)$ é positiva e ainda $u_{n}(t)$ é contínua, logo $u_{n}^{\prime}(t)>0$ para $t>\frac{1}{n+1}$. Assim $u_{n}(t)$ é crescente para $t>\frac{1}{n+1}$, daí $u_{n}(t) \geqslant \frac{1}{n}$ para todo $t \geqslant 0$. Logo, $u_{n}(t) \geqslant \frac{t^{2}}{8}$ para cada $t \in\left[0, \frac{1}{n}\right]$. E ainda, $u_{n}(t) \geqslant \frac{t^{2}}{8}$ para todo $t \in[0,1]$. De fato, suponhamos, por contradição, que isto não aconteça. Então existem $t_{0}, t_{1} \in\left[\frac{1}{n}, 1\right]$, com $t_{0}<t_{1}$, tais que $u_{n}\left(t_{0}\right)=\frac{t_{0}^{2}}{8}$ e $u_{n}(t)<\frac{t^{2}}{8}$ se $\left.\left.t \in\right] t_{0}, t_{1}\right]$. Como para cada $t \in\left[t_{0}, t_{1}\right]$ temos $u_{n}^{2}(t)<u_{n}(t) \leqslant \frac{t^{2}}{8}$ e $\Phi_{n}(t)=1$, então

$$
u_{n}^{\prime}(t)=\frac{u_{n}(t)}{\left[\frac{u_{n}^{2}(t)+\left|u_{n}(t)\right|}{2}+\frac{t^{2}}{8}\right]}>\frac{u_{n}(t)}{\left[\frac{t^{2}}{16}+\frac{t^{2}}{16}+\frac{t^{2}}{8}\right]^{\frac{1}{2}}}=\frac{2}{t} u_{n}(t) .
$$

Integrando a desigualdade acima de $t_{0}$ a $t$ e usando a condição $u_{n}\left(t_{0}\right)=\frac{t_{0}^{2}}{8}$, segue que $u_{n}(t)>\frac{t^{2}}{8}$, com $\left.\left.t \in\right] t_{0}, t_{1}\right]$, o que é uma contradição.

Uma vez que $\alpha_{k}\left(x_{n}(t)\right)=0$ para todo $t \in \mathbb{R}$ e $k \neq n$, então para todo $n \in \mathbb{N}$, obtemos

$$
\left\|x_{n}(t)\right\|=\left\|\sum_{k=1}^{\infty} \alpha_{k}\left(x_{n}(t)\right) e_{k}\right\|=\left\|\alpha_{n}\left(x_{n}(t)\right) e_{n}\right\|=u_{n}(t) \geqslant \frac{t^{2}}{8}, \quad \text { para } t \in[0,1] .
$$

Portanto, para a equação (2.39) não vale a dependência contínua em relação ao dado inicial, uma vez que a função identicamente nula sobre $\mathbb{R}$ é uma solução de (2.39), com $x_{n}(0) \rightarrow 0$, quando $n \rightarrow \infty$, enquanto $x_{n}(t)$ não converge para zero para qualquer $t \in] 0,1]$.

\subsection{Existência Local e Unicidade de Soluções e De- pendência Contínua}

Vimos na seção anterior que apenas a continuidade de $f$ não é suficiente para garantir a existência de uma solução do problema de valor inicial (2.1) em um espaço de Banach $X$ de dimensão infinita. Por outro lado, se $f$ é localmente Lipschitziana em $x$, a existência local e unicidade de soluções estão asseguradas. Entretanto, sob estas condições, vimos na demonstração do Teorema 2.2 .9 que para espaços de Banach de dimensão infinita a unicidade de soluções não implica dependência contínua 
em relação ao dado inicial. Com base nesses fatos, nesta seção iremos estabelecer condições gerais sobre $f$ para garantir que o problema de valor inicial (2.1) seja bem posto, isto é, as soluções de (2.1) existem, são únicas e dependem continuamente do dado inicial. Nesta seção e nas Seções 2.4 e 2.5, utilizaremos [27].

No teorema a seguir novamente aparece o conceito de diferencial de Fréchet, dado na Definição 2.1.9, (iii). Algumas propriedades podem ser encontradas na Seção 2.1.

Teorema 2.3.1 Suponhamos que $f \in C\left(I \times \bar{B}_{b}\left(x_{0}\right), X\right)$, onde $I=\left[t_{0}, t_{0}+a\right]$, $\|f(t, x)\| \leqslant M$, para todo $(t, x) \in I \times \bar{B}_{b}\left(x_{0}\right)$ e que exista um funcional $V \in$ $C\left(I \times \bar{B}_{b}\left(x_{0}\right) \times \bar{B}_{b}\left(x_{0}\right), \mathbb{R}_{+}\right)$tal que

(a) $V(t, x, y)>0$, para $x \neq y$;

(b) $V(t, x, y)=0$, para $x=y$;

(c) se $\lim _{n \rightarrow \infty} V\left(t, x_{n}, y_{n}\right)=0$, para cada $t \in I$, então $\lim _{n \rightarrow \infty}\left(x_{n}-y_{n}\right)=0$;

(d) $V(t, x, y)$ seja continuamente Fréchet diferenciável $e$

$\frac{\partial V}{\partial t}(t, x, y)+\left(\frac{\partial V}{\partial x}(t, \cdot, y)\right) f(t, x)+\left(\frac{\partial V}{\partial y}(t, x, \cdot)\right) f(t, y) \leqslant 0$

(e) para todo número positivo $K$ as funções $\frac{\partial V}{\partial t}(t, x, y), \frac{\partial V}{\partial x}(t, \cdot, y) u$ e $\frac{\partial V}{\partial y}(t, x, \cdot) u$ sejam contínuas em $(x, y)$ uniformemente para $(t, x, y) \in I \times \bar{B}_{b}\left(x_{0}\right) \times \bar{B}_{b}\left(x_{0}\right)$ e u $\in X, \operatorname{com}\|u\| \leqslant K$.

Então (2.1) possui uma única solução sobre $\left[t_{0}, t_{0}+\alpha\right]$, onde $\alpha \leqslant \min \left\{a, \frac{b}{M}\right\}$. E ainda, a solução depende continuamente do dado inicial $\left(t_{0}, x_{0}\right)$.

Demonstração: Seja $\Delta: t_{0}<t_{1}<\ldots<t_{n}=t_{0}+\alpha$ uma subdivisão de $\left[t_{0}, t_{0}+\alpha\right]$. Definimos a função $\phi_{\Delta}:\left[t_{0}, t_{0}+\alpha\right] \longrightarrow X$ da seguinte forma

$$
\begin{aligned}
& \phi_{\Delta}\left(t_{0}\right)=x_{0} \\
& \phi_{\Delta}(t)=\phi_{\Delta}\left(t_{k-1}\right)+\int_{t_{k-1}}^{t} f\left(s, \phi_{\Delta}\left(t_{k-1}\right)\right) d s, \quad t_{k-1}<t \leqslant t_{k}, \quad k=1,2, \ldots, n .
\end{aligned}
$$

Claramente, desta definição, segue que $\phi_{\Delta}(t)$ está bem definida sobre $\left[t_{0}, t_{0}+\alpha\right]$ e

$$
\phi_{\Delta}\left(t_{k-1}\right)=\phi_{\Delta}\left(t_{k-2}\right)+\int_{t_{k-2}}^{t_{k-1}} f\left(s, \phi_{\Delta}\left(t_{k-2}\right)\right) d s, \quad k=2,3, \ldots, n .
$$

Daí

$$
\phi_{\Delta}(t)=\phi_{\Delta}\left(t_{k-2}\right)+\int_{t_{k-2}}^{t_{k-1}} f\left(s, \phi_{\Delta}\left(t_{k-2}\right)\right) d s+\int_{t_{k-1}}^{t} f\left(s, \phi_{\Delta}\left(t_{k-1}\right)\right) d s
$$


$=\phi_{\Delta}\left(t_{0}\right)+\int_{t_{0}}^{t_{1}} f\left(s, \phi_{\Delta}\left(t_{0}\right)\right) d s+\ldots+\int_{t_{k-2}}^{t_{k-1}} f\left(s, \phi_{\Delta}\left(t_{k-2}\right)\right) d s+\int_{t_{k-1}}^{t} f\left(s, \phi_{\Delta}\left(t_{k-1}\right)\right) d s$.

Definimos, então,

$$
\phi_{\Delta}(t) \equiv x_{0}+\int_{t_{0}}^{t} f_{\Delta}(s) d s
$$

onde a função $f_{\Delta}:\left[t_{0}, t_{0}+\alpha\right] \longrightarrow X$ é dada por $f_{\Delta}(s)=f\left(s, \phi\left(t_{k}\right)\right)$, com $t_{k}<s \leqslant t_{k+1}$ e $\phi\left(t_{k}\right)=\phi_{\Delta}\left(t_{k-1}\right)$.

Notemos que

$$
\phi_{\Delta}^{\prime}(t)=f\left(t, \phi_{\Delta}\left(t_{k-1}\right)\right), \quad t_{k-1}<t \leqslant t_{k} .
$$

De (2.42) e da hipótese, temos

$$
\left\|\phi_{\Delta}(t)-x_{0}\right\| \leqslant \int_{t_{0}}^{t}\left\|f_{\Delta}(s)\right\| d s \leqslant M \alpha \leqslant b, \quad \text { com } t_{0} \leqslant t \leqslant t_{0}+\alpha .
$$

E portanto, $\phi_{\Delta}(t) \in \bar{B}_{b}\left(x_{0}\right)$ para $t_{0} \leqslant t \leqslant t_{0}+\alpha$. Consideremos agora $\Delta$ e $\tilde{\Delta}$ duas subdivisões de $\left[t_{0}, t_{0}+\alpha\right]$ e $\phi_{\Delta}(t), \phi_{\tilde{\Delta}}(t)$ as funções correspondentes. Se $t$ não é um ponto subdivisão de $\Delta$ ou $\tilde{\Delta}, t_{k-1}<t \leqslant t_{k}$ e $\tilde{t}_{j-1}<t \leqslant \tilde{t}_{j}$, então pela Regra da Cadeia para funções Fréchet diferenciáveis, temos

$$
\begin{aligned}
& \frac{d V}{d t}\left(t, \phi_{\Delta}(t), \phi_{\tilde{\Delta}}(t)\right)=\frac{\partial V}{\partial t}+\left(\frac{\partial V}{\partial x}\right) \phi_{\Delta}^{\prime}(t)+\left(\frac{\partial V}{\partial y}\right) \phi_{\tilde{\Delta}}^{\prime}(t) \\
& =\frac{\partial V}{\partial t}+\left(\frac{\partial V}{\partial x}\right) f\left(t, \phi_{\Delta}\left(t_{k-1}\right)\right)+\left(\frac{\partial V}{\partial y}\right) f\left(t, \phi_{\tilde{\Delta}}\left(\tilde{t}_{j-1}\right)\right) .
\end{aligned}
$$

Portanto

$$
\begin{aligned}
& \frac{d V}{d t}\left(t, \phi_{\Delta}(t), \phi_{\tilde{\Delta}}(t)\right)=\left(\frac{\partial V}{\partial t}-\frac{\partial \widetilde{V}}{\partial t}\right)+\frac{\partial \widetilde{V}}{\partial t}+\left[\left(\frac{\partial V}{\partial x}-\frac{\partial \widetilde{V}}{\partial x}\right)+\frac{\partial \widetilde{V}}{\partial x}\right] f\left(t, \phi_{\Delta}\left(t_{k-1}\right)\right) \\
& +\left[\left(\frac{\partial V}{\partial y}-\frac{\partial \widetilde{V}}{\partial y}\right)+\frac{\partial \widetilde{V}}{\partial y}\right] f\left(t, \phi_{\tilde{\Delta}}\left(\tilde{t}_{j-1}\right)\right),
\end{aligned}
$$

onde

$$
\begin{array}{lll}
\frac{\partial V}{\partial t}=\frac{\partial V}{\partial t}\left(t, \phi_{\Delta}(t), \phi_{\tilde{\Delta}}(t)\right) & \frac{\partial \widetilde{V}}{\partial t}=\frac{\partial V}{\partial t}\left(t, \phi_{\Delta}\left(t_{k-1}\right), \phi_{\tilde{\Delta}}\left(\tilde{t}_{j-1}\right)\right) \\
\frac{\partial V}{\partial x}=\frac{\partial V}{\partial x}\left(t, \cdot \phi_{\tilde{\Delta}}(t)\right) & \frac{\partial \widetilde{V}}{\partial x}=\frac{\partial V}{\partial x}\left(t, \cdot \phi_{\tilde{\Delta}}\left(\tilde{t}_{j-1}\right)\right) \\
\frac{\partial V}{\partial y}=\frac{\partial V}{\partial y}\left(t, \phi_{\Delta}(t), \cdot\right) & \frac{\partial \widetilde{V}}{\partial y}=\frac{\partial V}{\partial y}\left(t, \phi_{\Delta}\left(t_{k-1}\right), \cdot\right) .
\end{array}
$$

Pela hipótese $(e)$, segue que para qualquer $\epsilon>0$, existe $\delta>0$ tal que tomando $|\Delta|=\max _{k}\left(t_{k}-t_{k-1}\right)<\delta$ e $|\tilde{\Delta}|=\max _{j}\left(\tilde{t}_{j}-\tilde{t}_{j-1}\right)<\delta$, temos

$$
\begin{aligned}
& \frac{\partial V}{\partial t}-\frac{\partial \widetilde{V}}{\partial t}<\frac{\epsilon}{3} \\
& \left(\frac{\partial V}{\partial x}-\frac{\partial \tilde{V}}{\partial x}\right) f\left(t, \phi_{\Delta}\left(t_{k-1}\right)\right)<\frac{\epsilon}{3}, \quad \operatorname{com}\left\|f\left(t, \phi_{\Delta}\left(t_{k-1}\right)\right)\right\| \leqslant M \\
& \left(\frac{\partial V}{\partial y}-\frac{\partial \tilde{V}}{\partial y}\right) f\left(t, \phi_{\tilde{\Delta}}\left(\tilde{t}_{j-1}\right)\right)<\frac{\epsilon}{3}, \quad \operatorname{com}\left\|f\left(t, \phi_{\tilde{\Delta}}\left(\tilde{t}_{j-1}\right)\right)\right\| \leqslant M .
\end{aligned}
$$


De (2.43), (2.44) e da hipótese $(d)$, obtemos

$$
\frac{d V}{d t}\left(t, \phi_{\Delta}(t), \phi_{\tilde{\Delta}}(t)\right)<\epsilon .
$$

Integrando de $t_{0}$ a $t$, temos

$$
V\left(t, \phi_{\Delta}(t), \phi_{\bar{\Delta}}(t)\right)=V\left(t, \phi_{\Delta}(t), \phi_{\bar{\Delta}}(t)\right)-V\left(t_{0}, x_{0}, x_{0}\right)<\epsilon\left(t-t_{0}\right) \leqslant \epsilon \alpha .
$$

A estimativa (2.45), juntamente com as hipóteses $(a)$ e $(b)$, implicam que as $\phi_{\Delta}$ 's formam uma seqüência de Cauchy. Uma vez que $X$ é um espaço completo, então existe uma função $x(t)$ tal que

$$
\lim _{|\Delta| \rightarrow 0} \phi_{\Delta}(t)=x(t), \quad t \in\left[t_{0}, t_{0}+\alpha\right]
$$

Como $\phi_{\Delta}(t) \in \bar{B}_{b} \subset X$ e $\bar{B}_{b}$ é fechado, logo $x(t) \in \bar{B}_{b}$. Agora, fixamos $t \in$ $\left[t_{0}, t_{0}+\alpha\right]$. Uma vez que para cada subdivisão $\Delta$ existe um $k$ tal que $t_{k-1}<t \leqslant t_{k}$ e

$$
\left\|\phi_{\Delta}\left(t_{k-1}\right)-\phi_{\Delta}(t)\right\|=\left\|\int_{t_{0}}^{t_{k-1}} f_{\Delta}(s) d s-\int_{t_{0}}^{t} f_{\Delta}(s) d s\right\| \leqslant M\left(t-t_{k-1}\right) \leqslant M|\Delta| .
$$

Então

$$
\lim _{|\Delta| \rightarrow 0} \phi_{\Delta}\left(t_{k-1}\right)=x(t)
$$

Logo

$$
f\left(t, \phi_{\Delta}\left(t_{k-1}\right)\right)=f_{\Delta}(t) \rightarrow f(t, x(t)), \quad \text { quando }|\Delta| \rightarrow 0 .
$$

Pelo Teorema da Convergência Dominada (ver [31], pág. 579, Teorema D.8.4) e por (2.42), obtemos

$$
x(t)=x_{0}+\int_{t_{0}}^{t} f(s, x(s)) d s .
$$

Portanto a parte da existência está provada. Provaremos agora a unicidade. Para isto, assumimos que $x_{1}(t)$ e $x_{2}(t)$ são duas soluções de (2.1). Então pela hipótese $(d)$,

$$
\frac{d V}{d t}\left(t, x_{1}(t), x_{2}(t)\right)=\frac{\partial V}{\partial t}+\left(\frac{\partial V}{\partial x}\right) f\left(t, x_{1}(t)\right)+\left(\frac{\partial V}{\partial y}\right) f\left(t, x_{2}(t)\right) \leqslant 0
$$

e, integrando de $t_{0}$ a $t$, obtemos

$$
V\left(t, x_{1}(t), x_{2}(t)\right)=V\left(t, x_{1}(t), x_{2}(t)\right)-V\left(t_{0}, x_{0}, x_{0}\right) \leqslant 0
$$

De $(a)$ e $(b)$, temos $x_{1}(t)=x_{2}(t)$. 
Para finalizarmos, provaremos a dependência contínua das soluções em relação às condições iniciais. Sejam $x_{1}(t)=x\left(t ; t_{1}, x_{1}\right)$ e $x_{2}(t)=x\left(t ; t_{2}, x_{2}\right)$ soluções de $x^{\prime}=f(t, x)$ através de $\left(t_{1}, x_{1}\right)$ e $\left(t_{2}, x_{2}\right)$, respectivamente. Com base em $(d)$, obtemos $\frac{d V}{d t}\left(t, x_{1}(t), x_{2}(t)\right)=\frac{\partial V}{\partial t}+\left(\frac{\partial V}{\partial x}\right) f\left(t, x\left(t ; t_{1}, x_{1}\right)\right)+\left(\frac{\partial V}{\partial y}\right) f\left(t, x\left(t ; t_{2}, x_{2}\right)\right) \leqslant 0$.

Integrando de $t_{1}$ a $t$, temos

$$
0 \leqslant V\left(t, x_{1}(t), x_{2}(t)\right) \leqslant V\left(t_{1}, x_{1}, x_{2}\left(t_{1}\right)\right) .
$$

Suponhamos agora que $\left(t_{1}, x_{1}\right) \rightarrow\left(t_{2}, x_{2}\right)$. Uma vez que $V$ e $x_{2}$ são contínuas, então

$$
V\left(t_{1}, x_{1}, x_{2}\left(t_{1}\right)\right) \rightarrow V\left(t_{2}, x_{2}, x_{2}\left(t_{2}\right)\right)=V\left(t_{2}, x_{2}, x_{2}\right)=0 .
$$

Tomando agora o limite em ambos os lados de (2.46), quando $\left(t_{1}, x_{1}\right) \rightarrow\left(t_{2}, x_{2}\right)$, temos

$$
\lim _{\left(t_{1}, x_{1}\right) \rightarrow\left(t_{2}, x_{2}\right)} V\left(t, x_{1}(t), x_{2}(t)\right)=0 .
$$

De $(c)$, segue que $x\left(t ; t_{1}, x_{1}\right) \rightarrow x\left(t ; t_{2}, x_{2}\right)$ quando $\left(t_{1}, x_{1}\right) \rightarrow\left(t_{2}, x_{2}\right)$.

Corolário 2.3.2 Sejam $H$ um espaço de Hilbert, $f \in C\left(I \times \bar{B}_{b}, H\right)$ e $\|f(t, x)\| \leqslant M$, para $(t, x) \in I \times \bar{B}_{b}$. Suponhamos que $-f$ seja uma função monótona, isto é, existe uma constante positiva $N$ tal que

$$
\Re[\langle f(t, x)-f(t, y), x-y\rangle] \leqslant N\|x-y\|^{2}, \quad t_{0} \leqslant t \leqslant t_{0}+\alpha, \quad x, y \in H,
$$

onde $\Re[\cdot]$ denota a parte real de um número complexo. Então a conclusão do Teorema 2.3.1 é válida.

Demonstração: Para demonstrarmos o corolário, basta exibirmos um funcional $V(t, x, y)$ que satisfaz as hipóteses de $(a)-(e)$ do Teorema 2.3.1. Tomemos então

$$
V(t, x, y)=\exp (-2 N t)\|x-y\|^{2}, \quad \text { onde }\|x\|^{2}=\langle x, x\rangle .
$$

Claramente as hipóteses de $(a)-(c)$ são satisfeitas. Verifiquemos então $(d)$ e $(e)$.

(d) Como $-f$ é monótona obtemos

$$
\begin{aligned}
& \frac{\partial V}{\partial t}(t, x, y)+\left(\frac{\partial V}{\partial x}(t, \cdot, y)\right) f(t, x)+\left(\frac{\partial V}{\partial y}(t, x, \cdot)\right) f(t, y) \\
& =\exp (-2 N t)\left[\langle f(t, x)-f(t, y), x-y\rangle+\langle x-y, f(t, x)-f(t, y)\rangle-2 N\|x-y\|^{2}\right] \\
& =2 \exp (-2 N t)\left[\Re[\langle f(t, x)-f(t, y), x-y\rangle]-N\|x-y\|^{2}\right] \\
& \leqslant 2 \exp (-2 N t)\left[N\|x-y\|^{2}-N\|x-y\|^{2}\right]=0 .
\end{aligned}
$$


(e) Claramente é satisfeita, uma vez que $\frac{\partial V}{\partial t},\left(\frac{\partial V}{\partial x}\right) u$ e $\left(\frac{\partial V}{\partial y}\right) u$, com $\|u\| \leqslant K$, são compostas de funções contínuas.

Assim, o problema de valor inicial (2.1) possui uma única solução sobre $\left[t_{0}, t_{0}+\alpha\right]$, onde $\alpha \leqslant \min \left\{a, \frac{b}{M}\right\}$, e a solução depende continuamente de $\left(t_{0}, x_{0}\right)$.

Observação: As condições do Teorema 2.3.1, em especial a condição $(d)$, são mais gerais do que a condição de monotonicidade (2.47) e, algumas vezes, são chamadas condições do tipo dissipativa.

Exemplos: Consideremos $X=\mathbb{R}$ e a função

$$
f(t, x)= \begin{cases}1-\sqrt{x}, & \text { se } x \geqslant 0 \\ 1, & \text { se } x<0 .\end{cases}
$$

A função $f \in C\left(I \times \bar{B}_{b}\left(x_{0}\right), \mathbb{R}\right)$ e $|f(t, x)|$ é limitada para todo $(t, x) \in I \times \bar{B}_{b}\left(x_{0}\right)$. De fato, se $x<0$, então $|f(t, x)|=1$ e se $x \geqslant 0$, então $|f(t, x)| \leqslant 1+\sqrt{b+\left|x_{0}\right|}$.

Notemos que $f$ não satisfaz a condição de Lipschitz, logo não podemos aplicar o Teorema 2.2.1. Entretanto satisfaz a condição de monotonicidade (2.47), com $N=0$. De fato, se $x, y<0$, então isso é imediato. Agora, se $x \geqslant 0$ e $y<0$, temos

$$
\Re[\langle f(t, x)-f(t, y), x-y\rangle]=(1-\sqrt{x}-1)(x-y)=-x \sqrt{x}+y \sqrt{x} \leqslant 0 .
$$

E se $x, y \geqslant 0$, então

$$
(\sqrt{y}-\sqrt{x})(x-y)=(\sqrt{y}-\sqrt{x})(\sqrt{x}-\sqrt{y})(\sqrt{x}+\sqrt{y})=-(\sqrt{x}-\sqrt{y})^{2}(\sqrt{x}+\sqrt{y}) \leqslant 0 .
$$

Portanto, podemos aplicar o Corolário 2.3.2.

Por outro lado, a função

$$
f(t, x)= \begin{cases}1+\sqrt{x}, & \text { se } x \geqslant 0 \\ 1, & \text { se } x<0\end{cases}
$$

não satisfaz a condição de monotonicidade (2.47). Para verificarmos isto, suponhamos, por contradição, que (2.47) seja satisfeita. Logo existe uma constante positiva $N$ tal que para todo $x, y \in X$,

$$
(\sqrt{x}-\sqrt{y})(x-y) \leqslant N(x-y)^{2} .
$$


Em particular, tomando $x=\frac{1}{N^{3}}$ e $y=0$ chegaremos em um absurdo.

Entretanto, existe um funcional $V(t, x, y)$ satisfazendo as hipóteses do Teorema 2.3.1. Este é dado por

$$
V(t, x, y)= \begin{cases}{[\sqrt{x}-\sqrt{y}-\log (1+\sqrt{x})+\log (1+\sqrt{y})]^{2},} & \text { se } x \geqslant 0, y \geqslant 0 \\ {\left[\sqrt{x}-\log (1+\sqrt{x})+\frac{1}{2} y\right]^{2},} & \text { se } x \geqslant 0, y<0 \\ {\left[\frac{1}{2} x-\sqrt{y}+\log (1+\sqrt{y})\right]^{2},} & \text { se } x<0, y \geqslant 0 \\ {\left[\frac{1}{4}(x-y)^{2}\right]^{2},} & \text { se } x<0, y<0 .\end{cases}
$$

A seguir, veremos um teorema mais geral de unicidade em um espaço normado $X$. O mesmo também pode ser aplicado em problemas envolvendo Equações Diferenciais Parciais. Inicialmente consideremos $I=\left[t_{0}, t_{0}+a\right]$, e para cada $t \in I$ seja $D(t)$ um subconjunto de $X$. Definimos $D=\{(t, x): \quad t \in I$ e $x \in D(t)\}$ e consideremos o problema de valor inicial (2.1), com $x\left(t_{0}\right)=x_{0} \in D\left(t_{0}\right)$ e $f: D \longrightarrow X$ não necessariamente contínua.

Teorema 2.3.3 Suponhamos que exista um funcional $V \in C\left(I \times D \times D, \mathbb{R}_{+}\right)$satisfazendo as hipóteses $(a),(b) e(d)$ do Teorema 2.3.1. Então (2.1) tem no máximo uma solução. E ainda, se a hipótese (c) for satisfeita, então a solução depende continuamente da condição inicial.

Demonstração: A demonstração é idêntica à prova da unicidade e dependência contínua do Teorema 2.3.1.

No exemplo a seguir, aplicaremos o Teorema 2.3.3 para provar um resultado de unicidade de soluções para equação diferencial parcial parabólica.

Exemplo: Consideremos a equação parabólica

$$
u_{t}=u_{x x}+F(t, x, u)
$$

sobre a região limitada por $t=t_{0}, t=t_{0}+a, x=\lambda_{1}(t)$ e $x=\lambda_{2}(t)$, onde $\lambda_{1}(t)$ e $\lambda_{2}(t)$ são diferenciáveis sobre $I$ e $\lambda_{1}(t)<\lambda_{2}(t)$ para $t \in I$. Tomemos as seguintes condições iniciais e de fronteira: $u=g(x)$ sobre $t=t_{0}, u=h_{1}(t)$ sobre $x=\lambda_{1}(t)$ e $u=h_{2}(t)$ sobre $x=\lambda_{2}(t)$, onde $g, h_{1}$ e $h_{2}$ são contínuas e $g\left(\lambda_{1}\left(t_{0}\right)\right)=h_{1}\left(t_{0}\right)$ e $g\left(\lambda_{2}\left(t_{0}\right)\right)=h_{2}\left(t_{0}\right)$. 
Suponhamos que para uma constante $k, F\left(t, x, u_{1}\right)-F\left(t, x, u_{2}\right) \leqslant k\left(u_{1}-u_{2}\right)$, para $u_{1}>u_{2}$. Então o problema (2.48) tem no máximo uma solução. De fato, tomemos $X=L^{2}(\mathbb{R})$ como sendo o espaço de Banach das funções $u: \mathbb{R} \longrightarrow \mathbb{R}$ mensuráveis (ver Definição A.1.3) tais que $\|u\|_{L^{2}(\mathbb{R})}^{2}<\infty$, onde

$$
\|u\|_{L^{2}(\mathbb{R})}=\left(\int_{\mathbb{R}}|u(x)|^{2} d x\right)^{\frac{1}{2}} .
$$

Para cada $t \in I$ definimos $D(t)$ como sendo o espaço de todas as funções $u=$ $u(x) \in X$, que são contínuas sobre $\left[\lambda_{1}(t), \lambda_{2}(t)\right]$, pertencem a $C^{2}$ sobre $] \lambda_{1}(t), \lambda_{2}(t)[$, são nulas fora de $\left[\lambda_{1}(t), \lambda_{2}(t)\right]$ e assumem os valores $h_{1}(t)$ e $h_{2}(t)$ em $x=\lambda_{1}(t)$ e $x=\lambda_{2}(t)$, respectivamente. Para $(t, u) \in I \times D(t)$, definimos

$$
f(t, u)=u_{x x}+F(t, x, u) .
$$

Então (2.48), juntamente com as condições iniciais e de fronteira, é equivalente ao problema de valor inicial

$$
\begin{aligned}
& \frac{d u}{d t}=f(t, u) \\
& u\left(t_{0}\right)=g .
\end{aligned}
$$

Definimos

$$
V(t, u, v)=\exp (-2 k t) \int_{\lambda_{1}(t)}^{\lambda_{2}(t)}|u(x)-v(x)|^{2} d x .
$$

Verifiquemos que as condições do Teorema 2.3 .3 são satisfeitas para o problema (2.49) com o funcional $V$ dado por (2.50). Notemos que as hipóteses de $(a)-(c)$ são imediatas. Mostremos então que $(d)$ é satisfeita. Temos

$$
\begin{aligned}
& \frac{\partial V}{\partial t}+\left(\frac{\partial V}{\partial u}\right) f(t, u)+\left(\frac{\partial V}{\partial v}\right) f(t, v)=-2 k \exp (-2 k t) \int_{\lambda_{1}(t)}^{\lambda_{2}(t)}|u(x)-v(x)|^{2} d x \\
& +\exp (-2 k t)\left(\left|u\left(\lambda_{2}(t)\right)-v\left(\lambda_{2}(t)\right)\right|^{2} \lambda_{2}^{\prime}(t)-\left|u\left(\lambda_{1}(t)\right)-v\left(\lambda_{1}(t)\right)\right|^{2} \lambda_{1}^{\prime}(t)\right) \\
& +\exp (-2 k t) \int_{\lambda_{1}(t)}^{\lambda_{2}(t)} 2(u(x)-v(x)) f(t, u) d x-\exp (-2 k t) \int_{\lambda_{1}(t)}^{\lambda_{2}(t)} 2(u(x)-v(x)) f(t, v) d x \\
& =J_{1}+J_{2}+J_{3}+J_{4} .
\end{aligned}
$$


Uma vez que $u\left(\lambda_{i}(t)\right)=v\left(\lambda_{i}(t)\right)=h_{i}(t)$ para $i=1,2$, então $J_{2}=0$. Da definição de $f(t, u)$, obtemos

$$
\begin{aligned}
& J_{3}+J_{4}=2 \exp (-2 k t) \int_{\lambda_{1}(t)}^{\lambda_{2}(t)}(u(x)-v(x))(f(t, u)-f(t, v)) d x \\
& =2 \exp (-2 k t) \int_{\lambda_{1}(t)}^{\lambda_{2}(t)}(u(x)-v(x))\left(u_{x x}+F(t, x, u)-v_{x x}-F(t, x, v)\right) d x=2 \exp (-2 k t) \\
& {\left[\int_{\lambda_{1}(t)}^{\lambda_{2}(t)}(u(x)-v(x))(F(t, x, u)-F(t, x, v)) d x+\int_{\lambda_{1}(t)}^{\lambda_{2}(t)}(u(x)-v(x))\left(u_{x x}-v_{x x}\right) d x\right] .}
\end{aligned}
$$

Utilizando integração por partes, temos

$$
\begin{aligned}
& \int_{\lambda_{1}(t)}^{\lambda_{2}(t)}(u(x)-v(x))\left(u_{x x}-v_{x x}\right) d x=\left.(u(x)-v(x))\left(u_{x}-v_{x}\right)\right|_{\lambda_{1}(t)} ^{\lambda_{2}(t)} \\
- & \int_{\lambda_{1}(t)}^{\lambda_{2}(t)}\left(u_{x}-v_{x}\right)\left(u_{x}-v_{x}\right) d x .
\end{aligned}
$$

Daí,

$$
\begin{aligned}
J_{3}+J_{4} & =2 \exp (-2 k t) \int_{\lambda_{1}(t)}^{\lambda_{2}(t)}(u(x)-v(x))(F(t, x, u)-F(t, x, v)) d x \\
& -2 \exp (-2 k t) \int_{\lambda_{1}(t)}^{\lambda_{2}(t)}\left|u_{x}-v_{x}\right|^{2} d x \\
& \leqslant 2 \exp (-2 k t) \int_{\lambda_{1}(t)}^{\lambda_{2}(t)}(u(x)-v(x))(F(t, x, u)-F(t, x, v)) d x \\
& \leqslant 2 k \exp (-2 k t) \int_{\lambda_{1}(t)}^{\lambda_{2}(t)}(u(x)-v(x))^{2} d x .
\end{aligned}
$$

Portanto

$$
\frac{\partial V}{\partial t}+\left(\frac{\partial V}{\partial u}\right) f(t, u)+\left(\frac{\partial V}{\partial v}\right) f(t, v) \leqslant 0
$$

Assim, pelo Teorema 2.3.3, o problema de valor inicial (2.49) tem no máximo uma solução. Conseqüentemente (2.48) tem no máximo uma solução. 


\subsection{Diferenciabilidade em Relação às Condições}

\section{Iniciais}

Consideremos o problema de Cauchy não linear (2.1), com $f: \mathbb{R}_{+} \times X \longrightarrow X$. Estudamos, na seção anterior, a existência local e unicidade de soluções para o problema (2.1) e sua dependência contínua em relação às condições iniciais $\left(t_{0}, x_{0}\right)$. Veremos, nesta seção, a diferenciabilidade em relação às condições iniciais e mostraremos que as derivadas de Fréchet da solução $x\left(t ; t_{0}, x_{0}\right)$ de $(2.1)$, em relação aos valores iniciais $\left(t_{0}, x_{0}\right)$, existem e juntamente com $x\left(t ; t_{0}, x_{0}\right)$ satisfazem a equação variacional (que definiremos depois) de (2.1). Para tanto, iremos supor que existe a derivada de Fréchet de $f$ em relação à segunda variável $x, d_{x} f(t, \cdot)$, e precisaremos de alguns resultados, que serão trabalhados a seguir.

Definição 2.4.1 Um funcional contínuo (não linear) $\Phi: X \longrightarrow \mathbb{R}_{+}$é admissível em $X$, se as seguintes condições são satisfeitas:

(i) $\Phi(x)>0, \quad$ para $\quad x \in X, \quad x \neq 0 \quad$ e $\Phi(0)=0$;

(ii) se $\lim _{n \rightarrow \infty} \Phi\left(x_{n}\right)=0$, para $x_{n} \in X$, então $\lim _{n \rightarrow \infty} x_{n}=0$;

(iii) existe uma aplicação $M: X \times X \longrightarrow \mathbb{R}$ tal que $M[x, h]$ é contínua em $h$, uniformemente em relação à $x$ em qualquer bola fechada $\bar{B}_{r}\left(x_{0}\right)$, satisfazendo as propriedades :

(a) $\Phi(x+h)-\Phi(x) \leqslant M[x, h]+O(\|h\|), \quad$ para $\quad x, h \in X$;

(b) $M[x, \lambda h]=\lambda M[x, h], \quad$ para $\lambda \geqslant 0 \quad$ e $\quad x, h \in X$;

(c) $M\left[x, h_{1}+h_{2}\right] \leqslant M\left[x, h_{1}\right]+M\left[x, h_{2}\right]$, para $x, h_{1}, h_{2} \in X$.

\section{Exemplos:}

1. O funcional $\Phi(x)=\|x\|$ é admissível em qualquer espaço de Banach $X$ com $M[x, h]=\|h\|$.

2. O funcional $\Phi(x)=\langle x, x\rangle$ é admissível em qualquer espaço de Hilbert $H$ com $M[x, h]=2 \Re[\langle x, h\rangle]$. 
A prova dos exemplos acima é uma conseqüência imediata das propriedades de norma e produto interno.

Provaremos, a seguir, que o funcional $M[x, \cdot]$ é limitado.

Lema 2.4.2 Seja $\Phi$ um funcional admissível em X. Então, para qualquer bola fechada $\bar{B}_{r}\left(x_{0}\right)$, existe uma constante $k(r)$ tal que

$$
|M[x, h]|<k(r)\|h\|, \quad \text { para } \quad x \in \bar{B}_{r}, \quad h \in X .
$$

Demonstração: Como $M[x, h]$ é contínua em $h$, uniformemente em relação à $x \in \bar{B}_{r}$ e como

$$
M[x, 0]=M[x, 0 h]=0 M[x, h]=0 \Longrightarrow M[x, 0]=0,
$$

então, dado $\epsilon>0$, existe um $\delta=\delta(\epsilon, r)>0$ tal que, se $\|\tilde{h}-0\| \leqslant \delta$ então $|M[x, \tilde{h}]-M[x, 0]|<\epsilon$ para todo $x \in \bar{B}_{r}$, isto é,

$$
\|\tilde{h}\| \leqslant \delta \Longrightarrow|M[x, \tilde{h}]|<\epsilon, \quad \forall x \in \bar{B}_{r}
$$

Para um $h$ arbitrário consideremos $\tilde{h}=\frac{\delta}{\|h\|} h$. Então $\|\tilde{h}\|=\delta$ e assim

$$
|M[x, \tilde{h}]|<\epsilon \Longrightarrow\left|M\left[x, \frac{\delta}{\|h\|} h\right]\right|<\epsilon, \quad x \in \bar{B}_{r}, \quad h \in X .
$$

Uma vez que $M\left[x, \frac{\delta}{\|h\|} h\right]=\frac{\delta}{\|h\|} M[x, h]$, temos

$$
|M[x, h]|<\frac{\epsilon}{\delta}\|h\|, \quad x \in \bar{B}_{r}, \quad h \in X .
$$

Tomando, agora, $k(r)=\frac{\epsilon}{\delta}$, obtemos

$$
|M[x, h]|<k(r)\|h\|, \quad x \in \bar{B}_{r}, \quad h \in X
$$

Consideremos, agora, o problema de valor inicial (2.1), com $f: \mathbb{R}_{+} \times X \longrightarrow X$, e o problema de valor inicial escalar

$$
\begin{aligned}
& r^{\prime}=g(t, r) \\
& r\left(t_{0}\right)=r_{0},
\end{aligned}
$$

onde $g: \mathbb{R}_{+} \times \mathbb{R}_{+} \longrightarrow \mathbb{R}$. 
Assumiremos que $f$ e $g$ satisfazem condições suficientes para garantir a existência (não unicidade) de soluções dos problemas (2.1) e (2.51) para todo $t \in \mathbb{R}_{+}$. Efetivamente, existência local bastaria e nossos resultados poderiam ser reafirmados para valerem localmente. É claro que, como já vimos, somente a continuidade de $f$ não é suficiente nem mesmo para assegurar a existência local de soluções de (2.1).

Denotaremos uma solução de (2.1) por $x\left(t ; t_{0}, x_{0}\right)$ e a solução maximal de (2.51) por $r\left(t ; t_{0}, r_{0}\right)$. Assumiremos também a existência de um funcional admissível $\Phi$ em $X$ satisfazendo as propriedades $(i)$ - (iii) da Definição 2.4.1.

Consideremos as seguintes hipóteses:

Hipótese 1: $M[x-y, f(t, x)-f(t, y)] \leqslant g(t, \Phi(x-y))$, para $t \in \mathbb{R}_{+}$e $\forall x, y \in X$;

Hipótese 2: A função $f(t, x)$ é continuamente Fréchet diferenciável em relação à variável $x \mathrm{e}$

$$
M\left[h, d_{x} f(t, z) h\right] \leqslant g(t, \Phi(h)), \quad t \in \mathbb{R}_{+}, \quad h \in X,
$$

e todo $z$ em qualquer bola fechada $\bar{B}_{r}\left(x_{0}\right)$.

Provaremos, mais adiante, que a Hipótese 2 implica a Hipótese 1. Inicialmente, devemos mostrar que se a Hipótese 1 for satisfeita, então o problema (2.1) possui uma única solução $x\left(t ; t_{0}, x_{0}\right)$, que depende continuamente das condições iniciais $\left(t_{0}, x_{0}\right)$, descle que o problema de valor inicial escalar (2.51) possua estas propriedades.

O símbolo $D_{+} r(t)$ no lema a seguir denota a derivada de Dini inferior à direita da função $r(t)$, isto é,

$$
D_{+} r(t)=\lim _{h \rightarrow 0^{+}} \inf \frac{r(t+h)-r(t)}{h} .
$$

Para mais detalhes sobre derivadas de Dini, ver [39], pág. 239.

Lema 2.4.3 Para qualquer função diferenciável $x: \mathbb{R}_{+} \longrightarrow X$, a seguinte desigualdade é satisfeita

$$
D_{+} \Phi(x(t)) \leqslant M\left[x(t), x^{\prime}(t)\right], \quad t \in \mathbb{R}_{+} .
$$

Demonstração:

$$
D_{+} \Phi(x(t))=\lim _{h \rightarrow 0^{+}} \inf \frac{\Phi(x(t+h))-\Phi(x(t))}{h}
$$




$$
\begin{aligned}
& =\lim _{h \rightarrow 0^{+}} \inf \frac{\Phi\left(x(t)+x^{\prime}(t) h+O\left(h^{2}\right)\right)-\Phi(x(t))}{h} \\
& \leqslant \lim _{h \rightarrow 0^{+}} \inf \left[\frac{M\left[x(t), x^{\prime}(t) h+O\left(h^{2}\right)\right]}{h}+O\left(\left\|x^{\prime}(t) h+O\left(h^{2}\right)\right\|\right)\right] \\
& \leqslant \lim _{h \rightarrow 0^{+}} \inf \left[\frac{h M\left[x(t), x^{\prime}(t)\right]}{h}+M\left[x(t), \frac{O\left(h^{2}\right)}{h}\right]\right] \\
& =M\left[x(t), x^{\prime}(t)\right]+\lim _{h \rightarrow 0^{+}} \inf M[x(t), O(h)]=M\left[x(t), x^{\prime}(t)\right] .
\end{aligned}
$$

O lema que veremos a seguir será muito útil em vários resultados desta e da próxima seção. Sua demonstração pode ser encontrada em [21], pág. 26, Teorema 4.1.

Lema 2.4.4 Suponhamos que $g(t, r)$ seja contínua em um aberto $\Omega \subset \mathbb{R}_{+} \times \mathbb{R}_{+} e$ $r(t)$ seja a solução maximal de (2.51). Consideremos $v(t)$ uma função contínua sobre $\left[t_{0}, t_{0}+a\right]$ satisfazendo as seguintes condições: $v\left(t_{0}\right) \leqslant r_{0},(t, v(t)) \in \mathbb{R}_{+} \times \mathbb{R}_{+}$e $v(t)$ possui uma derivada à direita $\frac{d^{+} v(t)}{d t}=\lim _{h \rightarrow 0^{+}} \frac{v(t+h)-v(t)}{h}$ sobre $t_{0} \leqslant t<t_{0}+a$ tal $q u e \frac{d^{+} v(t)}{d t} \leqslant g(t, v(t))$. Então, sobre um intervalo comum de existência de $r(t)$ e $v(t)$ temos que $v(t) \leqslant r(t)$.

O lema a seguir será usado para provarmos a unicidade de soluções de (2.1).

Lema 2.4.5 Suponhamos que a Hipótese 1 seja satisfeita e que $\Phi\left(x_{0}-y_{0}\right) \leqslant r_{0}$. Então

$$
\Phi\left(x\left(t ; t_{0}, x_{0}\right)-y\left(t ; t_{0}, y_{0}\right)\right) \leqslant r\left(t ; t_{0}, r_{0}\right), \quad t \geqslant t_{0} .
$$

Demonstração: Sejam $x(t)=x\left(t ; t_{0}, x_{0}\right)$ e $y(t)=y\left(t ; t_{0}, y_{0}\right)$ as soluções de (2.1) através de $\left(t_{0}, x_{0}\right)$ e $\left(t_{0}, y_{0}\right)$, respectivamente, e $r\left(t ; t_{0}, r_{0}\right)$ a solução maximal de (2.51). Definimos $z(t)=x(t)-y(t)$. Como $z(t)$ é uma função diferenciável, então, pelo Lema 2.4.3,

$$
\begin{aligned}
& D_{+} \Phi(z(t)) \leqslant M\left[z(t), z^{\prime}(t)\right]=M\left[x(t)-y(t), x^{\prime}(t)-y^{\prime}(t)\right] \\
& =M[x(t)-y(t), f(t, x(t))-f(t, y(t))] \leqslant g(t, \Phi(x(t)-y(t)))=g(t, \Phi(z(t))),
\end{aligned}
$$

para $t \geqslant t_{0}$. E ainda,

$$
\Phi\left(z\left(t_{0}\right)\right)=\Phi\left(x\left(t_{0}\right)-y\left(t_{0}\right)\right)=\Phi\left(x_{0}-y_{0}\right) \leqslant r_{0} .
$$

Pelo Lema 2.4.4, temos $\Phi(z(t)) \leqslant r(t)$, isto é,

$$
\Phi\left(x\left(t ; t_{0}, x_{0}\right)-y\left(t ; t_{0}, y_{0}\right)\right) \leqslant r\left(t ; t_{0}, r_{0}\right), \quad t \geqslant t_{0} .
$$


Teorema 2.4.6 Suponhamos que a Hipótese 1 seja satisfeita e que $r\left(t ; t_{0}, 0\right) \equiv 0$. Então o problema (2.1) tem uma única solução.

Demonstração: Sejam $x_{1}(t)=x_{1}\left(t ; t_{0}, x_{0}\right)$ e $x_{2}(t)=x_{2}\left(t ; t_{0}, x_{0}\right)$ duas soluções de (2.1). Como $r\left(t ; t_{0}, 0\right) \equiv 0$ então $r_{0}=0$, e ainda $\Phi\left(x_{0}-x_{0}\right)=\Phi(0)=0=r_{0}$. Uma vez que a Hipótese 1 é satisfeita, segue do Lema 2.4.5 que

$$
\Phi\left(x_{1}(t)-x_{2}(t)\right)=\Phi\left(x_{1}\left(t ; t_{0}, x_{0}\right)-x_{2}\left(t ; t_{0}, x_{0}\right)\right) \leqslant r\left(t ; t_{0}, r_{0}\right) \equiv 0 .
$$

Assim $x_{1}(t)-x_{2}(t)=0$ para $t \geqslant t_{0}$, ou ainda, $x_{1}(t)=x_{2}(t)$ para $t \geqslant t_{0}$.

Observação: Como mencionamos no Exemplo 2, o funcional $\Phi(x)=\langle x, x\rangle$ é admissível em qualquer espaço de Hilbert $H \operatorname{com} M[x, h]=2 \Re[\langle x, h\rangle]$. Neste caso, a Hipótese $1, \operatorname{com} g(t, r)=2 N r$, reduz-se à condição de monotonicidade (2.47) sobre $f$, que usamos para provar existência e unicidade de soluções de (2.1) no Corolário 2.3.2. De fato,

$$
\begin{aligned}
& M[x-y, f(t, x)-f(t, y)] \leqslant g(t, \Phi(x-y)) \\
\Longrightarrow & 2 \Re[\langle x-y, f(t, x)-f(t, y)\rangle] \leqslant 2 N \Phi(x-y)=2 N\langle x-y, x-y\rangle \\
\Longrightarrow & \Re[\langle x-y, f(t, x)-f(t, y)\rangle] \leqslant N\|x-y\|^{2} .
\end{aligned}
$$

Provaremos, no teorema seguinte, a dependência contínua das soluções de (2.1) em relação às condições iniciais.

Teorema 2.4.7 Suponhamos que a Hipótese 1 seja satisfeita e que a solução maximal de (2.51) dependa continuamente de $\left(t_{0}, r_{0}\right)$ para cada $\left(t_{0}, r_{0}\right) \in \mathbb{R}_{+} \times \mathbb{R}_{+} e$, $r\left(t ; t_{0}, 0\right) \equiv 0$ para $t \geqslant t_{0}$. Então, $x\left(t ; t_{0}, x_{0}\right)$ depende continuamente de $\left(t_{0}, x_{0}\right)$.

Demonstração: Sejam $x_{1}(t)=x\left(t ; t_{1}, x_{1}\right)$ e $x_{2}(t)=x\left(t ; t_{2}, x_{2}\right)$ soluções de $x^{\prime}=f(t, x)$ através de $\left(t_{1}, x_{1}\right)$ e $\left(t_{2}, x_{2}\right)$, respectivamente. Consideremos $t_{2} \geqslant t_{1}$ e definimos $z(t)=x_{1}(t)-x_{2}(t)$.

Como $z: \mathbb{R}_{+} \longrightarrow X$ é diferenciável, então, pelo Lema 2.4.3,

$$
\begin{aligned}
& D_{+} \Phi(z(t)) \leqslant M\left[z(t), z^{\prime}(t)\right]=M\left[x_{1}(t)-x_{2}(t), f\left(t, x_{1}(t)\right)-f\left(t, x_{2}(t)\right)\right] \\
& \leqslant g\left(t, \Phi\left(x_{1}(t)-x_{2}(t)\right)\right)=g(t, \Phi(z(t))), \quad t \geqslant t_{1} .
\end{aligned}
$$

E ainda,

$$
\Phi\left(z\left(t_{1}\right)\right)=\Phi\left(x\left(t_{1} ; t_{1}, x_{1}\right)-x\left(t_{1} ; t_{2}, x_{2}\right)\right)=\Phi\left(x_{1}-x\left(t_{1} ; t_{2}, x_{2}\right)\right) .
$$


Logo, pelo Lema $2.4 .4, \Phi(z(t)) \leqslant r\left(t ; t_{1}, \Phi\left(x_{1}-x\left(t_{1} ; t_{2}, x_{2}\right)\right)\right)$.

Uma vez que $\Phi(x)$ é um funcional contínuo em $x, x\left(t ; t_{2}, x_{2}\right)$ é contínua em $t$ e por hipótese $r\left(t ; t_{1}, r_{1}\right)$ é contínua em $\left(t_{1}, r_{1}\right)$, então

$$
\lim _{\left(t_{1}, x_{1}\right) \rightarrow\left(t_{2}, x_{2}\right)} \Phi(z(t)) \leqslant r\left(t ; t_{2}, \Phi\left(x_{2}-x\left(t_{2} ; t_{2}, x_{2}\right)\right)\right)=r\left(t ; t_{2}, 0\right) \equiv 0 .
$$

Daí, pela definição de $\Phi$,

$$
\lim _{\left(t_{1}, x_{1}\right) \rightarrow\left(t_{2}, x_{2}\right)} z(t)=0
$$

ou seja, $x\left(t ; t_{1}, x_{1}\right) \rightarrow x\left(t ; t_{2}, x_{2}\right)$ quando $\left(t_{1}, x_{1}\right) \rightarrow\left(t_{2}, x_{2}\right)$.

Agora devemos provar que, quando a Hipótese 2 for satisfeita e $r\left(t ; t_{0}, 0\right) \equiv 0$, as soluções $x\left(t ; t_{0}, x_{0}\right)$ de $(2.1)$ são continuamente diferenciáveis em relação às condições iniciais $\left(t_{0}, x_{0}\right)$ e as derivadas de Fréchet $\frac{\partial x}{\partial x_{0}}\left(t ; t_{0}, x_{0}\right)$ e $\frac{\partial x}{\partial t_{0}}\left(t ; t_{0}, x_{0}\right)$ existem, e juntamente com a solução $x\left(t ; t_{0}, x_{0}\right)$, satisfazem a equação variacional de (2.1).

Observação: Suponhamos que $d_{x} f(t, x)$ exista e seja contínua. Logo, pela Desigualdade do Valor Médio para funções Fréchet diferenciáveis, a função $f(t, x)$ é localmente Lipschitziana em $x$ e, conseqüentemente, a existência local e unicidade de soluções de (2.1) estão asseguradas. No entanto, se supormos que $f$ seja apenas Lipschitziana em $x$, neste caso $f$ pode não ser Fréchet diferenciável.

Lema 2.4.8 Seja $f \in C\left(\mathbb{R}_{+} \times \bar{B}_{r}\left(x_{0}\right), X\right)$ e suponhamos que $d_{x} f(t, x)$ exista e seja contínua para $x \in \bar{B}_{r}\left(x_{0}\right)$. Então para $x_{1}, x_{2} \in \bar{B}_{r}\left(x_{0}\right)$ e $t \geqslant 0$,

$$
f\left(t, x_{1}\right)-f\left(t, x_{2}\right)=\int_{0}^{1} d_{x} f\left(t, s x_{1}+(1-s) x_{2}\right)\left(x_{1}-x_{2}\right) d s .
$$

Demonstração: Definimos $F(s)=f\left(t, s x_{1}+(1-s) x_{2}\right), \operatorname{com} 0 \leqslant s \leqslant 1$. Como $\bar{B}_{r}\left(x_{0}\right)$ é convexa, então $F(s)$ está bem definida, pois $s x_{1}+(1-s) x_{2} \in \bar{B}_{r}\left(x_{0}\right)$. Pela Regra da Cadeia,

$$
F^{\prime}(s)=d_{x} f\left(t, s x_{1}+(1-s) x_{2}\right)\left(x_{1}-x_{2}\right) .
$$

E ainda,

$$
F(1)-F(0)=\int_{0}^{1} F^{\prime}(s) d s .
$$

Mas $F(1)=f\left(t, x_{1}\right)$ e $F(0)=f\left(t, x_{2}\right)$, assim

$$
f\left(t, x_{1}\right)-f\left(t, x_{2}\right)=\int_{0}^{1} d_{x} f\left(t, s x_{1}+(1-s) x_{2}\right)\left(x_{1}-x_{2}\right) d s
$$


Lema 2.4.9 Suponhamos que a Hipótese 2 seja satisfeita. Então,

$$
M\left[h, \int_{0}^{1} d_{x} f\left(t, s x_{1}+(1-s) x_{2}\right) h d s\right] \leqslant g(t, \Phi(h)), \quad t \geqslant 0, \quad h, x_{1}, x_{2} \in \bar{B}_{r}\left(x_{0}\right) .
$$

Demonstração: Seja $\mathscr{P}: 0=s_{0}<s_{1}<\ldots<s_{n}=1$ qualquer partição de $[0,1]$. Da definição de integral de Riemann para funções contínuas, temos

$$
\int_{0}^{1} d_{x} f\left(t, s x_{1}+(1-s) x_{2}\right) h d s=\lim _{n \rightarrow \infty}\left[\sum_{i=0}^{n-1} d_{x} f\left(t, \tau_{i} x_{1}+\left(1-\tau_{i}\right) x_{2}\right) h \Delta s_{i}\right],
$$

onde $\Delta s_{i}=s_{i+1}-s_{i}$ e $s_{i} \leqslant \tau_{i} \leqslant s_{i+1}$, para $i=0,1, \ldots, n-1$.

Como $M[x, h]$ é contínua em $h$, uniformemente em relação à $x \in \bar{B}_{r}\left(x_{0}\right)$, então

$$
\begin{aligned}
& M\left[h, \int_{0}^{1} d_{x} f\left(t, s x_{1}+(1-s) x_{2}\right) h d s\right] \\
= & M\left[h, \lim _{n \rightarrow \infty}\left[\sum_{i=0}^{n-1} d_{x} f\left(t, \tau_{i} x_{1}+\left(1-\tau_{i}\right) x_{2}\right) h \Delta s_{i}\right]\right] \\
= & \lim _{n \rightarrow \infty} M\left[h, \sum_{i=0}^{n-1} d_{x} f\left(t, \tau_{i} x_{1}+\left(1-\tau_{i}\right) x_{2}\right) h \Delta s_{i}\right] \\
\leqslant & \lim _{n \rightarrow \infty} \sum_{i=0}^{n-1} \Delta s_{i} M\left[h, d_{x} f\left(t, \tau_{i} x_{1}+\left(1-\tau_{i}\right) x_{2}\right) h\right] \leqslant \lim _{n \rightarrow \infty} \sum_{i=0}^{n-1} \Delta s_{i} g(t, \Phi(h)) \\
= & g(t, \Phi(h)) \lim _{n \rightarrow \infty} \sum_{i=0}^{n-1} \Delta s_{i}=g(t, \Phi(h)) . \quad \square
\end{aligned}
$$

Lema 2.4.10 A Hipótese 2 implica a Hipótese 1.

Demonstração: Dos Lemas 2.4 .8 e 2.4.9, obtemos

$$
\begin{aligned}
M[x-y, f(t, x)-f(t, y)] & =M\left[x-y, \int_{0}^{1} d_{x} f(t, s x+(1-s) y)(x-y) d s\right] \\
& \leqslant g(t, \Phi(x-y)) .
\end{aligned}
$$

Corolário 2.4.11 Suponhamos que $f \in C\left(\mathbb{R}_{+} \times \bar{B}_{r}\left(x_{0}\right), X\right)$ e que a Hipótese 2 seja satisfeita. Assumimos que a solução maximal de (2.51) dependa continuamente de $\left(t_{0}, r_{0}\right)$ para cada $\left(t_{0}, r_{0}\right) \in \mathbb{R}_{+} \times \mathbb{R}_{+}$e $r\left(t ; t_{0}, 0\right) \equiv 0$ para $t \geqslant t_{0}$. Então as soluções de (2.1) existem localmente, são únicas e dependem continuamente das condições iniciais. 
Demonstração: Pelo Lema 2.4.10, a Hipótese 2 implica a Hipótese 1, logo o resultado segue dos Teoremas 2.4 .6 e 2.4.7.

Teorema 2.4.12 Suponhamos que $f \in C\left(\mathbb{R}_{+} \times \bar{B}_{r}\left(x_{0}\right), X\right)$ e que a Hipótese 2 seja satisfeita. Assumimos que a solução maximal de $r^{\prime}=g(t, r)$, através de qualquer ponto $\left(t_{0}, 0\right)$, seja identicamente nula para $t \geqslant t_{0}$. Então:

(a) a derivada de Fréchet $\frac{\partial x}{\partial x_{0}}\left(t ; t_{0}, x_{0}\right) \equiv \varphi\left(t ; t_{0}, x_{0}\right)$ existe e satisfaz a equação operador dada a seguir, onde $I_{d}$ é o operador identidade em $X$

$$
\left\{\begin{array}{l}
\varphi^{\prime}=d_{x} f\left(t, x\left(t ; t_{0}, x_{0}\right)\right) \varphi, \quad t \geqslant t_{0} \\
\varphi\left(t_{0}\right)=I_{d}
\end{array}\right.
$$

(b) a derivada de Fréchet $\frac{\partial x}{\partial t_{0}}\left(t ; t_{0}, x_{0}\right) \equiv \psi\left(t ; t_{0}, x_{0}\right)$ existe e satisfaz

$$
\left\{\begin{array}{l}
\psi^{\prime}=d_{x} f\left(t, x\left(t ; t_{0}, x_{0}\right)\right) \psi, \quad t \geqslant t_{0} \\
\psi\left(t_{0}\right)=-f\left(t_{0}, x_{0}\right) .
\end{array}\right.
$$

$E$ ainda, $\psi\left(t ; t_{0}, x_{0}\right)=-\varphi\left(t ; t_{0}, x_{0}\right) f\left(t_{0}, x_{0}\right)$.

As equações em (2.52) e (2.53) são chamadas equações variacionais de (2.1).

\section{Demonstração:}

(a) Uma vez que $d_{x} f(t, \cdot) \in C\left(\mathbb{R}_{+} \times \bar{B}_{r}\left(x_{0}\right), \mathcal{B}(X)\right)$, onde $\mathcal{B}(X)$ é o espaço de Banach de todos os operadores lineares limitados de $X$ nele mesmo, o lado direito de (2.52) é Lipschitz em $\varphi$. Logo, pelo Teorema 2.2.1, a equação (2.52) tem uma única solução, que denotaremos por $\varphi(t)$. Definimos a função

$$
z(t)=x\left(t ; t_{0}, x_{0}+h\right)-x\left(t ; t_{0}, x_{0}\right)-\varphi(t) h, \quad t \geqslant t_{0}, \quad x_{0}, x_{0}+h \in \bar{B}_{r}\left(x_{0}\right) .
$$

Do Lema 2.4.3 temos

$$
\begin{aligned}
& D_{+} \Phi\left[\frac{z(t)}{\|h\|}\right] \leqslant M\left[\frac{z(t)}{\|h\|}, \frac{z^{\prime}(t)}{\|h\|}\right] \\
= & M\left[\frac{z(t)}{\|h\|}, \frac{f\left(t, x\left(t ; t_{0}, x_{0}+h\right)\right)-f\left(t, x\left(t ; t_{0}, x_{0}\right)\right)}{\|h\|}-\frac{d_{x} f\left(t, x\left(t ; t_{0}, x_{0}\right)\right) \varphi(t) h}{\|h\|}\right]
\end{aligned}
$$


Como $f$ é Fréchet diferenciável em relação à variável $x \in \bar{B}_{r}\left(x_{0}\right)$, então

$$
\begin{aligned}
& f\left(t, x\left(t ; t_{0}, x_{0}+h\right)\right)-f\left(t, x\left(t ; t_{0}, x_{0}\right)\right) \\
& =d_{x} f\left(t, x\left(t ; t_{0}, x_{0}\right)\right)\left(x\left(t ; t_{0}, x_{0}+h\right)-x\left(t ; t_{0}, x_{0}\right)\right) \\
& +O\left(\left\|x\left(t ; t_{0}, x_{0}+h\right)-x\left(t ; t_{0}, x_{0}\right)\right\|\right) \\
& =d_{x} f\left(t, x\left(t ; t_{0}, x_{0}\right)\right)(z(t)+\varphi(t) h)+O\left(\left\|x\left(t ; t_{0}, x_{0}+h\right)-x\left(t ; t_{0}, x_{0}\right)\right\|\right) .
\end{aligned}
$$

Consideremos $\omega(h)=O\left(\left\|x\left(t ; t_{0}, x_{0}+h\right)-x\left(t ; t_{0}, x_{0}\right)\right\|\right)$. De (2.54) e (2.55), temos

$$
\begin{aligned}
& D_{+} \Phi\left[\frac{z(t)}{\|h\|}\right] \leqslant M\left[\frac{z(t)}{\|h\|}, \frac{d_{x} f\left(t, x\left(t ; t_{0}, x_{0}\right)\right) z(t)}{\|h\|}+\frac{\omega(h)}{\|h\|}\right] \\
& \leqslant M\left[\frac{z(t)}{\|h\|}, d_{x} f\left(t, x\left(t ; t_{0}, x_{0}\right)\right) \frac{z(t)}{\|h\|}\right]+M\left[\frac{z(t)}{\|h\|}, \frac{\omega(h)}{\|h\|}\right] \\
& \leqslant g\left(t, \Phi\left(\frac{z(t)}{\|h\|}\right)\right)+M\left[\frac{z(t)}{\|h\|}, \frac{\omega(h)}{\|h\|}\right] .
\end{aligned}
$$

Verifiquemos, agora, que em qualquer intervalo compacto contendo $t$,

$$
\lim _{\|h\| \rightarrow 0} M\left[\frac{z(t)}{\|h\|}, \frac{\omega(h)}{\|h\|}\right]=0 .
$$

Definindo $m(t)=\left\|x\left(t ; t_{0}, x_{0}+h\right)-x\left(t ; t_{0}, x_{0}\right)\right\|$, temos

$$
D_{+} m(t)
$$

$=\lim _{h \rightarrow 0^{+}} \inf \frac{\left\|x\left(t+h ; t_{0}, x_{0}+h\right)-x\left(t+h ; t_{0}, x_{0}\right)\right\|-\left\|x\left(t ; t_{0}, x_{0}+h\right)-x\left(t ; t_{0}, x_{0}\right)\right\|}{h}$

$\leqslant \lim _{h \rightarrow 0^{+}} \inf \frac{\left\|\left[x\left(t+h ; t_{0}, x_{0}+h\right)-x\left(t ; t_{0}, x_{0}+h\right)\right]-\left[x\left(t+h ; t_{0}, x_{0}\right)-x\left(t ; t_{0}, x_{0}\right)\right]\right\|}{h}$

$\leqslant\left\|x^{\prime}\left(t ; t_{0}, x_{0}+h\right)-x^{\prime}\left(t ; t_{0}, x_{0}\right)\right\|=\left\|f\left(t, x\left(t ; t_{0}, x_{0}+h\right)\right)-f\left(t, x\left(t ; t_{0}, x_{0}\right)\right)\right\|$.

Do Lema 2.4.8 temos

$$
\begin{aligned}
& D_{+} m(t) \\
\leqslant & \left\|\int_{0}^{1} d_{x} f\left(t, s x\left(t ; t_{0}, x_{0}+h\right)+(1-s) x\left(t ; t_{0}, x_{0}\right)\right)\left(x\left(t ; t_{0}, x_{0}+h\right)-x\left(t ; t_{0}, x_{0}\right)\right) d s\right\| \\
\leqslant & \left\|x\left(t ; t_{0}, x_{0}+h\right)-x\left(t ; t_{0}, x_{0}\right)\right\| \int_{0}^{1}\left\|d_{x} f\left(t, s x\left(t ; t_{0}, x_{0}+h\right)+(1-s) x\left(t ; t_{0}, x_{0}\right)\right)\right\| d s
\end{aligned}
$$




$$
\Longrightarrow D_{+} m(t) \leqslant k_{1} m(t)
$$

onde $k_{1}$ é uma constante tal que $\left\|d_{x} f(t, z)\right\| \leqslant k_{1}$, para $t$ em um intervalo compacto $J$ ao redor de $t_{0}$ e $z$ presente no segmento de linha unindo as soluções $x\left(t ; t_{0}, x_{0}\right)$ e $x\left(t ; t_{0}, x_{0}+h\right)$. Temos também

$$
m\left(t_{0}\right)=\|h\|
$$

Resolvendo o problema (2.57) e (2.58), obtemos

$$
m(t) \leqslant\|h\| \exp k_{1}\left(t-t_{0}\right) \leqslant k_{2}\|h\|, \quad t \in J, \quad \text { (compacto) }
$$

onde $k_{2}$ é uma constante.

Seja $k_{3}$ uma constante tal que

$$
\|\varphi(t)\| \leqslant k_{3}, \quad t \in J .
$$

Da definição de $z(t)$ e usando (2.59) e (2.60), obtemos

$$
\frac{\|z(t)\|}{\|h\|} \leqslant \frac{\left\|x\left(t ; t_{0}, x_{0}+h\right)-x\left(t ; t_{0}, x_{0}\right)\right\|}{\|h\|}+\frac{\|\varphi(t)\|\|h\|}{\|h\|} \leqslant k_{2}+k_{3}=k_{4},
$$

para $t \in J$ e $\|h\|$ suficientemente pequeno. Assim, $\frac{\|z(t)\|}{\|h\|} \in \bar{B}_{k_{4}}(0)$ para $t \in J$ e $\|h\|$ suficientemente pequeno. Logo, pelo Lema 2.4.2,

$$
M\left[\frac{z(t)}{\|h\|}, \frac{\omega(h)}{\|h\|}\right] \leqslant k \frac{\|\omega(h)\|}{\|h\|} .
$$

Finalmente, da definição de $\omega(h)$ e $(2.59)$, temos

$$
\frac{\|\omega(h)\|}{\|h\|} \leqslant \frac{k_{2}\|\omega(h)\|}{m(t)}=\frac{k_{2} O\left(\left\|x\left(t ; t_{0}, x_{0}+h\right)-x\left(t ; t_{0}, x_{0}\right)\right\|\right)}{\left\|x\left(t ; t_{0}, x_{0}+h\right)-x\left(t ; t_{0}, x_{0}\right)\right\|} .
$$

Logo $\frac{\|\omega(h)\|}{\|h\|} \rightarrow 0$ quando $h \rightarrow 0$. Portanto,

$$
\lim _{\|h\| \rightarrow 0} M\left[\frac{z(t)}{\|h\|}, \frac{\omega(h)}{\|h\|}\right]=0 .
$$

Agora, de (2.56), temos

$$
D_{+} \Phi\left(\frac{z(t)}{\|h\|}\right) \leqslant g\left(t, \Phi\left(\frac{z(t)}{\|h\|}\right)\right)+O(1) .
$$

E ainda, $\Phi\left(\frac{z\left(t_{0}\right)}{\|h\|}\right)=\Phi\left(\frac{x_{0}+h-x_{0}-h}{\|h\|}\right)=\Phi(0)=0$. 
Assim, pelo Lema 2.4.4, obtemos

$$
0<\Phi\left(\frac{z(t)}{\|h\|}\right) \leqslant r\left(t ; t_{0}, 0\right) \equiv 0 \Longrightarrow \Phi\left(\frac{z(t)}{\|h\|}\right)=0 .
$$

Daí

$$
\lim _{\|h\| \rightarrow 0} \Phi\left(\frac{z(t)}{\|h\|}\right)=0
$$

Da definição de $\Phi$, temos

$$
\lim _{\|h\| \rightarrow 0} \frac{z(t)}{\|h\|}=0
$$

o que prova que a derivada de Fréchet $\frac{\partial x}{\partial x_{0}}\left(t ; t_{0}, x_{0}\right)$ existe e é igual a $\varphi(t)$. Assim $(a)$ está provado.

(b) Consideramos $\varphi(t)$ sendo como em (2.52) e definimos a função

$$
z(t)=x\left(t ; t_{0}+h, x_{0}\right)-x\left(t ; t_{0}, x_{0}\right)+\varphi(t) f\left(t_{0}, x_{0}\right) h
$$

Então, como em $(a)$,

$$
\begin{aligned}
& D_{+} \Phi\left(\frac{z(t)}{h}\right) \leqslant M\left[\frac{z(t)}{h}, \frac{z^{\prime}(t)}{h}\right] \\
& =M\left[\frac{z(t)}{h}, \frac{f\left(t, x\left(t ; t_{0}+h, x_{0}\right)\right)-f\left(t, x\left(t ; t_{0}, x_{0}\right)\right)}{h}+d_{x} f\left(t, x\left(t ; t_{0}, x_{0}\right)\right) \varphi(t) f\left(t_{0}, x_{0}\right)\right] \\
& =M\left[\frac{z(t)}{h}, \frac{d_{x} f\left(t, x\left(t ; t_{0}, x_{0}\right)\right)\left(z(t)-\varphi(t) f\left(t_{0}, x_{0}\right) h\right)+\omega(h)}{h}\right. \\
& \left.+d_{x} f\left(t, x\left(t ; t_{0}, x_{0}\right)\right) \varphi(t) f\left(t_{0}, x_{0}\right)\right] \\
& \leqslant M\left[\frac{z(t)}{h}, d_{x} f\left(t, x\left(t ; t_{0}, x_{0}\right)\right) \frac{z(t)}{h}\right]+M\left[\frac{z(t)}{h}, \frac{\omega(h)}{h}\right] \leqslant g\left(t, \Phi\left(\frac{z(t)}{h}\right)\right)+O(1),
\end{aligned}
$$

quando $h \rightarrow 0$. E ainda

$$
\begin{aligned}
& \Phi\left(\frac{z\left(t_{0}\right)}{h}\right)=\Phi\left(\frac{x\left(t_{0} ; t_{0}+h, x_{0}\right)-x_{0}}{h}+f\left(t_{0}, x_{0}\right)\right) \\
& =\Phi\left(\frac{x\left(t_{0} ; t_{0}+h, x_{0}\right)-x\left(t_{0}+h ; t_{0}+h, x_{0}\right)}{h}+f\left(t_{0}, x_{0}\right)\right)=O(1)
\end{aligned}
$$

quando $h \rightarrow 0$. Agora, como em $(a)$, segue do Lema 2.4.4 que

$$
\lim _{h \rightarrow 0} \Phi\left(\frac{z(t)}{h}\right)=r\left(t ; t_{0}, 0\right) \equiv 0 \text {. }
$$

Daí

$$
\lim _{h \rightarrow 0^{+}} \Phi\left(\frac{z(t)}{h}\right)=0
$$

o que prova $(b)$, com

$$
\frac{\partial x}{\partial t_{0}} x\left(t ; t_{0}, x_{0}\right)=-\varphi(t) f\left(t_{0}, x_{0}\right)=-\frac{\partial x}{\partial x_{0}}\left(t ; t_{0}, x_{0}\right) f\left(t_{0}, x_{0}\right)=\psi(t) .
$$




\subsection{Existência Global}

Mesmo no caso em que a função $f(t, x)$ é localmente Lipschitziana em $x$ e contínua para todos os pontos $(t, x)$, exibimos, no Contra-Exemplo 3 da Seção 2.2, uma solução $x(t)$ de (2.29), satisfazendo $x(1)=e_{1}$ e que existe sobre $\left.] 0,1\right]$. Entretanto, $\left.] 0,1\right] \neq I$ e $x(t)$ é limitada para $t \in] 0,1]$. A seguir, iremos impor uma condição sobre $f$ que garantirá a existência global de soluções do problema de Cauchy

$$
\begin{aligned}
& \frac{d x}{d t}=f(t, x), \quad t>t_{0} \\
& x\left(t_{0}\right)=x_{0},
\end{aligned}
$$

onde $f: J \times X \longrightarrow X$ com $J=\left[t_{0}, \infty\left[, X\right.\right.$ é um espaço de Banach e $x_{0} \in X$.

Assumiremos que $f$ satisfaz condições suficientes para garantir a existência local de soluções de (2.61) através de qualquer ponto em $J \times X$. Por exemplo, $f$ pode ser localmente Lipschitziana em $x$, como no Contra-Exemplo 3, ou satisfazer alguma condição de monotonicidade.

Teorema 2.5.1 Suponhamos que:

(i) $f \in C(J \times X, X)$ e para todo $(t, x) \in J \times X$

$$
\|f(t, x)\| \leqslant g(t,\|x\|)
$$

(ii) $g \in C\left(J \times \mathbb{R}_{+}, \mathbb{R}_{+}\right)$e $g(t, r)$ seja não decrescente em $r \geqslant 0$ para cada $t \in J$, e que a solução maximal $r\left(t ; t_{0}, r_{0}\right)$ do problema de valor inicial escalar

$$
\begin{aligned}
& r^{\prime}=g(t, r) \\
& r\left(t_{0}\right)=r_{0},
\end{aligned}
$$

exista em todo $J$, com $r_{0} \in \mathbb{R}_{+}$.

Então, o maior intervalo de existência de qualquer solução $x\left(t ; t_{0}, x_{0}\right)$ de (2.61) com $\left\|x_{0}\right\| \leqslant r_{0}$ é $J$. E ainda, se $r\left(t ; t_{0}, r_{0}\right)$ for limitada sobre $J$, então o limite

$$
\lim _{t \rightarrow \infty} x\left(t ; t_{0}, x_{0}\right)
$$

existe e é um elemento em $X$.

Demonstração: Seja $x(t)=x\left(t ; t_{0}, x_{0}\right)$ uma solução de $(2.61)$ com $\left\|x_{0}\right\| \leqslant r_{0}$, que existe sobre $\left[t_{0}, \beta\left[\right.\right.$, para $t_{0}<\beta<\infty$, e tal que o valor de $\beta$ não pode ser estendido 
(como no Contra-Exemplo 3). Definimos $m(t)=\|x(t)\|$ para $t_{0} \leqslant t<\beta$. Então, por hipótese,

$$
D_{+} m(t) \leqslant\left\|x^{\prime}(t)\right\|=\|f(t, x(t))\| \leqslant g(t,\|x(t)\|)=g(t, m(t)), \quad t_{0} \leqslant t<\beta,
$$

e $m\left(t_{0}\right)=\left\|x\left(t_{0}\right)\right\|=\left\|x_{0}\right\| \leqslant r_{0}$. Assim, pelo Lema 2.4.4, obtemos

$$
\|x(t)\| \leqslant r(t), \quad t_{0} \leqslant t<\beta,
$$

onde $r(t)=r\left(t ; t_{0}, r_{0}\right)$ é a solução maximal de (2.62). Devemos estabelecer que $\lim _{t \rightarrow \beta^{-}} x(t)$ existe e é um elemento em $X$. Uma vez que $g(t, r)$ é não decrescente em $r \geqslant 0$, então para quaisquer $t_{1}$ e $t_{2}$ tais que $t_{0} \leqslant t_{1}<t_{2}<\beta$, temos

$$
\begin{aligned}
\left\|x\left(t_{1}\right)-x\left(t_{2}\right)\right\| & =\left\|\int_{t_{1}}^{t_{2}} f(s, x(s)) d s\right\| \leqslant \int_{t_{1}}^{t_{2}} g(s,\|x(s)\|) d s \\
& \leqslant \int_{t_{1}}^{t_{2}} g(s, r(s)) d s=\int_{t_{1}}^{t_{2}} r^{\prime}(s) d s=r\left(t_{2}\right)-r\left(t_{1}\right) .
\end{aligned}
$$

Uma vez que $\lim _{t \rightarrow \beta^{-}} r(t)$ existe e é finito, tomando os limites quando $t_{1}, t_{2} \rightarrow \beta^{-}$, temos $\left\|x\left(t_{1}\right)-x\left(t_{2}\right)\right\| \rightarrow 0$. Assim, usando o Critério de Cauchy para funções (ver [28], pág. 157, Teorema 8) e (2.64), concluímos que $\lim _{t \rightarrow \beta^{-}} x(t)$ existe.

Definimos, então, $x(\beta)=\lim _{t \rightarrow \beta^{-}} x(t)$, e consideramos agora o problema de valor inicial

$$
\begin{aligned}
& x^{\prime}=f(t, x) \\
& x(\beta)=x_{\beta},
\end{aligned}
$$

onde $x_{\beta}$ é a condição inicial em $t=\beta$. Como estamos assumindo existência local de soluções através de qualquer ponto em $J \times X$, segue que $x(t)$ existe em uma vizinhança de $\beta, \operatorname{assim} x(t)$ pode ser estendida além de $\beta$, o que contradiz nossas suposições. Daí, qualquer solução de (2.61) existe sobre $\left[t_{0}, \infty[\right.$, e então (2.63) e (2.64) valem com $\beta=\infty$. Uma vez que $g(t, r) \geqslant 0$, então $r(t)$ é não decrescente em $J$, e supondo ainda que $r(t)$ seja limitada, segue que $\lim _{t \rightarrow \infty} r(t)$ existe e é finito. Disto e das desigualdades (2.63) e (2.64), $\operatorname{com} \beta=\infty$, segue que

$$
\lim _{t \rightarrow \infty} x(t)
$$

existe e é um elemento em X. 


\section{Observações:}

1. Substituindo $t$ por $-t$ no Teorema 2.5.1, este pode ser estabelecido para o problema de valor inicial

$$
\begin{aligned}
& \frac{d x}{d t}=f(t, x), \quad t \leqslant t_{0} \\
& x\left(t_{0}\right)=x_{0},
\end{aligned}
$$

onde $f: J_{1} \times X \longrightarrow X$ e $\left.\left.J_{1}=\right]-\infty, t_{0}\right]$. Então, substituindo nas hipóteses $(i)$ e (ii) do Teorema 2.5.1, $J$ por $J_{1}$, a mesma conclusão do Teorema 2.5.1 vale para as soluções de (2.65) com $\lim _{t \rightarrow-\infty} x\left(t ; t_{0}, x_{0}\right)$. Os intervalos $J$ e $J_{1}$ acima podem ser substituídos por quaisquer intervalos $\left[t_{0}, t_{0}+\alpha[\mathrm{e}] t_{0}-\alpha, t_{0}\right]$, respectivamente.

2. As hipóteses da Observação 1 não são satisfeitas para o Contra-Exemplo 3 da Seção 2.2. De fato, temos $f: J_{1} \times c_{0} \longrightarrow c_{0}$, onde $\left.\left.J_{1}=\right]-\infty, 1\right]$ e $f(t, x)$ é dada por (2.27). Logo, para $0 \leqslant \frac{1}{n+1}<t \leqslant \frac{1}{n} \leqslant 1$ e $x \in c_{0},\|f(t, x)\|=\left\|\varphi_{n}(t) f_{n}(x)\right\|$. Mas vimos no Contra-Exemplo 3 que $\left\|f_{n}(x)-f_{n}(y)\right\| \leqslant 4\|x-y\|$ para todo $x, y \in c_{0}$, $\log 0$

$$
\|f(t, x)\| \leqslant 4\|x\|\left|\varphi_{n}(t)\right|=4\|x\| \varphi_{n}(t),
$$

uma vez que $\varphi_{n} \geqslant 0$. Tomando $g(t, r)=4 r \varphi_{n}(t)$ para $\frac{1}{n+1}<t \leqslant \frac{1}{n}$ e $g(t, r)=0$ para $t \leqslant 0$, obtemos $g \in C\left(J_{1} \times \mathbb{R}_{+}, \mathbb{R}_{+}\right)$, e $g$ é não decrescente em $r \geqslant 0$ para cada $t \in J_{1}$. Assim, temos o seguinte problema de valor inicial escalar

$$
r^{\prime}(t)= \begin{cases}0, & t \leqslant 0 \\ 4 r(t) \varphi_{n}(t), & \frac{1}{n+1}<t \leqslant \frac{1}{n} .\end{cases}
$$

Uma vez que $g \in C\left(J_{1} \times \mathbb{R}_{+}, \mathbb{R}_{+}\right)$e $g(t, r)$ é localmente Lipschitziana em $r$, então o problema (2.66) possui, localmente, uma única solução. No entanto, mostraremos que o intervalo maximal de existência da solução do problema (2.66), satisfazendo a condição inicial $r(1)=1$, não é todo $\left.\left.J_{1}=\right]-\infty, 1\right]$.

Para $\frac{1}{n+1}<t \leqslant \frac{1}{n}$, sabemos que $\int_{\frac{1}{n+1}}^{\frac{1}{n}} \varphi_{n}(t) d t=1$. Logo as soluções de (2.66), com $n=1,2,3, \ldots$ e $\frac{1}{n+1}<t \leqslant \frac{1}{n}$, são dadas por

$$
\begin{array}{ll}
r(t)=r(1) e^{-4 \int_{t}^{1} \varphi_{n}(s) d s}=\left\|e_{1}\right\| e^{-4 \int_{t}^{1} \varphi_{n}(s) d s}=e^{-4 \int_{t}^{1} \varphi_{n}(s) d s}, & \frac{1}{2}<t \leqslant 1 \\
r(t)=r\left(\frac{1}{2}\right) e^{-4 \int_{t}^{\frac{1}{2}} \varphi_{n}(s) d s}=e^{-4 \int_{\frac{1}{2}}^{1} \varphi_{n}(s) d s} e^{-4 \int_{t}^{\frac{1}{2}} \varphi_{n}(s) d s}=e^{-4} e^{-4 \int_{t}^{\frac{1}{2}} \varphi_{n}(s) d s}, & \frac{1}{3}<t \leqslant \frac{1}{2} \\
r(t)=r\left(\frac{1}{3}\right) e^{-4 \int_{t}^{\frac{1}{3}} \varphi_{n}(s) d s}=e^{-4} e^{-4 \int_{\frac{1}{3}}^{\frac{1}{2}} \varphi_{n}(s) d s} e^{-4 \int_{t}^{\frac{1}{3}} \varphi_{n}(s) d s}=e^{-8} e^{-4 \int_{t}^{\frac{1}{3}} \varphi_{n}(s) d s}, & \frac{1}{4}<t \leqslant \frac{1}{3} .
\end{array}
$$


E assim sucessivamente, para $\frac{1}{n+1}<t \leqslant \frac{1}{n}$, segue que

$$
r(t)=r\left(\frac{1}{n}\right) e^{-4 \int_{t}^{\frac{1}{n}} \varphi_{n}(s) d s}=e^{-4(n-1)} e^{-4 \int_{t}^{\frac{1}{n}} \varphi_{n}(s) d s}
$$

é uma solução de (2.66). E ainda, $r\left(\frac{1}{n}\right)=e^{-4(n-1)} \rightarrow 0$, quando $n \rightarrow \infty$. Uma vez que $r(t)$ é contínua, então $r\left(\frac{1}{n}\right) \rightarrow r(0)$, quando $n \rightarrow \infty$, logo $r(0)=0$. Assim, se fosse possível estender a solução $r(t)$ dada por (2.67) além do zero, esta iria satisfazer a condição $r(0)=0$. Mas, por outro lado, a função $r(t) \equiv 0$ também é uma solução de (2.66) e satisfaz a condição $r(0)=0$, e isto é um absurdo, pela unicidade de soluções. Portanto não é possível estender $r(t)$ além de ]0,1], sendo assim este é o intervalo maximal. Daí, a solução do problema (2.66), satisfazendo a condição inicial $r(1)=1$, não existe sobre todo $\left.\left.J_{1}=\right]-\infty, 1\right]$, logo não podemos aplicar o Teorema 2.5.1.

Corolário 2.5.2 Suponhamos que $f \in C(J \times X, X)$ seja globalmente Lipschitziana na variávelx. Então, existe uma única solução do problema de Cauchy (2.61) definida sobre todo $J$, que depende continuamente do dado inicial $\left(t_{0}, x_{0}\right)$.

Demonstração: Suponhamos que $f(t, x)$ seja globalmente Lipschitziana na variável $x$, com constante $L \geqslant 0$. Então, pelo Teorema 2.2.1, obtemos existência local e unicidade de soluções para o problema (2.61). E ainda, as hipóteses do Teorema 2.5.1 são satisfeitas. De fato, para todo $(t, x) \in J \times X$, temos $\|f(t, x)\| \leqslant L\|x\|$, logo $f$ satisfaz a condição $(i)$. Tomando $g(t, r)=L r$, segue que $g \in C\left(J \times \mathbb{R}_{+}, \mathbb{R}_{+}\right)$e não decrescente em $r \geqslant 0$ para todo $t \in J$, e ainda é globalmente Lipschitziana em $r$. Logo, o problema de valor inicial escalar (2.62) possui uma única solução em todo $J$, e ainda, esta é dada por $r\left(t ; t_{0}, r_{0}\right)=r_{0} e^{L\left(t-t_{0}\right)}$. Portanto, pelo Teorema 2.5.1, obtemos existência global de soluções para o problema (2.61), para $\left\|x_{0}\right\| \leqslant r_{0}$.

Mostraremos que as soluções de (2.61) são únicas sobre $J$. Tomando o funcional admissível $\Phi(x)=\|x\|$ em $X, \operatorname{com} M[x, h]=\|h\|$, temos

$M[x-y, f(t, x)-f(t, y)]=\|f(t, x)-f(t, y)\| \leqslant L\|x-y\|=L \Phi(x-y)=g(t, \Phi(x-y))$.

E ainda, $r\left(t ; t_{0}, 0\right) \equiv 0$. Logo, pelo Teorema 2.4.6, o problema (2.61) possui uma única solução sobre $J$. Agora, uma vez que a solução maximal $r\left(t ; t_{0}, r_{0}\right)$ de $(2.62)$ também depende continuamente do dado inicial $\left(t_{0}, r_{0}\right)$, então, pelo Teorema 2.4.7, a solução de (2.61) depende continuamente do dado inicial $\left(t_{0}, x_{0}\right)$. 


\subsection{Condições de Compacidade}

Vimos nos Contra-Exemplos 1 e 2 da Seção 2.2 que apenas a continuidade da função $f(t, x)$ não é suficiente para garantirmos a existência de uma solução local para o problema (2.1), usando o Teorema de Peano, pois este não é válido em espaços de Banach de dimensão infinita, como vimos no Teorema 2.2.8. Entretanto, conseguimos solucionar o problema de existência local acrescentando a hipótese que $f(t, x)$ é localmente Lipschitziana na variável $x$, como vimos no Teorema 2.2.1. Mas, como comentamos anteriormente, esta não é a única maneira de obtermos existência local para o problema (2.1). Uma vez que a perda de compacidade é a principal razão para não existência, é natural tentar "medir" a não compacidade de subconjuntos $B$ de um espaço de Banach $X$, a grosso modo, saber quais são as seqüências que admitem subseqüências convergindo para pontos que não estão em $B$. Depois disto, olhar para as condições sobre $f(t, x)$, suficientes para a existência, em termos de tal medida. A seguir, trabalharemos com esses conceitos e, para simplificar, iremos considerar $t_{0}=0$ em (2.1). Assim de (2.1) obtemos o seguinte problema de valor inicial

$$
\begin{aligned}
& x^{\prime}=f(t, x), \quad 0<t \leqslant a \\
& x(0)=x_{0} .
\end{aligned}
$$

Para este trabalho utilizaremos [11].

\subsubsection{Medida de Não Compacidade e Existência Local}

Inicialmente, relembremos que um subconjunto $S$ de um espaço de Banach $X$ é relativamente compacto se $\bar{S}$ é compacto. Mas como $X$ é completo, isto é equivalente a dizermos que para todo $\epsilon>0$, existe um número finito de bolas fechadas de raio $\epsilon$ tais que sua união cobre $S$ ( $S$ é totalmente limitado). Se $S$ é somente limitado, existe um limite inferior positivo para tais números $\epsilon$. Estes fatos sugerem a seguinte definição:

Definição 2.6.1 Sejam X um espaço de Banach e $\mathcal{L}$ a família de todos os subconjuntos limitados de $X$. Para $B \in \mathcal{L}$,

(a) a medida de Hausdorff de não compacidade $\gamma: \mathcal{L} \longrightarrow \mathbb{R}$ é definida por $\gamma(B)=\inf \{\epsilon>0: B$ admite uma cobertura finita por bolas fechadas de raio $\leqslant \epsilon\}$; 
(b) a medida de Kuratowski de não compacidade $\alpha: \mathcal{L} \longrightarrow \mathbb{R}$ é definida por $\alpha(B)=\inf \{d>0: B$ admite uma cobertura finita por conjuntos de diâmetro $\leqslant d\}$.

As medidas de não compacidade $\gamma$ e $\alpha$ são, em algum sentido, equivalentes, uma vez que vale a seguinte desigualdade

$$
\gamma(B) \leqslant \alpha(B) \leqslant 2 \gamma(B), \quad \operatorname{com} B \in \mathcal{L} .
$$

Vejamos também algumas propriedades de tais medidas.

Lema 2.6.2 Seja $\alpha: \mathcal{L} \longrightarrow \mathbb{R}$ a medida de Kuratowski. Então:

(i) $\alpha(B)=0 \Longleftrightarrow \bar{B}$ é compacto;

(ii) $\alpha\left(\bar{B}_{1}(0)\right) \leqslant 2$;

(iii) $\alpha$ é uma semi-norma, isto é,

$$
\alpha(\lambda B)=|\lambda| \alpha(B) \quad \text { e } \quad \alpha(B+C) \leqslant \alpha(B)+\alpha(C) .
$$

$E$ ainda, $|\alpha(B)-\alpha(C)| \leqslant \alpha(B-C)$;

(iv) $B \subset C \Longrightarrow \alpha(B) \leqslant \alpha(C)$;

(v) $\alpha(B \cup C)=\max \{\alpha(B), \alpha(C)\} \quad e \quad \alpha(B \cap C) \leqslant \min \{\alpha(B), \alpha(C)\}$;

(vi) $\alpha$ é contínua na métrica de Hausdorff

$$
d_{H}(B, C)=\max \left\{\sup _{B} \rho(x, C), \sup _{C} \rho(x, B)\right\}
$$

onde $\rho(x, B)=\inf \{\|x-y\|: y \in B\}$ e $\rho(x, C)=\inf \{\|x-y\|: y \in C\}$;

(vii) $\alpha(B)=\alpha(\bar{B})$;

(viii) $\alpha(\operatorname{co}(B))=\alpha(B)$.

A demonstração das propriedades acima pode ser encontrada em [11], pág. 19, Lema 2.2.

A medida $\gamma$ tem as mesmas propriedades, exceto a propriedade (ii), uma vez que $\gamma\left(\bar{B}_{1}(0)\right) \leqslant 1$.

Mais detalhes sobre as medidas de não compacidade $\gamma$ e $\alpha$ podem ser encontrados em [1].

Suponhamos, agora, que $f: I \times X \longrightarrow X$, onde $I=[0, a]$, seja contínua e leve conjuntos limitados em conjuntos relativamente compactos. Chamamos tal $f$ de compacta. Seja

$$
f(t, B)=\{f(t, x): x \in B\}, \quad \text { para } B \in \mathcal{L} .
$$


Como $f(t, B)$ é um conjunto relativamente compacto, então $\overline{f(t, B)}$ é compacto. Logo, pelo Lema 2.6.2, $(i)$,

$$
\alpha(f(t, B))=0 \leqslant \alpha(B), \quad \text { para } B \in \mathcal{L} .
$$

Agora, suponhamos que $f$ admita uma decomposição $f=f_{1}+f_{2}$ tal que $f_{1}$ seja compacta e $f_{2}$ seja Lipschitziana na segunda variável, com constante $k \geqslant 0$. Então, por $(i)$ e (iii) do Lema 2.6.2, temos

$$
\alpha(f(t, B))=\alpha\left(f_{1}(t, B)+f_{2}(t, B)\right) \leqslant \alpha\left(f_{1}(t, B)\right)+\alpha\left(f_{2}(t, B)\right)=\alpha\left(f_{2}(t, B)\right) .
$$

Como $\left\|f_{2}(t, x)-f_{2}(t, y)\right\| \leqslant k\|x-y\|$, com $k \geqslant 0$, então $f_{2}(t, B) \subset k B$. Assim

$$
\alpha(f(t, B)) \leqslant k \alpha(B), \quad \text { para } B \in \mathcal{L} .
$$

Devemos mostrar que estimativas do tipo (2.69) e (2.70) implicam existência local para o problema de valor inicial (2.68). Inicialmente, temos a seguinte proposição:

Proposição 2.6.3 Seja $x:[0, a] \longrightarrow X$ diferenciável. Então

$$
\left.\left.\left\{\frac{x(t)-x(t-h)}{h}: \quad t \in\right] 0, a\right], \quad 0<h \leqslant t\right\} \subset \overline{c o}\left(\left\{x^{\prime}(t): \quad t \in[0, a]\right\}\right) .
$$

Demonstração: Seja $K=\overline{c o}\left(\left\{x^{\prime}(t): t \in[0, a]\right\}\right)$. Pela Definição 2.2.4, $K$ é fechado e convexo e, portanto, pelo corolário do Teorema de Hahn-Banach de Separação (ver [24], pág. 193), $K$ é a intersecção de todos os semi-espaços

$$
\left\{x \in X: \Re\left[x^{*} x\right] \leqslant \lambda\right\}
$$

que o contém. Daí, basta mostrarmos que

$$
\Re\left[x^{*}(x(t)-x(t-h))\right] \leqslant \lambda h, \quad \text { sempre que } K \subset\left\{x: \Re\left[x^{*} x\right] \leqslant \lambda\right\} .
$$

Seja $t_{0} \in\left[0, a\left[\right.\right.$ e $\phi(t)=\Re\left[x^{*}\left(x(t)-x\left(t_{0}\right)\right)\right]$ para $t \in\left[t_{0}, a\right]$.

Como $x^{\prime}(t) \in K$, então $\phi^{\prime}(t)=\Re\left[x^{*} x^{\prime}(t)\right] \leqslant \lambda$. E ainda $\phi\left(t_{0}\right)=0$, logo $\phi(t) \leqslant$ $\lambda\left(t-t_{0}\right)$.

Teorema 2.6.4 Sejam $X$ um espaço de Banach, $\bar{B}_{r}\left(x_{0}\right) \subset X, I=[0, a]$ um intervalo em $\mathbb{R}$ e $f: I \times \bar{B}_{r}\left(x_{0}\right) \longrightarrow X$ uma função uniformemente contínua e limitada, digamos $\|f(t, x)\| \leqslant M$. Suponhamos que exista uma constante $L \geqslant 0$ tal que

$$
\alpha(f(t, B)) \leqslant L \alpha(B), \quad \forall t \in I \quad e \quad B \subset \bar{B}_{r}\left(x_{0}\right),
$$

e seja $\delta<\min \left\{a, \frac{r}{M}\right\}$. Então, o problema (2.68) possui uma solução sobre $[0, \delta]$. 
Demonstração: Nas condições do teorema, podemos aplicar o Teorema 2.2.6 e construir soluções aproximadas $x_{n}, n \geqslant 1$, sobre $[0, \delta]$ tais que

$$
\begin{aligned}
& x_{n}^{\prime}=f\left(t, x_{n}(t)\right)+y_{n}(t) \\
& x_{n}(0)=x_{0},
\end{aligned}
$$

$\operatorname{com}\left\|y_{n}(t)\right\| \leqslant \frac{1}{n}$. Assim

$$
x_{n}(t)=x_{0}+\int_{0}^{t} f\left(s, x_{n}(s)\right) d s+\int_{0}^{t} y_{n}(s) d s .
$$

Sabemos que a seqüência $\left(x_{n}\right)$ é eqüicontínua. Agora, se mostrarmos que o conjunto $\left\{x_{n}(t): n \geqslant 1\right\}$ é relativamente compacto para cada $t \in[0, \delta]$, ou equivalentemente, $\alpha\left(\left\{x_{n}(t): n \geqslant 1\right\}\right)=0$ para cada $t \in[0, \delta]$, pelo Teorema 2.2 .2 seguirá que a seqüência $\left(x_{n}\right)$ admite uma subseqüência uniformemente convergente, cujo limite será uma solução de (2.68) sobre [0, $\delta$, como veremos.

Consideremos

$$
B_{k}(t)=\left\{x_{n}(t): n \geqslant k\right\}, \quad B_{k}^{\prime}(t)=\left\{x_{n}^{\prime}(t): n \geqslant k\right\}, \quad \text { e } \quad \phi_{k}(t)=\alpha\left(B_{k}(t)\right) .
$$

Temos

$$
\phi_{k}(0)=\alpha\left(B_{k}(0)\right)=\alpha\left(\left\{x_{n}(0): n \geqslant k\right\}\right)=\alpha\left(x_{0}\right)=0 .
$$

E $\phi_{k}$ é contínua, uma vez que por (iii) do Lema 2.6.2, temos

$$
\begin{aligned}
& \left|\phi_{k}(t)-\phi_{k}(s)\right|=\left|\alpha\left(B_{k}(t)\right)-\alpha\left(B_{k}(s)\right)\right| \leqslant \alpha\left(B_{k}(t)-B_{k}(s)\right) \\
& \leqslant \alpha\left(\left\{x_{n}(t)-x_{n}(s): n \geqslant k\right\}\right)=\alpha\left(\left\{x_{n}^{\prime}(\xi)(s-t): \quad n \geqslant k \quad \text { e } \quad \xi \in\right] t, s[\}\right) \\
& \leqslant|s-t| \alpha\left(\left\{f\left(\xi, x_{n}(\xi)\right)+y_{n}(\xi): \quad n \geqslant k \quad \text { e } \quad \xi \in\right] t, s[\}\right) \\
& \leqslant\left(M+\frac{1}{n}\right)|t-s| \leqslant(M+1)|t-s| .
\end{aligned}
$$

Afirmamos que

$$
\left.\left.D^{+} \phi_{k}(t) \leqslant \alpha\left(f\left(t, B_{k}(t)\right)\right)+\frac{2}{k}, \quad \text { em }\right] 0, \delta\right],
$$

onde

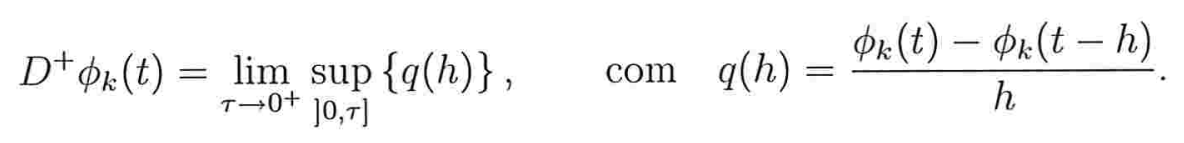


De $($ iii $)$ e $(v)$ do Lema 2.6.2, obtemos

$$
\begin{aligned}
\sup _{j 0, \tau]}\{q(h)\} & =\sup _{j 0, \tau]}\left\{\frac{\phi_{k}(t)-\phi_{k}(t-h)}{h}\right\}=\sup _{j 0, \tau]}\left\{\frac{\alpha\left(B_{k}(t)\right)-\alpha\left(B_{k}(t-h)\right)}{h}\right\} \\
& \leqslant \sup _{j 0, \tau]}\left\{\alpha\left(\frac{B_{k}(t)-B_{k}(t-h)}{h}\right)\right\} \\
& =\alpha\left(\left\{\frac{x_{n}(t)-x_{n}(t-h)}{h}: \quad n \geqslant k, \quad 0<h \leqslant \tau\right\}\right) .
\end{aligned}
$$

Logo, usando a Proposição 2.6.3, (iv), (v) e (viii) do Lema 2.6.2, temos

$D^{+} \phi_{k}(t)=\lim _{\tau \rightarrow 0^{+}} \sup _{j 0, \tau]}\{q(h)\} \leqslant \lim _{\tau \rightarrow 0^{+}} \alpha\left(\overline{c o}\left\{x_{n}^{\prime}(t): n \geqslant k\right\}\right)$

$=\lim _{\tau \rightarrow 0^{+}} \alpha\left(\left\{x_{n}^{\prime}(t): n \geqslant k\right\}\right) \leqslant \lim _{\tau \rightarrow 0^{+}} \sup _{I_{\tau}}\left\{\alpha\left(\left\{x_{n}^{\prime}(s): n \geqslant k\right\}\right)\right\}=\lim _{\tau \rightarrow 0^{+}} \alpha\left(\bigcup_{I_{\tau}} B_{k}^{\prime}(s)\right)$,

$\operatorname{com} I_{\tau}=[t-\tau, t]$.

E ainda, por (iii) do Lema 2.6.2, temos

$$
\begin{aligned}
& \alpha\left(\bigcup_{I_{\tau}} B_{k}^{\prime}(s)\right)=\alpha\left(\bigcup_{I_{\tau}}\left\{x_{n}^{\prime}(s): n \geqslant k\right\}\right) \\
& =\alpha\left(\bigcup_{I_{\tau}}\left\{f\left(t, x_{n}(s)\right)+y_{n}(s): n \geqslant k\right\}\right)=\alpha\left(\bigcup_{I_{\tau}} f\left(s, B_{k}(s)\right)+\bigcup_{I_{\tau}} \bigcup_{n \geqslant k}\left\{y_{n}(s)\right\}\right) \\
& \leqslant \alpha\left(\bigcup_{I_{\tau}} f\left(s, B_{k}(s)\right)\right)+\alpha\left(\bigcup_{I_{\tau}}\left\{y_{n}(s)\right\}\right) \leqslant \alpha\left(\bigcup_{I_{\tau}} f\left(s, B_{k}(s)\right)\right)+\frac{2}{k},
\end{aligned}
$$

pois, uma vez que $\left\|y_{n}(t)\right\| \leqslant \frac{1}{n}$, então $y_{n}(t) \in \bar{B}_{\frac{1}{n}}(0) \subset \bar{B}_{\frac{1}{k}}(0)$. Logo, por (ii) do Lema 2.6.2, temos $\alpha\left(\bar{B}_{\frac{1}{k}}(0)\right) \leqslant \frac{2}{k}$.

Uma vez que $\left(x_{n}\right)$ é equiicontínua e $f$ é uniformemente contínua, então

$$
\bigcup_{I_{\tau}} f\left(s, B_{k}(s)\right) \rightarrow f\left(t, B_{k}(t)\right), \quad \text { quando } \tau \rightarrow 0^{+}
$$

na métrica de Hausdorff. Assim, de (2.74), obtemos (2.73).

E ainda, por (2.71), segue que $\alpha\left(f\left(t, B_{k}(t)\right)\right) \leqslant L \alpha\left(B_{k}(t)\right)=L \phi_{k}(t)$. Logo, por (2.73), obtemos

$$
D^{+} \phi_{k}(t) \leqslant L \phi_{k}(t)+\frac{2}{k}
$$


Resolvendo a equação acima, obtemos $\phi_{k}(t) \leqslant 2(k L)^{-1} e^{L t}-2(k L)^{-1}$ e, portanto,

$$
\alpha\left(\left\{x_{n}(t): n \geqslant 1\right\}\right)=\alpha\left(B_{k}(t)\right) \leqslant 2(k L)^{-1} e^{L t}-2(k L)^{-1} \rightarrow 0,
$$

quando $k \rightarrow \infty$.

Portanto, $\left\{x_{n}(t): n \geqslant 1\right\}$ é relativamente compacto. Então, pelo Teorema 2.2.2, $\left\{x_{n}: n \geqslant 1\right\}$ é relativamente compacto. Daí, existe uma subseqüência de $\left(x_{n}\right)$, que também denotaremos por $\left(x_{n}\right)$, convergindo uniformemente em $[0, \delta]$ para uma função $x(t)$. Provaremos que $x(t)$ é uma solução de (2.68).

Uma vez que $f$ é uniformemente contínua em $I \times \bar{B}_{r}\left(x_{0}\right)$ e $x_{n}(t) \rightarrow x(t)$, quando $n \rightarrow \infty$ e $t \in[0, \delta]$, então $f\left(s, x_{n}(s)\right) \rightarrow f(s, x(s))$, quando $n \rightarrow \infty$ e ainda temos $\left\|f\left(s, x_{n}(s)\right)\right\| \leqslant M$. Fazendo $n \rightarrow \infty$ em ambos os membros de (2.72) e usando o Teorema da Convergência Dominada, segue que para todo $t \in[0, \delta]$,

$$
x(t)=x_{0}+\int_{0}^{t} f(s, x(s)) d s .
$$

Portanto, $x(t)$ é uma solução de (2.68) sobre $[0, \delta]$.

Observação: Notemos que uma função contínua $f: I \times \bar{B}_{r}\left(x_{0}\right) \longrightarrow X$ é limitada, se $r$ é suficientemente pequeno. Porém, $f$ não precisa ser uniformemente contínua se a dimensão de $X$ for infinita. A continuidade uniforme de $f$ implica

$$
\alpha(f(I \times B))=\alpha\left(\bigcup_{I} f(t, B)\right)=\max _{I}\{\alpha(f(t, B))\}, \quad \text { para } B \subset \bar{B}_{r}\left(x_{0}\right) .
$$

Daí, o Teorema 2.6.4 é um caso particular do próximo teorema, onde dispensamos a continuidade uniforme de $f$.

Teorema 2.6.5 Sejam X um espaço de Banach, $\bar{B}_{r}\left(x_{0}\right) \subset X, I=[0, a]$ um intervalo em $\mathbb{R}, f: I \times \bar{B}_{r}\left(x_{0}\right) \longrightarrow X$ uma função contínua e limitada, digamos $\|f(t, x)\| \leqslant M$. Suponhamos que $f$ satisfaça

$$
\alpha(f(I \times B)) \leqslant \omega(\alpha(B)), \quad \text { para } B \subset \bar{B}_{r}\left(x_{0}\right),
$$

com $\omega: \mathbb{R}_{+} \longrightarrow \mathbb{R}_{+}$contínua e tal que o problema de valor inicial $\rho^{\prime}=\omega(\rho)$ e $\rho(0)=0$ possua somente a solução trivial $\rho(t) \equiv 0$ sobre $I$. Então, (2.68) possui uma solução sobre $[0, \delta]$, onde $\delta<\min \left\{a, \frac{r}{M}\right\}$. 
Demonstração: Repetimos a prova do Teorema 2.6.4 até (2.74). Agora, usando (2.75), obtemos

$$
\alpha\left(\bigcup_{I_{\tau}} f\left(s, B_{k}(s)\right)\right)=\alpha\left(f\left(I_{\tau} \times \bigcup_{I_{\tau}} B_{k}(s)\right)\right) \leqslant \omega\left(\alpha\left(\bigcup_{I_{\tau}} B_{k}(s)\right)\right) .
$$

Daí, $D^{+} \phi_{k}(t) \leqslant \omega\left(\alpha\left(\bigcup_{I_{\tau}} B_{k}(s)\right)\right)+\frac{2}{k}$. Uma vez que $\left(x_{n}\right)$ é eqüicontínua, então

$$
\bigcup_{I_{\tau}} B_{k}(s) \rightarrow B_{k}(t), \quad \text { quando } \tau \rightarrow 0^{+} .
$$

Logo, $D^{+} \phi_{k}(t) \leqslant \omega\left(\phi_{k}(t)\right)+\frac{2}{k}$, em $\left.] 0, \delta\right]$ e $\phi_{k}(0)=0$. E ainda, pela hipótese sobre $\omega$, existem funções $\rho_{n}$ tais que

$$
\rho_{n}^{\prime}=\omega\left(\rho_{n}\right)+\frac{1}{n} \quad \text { e } \quad \rho_{n}(0)=\frac{1}{n}
$$

e $\rho_{n}(t) \rightarrow 0$ quando $n \rightarrow \infty$. Assim, $\phi_{k}(0) \leqslant \rho_{n}(0)$ e $D^{+} \phi_{k}(t) \leqslant \omega\left(\phi_{k}(t)\right)+\frac{1}{n}$, para $k>2 n$. Logo, pelo Lema 2.4.4, $\phi_{k}(t)<\rho_{n}(t)$ para $k>2 n$. Isto implica que $\phi_{k}(t) \rightarrow 0$ quando $k \rightarrow \infty$.

Vimos que os Teoremas 2.6.4 e 2.6.5 garantem que, sob certas condições, existe uma solução para o problema (2.68), porém não asseguram unicidade. Assim, é interessante examinarmos as propriedades do conjunto de todas as soluções do problema (2.68).

Teorema 2.6.6 Suponhamos que as condições do Teorema 2.6.4 ou do Teorema 2.6.5 sejam satisfeitas. Então, o conjunto $D \subset C([0, \delta], X)$ de todas as soluções de (2.68) sobre $[0, \delta]$ é compacto e conexo.

Demonstração: Examinando a parte de existência na prova do Teorema 2.6.4, se considerarmos uma seqüência de soluções de (2.68), em vez das soluções aproximadas $x_{n}$, obtemos uma subseqüência uniformemente convergente e seu limite é também uma solução. Portanto $D$ é compacto.

Suponhamos, por contradição, que $D$ não seja conexo. Então $D=D_{1} \cup D_{2}$, com $D_{1} \cap D_{2}=\emptyset$ e $D_{1}, D_{2}$ compactos ( $D$ é compacto). Daí $\beta=\rho\left(D_{1}, D_{2}\right)>0$. Consideremos o funcional $\phi: C([0, \delta], X) \longrightarrow \mathbb{R}$ definido por $\phi(x)=\rho\left(x, D_{1}\right)$ $\rho\left(x, D_{2}\right)$. 
O funcional $\phi$ é contínuo, $\phi(x) \leqslant-\beta$ sobre $D_{1}$ e $\phi(x) \geqslant \beta$ sobre $D_{2}$. Para chegarmos em um absurdo, construiremos um $x \in D$ tal que $\phi(x)=0$. Com esta finalidade, seja $\epsilon>0$ tal que $(M+2 \epsilon) \delta \leqslant r$. Pelo Lema 2.2.3, encontramos $g_{\epsilon}$ : $I \times \bar{B}_{r}\left(x_{0}\right) \longrightarrow X$ localmente Lipschitziana e tal que $\left\|g_{\epsilon}(t, x)-f(t, x)\right\| \leqslant \epsilon$ sobre $I \times \bar{B}_{r}\left(x_{0}\right)$. Fixemos $x_{i} \in D_{i}$ e consideremos

$$
f^{i}(t, x)=g_{\epsilon}(t, x)+f\left(t, x_{i}(t)\right)-g_{\epsilon}\left(t, x_{i}(t)\right), \quad \text { para } i=1,2 .
$$

Para $\lambda \in[0,1]$, seja

$$
f_{\lambda}(t, x)=f^{1}(t, x)+\lambda\left(f^{2}(t, x)-f^{1}(t, x)\right) .
$$

A função $f_{\lambda}$ é localmente Lipschitziana na segunda variável. De fato,

$$
\begin{aligned}
& \left\|f_{\lambda}(t, x)-f_{\lambda}(t, y)\right\| \leqslant\left\|f^{1}(t, x)-f^{1}(t, y)\right\| \\
& +|\lambda|\left\|f^{2}(t, x)-f^{2}(t, y)\right\|+|\lambda|\left\|f^{1}(t, y)-f^{1}(t, x)\right\|,
\end{aligned}
$$

e o resultado segue da Lipschitz continuidade de $f^{1}$ e $f^{2}$ na segunda variável. E ainda,

$$
\begin{aligned}
& \left\|f_{\lambda}(t, x)-f(t, x)\right\|=\left\|f^{1}(t, x)+\lambda\left(f^{2}(t, x)-f^{1}(t, x)\right)-f(t, x)\right\| \\
& \leqslant\left\|g_{\epsilon}(t, x)-f(t, x)\right\|+|\lambda|\left\|f\left(t, x_{2}(t)\right)-g_{\epsilon}\left(t, x_{2}(t)\right)\right\|+\| f\left(t, x_{1}(t)\right)-g_{\epsilon}\left(t, x_{1}(t)\right) \\
& +\lambda\left(g_{\epsilon}\left(t, x_{1}(t)\right)-f\left(t, x_{1}(t)\right)\right) \| \\
& \leqslant \epsilon+\epsilon+(1-\lambda)\left\|f\left(t, x_{1}(t)\right)\right\|+(\lambda-1)\left\|g_{\epsilon}\left(t, x_{1}(t)\right)\right\| \leqslant 2 \epsilon+(\lambda-1) \epsilon=2 \epsilon .
\end{aligned}
$$

Portanto, o problema de valor inicial $x^{\prime}=f_{\lambda}(t, x)$ e $x(0)=x_{0}$ tem uma única solução $x^{\lambda}$ sobre $[0, \delta]$ e a aplicação $\lambda \longmapsto x^{\lambda}$ é contínua. Daí, $\psi(\lambda)=\phi\left(x^{\lambda}\right)$ é contínua em $[0,1]$. Como

$$
f_{0}\left(t, x_{1}(t)\right)=f^{1}\left(t, x_{1}(t)\right)=x_{1}^{\prime}(t)
$$

e $x^{0}$ é a solução da equação acima, então $x^{0}=x_{1}$, pela unicidade. De maneira análoga, $x^{1}=x_{2}$. Logo, $\psi(0)=\phi\left(x^{0}\right)=\phi\left(x_{1}\right) \leqslant-\beta$ e $\psi(1)=\phi\left(x^{1}\right)=\phi\left(x_{2}\right) \geqslant \beta$, com $x_{1} \in D_{1}$ e $x_{2} \in D_{2}$. Portanto, encontramos um $\left.\lambda(\epsilon) \in\right] 0,1[$ tal que $\psi(\lambda(\epsilon))=0$.

Seja $x_{n}=x^{\lambda\left(\epsilon_{n}\right)}$ para alguma seqüência $\epsilon_{n} \rightarrow 0^{+}$. Como

$$
\begin{aligned}
& x_{n}^{\prime}=f\left(t, x_{n}(t)\right)+y_{n}(t) \\
& x_{n}(0)=x_{0},
\end{aligned}
$$


$\mathrm{e}\left\|y_{n}(t)\right\|=\left\|f_{\lambda\left(\epsilon_{n}\right)}\left(t, x_{n}(t)\right)-f\left(t, x_{n}(t)\right)\right\| \leqslant 2 \epsilon_{n}$. Então, com base nos Teoremas 2.6.4 e 2.6.5, segue que $\left(x_{n}\right)$ possui uma subseqüência convergindo uniformemente para a solução $x$ de (2.68), isto é, $x \in D$ e

$$
\phi(x)=\lim _{n \rightarrow \infty} \phi\left(x_{n}\right)=0,
$$

o que é um absurdo. Portanto $D$ é conexo.

\subsection{Equações Diferenciais Ordinárias em Espaços de Banach Reflexivos}

Na Seção 2.2, vimos que apenas a continuidade de $f(t, x)$ não é suficiente para garantir a existência de soluções clássicas para o problema de Cauchy (2.1), uma vez que temos perda de compacidade da bola fechada unitária em espaços de dimensão infinita. Contudo, existem espaços de Banach com propriedades especiais, em que é possível construirmos resultados de existência. No entanto, nem sempre obtemos soluções clássicas nestas construções, como é o caso dos espaços de Banach reflexivos. Em [36], Szép mostrou que dado um espaço de Banach reflexivo $X$, e considerando o problema (2.1) neste espaço, com $f: P \longrightarrow X$, onde $P \subset \mathbb{R} \times X$ é um conjunto não vazio, sob certas condições, sempre é possível obter soluções fracas para o problema (2.1). Veremos este resultado nesta seção. Para tanto, utilizaremos vários resultados e conceitos de Análise Funcional, que detalhadamente podem ser encontrados em [14], [26] e [39]. Incialmente, definiremos quais são as condições para que um espaço normado $B$ seja reflexivo.

Seja $B$ um espaço normado e $B^{* *}=\left(B^{*}\right)^{*}$ o seu espaço bidual. Definimos um funcional $g_{x}$ sobre o espaço dual de $B^{*}$, fixando um $x \in B$ e tomando

$$
g_{x}(f)=f(x), \quad \text { com } f \in B^{*} \text { variável. }
$$

Para todo $x$ fixado no espaço normado $B$, o funcional $g_{x}$ definido por (2.76) é um funcional linear limitado sobre $B^{*}$ tal que $g_{x} \in B^{* *}$ e $\left\|g_{x}\right\|=\|x\|$ (ver [26], pág. 240, Lema 4.6-1).

Para todo $x \in B$, existe um único funcional linear limitado $g_{x} \in B^{* *}$ dado por 
(2.76). Assim, definimos uma aplicação

$$
\begin{aligned}
C: B & \longrightarrow B^{* *} \\
x & \longmapsto g_{x} .
\end{aligned}
$$

Chamamos $C$ de aplicação canônica de $B$ em $B^{* *}$. E ainda, $C$ é linear, injetora e preserva a norma. Podemos expressar estes fatos dizendo que a aplicação $C$ é um isomorfismo do espaço normado $B$ sobrejetivamente no espaço normado $\operatorname{Im}(C)$, onde $\operatorname{Im}(C) \subset B^{* *}$ é a imagem de $C$ (ver [26], pág. 240, Lema 4.6-2). Assim, temos a seguinte definição:

Definição 2.7.1 Dizemos que um espaço normado $B$ é reflexivo se $\operatorname{Im}(C)=B^{* *}$, onde $C: B \longrightarrow B^{* *}$ é a aplicação canônica dada por (2.76) e (2.77).

Consideremos agora $X$ um espaço de Banach reflexivo, com a topologogia fraca (ver [39], pág. 112, Definição 3), e $X^{*}$ o seu dual, com a topologia fraca* (ver [39], pág. 111, Definição 2). Uma vez que $X$ é um espaço de Banach reflexivo, a topologia fraca coincide com a topologia fraca*. Suponhamos que a função $f(t, x)$ seja fracamentefracamente contínua sobre $P$, isto é, fracamente contínua nas variáveis $t$ e $x$. Assim, para todo $\left(t^{\prime}, x^{\prime}\right) \in P$ e vizinhança fraca $U$ arbitrária do ponto $f\left(t^{\prime}, x^{\prime}\right) \in X$, existem um $\epsilon>0$ e uma vizinhança fraca $V$ de $x^{\prime}$ tais que para todo $x \in V,\left|t-t^{\prime}\right|<\epsilon$, $(t, x) \in P$ temos $f(t, x) \in U$. Ou ainda, na forma seqüencial, podemos supor que a função $f(t, x)$ seja fracamente-fracamente seqüencialmente contínua, isto é, para cada seqüência fracamente convergente $\left(t_{n}, x_{n}\right)$ em $P$, a seqüência $\left(f\left(t_{n}, x_{n}\right)\right)$ é fracamente convergente em $X$.

Veremos alguns resultados em espaços de Banach reflexivos, bem como algumas definições e resultados de análise em espaços de Banach quaisquer, que usaremos para obter um teorema de existência de soluções fracas de (2.1).

\subsubsection{Alguns Resultados e Definições}

A seguir, veremos algumas propriedades. A primeira delas é a completitude fraca dos espaços reflexivos. Mas antes, precisamos das seguintes definições:

Definição 2.7.2 Uma seqüência de Cauchy fraca em um espaço normado real ou complexo $B$ é uma seqüência $\left(x_{n}\right)$ em $B$ tal que, para todo $x^{*} \in B^{*}$, a seqüência $\left(x^{*} x_{n}\right)$ é de Cauchy em $\mathbb{R}$ ou $\mathbb{C}$, respectivamente. 
Observação: Uma vez que $\mathbb{R}$ ou $\mathbb{C}$ são espaços completos, então $\lim _{n \rightarrow \infty} x^{*} x_{n}$ existe para todo $x^{*} \in B^{*}$.

Definição 2.7.3 Um espaço normado $B$ é fracamente completo, se cada seqüência de Cauchy fraca em B converge fracamente (ver Definição 2.1.6) em B.

\section{Propriedades:}

1. Um espaço reflexivo é fracamente completo (ver [14], pág. 69, Corolário 29).

2. (Teorema de Alaoglu) A bola fechada unitária no espaço dual $X^{*}$ do espaço de Banach $\mathrm{X}$ dada por $\bar{B}_{1}^{*}=\left\{x^{*} \in X^{*}:\left\|x^{*}\right\| \leqslant 1\right\}$ é compacta na topologia fraca* (ver [14], pág. 424, Teorema 2).

3. (Corolário) Seja X um espaço de Banach reflexivo. Um subconjunto de X é fracamente compacto se, e somente se, é fechado na topologia fraca e limitado na topologia da norma (para definição de topologia da norma, ver [14], pág. 59).

4. Um espaço de Banach é reflexivo se, e somente se, a bola fechada unitária (daí toda bola fechada) é fracamente compacta (ver [14], pág. 425, Teorema 7).

5. (Teorema de Eberlein-S̃mulian) Seja $A$ um subconjunto de um espaço de Banach X. Então as seguintes afirmações são equivalentes:

(i) $A$ é fracamente seqüencialmente compacto, isto é, qualquer seqüência em $A$ tem uma subseqüência que converge fracamente para um elemento de $X$;

(ii) qualquer subconjunto infinito de $A$ tem um ponto limite fraco em $X$;

(iii) o fecho de $A$ na topologia fraca é fracamente compacto, isto é, $A$ é relativamente fracamente compacto (ver [14], pág. 430, Teorema 1).

6. Seja $x_{0}$ um elemento de um espaço de Banach $X$ com a propriedade $\left|x^{*} x_{0}\right| \leqslant k$ para todo $x^{*} \in X^{*},\left\|x^{*}\right\|=1$. Então $\left\|x_{0}\right\| \leqslant k$ (conseqüência imediata de [14], pág. 65, Corolário 14 - Teorema de Hahn-Banach).

Teorema 2.7.4 Seja $X$ um espaço de Banach reflexivo e consideremos $\left(x_{n}\right)_{n \in \mathbb{N}^{*}}$ uma seqüência de elementos de $X$, limitada em norma, isto é, existe um número $k \geqslant 0$ 
para o qual $\left\|x_{n}\right\| \leqslant k$ para todo $n$. Então, existe uma subseqüência $\left(x_{n_{k}}\right)_{k \in \mathbb{N}^{*}} d a$ seqüência original que converge fracamente para um elemento de $X$.

Demonstração: Seja $\bar{B}_{k}(0)$ a bola fechada com centro na origem e raio $k$. Por hipótese, $x_{n} \in \bar{B}_{k}(0)$ para todo $n$. Como $X$ é reflexivo, então, pela Propriedade $4, \bar{B}_{k}(0)$ é fracamente compacta. Logo, pela Propriedade 5 , a seqüência $\left(x_{n}\right)_{n \in \mathbb{N}^{*}}$ possui uma subseqüência que converge fracamente para um elemento de $X$.

Teorema 2.7.5 Sejam X um espaço de Banach reflexivo e $a, b>0$ constantes reais dadas. Consideremos o "cilindro"

$$
P=\left\{(t, x): \quad t_{0} \leqslant t \leqslant t_{0}+a, \quad\left\|x-x_{0}\right\| \leqslant b\right\} \subset \mathbb{R} \times X
$$

onde $t_{0}$ é um número real fixado e $x_{0} \in X$ um elemento fixado. Seja $f(t, x)$ uma função definida sobre $P$, com valores em $X$. Suponhamos que $f(t, x)$ seja fracamentefracamente continua sobre $P$. Então, existe um limite superior $M$ tal que

$$
\|f(t, x)\| \leqslant M, \quad \text { para todo }(t, x) \in P
$$

Demonstração: O conjunto $E_{1}=\left\{t \in \mathbb{R}: t_{0} \leqslant t \leqslant t_{0}+a\right\}$ é compacto na topologia real e $E_{2}=\left\{x \in X:\left\|x-x_{0}\right\| \leqslant b\right\}$ é compacto na topologia fraca, pela Propriedade 3. Assim, pelo Teorema de Tychonoff (ver [14], pág. 32, Teorema 5), $P=E_{1} \times E_{2}$ é compacto na topologia produto "real $\times$ fraca". Como $f(t, x)$ é fracamente-fracamente contínua, então a imagem $f(P)$ é fracamente compacta, e uma vez que $f(P) \subset X$, novamente pela Propriedade 3 , a imagem $f(P)$ é limitada em norma.

Seja $x(t)$ uma função a valores reais, com $x(t) \in X$. A seguir, veremos alguns resultados da teoria de tais funções.

Definição 2.7.6 Sejam $J$ um intervalo sobre o eixo real e $x_{n}(t) \in X$ para $t \in J$, $n=1,2, \ldots$ A seqüência $\left(x_{n}(t)\right)_{n \in \mathbb{N}^{*}}$ converge uniformemente fracamente para $a$ função $x(t)$, se para todo $x^{*} \in X^{*}$ e todo $\epsilon>0$, existe um $n_{0}=n_{0}\left(x^{*}, \epsilon\right)$ tal que $n \geqslant n_{0}$ implica $\left|x^{*} x_{n}(t)-x^{*} x(t)\right|<\epsilon$ para todo $t \in J$.

Teorema 2.7.7 Suponhamos que todos os termos da seqüência $\left(x_{n}(t)\right)_{n \in \mathbb{N}^{*}}$ sejam fracamente contínuos em $J$ (ver Definição 2.1.7) e que a seqüência convirja uniformemente fracamente para a função $x(t)$, então $x(t)$ é fracamente contínua. 
Demonstração: Sejam $x^{*} \in X^{*}$ e $\epsilon>0$ arbitrário. Uma vez que $\left(x_{n}(t)\right)_{n \in \mathbb{N}^{*}}$ converge uniformemente fracamente para a função $x(t)$, então existe $n_{0}=n_{0}\left(x^{*}, \frac{\epsilon}{3}\right)$ tal que para $n \geqslant n_{0}$, temos $\left|x^{*} x_{n}(t)-x^{*} x(t)\right|<\frac{\epsilon}{3}$ para todo $t \in J$. Agora, tomemos $n \geqslant n_{0}$ um inteiro fixado, $t_{0} \in J$ arbitrário, e o fixemos também. A função $x^{*} x_{n}(t)$ é contínua em $t_{0}$, daí, dado $\frac{\epsilon}{3}>0$ podemos escolher $\delta>0$ tal que, para $\left|t-t_{0}\right|<\delta$, temos $\left|x^{*} x_{n}(t)-x^{*} x_{n}\left(t_{0}\right)\right|<\frac{\epsilon}{3}$. Assim, para todo $x^{*} \in X^{*}$ e $\left|t-t_{0}\right|<\delta$, obtemos

$$
\begin{aligned}
& \left|x^{*} x(t)-x^{*} x\left(t_{0}\right)\right| \leqslant\left|x^{*} x(t)-x^{*} x_{n}(t)\right|+\left|x^{*} x_{n}(t)-x^{*} x_{n}\left(t_{0}\right)\right| \\
& +\left|x^{*} x_{n}\left(t_{0}\right)-x^{*} x\left(t_{0}\right)\right|<\frac{\epsilon}{3}+\frac{\epsilon}{3}+\frac{\epsilon}{3}=\epsilon .
\end{aligned}
$$

Portanto $x(t)$ é fracamente contínua.

Definição 2.7.8 Uma seqüência $\left(x_{n}(t)\right)_{n \in \mathbb{N}^{*}}$, definida sobre o intervalo real $J$ é fracamente uniformemente de Cauchy, se para todo $x^{*} \in X^{*}$ e $\epsilon>0$, existe um $n_{0}=n_{0}\left(x^{*}, \epsilon\right)$ tal que $\left|x^{*} x_{n}(t)-x^{*} x_{m}(t)\right|<\epsilon$ para todo $t \in J$ e $m, n \geqslant n_{0}$.

Observação: Toda seqüência que converge uniformemente fracamente é uma seqüência fracamente uniformemente de Cauchy.

Teorema 2.7.9 Sejam X um espaço de Banach fracamente completo e $\left(x_{n}(t)\right)_{n \in \mathbb{N}^{*}}$ uma seqüência fracamente de Cauchy em $J$. Então a seqüência converge uniformemente fracamente para uma função $x(t)$.

Demonstração: Para todo $t$ fixado, a seqüência $\left(x_{n}(t)\right)_{n \in \mathbb{N}^{*}}$ é fracamente de Cauchy, daí esta tem um limite fraco. Denotemos este limite por $x(t)$. Mostremos, então, que $\left(x_{n}(t)\right)_{n \in \mathbb{N}^{*}}$ converge uniformemente fracamente para $x(t)$. Seja $x^{*} \in X^{*}$. Dado $\epsilon>0$ arbitrário, como $\left(x_{n}(t)\right)_{n \in \mathbb{N}^{*}}$ é fracamente de Cauchy, existe um $n_{0}=n_{0}\left(x^{*}, \frac{\epsilon}{2}\right)$ tal que para $n, m \geqslant n_{0}$, temos $\left|x^{*} x_{n}(t)-x^{*} x_{m}(t)\right|<\frac{\epsilon}{2}$ para todo $t \in J$. Fixemos, agora, $t$ e $m$, enquanto $n \rightarrow \infty$, então $\left|x^{*} x_{n}(t)-x^{*} x(t)\right|<\frac{\epsilon}{2}$. Assim,

$$
\left|x^{*} x(t)-x^{*} x_{m}(t)\right| \leqslant\left|x^{*} x(t)-x^{*} x_{n}(t)\right|+\left|x^{*} x_{n}(t)-x^{*} x_{m}(t)\right|<\frac{\epsilon}{2}+\frac{\epsilon}{2}=\epsilon .
$$

Teorema 2.7.10 Sejam X um espaço de Banach fracamente completo e $\left(x_{n}(t)\right)_{n \in \mathbb{N}^{*}}$ uma seqüência fracamente uniformemente de Cauchy de funções fracamente contínuas definidas sobre o intevalo real J. Então a seqüência converge uniformemente fracamente para uma função fracamente contínua $x(t)$, definida sobre $J$. 
Demonstração: A prova é uma conseqüência direta dos Teorema 2.7.7 e 2.7.9.

Definição 2.7.11 Sejam $X$ um espaço de Banach, $\mathscr{F}=\left\{x_{\alpha}(t): \alpha \in C\right\}$ uma família de funções definidas sobre o intervalo real $J$ e $C$ um conjunto de índices. Dizemos que a famíia $\mathscr{F}$ é eqüicontínua, se para cada $t^{\prime} \in J$ e $\epsilon>0$, existe um $\delta=\delta\left(\epsilon, t^{\prime}\right)>0$ tal que $\left|t-t^{\prime}\right|<\delta$ implica $\left\|x_{\alpha}(t)-x_{\alpha}\left(t^{\prime}\right)\right\|<\epsilon$, para todo $\alpha \in C$.

Observação: A eqüicontinuidade da família $\mathscr{F}$ implica a continuidade fraca das $x_{\alpha}(t)$ 's em $J$.

Teorema 2.7.12 Seja $X$ um espaço de Banach reflexivo (daí fracamente completo). Consideremos $\mathscr{F}$, como na Definição 2.7.11, uma família de funções eqüicontínuas e limitadas, isto é, existe um $k \geqslant 0$ tal que $\left\|x_{\alpha}(t)\right\| \leqslant k$ para todo $\alpha \in C$ e $t \in$ $J$. Então existe uma seqüência $\alpha(n)$ tal que a seqüência $\left(x_{\alpha(n)}\right)_{n \in \mathbb{N}^{*}}$ é fracamente uniformemente de Cauchy, logo converge uniformemente fracamente.

Demonstração: Seja $\left\{t_{n}: n=1,2, \ldots\right\}$ um conjunto denso enumerável em $J$. Por hipótese, o conjunto $\left\{x_{\alpha}\left(t_{1}\right): \alpha \in C\right\}$ é limitado. Daí, pelo Teorema 2.7.4, existe uma seqüência $\alpha_{11}, \alpha_{12}, \ldots, \alpha_{1 n}, \ldots \in C$ tal que $\left(x_{\alpha_{1 n}}\left(t_{1}\right)\right)_{n \in \mathbb{N}^{*}}$ é fracamente convergente, logo fracamente de Cauchy. Agora, consideremos o conjunto limitado $\left\{x_{\alpha_{1 n}}\left(t_{2}\right): n=1,2, \ldots\right\}$. Este contém uma subseqüência fracamente de Cauchy, que denotaremos por $x_{\alpha_{21}}\left(t_{2}\right), x_{\alpha_{22}}\left(t_{2}\right), \ldots, x_{\alpha_{2 n}}\left(t_{2}\right), \ldots$ Assim, sucessivamente, construiremos a seqüência $\left(x_{\alpha_{i j}}(t)\right)$ para todo $i$, e tomamos $\alpha(n)=\alpha_{n n}$. Então a subseqüência $\left(x_{\alpha(n)}(t)\right)_{n \in \mathbb{N}^{*}}$ é fracamente de Cauchy para todo $t=t_{1}, t_{2}, \ldots$. Mostraremos, agora, que $\left(x_{\alpha(n)}(t)\right)_{n \in \mathbb{N}^{*}}$ é fracamente uniformemente de Cauchy. Para este fim, sejam $x^{*} \in X^{*}$ e $\epsilon>0$ arbitrário. Sem perda de generalidade, podemos supor que $\left\|x^{*}\right\|=1$. Escolhemos $\delta>0$ para $\frac{\epsilon}{3}$ dado, por meio da eqüicontinuidade. Os intervalos de raio $\delta$ e centro em $t_{i}(i=1,2, \ldots)$ cobrem $J$. Daí existe uma subcobertura finita.

Suponhamos que a subcobertura finita consista $\operatorname{dos} N$ primeiros intervalos. Como $\left(x_{\alpha(n)}(t)\right)_{n \in \mathbb{N}^{*}}$ é fracamente de Cauchy em $t_{1}, t_{2}, \ldots t_{N}$, podemos escolher $n_{0}$ tal que para $k_{1}, k_{2} \geqslant n_{0}$, temos

$$
\left|x^{*} x_{\alpha\left(k_{1}\right)}\left(t_{i}\right)-x^{*} x_{\alpha\left(k_{2}\right)}\left(t_{i}\right)\right|<\frac{\epsilon}{3}, \quad \text { para } i=1,2, \ldots, N .
$$

Sejam $k_{1}, k_{2} \geqslant n_{0}$ e $t \in J$ arbitrário. Existe um termo da subcobertura finita contendo $t$. Podemos supor, sem perda de generalidade, que este é o primeiro intervalo. 
Logo,

$$
\begin{aligned}
& x^{*} x_{\alpha\left(k_{1}\right)}(t)-x^{*} x_{\alpha\left(k_{2}\right)}(t)=x^{*} x_{\alpha\left(k_{1}\right)}(t)-x^{*} x_{\alpha\left(k_{1}\right)}\left(t_{1}\right) \\
& +x^{*} x_{\alpha\left(k_{1}\right)}\left(t_{1}\right)-x^{*} x_{\alpha\left(k_{2}\right)}\left(t_{1}\right)+x^{*} x_{\alpha\left(k_{2}\right)}\left(t_{1}\right)-x^{*} x_{\alpha\left(k_{2}\right)}(t) .
\end{aligned}
$$

Para $l=1,2$, uma vez que temos eqüicontinuidade, então

$$
\left|x^{*} x_{\alpha\left(k_{l}\right)}(t)-x^{*} x_{\alpha\left(k_{l}\right)}\left(t_{1}\right)\right| \leqslant\left\|x^{*}\right\|\left\|x_{\alpha\left(k_{l}\right)}(t)-x_{\alpha\left(k_{l}\right)}\left(t_{1}\right)\right\| \leqslant 1 \cdot \frac{\epsilon}{3}=\frac{\epsilon}{3} .
$$

E ainda de (2.79), temos

$$
\left|x^{*} x_{\alpha\left(k_{1}\right)}\left(t_{1}\right)-x^{*} x_{\alpha\left(k_{2}\right)}\left(t_{1}\right)\right|<\frac{\epsilon}{3} .
$$

Retornando agora à equação (2.80), obtemos

$$
\left|x^{*} x_{\alpha\left(k_{1}\right)}(t)-x^{*} x_{\alpha\left(k_{2}\right)}(t)\right|<\frac{\epsilon}{3}+\frac{\epsilon}{3}+\frac{\epsilon}{3}=\epsilon .
$$

Como $n_{0}$ não depende de $t$, a prova está completa.

\subsubsection{O Teorema de Existência}

Veremos agora que, considerando o problema de valor inicial (2.1) em um espaço de Banach reflexivo, com $f(t, x)$ uma função fracamente-fracamente contínua sobre o "cilindro" dado por (2.78), é possível obtermos soluções fracas para (2.1). Inicialmente, pelo Teorema 2.7.5, existe um limite superior $M>0$ da função $f(t, x)$ em norma. Então, tomemos $\alpha=\min \left\{a, \frac{b}{M}\right\}$.

Teorema 2.7.13 O problema de valor inicial (2.1) possui pelo menos uma solução fraca definida sobre $\left[t_{0}, t_{0}+\alpha\right]$.

Demonstração: O problema de valor inicial (2.1) é equivalente à equação integral

$$
x^{*} x(t)=x^{*} x_{0}+\int_{t_{0}}^{t} x^{*} f(s, x(s)) d s, \quad \text { para todo } x^{*} \in X^{*} .
$$

Logo, para encontrarmos uma solução fraca de (2.1), basta encontrarmos uma solução para a equação integral (2.81), como vimos no Teorema 2.1.27.

Sejam $\delta>0$ uma constante real apropriada e $x_{0}(t)$ uma função fracamente continuamente diferenciável sobre $\left[t_{0}-\delta, t_{0}\right]$, isto é, $x_{0}(t)$ é fracamente diferenciável 
(ver Definição 2.1.8) e sua derivada, como uma função de $t$, é fracamente contínua. E ainda, $x_{0}(t)$ satisfaz as seguintes propriedades: $x_{0}\left(t_{0}\right)=x_{0}, x_{0}^{\prime}\left(t_{0}\right)=f\left(t_{0}, x_{0}\right)$, $\left\|x_{0}(t)-x_{0}\right\| \leqslant b$ e $\left\|x_{0}^{\prime}(t)\right\| \leqslant M$.

Observemos que para $\delta>0$ suficientemente pequeno, a função

$$
x_{0}(t)=x_{0}+\left(t-t_{0}\right) f\left(t_{0}, x_{0}\right)
$$

tem as propriedades exigidas acima, mas não nos restringiremos à escolha de $x_{0}(t)$ além das propriedades exigidas. Para $0<\epsilon \leqslant \delta$, definimos $x_{\epsilon}(t)$ sobre $\left[t_{0}-\delta, t_{0}+\alpha[\right.$ por

$$
x_{\epsilon}(t)= \begin{cases}x_{0}(t), & \text { para } t_{0}-\delta \leqslant t \leqslant t_{0} \\ x_{0}+\int_{t_{0}}^{t} f\left(s, x_{\epsilon}(s-\epsilon)\right) d s, & \text { para } t_{0} \leqslant t \leqslant t_{0}+\alpha .\end{cases}
$$

Esta fórmula define $x_{\epsilon}(t)$ primeiro no intervalo $\left[t_{0}, t_{0}+\alpha_{1}\right]$, onde $\alpha_{1}=\min \{\alpha, \epsilon\}$. A substituição é admitida, $f\left(s, x_{\epsilon}(s-\epsilon)\right)$ é uma função fracamente contínua em $s$, logo é fracamente integrável. Seja $x^{*} \in X^{*}$ arbitrário e suponhamos, sem perda de generalidade, que $\left\|x^{*}\right\|=1$. Para $t_{0} \leqslant t \leqslant t_{0}+\alpha_{1}$, temos

$$
x^{*}\left(x_{\epsilon}(t)-x_{0}\right)=\int_{t_{0}}^{t} x^{*} f\left(s, x_{\epsilon}(s-\epsilon)\right) d s
$$

Então,

$$
\left|x^{*}\left(x_{\epsilon}(t)-x_{0}\right)\right| \leqslant M\left|t-t_{0}\right| \leqslant M \frac{b}{M}=b .
$$

Assim, da Propriedade 6, segue que $\left\|x_{\epsilon}(t)-x_{0}\right\| \leqslant b$ para $t_{0} \leqslant t \leqslant t_{0}+\alpha_{1}$. Daí, $x_{\epsilon}(t)$ pode ser estendida para o intervalo $\left[t_{0}-\delta, t_{0}+\alpha_{2}\right]$, onde $\alpha_{2}=\min \{\alpha, 2 \epsilon\}$. Estendemos a definição de $x_{\epsilon}(t)$ da mesma maneira para $\left[t_{0}-\delta, t_{0}+\alpha\right]$, conforme a relação $\left\|x_{\epsilon}(t)-x_{0}\right\| \leqslant b$. Então, a família de funções $\left\{x_{\epsilon}(t)\right\}$, com $0<\epsilon \leqslant \delta$ é limitada. Mostremos agora que a família $\left\{x_{\epsilon}(t)\right\}$ é eqüicontínua no intervalo fechado $\left[t_{0}-\delta, t_{0}+\alpha\right]$. De fato, seja $x^{*} \in X^{*}$ arbitrário, com $\left\|x^{*}\right\|=1$. Então para quaisquer $t_{1}, t_{2} \in\left[t_{0}, t_{0}+\alpha\right]$

$$
\left|x^{*} x_{\epsilon}\left(t_{1}\right)-x^{*} x_{\epsilon}\left(t_{2}\right)\right| \leqslant \int_{t_{1}}^{t_{2}}\left\|f\left(s, x_{\epsilon}(s-\epsilon)\right)\right\| d s \leqslant M\left|t_{1}-t_{2}\right| .
$$

Para $t_{1}, t_{2} \in\left[t_{0}-\delta, t_{0}\right]$, temos

$$
\left|x^{*} x_{\epsilon}\left(t_{1}\right)-x^{*} x_{\epsilon}\left(t_{2}\right)\right| \leqslant\left\|x_{0}\left(t_{1}\right)-x_{0}\left(t_{2}\right)\right\|=\left\|f\left(t_{0}, x_{0}\right)\left(t_{1}-t_{2}\right)\right\| \leqslant M\left|t_{1}-t_{2}\right| .
$$


Em ambos os casos, segue da Propriedade 6 que

$$
\left\|x_{\epsilon}\left(t_{1}\right)-x_{\epsilon}\left(t_{2}\right)\right\| \leqslant M\left|t_{1}-t_{2}\right|
$$

Assim, dado $\eta>0$ tomemos $\delta=\frac{\eta}{2 M}$, tal que $\left|t_{1}-t_{2}\right|<\delta$. Logo

$$
\left\|x_{\epsilon}\left(t_{1}\right)-x_{\epsilon}\left(t_{2}\right)\right\| \leqslant M \frac{\eta}{2 M}<\eta .
$$

Portanto, a família $\left\{x_{\epsilon}(t)\right\}$, com $0<\epsilon \leqslant \delta$, é eqüicontínua. Agora, pelo Teorema 2.7.12, existe uma seqüência $\epsilon(1), \epsilon(2), \ldots$, tal que quando $n \rightarrow \infty, \epsilon(n) \rightarrow 0$, e $\left(x_{\epsilon(n)}(t)\right)$ é fracamente uniformemente de Cauchy sobre $\left[t_{0}-\delta, t_{0}+\alpha\right]$. Então converge uniformemente fracamente. Pelo Teorema 2.7.10, existe uma função $x(t)$ fracamente contínua tal que $\left(x_{\epsilon(n)}(t)\right)$ converge uniformemente fracamente para $x(t)$, quando $n \rightarrow \infty$. Como $X$ é reflexivo, então todas as bolas fechadas em $X$ são fracamente compactas, mais especificamente, fracamente fechadas. Uma vez que $\left\|x_{\epsilon(n)}(t)-x_{0}\right\| \leqslant b$ e $x^{*} x_{\epsilon(n)}(t) \rightarrow x^{*} x(t)$ para todo $x^{*} \in X^{*}$, então para todo $t$, $\left\|x(t)-x_{0}\right\| \leqslant b$. Daí, $f(s, x(s))$ existe e é fracamente contínua. Logo, para todo $x^{*} \in X^{*}, t_{0} \leqslant t \leqslant t_{0}+\alpha$ arbitrário e $n \rightarrow \infty$, temos

$$
x^{*} f\left(s, x_{\epsilon(n)}(s-\epsilon(n))\right) \rightarrow x^{*} f(s, x(s)) .
$$

Fixando $x^{*}$ e $s$, segue que $x^{*} f\left(s, x_{\epsilon(n)}(s-\epsilon(n))\right)$ pode ser majorada por uma constante universal, pois $\left\|x^{*}\right\|=1$ e $\left\|f\left(s, x_{\epsilon(n)}(s-\epsilon(n))\right)\right\| \leqslant M$. Logo, pelo Teorema da Convergência Dominada, $x(t)$ satisfaz a equação integral (2.81). Portanto, $x(t)$ é uma solução do problema (2.1).

\section{Observações:}

1. Soluções fracas são sempre uniformemente contínuas, uma vez que pela definição de solução fraca, $x$ é uma função $A C$ (ver observação da Definição A.1.8).

2. Como vimos no Contra-Exemplo 2, não existe uma solução clássica para o problema (2.18) satisfazendo $x(0)=\odot$. Entretanto, como todo espaço de Hilbert é reflexivo, pelo Teorema 2.7.13, existe pelo menos uma solução fraca para o problema (2.18) satisfazendo $x(0)=\odot$. 


\section{Estabilidade e Instabilidade por}

\section{Primeira Aproximação Linear}

Consideremos, em um espaço de Banach $X$, com a norma $\|\cdot\|$, a equação diferencial não linear da forma

$$
\frac{d x}{d t}=f(t, x), \quad t_{0} \leqslant t<\infty
$$

onde $f:\left[t_{0}, \infty[\times X \longrightarrow X\right.$ é uma função contínua. Vimos no Capítulo 2 o conceito de dependência contínua sobre o dado inicial $\left(t_{0}, x_{0}\right)$. Embora este conceito seja importante, somente fornece informações sobre intervalos de tempo finito. Um conceito mais importante é o de dependência contínua sobre o dado inicial $\left(t_{0}, x_{0}\right)$ sobre intervalos de tempo infinito, e este é o conceito de estabilidade que veremos neste capítulo. Estudaremos estabilidade e instabilidade, por primeira aproximação linear, das soluções da equação diferencial (3.1). Para este estudo, utilizaremos [7] e alguns conceitos que se encontram nos Apêndices A.2 e A.3, como por exemplo: espectro, resolvente de um operador linear (Definição A.2.1) e exponencial de um operador linear limitado (equação (A.2)).

Inicialmente, afirmamos que a investigação da estabilidade de uma solução $x=$ $\phi(t)$ da equação (3.1) sempre pode ser reduzida à investigação da estabilidade da solução nula de uma equação auxiliar. De fato, tomando $y(t)=x(t)-\phi(t)$, onde $x(t)$ e $\phi(t)$ são soluções de (3.1), obtemos

$$
\frac{d y}{d t}=f(t, x(t))-f(t, \phi(t))=f(t, y(t)+\phi(t))-f(t, \phi(t)) .
$$


Definindo $g(t, y)=f(t, y(t)+\phi(t))-f(t, \phi(t))$, obtemos a seguinte equação

$$
\frac{d y}{d t}=g(t, y), \quad t_{0} \leqslant t<\infty
$$

onde $g(t, 0)=0$. Daí, a solução $x=\phi(t)$ da equação (3.1) é levada sobre a solução nula $y \equiv 0$ da equação (3.2), enquanto qualquer outra solução $x=\psi(t)$ é levada na diferença $y=\phi(t)-\psi(t)$.

Podemos reduzir a equação (3.2) a uma forma mais conveniente para o estudo da estabilidade, se a função $f(t, x)$ for continuamente Fréchet diferenciável em relação à segunda variável em uma vizinhança do ponto $\phi(t)$. Do Teorema de Taylor (ver [23], pág. 10, ou [30], pág. 187, Teorema 6.1), obtemos

$$
\begin{array}{r}
f(t, x(t))=f(t, \phi(t))+d_{x} f(t, \phi(t))(x(t)-\phi(t))+F(t, x(t)-\phi(t)) \\
\Longrightarrow g(t, y)=f(t, y(t)+\phi(t))-f(t, \phi(t))=d_{x} f(t, \phi(t)) y(t)+F(t, y(t)),
\end{array}
$$

onde $\lim _{y(t) \rightarrow 0} \frac{F(t, y(t))}{\|y(t)\|}=0$, ou ainda, $\|F(t, y)\| \leqslant c_{t}(y)\|y\|$, com $\lim _{y \rightarrow 0} c_{t}(y)=0$, e $d_{x} f(t, \phi(t))$ é um operador linear limitado sobre $X$. Se a convergência for uniforme em $t$, isto é, $\|F(t, y)\| \leqslant c(y)\|y\|$, com $\lim _{y \rightarrow 0} c(y)=0$, então para qualquer $q>0$, existe um $\eta>0$ tal que

$$
\|F(t, y)\| \leqslant q\|y\|, \quad \text { para } y \text { na bola } \bar{B}_{\eta}=\{y \in X:\|y\| \leqslant \eta\} .
$$

Resultados análogos também podem ser obtidos quando $f(t, x)$ é Gâteaux ou Hadamard diferenciável na variável $x$ (ver [30], pág. 192, Teorema 6.5). Portanto, segue de (3.3) que podemos reescrever a equação (3.2) da seguinte forma

$$
\frac{d y}{d t}=A y+F(t, y), \quad t_{0} \leqslant t<\infty
$$

onde $A=d_{x} f(t, \phi(t))$ é um operador linear limitado sobre $X$.

A equação linear homogênea associada a (3.5) dada por

$$
\frac{d y}{d t}=A y, \quad t_{0} \leqslant t<\infty
$$

desempenha um importante papel na investigação da estabilidade da equação não linear (3.5). Mostraremos que uma pequena perturbação da equação (3.6) não poderá produzir uma grande mudança no comportamento das soluções da mesma. Veremos que, sob certas condições, a questão da estabilidade da solução nula da equação (3.5), com uma pequena perturbação $F(t, y)$, pode ser resolvida pela localização do espectro $\sigma(A)$ (ver Definição A.2.1) do operador linear limitado $A$. Entretanto, veremos que se $f(t, x)$ for apenas Hadamard diferenciável, este resultado não é verdadeiro. 


\subsection{Estabilidade e Instabilidade das Soluções da Equação Não Linear}

Consideremos a equação diferencial (3.1) e suponhamos que a função $f(t, x)$ seja continuamente Fréchet diferenciável em $x$ e satisfaça condições suficientes para assegurar existência, unicidade e dependência contínua em relação ao dado inicial $\left(t_{0}, x_{0}\right)$. Inicialmente temos a seguinte definição:

Definição 3.1.1 Seja $\phi(t)$ uma solução da equação (3.1) definida para todo $t \geqslant$ $t_{0}$. Dizemos que $\phi(t)$ é estável, se para todo $\epsilon>0$, existe um $\delta>0$ tal que se $\psi(t)$ é solução de (3.1) e $\left\|\phi\left(t_{0}\right)-\psi\left(t_{0}\right)\right\|<\delta$, então $\psi(t)$ está definida para todo $t \geqslant t_{0}$ e satisfaz a desigualdade $\|\phi(t)-\psi(t)\|<\epsilon$ para todo $t \geqslant t_{0}$. Dizemos que $\phi(t)$ é uniformemente estável, se a constante $\delta$ pode ser escolhida independente de $t_{0}$. A solução $\phi(t)$ é instável se não é estável. Finalmente, dizemos que $\phi(t)$ é assintoticamente estável, se é estável e existe um $\delta_{0}>0$ tal que $\left\|\phi\left(t_{0}\right)-\psi\left(t_{0}\right)\right\|<\delta_{0}$ implica $\lim _{t \rightarrow \infty}\|\phi(t)-\psi(t)\|=0$.

Como observamos anteriormente, deduziremos resultados sobre a estabilidade da equação não linear (3.5) a partir da localização do espectro $\sigma(A)$. Então, inicialmente, como $A$ é um operador linear limitado, segue de (A.4) que uma solução de (3.6) satisfazendo a condição inicial $x\left(t_{0}\right)=x_{0}$ é dada por

$$
x(t)=e^{A\left(t-t_{0}\right)} x_{0} .
$$

E ainda, esta solução é única. De fato, consideremos a equação $y(t)=e^{-A\left(t-t_{0}\right)} x(t)$, onde $x(t)$ é uma solução da equação (3.6), satisfazendo $x\left(t_{0}\right)=x_{0}$. Então $t \longmapsto y(t)$ também é continuamente diferenciável sobre $] t_{0}, \infty[$, e por (A.4), temos

$$
\frac{d y(t)}{d t}=-A e^{-A\left(t-t_{0}\right)} x(t)+e^{-A\left(t-t_{0}\right)} \frac{d x(t)}{d t}=-A e^{-A\left(t-t_{0}\right)} x(t)+e^{-A\left(t-t_{0}\right)} A x(t)=0 .
$$

Portanto, $y(t)$ é constante, mas $y\left(t_{0}\right)=x\left(t_{0}\right)=x_{0}$, logo $y(t) \equiv x_{0}$. Assim, $x(t)=$ $e^{A\left(t-t_{0}\right)} x_{0}$

O lema a seguir é uma conseqüência do Teorema da Aplicação Espectral (ver [26], pág. 381, Teorema 7.4-2). O mesmo pode ser encontrado em [7], pág. 19, Lema 2.2. 
Lema 3.1.2 Se $\left\|e^{A}\right\| \leqslant q$, então o espectro $\sigma(A)$ de $A$ está localizado no semi-plano fechado $\Re(\lambda) \leqslant \ln q$.

Teorema 3.1.3 Seja $\alpha$ um número real. Se existe $N_{\alpha}>0$ tal que

$$
\left\|e^{A t}\right\| \leqslant N_{\alpha} e^{\alpha t}, \quad t \geqslant 0
$$

então $\Re(\lambda) \leqslant \alpha$ para todo $\lambda \in \sigma(A)$. Reciprocamente, se $\Re(\lambda)<\alpha$ para todo $\lambda \in \sigma(A)$, então existe $N_{\alpha}>0$ tal que (3.8) é satisfeita.

Demonstração: Suponhamos que a estimativa (3.8) seja satisfeita. Então, pelo Lema 3.1.2, o espectro $\sigma(A t)$ está localizado no semi-plano $\Re(\lambda) \leqslant \ln N_{\alpha}+\alpha t, t \geqslant 0$. Mas, pelo Teorema da Aplicação Espectral, $\sigma(A t)=t \sigma(A)$, daí para qualquer $t>0 \mathrm{o}$ espectro $\sigma(A)$ está localizado no semi-plano $\Re(\lambda) \leqslant \frac{\ln N_{\alpha}}{t}+\alpha$. Uma vez que $t>0$ é arbitrário, então $\Re(\lambda) \leqslant \alpha$, e a primeira parte da afirmação está provada.

Para provarmos a segunda parte, consideremos $e^{A t}$ definida em (A.3) por

$$
e^{A t}=\frac{1}{2 \pi \imath} \int_{\Gamma_{A}} e^{\lambda t} R(\lambda: A) d \lambda
$$

onde $R(\lambda: A)=(\lambda I-A)^{-1}$, o contorno $\Gamma_{A}$ é percorrido no sentido positivo e está contido completamente no semi-plano $\Re(\lambda)<\alpha$. Então

$$
\left\|e^{A t}\right\| \leqslant \frac{1}{2 \pi} \int_{\Gamma_{A}}\left|e^{\lambda t}\right|\|R(\lambda: A)\||d \lambda|=\frac{1}{2 \pi} \int_{\Gamma_{A}} e^{\Re(\lambda) t}\|R(\lambda: A)\||d \lambda| \leqslant N_{\alpha} e^{\alpha t},
$$

onde $N_{\alpha}=\left(\frac{l}{2 \pi}\right) \max _{\lambda \in \Gamma_{A}}\|R(\lambda: A)\|$ e $l$ é o comprimento do contorno $\Gamma_{A}$.

O comportamento no infinito das soluções da equação linear (3.6), com a condição inicial $x\left(t_{0}\right)=x_{0}$, depende essencialmente da localização do espectro de $A$.

Suponhamos que o espectro $\sigma(A)$ esteja localizado no interior do semi-plano esquerdo. Então para qualquer $\alpha>0$ tal que $\Re(\lambda)<-\alpha$ para todo $\lambda \in \sigma(A)$, segue do Teorema 3.1 .3 que existe um $N_{\alpha}>0$ tal que $\left\|e^{A\left(t-t_{0}\right)}\right\| \leqslant N_{\alpha} e^{-\alpha\left(t-t_{0}\right)}$ para $t \geqslant t_{0}$. Portanto, por (3.7),

$$
\|x(t)\| \leqslant N_{\alpha} e^{-\alpha\left(t-t_{0}\right)}\left\|x\left(t_{0}\right)\right\|, \quad t \geqslant t_{0}
$$

Reciprocamente, se a estimativa (3.9) vale para qualquer solução da equação (3.6), o espectro $\sigma(A)$ está localizado no interior do semi-plano esquerdo. 
A suposição que $\sigma(A)$ está localizado no interior do semi-plano esquerdo nos permite introduzir uma nova norma em $X$, que desempenha um papel crucial na questão da estabilidade. Para todo $x \in X$, definimos

$$
\|x\|_{A}=\int_{0}^{\infty}\left\|e^{A s} x\right\| d s .
$$

Da Desigualdade de Minkowski com $p=1$ (ver [31], pág. 548), temos $\|x+y\|_{A}=\int_{0}^{\infty}\left\|e^{A s} x+e^{A s} y\right\| d s \leqslant \int_{0}^{\infty}\left\|e^{A s} x\right\| d s+\int_{0}^{\infty}\left\|e^{A s} y\right\| d s=\|x\|_{A}+\|y\|_{A}$. As demais exigências da definição de norma seguem facilmente. Logo $\|\cdot\|_{A}$ define uma norma em $X$. Temos ainda o seguinte resultado:

Lema 3.1.4 A norma $\|\cdot\|_{A}$ é equivalente à norma original em $X$ dada por $\|\cdot\|$.

Demonstração: Devemos mostrar que existem constantes positivas $m$ e $M$ tais que $m\|x\| \leqslant\|x\|_{A} \leqslant M\|x\|$, para todo $x \in X$. Temos

$$
\|x\|_{A} \leqslant\|x\| \int_{0}^{\infty}\left\|e^{A s}\right\| d s \leqslant\|x\| N_{\alpha} \int_{0}^{\infty} e^{-\alpha s} d s=\frac{N_{\alpha}}{\alpha}\|x\| .
$$

Por outro lado, uma vez que $\|x\|=\left\|e^{-A s} e^{A s} x\right\| \leqslant\left\|e^{-A s}\right\|\left\|e^{A s} x\right\| \leqslant e^{s\|A\|}\left\|e^{A s} x\right\|$, obtemos

$$
\|x\|_{A}=\int_{0}^{\infty}\left\|e^{A s} x\right\| d s \geqslant\|x\| \int_{0}^{\infty} e^{-s\|A\|} d s=\frac{1}{\|A\|}\|x\| .
$$

Portanto, basta tomarmos $M=\frac{N_{\alpha}}{\alpha}$ e $m=\frac{1}{\|A\|}$.

Na norma dada por (3.10), a solução da equação linear (3.6) decresce monotonicamente quando $t$ cresce, ou ainda, tende monotonicamente a zero quando $t \rightarrow \infty$. De fato, sem perda de generalidade, podemos assumir que $t_{0}=0$ em (3.7). Daí

$$
\|x(t)\|_{A}=\int_{0}^{\infty}\left\|e^{A s} x(t)\right\| d s=\int_{0}^{\infty}\left\|e^{A(t+s)} x_{0}\right\| d s=\int_{t}^{\infty}\left\|e^{A s} x_{0}\right\| d s
$$

e, portanto,

$$
\frac{d}{d t}\|x(t)\|_{A}=-\left\|e^{A t} x_{0}\right\|<0, \quad \text { para } x_{0} \neq 0 .
$$

Com base no que acabamos de ver, temos o seguinte resultado para a equação não linear (3.5): 
Teorema 3.1.5 Suponhamos que o espectro $\sigma(A)$ do operador A esteja localizado no interior do semi-plano esquerdo tal que $\left\|e^{A t}\right\| \leqslant N_{\alpha_{0}} e^{-\alpha_{0} t}$, e suponhamos que a condição (3.4) seja satisfeita para $q<\frac{\alpha_{0}}{N_{\alpha_{0}}}$. Então a solução nula da equação (3.5) é assintoticamente estável.

Demonstração: Seja $x(t)$ uma solução da equação (3.5) satisfazendo a condição $\left\|x\left(t_{0}\right)\right\|<\eta_{0} \leqslant \eta$, onde o número $\eta_{0}$ será escolhido posteriormente, e seja $t_{0} \leqslant$ $t<T$ um intervalo em que $\|x(t)\|<\eta$. Tal intervalo existe devido à continuidade da solução. Mostraremos que $T$ pode ser tomado arbitrariamente grande e ainda $\|x(t)\| \rightarrow 0$ quando $t \rightarrow \infty$.

Consideremos, no espaço de Banach $X$, a norma dada por (3.10). Então, pelo Lema 3.1.4, $m\|x\| \leqslant\|x\|_{A} \leqslant M\|x\|$, onde $m=\frac{1}{\|A\|}$ e $M=\frac{N_{\alpha_{0}}}{\alpha_{0}}$. Tomando $y(t)=$ $e^{-A t} x(t)$, segue de (A.4) e (3.5) que

$$
\frac{d y}{d t}=-A e^{-A t} x+e^{-A t} \frac{d x}{d t}=e^{-A t}\left(\frac{d x}{d t}-A x\right)=e^{-A t} F(t, x),
$$

e ainda,

$$
\|x(t)\|_{A}=\left\|e^{A t} y(t)\right\|_{A}=\int_{0}^{\infty}\left\|e^{A(\tau+t)} y(t)\right\| d \tau=\int_{t}^{\infty}\left\|e^{A \tau} y(t)\right\| d \tau .
$$

De (3.12) obtemos

$$
\begin{aligned}
& \frac{\|x(t+h)\|_{A}-\|x(t)\|_{A}}{h}=\frac{\int_{t+h}^{\infty}\left\|e^{A \tau} y(t+h)\right\| d \tau-\int_{t}^{\infty}\left\|e^{A \tau} y(t)\right\| d \tau}{h} \\
& =\frac{\int_{t+h}^{\infty}\left\|e^{A \tau} y(t+h)\right\| d \tau-\int_{t+h}^{\infty}\left\|e^{A \tau} y(t)\right\| d \tau+\int_{t+h}^{\infty}\left\|e^{A \tau} y(t)\right\| d \tau-\int_{t}^{\infty}\left\|e^{A \tau} y(t)\right\| d \tau}{h} \\
& =-\frac{1}{h} \int_{t}^{t+h}\left\|e^{A \tau} y(t)\right\| d \tau+\int_{t+h}^{\infty} \frac{\left\|e^{A \tau} y(t+h)\right\|-\left\|e^{A \tau} y(t)\right\|}{h} d \tau=I_{1}(h)+I_{2}(h) .
\end{aligned}
$$

Estimaremos $I_{1}(h)$ e $I_{2}(h)$, assumindo que $t_{0} \leqslant t<T$. Inicialmente, da Regra de L'Hospital, temos

$$
\begin{gathered}
\lim _{h \rightarrow 0} I_{1}(h)=-\lim _{h \rightarrow 0} \frac{1}{h} \int_{t}^{t+h}\left\|e^{A \tau} y(t)\right\| d \tau=-\lim _{h \rightarrow 0}\left\|e^{A(t+h)} y(t)\right\|=-\left\|e^{A t} y(t)\right\| \\
\Longrightarrow \lim _{h \rightarrow 0} I_{1}(h)=-\|x(t)\|
\end{gathered}
$$


Agora, tomando $s=\tau-t-h$ em $I_{2}(h)$, obtemos

$$
\begin{aligned}
I_{2}(h) & \leqslant \int_{t+h}^{\infty} \frac{\left\|e^{A \tau}[y(t+h)-y(t)]\right\|}{h} d \tau=\int_{0}^{\infty}\left\|e^{A s} e^{A(t+h)} \frac{y(t+h)-y(t)}{h}\right\| d s \\
& =\left\|e^{A(t+h)} \frac{y(t+h)-y(t)}{h}\right\|_{A} \leqslant M\left\|e^{A h}\right\|\left\|e^{A t} \frac{y(t+h)-y(t)}{h}\right\| .
\end{aligned}
$$

Logo, por (3.4) e (3.11), temos

$$
\varlimsup_{h \rightarrow 0} I_{2}(h) \leqslant M\left\|e^{A t} y^{\prime}(t)\right\|=M\|F(t, x(t))\| \leqslant q M\|x(t)\| .
$$

Portanto, para a primeira derivada da norma $\|x(t)\|_{A}$ dada por

$$
\frac{d^{+}\|x(t)\|_{A}}{d t}=\lim _{h \rightarrow 0^{+}} \frac{\|x(t+h)\|_{A}-\|x(t)\|_{A}}{h}
$$

que existe, uma vez que $x(t)$ é diferenciável, segue de (3.13) e (3.14) que

$$
\frac{d^{+}\|x(t)\|_{A}}{d t} \leqslant(q M-1)\|x(t)\| \leqslant \frac{q M-1}{m}\|x(t)\|_{A}=-\alpha\|x(t)\|_{A},
$$

onde $\alpha=\frac{1-q M}{m}>0$, uma vez que $q M=q \frac{N_{\alpha_{0}}}{\alpha_{0}}<\frac{\alpha_{0}}{N_{\alpha_{0}}} \frac{N_{\alpha_{0}}}{\alpha_{0}}=1$.

Resolvendo a desigualdade em (3.15), obtemos

$$
\|x(t)\|_{A} \leqslant e^{-\alpha(t-\tau)}\|x(\tau)\|_{A}, \quad t_{0} \leqslant \tau \leqslant t<T .
$$

Agora, voltando para norma original,

$$
\|x(t)\| \leqslant N e^{-\alpha(t-\tau)}\|x(\tau)\|, \quad t_{0} \leqslant \tau \leqslant t<T,
$$

onde $N=\frac{M}{m}$.

Então tomamos $\eta_{0}=\min \left\{\eta, \frac{\eta}{N}\right\}$. Desta forma, $T$ pode ser tomado arbitrariamente grande. De fato, se supormos que $\|x(T)\|=\eta$ e aplicarmos o limite em (3.16) para $t \rightarrow T$, com $\tau=t_{0}$, obtemos

$$
\eta=\|x(T)\| \leqslant N e^{-\alpha\left(T-t_{0}\right)}\left\|x\left(t_{0}\right)\right\|<N \eta_{0} e^{-\alpha\left(T-t_{0}\right)}<N \frac{\eta}{N}=\eta,
$$

o que é um absurdo. E ainda da desigualdade (3.16), temos $\|x(t)\| \rightarrow 0$ quando $t \rightarrow \infty$.

Observação: Uma outra maneira de demonstrarmos o Teorema 3.1.5 é utilizando a Fórmula da Variação das Constantes (ver [20], pág. 81, Teorema 1.1) e a Desigualdade de Gronwall (ver [20], pág. 36, Corolário 6.6). De fato, por hipótese, segue 
que para qualquer $q>0$, existe um $\eta>0$ tal que $\|F(t, y)\| \leqslant q\|y\|$ para $\|y\| \leqslant \eta$. Seja $x(t)$ uma solução da equação (3.5) satisfazendo a condição $\left\|x\left(t_{0}\right)\right\|<\frac{\eta}{N_{\alpha_{0}}}$, e seja $t_{0} \leqslant t<T$ um intervalo em que $\|x(t)\|<\eta$. Pela Fórmula da Variação das Constantes, temos

$$
\begin{gathered}
\|x(t)\| \leqslant\left\|e^{A\left(t-t_{0}\right)}\right\|\left\|x\left(t_{0}\right)\right\|+\int_{t_{0}}^{t}\left\|e^{A(t-s)}\right\|\|F(s, x(s))\| d s \\
\Longrightarrow \\
\|x(t)\| \leqslant N_{\alpha_{0}} e^{-\alpha_{0}\left(t-t_{0}\right)}\left\|x\left(t_{0}\right)\right\|+q N_{\alpha_{0}} \int_{t_{0}}^{t} e^{-\alpha_{0}(t-s)}\|x(s)\| d s \\
\Longrightarrow e^{\alpha_{0}\left(t-t_{0}\right)}\|x(t)\| \leqslant N_{\alpha_{0}}\left\|x\left(t_{0}\right)\right\|+q N_{\alpha_{0}} \int_{t_{0}}^{t} e^{\alpha_{0}\left(s-t_{0}\right)}\|x(s)\| d s .
\end{gathered}
$$

Aplicando a Desigualdade de Gronwall, obtemos

$$
\begin{gathered}
e^{\alpha_{0}\left(t-t_{0}\right)}\|x(t)\| \leqslant N_{\alpha_{0}}\left\|x\left(t_{0}\right)\right\| e^{q N_{\alpha_{0}}\left(t-t_{0}\right)}<\eta e^{q N_{\alpha_{0}}\left(t-t_{0}\right)} \\
\Longrightarrow\|x(t)\|<\eta e^{\left(q N_{\alpha_{0}}-\alpha_{0}\right)\left(t-t_{0}\right)}, \quad t_{0} \leqslant t<T .
\end{gathered}
$$

Desta forma, $T$ pode ser tomado arbitrariamente grande. De fato, se supormos que $\eta=\|x(T)\|$, teremos

$$
\eta=\|x(T)\|=\lim _{t \rightarrow T}\|x(t)\|<\eta e^{\left(q N_{\alpha_{0}}-\alpha_{0}\right)\left(T-t_{0}\right)}<\eta,
$$

o que é um absurdo. E ainda da desigualdade (3.17), temos $\|x(t)\| \rightarrow 0$ quando $t \rightarrow \infty$.

Suponhamos, agora, que o espectro $\sigma(A)$ não intersepte o eixo imaginário e que contenha pontos localizados no interior do semi-plano direito. Consideremos a seguinte decomposição do espectro $\sigma(A)=\sigma_{+}(A) \cup \sigma_{-}(A)$, onde $\sigma_{+}(A)\left(\sigma_{-}(A)\right)$ é constituído dos valores espectrais com parte real positiva (negativa). Sejam $P_{+}$e $P_{-}$ as projeções espectrais correspondentes à decomposição do espectro e $X=X_{+} \oplus X_{-}$, a correspondente decomposição direta de $X$ em subespaços $X_{+}$e $X_{-}$invariantes por $A$. Uma vez que $X_{+}$e $X_{-}$também são invariantes pelo operador $e^{A t}, 0 \leqslant t<\infty$, a solução dada por (3.7) da equação (3.6) permanece no subespaço contendo o vetor inicial $x_{0}$. Podemos introduzir uma norma em cada um destes subespaços usando a fórmula (3.10) e substituindo $A$ por $-A$ em $X_{+}$da seguinte forma

$$
\|x\|_{A}=\int_{0}^{\infty}\left\|e^{A t} x\right\| d t, \quad x \in X_{-} \quad \text { e } \quad\|x\|_{-A}=\int_{0}^{\infty}\left\|e^{-A t} x\right\| d t, \quad x \in X_{+} .
$$


Agora, introduzimos uma nova norma em $X$, tomando

$$
\|x\|_{A}=\left\|P_{+} x\right\|_{-A}+\left\|P_{-} x\right\|_{A}=\int_{0}^{\infty}\left\|e^{-A t} P_{+} x\right\| d t+\int_{0}^{\infty}\left\|e^{A t} P_{-} x\right\| d t .
$$

Uma vez que em cada subespaço $X_{+}$e $X_{-}$, as normas $\|x\|_{-A}$ e $\|x\|_{A}$ são, pelo Lema 3.1.4, topologicamente equivalentes à norma $\|x\|$, então a norma $\|x\|_{A}$ é topologicamente equivalente à norma $\left\|P_{+} x\right\|+\left\|P_{-} x\right\|$, que é equivalente à norma $\|x\|$. Logo, para (3.18) também podemos escrever $m\|x\| \leqslant\|x\|_{A} \leqslant M\|x\|$.

Com base em (3.18), definimos a norma indefinida

$$
\langle\langle x\rangle\rangle_{A}=\left\|P_{+} x\right\|_{-A}-\left\|P_{-} x\right\|_{A} .
$$

Os argumentos que usaremos a seguir são análogos aos que usamos no Teorema 3.1.5.

Seja $x(t)$ uma solução da equação (3.5) e seja $\left[t_{0}, T\right]$ um intervalo em que $\|x(t)\|<$ $\eta$. Tomando $y(t)=e^{-A t} x(t)$, como em (3.11), obtemos $y^{\prime}(t)=e^{-A t} F(t, x)$. Logo,

$$
\left\|P_{+} x(t)\right\|_{-A}=\int_{0}^{\infty}\left\|e^{-A(\tau-t)} P_{+} y(t)\right\| d \tau=\int_{-t}^{\infty}\left\|e^{-A \tau} P_{+} y(t)\right\| d \tau .
$$

Estimaremos a derivada à direita da função $\left\|P_{+} x(t)\right\|_{-A^{*}}$. Escrevemos

$$
\begin{aligned}
& \frac{\left\|P_{+} x(t+h)\right\|_{-A}-\left\|P_{+} x(t)\right\|_{-A}}{h}=\frac{\int_{-t-h}^{\infty}\left\|e^{-A \tau} P_{+} y(t+h)\right\| d \tau-\int_{-t}^{\infty}\left\|e^{-A \tau} P_{+} y(t)\right\| d \tau}{h} \\
& =\frac{1}{h} \int_{-t-h}^{-t}\left\|e^{-A \tau} P_{+} y(t+h)\right\| d \tau+\int_{-t}^{\infty} \frac{\left\|e^{-A \tau} P_{+} y(t+h)\right\|-\left\|e^{-A \tau} P_{+} y(t)\right\|}{h} d \tau .
\end{aligned}
$$

Chamando de $I_{1}(h)$ e $I_{2}(h)$, respectivamente, a primeira e a segunda parcela da igualdade, temos

$$
\lim _{h \rightarrow 0}\left|I_{1}(h)\right|=\left\|e^{A t} P_{+} y(t)\right\|=\left\|P_{+} x(t)\right\|
$$

$\mathrm{e}$

$$
\begin{aligned}
\left|I_{2}(h)\right| & \leqslant \int_{-t}^{\infty}\left\|e^{-A \tau} P_{+} \frac{y(t+h)-y(t)}{h}\right\| d \tau=\int_{0}^{\infty}\left\|e^{-A s} P_{+} e^{A t} \frac{y(t+h)-y(t)}{h}\right\| d s \\
& =\left\|e^{A t} P_{+} \frac{y(t+h)-y(t)}{h}\right\|_{-A} .
\end{aligned}
$$

Logo,

$$
\varlimsup_{h \rightarrow 0}\left|I_{2}(h)\right| \leqslant\left\|e^{A t} P_{+} y^{\prime}(t)\right\|_{-A}=\left\|P_{+} F(t, x(t))\right\|_{-A} .
$$


De (3.19) e (3.20), temos

$$
\begin{aligned}
\frac{d^{+}\left\|P_{+} x(t)\right\|_{-A}}{d t} & =\lim _{h \rightarrow 0} \frac{\left\|P_{+} x(t+h)\right\|_{-A}-\left\|P_{+} x(t)\right\|_{-A}}{h} \geqslant \lim _{h \rightarrow 0}\left|I_{1}(h)\right|-\varlimsup_{h \rightarrow 0}\left|I_{2}(h)\right| \\
& \geqslant\left\|P_{+} x(t)\right\|-\left\|P_{+} F(t, x(t))\right\|_{-A} \geqslant \frac{1}{M}\left\|P_{+} x(t)\right\|_{-A}-\left\|P_{+} F(t, x(t))\right\|_{-A} .
\end{aligned}
$$

Assim,

$$
\left\|P_{+} x(t)\right\|_{-A}-\left\|P_{+} x(\tau)\right\|_{-A} \geqslant \int_{\tau}^{t}\left[\frac{1}{M}\left\|P_{+} x(s)\right\|_{-A}-\left\|P_{+} F(s, x(s))\right\|_{-A}\right] d s .
$$

Analogamente, deduzimos a desigualdade

$$
\begin{aligned}
\frac{d^{+}\left\|P_{-} x(t)\right\|_{A}}{d t} & =\lim _{h \rightarrow 0} \frac{\left\|P_{-} x(t+h)\right\|_{A}-\left\|P_{-} x(t)\right\|_{A}}{h} \leqslant-\left\|P_{-} x(t)\right\|+\left\|P_{-} F(t, x(t))\right\|_{A} \\
& \leqslant-\frac{1}{M}\left\|P_{-} x(t)\right\|_{A}+\left\|P_{-} F(t, x(t))\right\|_{A},
\end{aligned}
$$

e a estimativa

$$
\left\|P_{-} x(t)\right\|_{A}-\left\|P_{-} x(\tau)\right\|_{A} \leqslant \int_{\tau}^{t}\left[-\frac{1}{M}\left\|P_{-} x(s)\right\|_{A}+\left\|P_{-} F(s, x(s))\right\|_{A}\right] d s .
$$

Subtraindo (3.22) de (3.21), temos

$$
\langle\langle x(t)\rangle\rangle_{A} \geqslant\langle\langle x(\tau)\rangle\rangle_{A}+\beta(t, \tau)
$$

onde $\beta(t, \tau)=\int_{\tau}^{t}\left[\frac{1}{M}\|x(s)\|_{A}-\|F(s, x(s))\|_{A}\right] d s$.

Suponhamos que $F$ satisfaça (3.4), com $q$ suficientemente pequeno $\left(q<q_{0}=\frac{m}{M}\right)$. Assim

$$
\frac{1}{M}\|F(t, x)\|_{A} \leqslant\|F(t, x)\| \leqslant q\|x\| \leqslant \frac{q}{m}\|x\|_{A} \Longrightarrow\|F(t, x)\|_{A} \leqslant \frac{q M}{m}\|x\|_{A} .
$$
Logo,

$$
\beta(t, \tau) \geqslant \int_{\tau}^{t}\left(\frac{1}{M}-\frac{q M}{m}\right)\|x(s)\|_{A} d s=\alpha \int_{\tau}^{t}\|x(s)\|_{A} d s
$$

onde $\alpha=\frac{1}{M}-\frac{q M}{m}>0$. De (3.23) e (3.24), obtemos

$$
\langle\langle x(t)\rangle\rangle_{A}-\langle\langle x(\tau)\rangle\rangle_{A} \geqslant \alpha \int_{\tau}^{t}\|x(s)\|_{A} d s, \quad q<q_{0}, \quad t_{0} \leqslant \tau \leqslant t<T .
$$


A desigualdade (3.25) significa que a norma indefinida $\langle\langle x(t)\rangle\rangle_{A}$ de uma solução $x(t)$ cresce com $t$ crescendo, quando a condição $\|x(t)\|<\eta$ é satisfeita. De fato, de (3.25) temos

$$
\frac{d}{d t}\langle\langle x(t)\rangle\rangle_{A} \geqslant \alpha\|x(t)\|_{A} \geqslant \alpha m\|x(t)\|>0 .
$$

Notemos também que a decomposição

$$
x(t)=e^{A t} x_{0}=P_{-} e^{A t} x_{0}+P_{+} e^{A t} x_{0}=e^{A t} P_{-} x_{0}+e^{A t} P_{+} x_{0}
$$

implica que qualquer solução, para a qual $P_{+} x_{0} \neq 0$, cresce ilimitadamente.

Como conseqüência, temos o seguinte resultado:

Teorema 3.1.6 Suponhamos que o espectro $\sigma(A)$ não intersepte o eixo imaginário $e$ o conjunto espectral $\sigma_{+}(A)$ seja não vazio. Suponhamos que exista um número $q_{0}$ dependendo somente do operador A tal que a função $F(t, x)$ satisfaça a condição (3.4) para $q<q_{0}$. Então a solução nula da equação (3.5) é instável para $t \rightarrow \infty$. E ainda, toda solução não nula $x(t)$ desta equação para a qual

$$
\left\langle\left\langle x\left(t_{0}\right)\right\rangle\right\rangle_{A} \geqslant 0
$$

sai de qualquer bola fechada $\|x\| \leqslant \eta_{1}$ de raio $\eta_{1}<\eta$ em algum $t>t_{0}$.

Demonstração: Uma vez que $\langle\langle x(t)\rangle\rangle_{A}$ é crescente, segue de (3.26) que a desigualdade estrita $\gamma=\left\langle\left\langle x\left(t_{0}^{\prime}\right)\right\rangle\right\rangle_{A}>0$ vale para $t_{0}^{\prime}>t_{0}$.

Como $\langle\langle x(t)\rangle\rangle_{A}=\left\|P_{+} x(t)\right\|_{-A}-\left\|P_{-} x(t)\right\|_{A} \leqslant\left\|P_{+} x(t)\right\|_{-A}+\left\|P_{-} x(t)\right\|_{A}=\|x(t)\|_{A}$, e usando (3.25), obtemos $\|x(t)\|_{A} \geqslant\langle\langle x(t)\rangle\rangle_{A} \geqslant \alpha \int_{t_{0}^{\prime}}^{t}\|x(s)\|_{A} d s+\gamma \Longrightarrow-\|x(t)\|_{A} \leqslant \alpha \int_{t_{0}^{\prime}}^{t}\left(-\|x(s)\|_{A}\right) d s-\gamma$.

Pela Desigualdade de Gronwall, temos

$$
-\|x(t)\|_{A} \leqslant-\gamma e^{\alpha\left(t-t_{0}^{\prime}\right)} \Longrightarrow\|x(t)\|_{A} \geqslant \gamma e^{\alpha\left(t-t_{0}^{\prime}\right)} \Longrightarrow M\|x(t)\| \geqslant\|x(t)\|_{A} \geqslant \gamma e^{\alpha\left(t-t_{0}^{\prime}\right)} .
$$

Logo,

$$
\|x(t)\| \geqslant \frac{\gamma}{M} e^{\alpha\left(t-t_{0}^{\prime}\right)}
$$

Tomando $t$ suficientemente grande em (3.27), temos $\|x(t)\| \geqslant \eta_{1}$ para $\eta_{1}<\eta$. 
Finalmente, se $P_{-} x\left(t_{0}\right)=0$, a condição (3.26) é automaticamente satisfeita sempre que $x\left(t_{0}\right) \neq 0$, pois $\left\langle\left\langle x\left(t_{0}\right)\right\rangle\right\rangle_{A}=\left\|P_{+} x\left(t_{0}\right)\right\|_{-A} \geqslant 0$.

Portanto, podemos encontrar uma solução de (3.5) com uma condição inicial $\left\|x\left(t_{0}\right)\right\|$ arbitrariamente pequena, satisfazendo a condição $\|x(t)\| \geqslant \eta_{1}$ para $t$ suficientemente grande. Isto implica a instabilidade da solução nula da equação (3.5).

Observação: Suponhamos que a condição $\|F(t, x)\| \leqslant q\|x\|$ seja satisfeita para $-\infty<t \leqslant t_{0}$ e o conjunto espectral $\sigma_{-}(A)$ seja não vazio. Então uma solução $x(t)$ satisfazendo a condição $\left\langle\left\langle x\left(t_{0}\right)\right\rangle\right\rangle_{A} \leqslant 0$ sai de qualquer bola fechada $\|x\| \leqslant \eta_{1}<\eta$, quando $t \rightarrow-\infty$.

Temos também outro tipo de condição sobre $F(t, x)$, dada por

$$
\|F(t, x)\| \leqslant q\|x\|^{1+p}, \quad p>0, \quad\|x\| \leqslant \eta \quad \text { e } \quad t \geqslant t_{0} .
$$

Notemos que a condição do Teorema 3.1.6 relativa a $F(t, x)$ é satisfeita, uma vez que a estimativa (3.28) em uma bola fechada suficientemente pequena implica a estimativa (3.4), com um $q$ suficientemente pequeno.

Deduziremos, com base em (3.28), um teorema de instabilidade que, de certa maneira, é mais geral que o Teorema 3.1.6, uma vez que a suposição que $\sigma(A)$ não intersepta o eixo imaginário é derrubada. Entretanto, omitiremos sua demonstração, que pode ser encontrada em [7], pág. 291, Teorema 2.3.

Teorema 3.1.7 Suponhamos que o espectro $\sigma(A)$ contenha pontos localizados no interior do semi-plano direito e que a condição (3.28) seja satisfeita. Então a solução nula da equação (3.5) é instável.

\subsubsection{Estabilidade dos Equilíbrios da Equação Autônoma}

Consideremos a equação não linear autônoma

$$
\frac{d x}{d t}=f(x), \quad t_{0} \leqslant t<\infty .
$$

Dizemos que $x_{0}$ é uma solução de equilíbrio (ponto crítico) de (3.29), se $f\left(x_{0}\right)=0$. Analogamente, como argumentamos anteriormente, se a função $f(x)$ for continuamente Fréchet diferenciável em uma vizinhança do ponto $x_{0}$, então, pela Fórmula de 
Taylor, $f(x)=f\left(x_{0}\right)+d f\left(x_{0}\right)\left(x-x_{0}\right)+F\left(x-x_{0}\right)$. Logo a diferença $y(t)=x(t)-x_{0}$ satisfaz a equação

$$
\frac{d y}{d t}=A y+F(y)
$$

onde $A=d f\left(x_{0}\right)$ e $F$ satisfaz $\|F(y)\| \leqslant q\|y\|$, para $\|y\| \leqslant \eta$ e $t \geqslant t_{0}$.

Com base no que vimos, podemos concluir que o comportamento da solução nula da equação não linear (3.30) é determinado pelo espectro do operador linear limitado $A=d f\left(x_{0}\right)$. Então, se o espectro $\sigma(A)$ está localizado no interior do semi-plano esquerdo, a solução nula da equação (3.30), e daí também a solução de equilíbrio $x=$ $x_{0}$ da equação (3.29), é uniformemente e assintoticamente estável (Teorema 3.1.5). Entretanto, se os pontos de $\sigma(A)$ também estão localizados no interior do semiplano direito, podemos afirmar que a solução nula de (3.30) é instável quando: $\sigma(A)$ não intersepta o eixo imaginário (Teorema 3.1.6) ou uma condição do tipo (3.28) é satisfeita (Teorema 3.1.7). A condição (3.28) é satisfeita, por exemplo, se a função $f(x)$ for duas vezes Fréchet diferenciável pois, neste caso, pela Fórmula de Taylor teremos $\|F(x)\| \leqslant q\|x\|^{2}$.

\subsection{Estabilidade por Primeira Aproximação e a Diferencial de Hadamard}

Nesta seção, veremos que estabilidade por primeira aproximação linear não vale se somente Hadamard diferenciabilidade (ver Definição 2.1.9, (ii)) for assumida. Daremos um contra-exemplo de uma equação autônoma não linear da forma (3.29), com a função $f$ sendo Hadamard diferenciável mas não Fréchet diferenciável, e veremos que, apesar dos pontos pertencentes ao espectro da linearização $A=d f\left(x_{0}\right)$, em torno de um determinado ponto equilíbrio $x_{0}$, possuírem parte real negativa, isto é, $\sigma(A)$ está localizado no semi-plano esquerdo, o ponto de equilíbrio $x_{0}$ não é localmente estável para a equação não linear (3.29). Os resultados que veremos nesta seção foram discutidos por Pereira em [34].

Consideremos a equação não linear autônoma

$$
\frac{\partial u(x, t)}{\partial t}=-u(x, t)+\tanh (\beta J * u(x, t)+h),
$$


onde $u(x, t)$ é uma função real sobre $\mathbb{R} \times \mathbb{R}_{+}, \beta>0, h$ é uma constante não negativa, $J \in C^{1}(\mathbb{R})$ é uma função par não negativa com integral igual a um e suporte compacto no intervalo $[-1,1]$, e $*$ denota o produto de convolução, dado por

$$
(J * u)(x)=\int_{\mathbb{R}} J(x-y) u(y) d y .
$$

Antes de analisarmos o problema da estabilidade das soluções de equilíbrio, por primeira aproximação linear, consideremos o problema de Cauchy constituído pela equação (3.31) e a condição inicial $u(x, 0)=u_{0}$. Veremos que este problema é bem posto em um espaço de Banach apropriado. Para tanto, seja $L^{p}(\mathbb{R}), 1 \leqslant p<\infty$, o espaço de Banach de todas as funções $u$ mensuráveis tais que a p-ésima potência do valor absoluto $|u|$ seja integrável sobre $\mathbb{R}$, com a norma $\|u\|_{L^{p(\mathbb{R})}}=\left(\int_{\mathbb{R}}|u(x)|^{p} d x\right)^{\frac{1}{p}}$. Consideremos agora $L_{l o c}^{1}(\mathbb{R})$ o espaço de todas as funções $u \in L^{1}(I)$, em qualquer subconjunto compacto $I$ em $\mathbb{R}$ e tomemos o espaço de Banach $L^{2}(\mathbb{R}, \rho)$, definido por

$$
L^{2}(\mathbb{R}, \rho)=\left\{u \in L_{\text {loc }}^{1}(\mathbb{R}): \quad \int_{\mathbb{R}} u^{2}(x) \rho(x) d x<\infty\right\}
$$

com a norma $\|u\|_{L^{2}(\mathbb{R}, \rho)}=\left(\int_{\mathbb{R}} u^{2}(x) \rho(x) d x\right)^{\frac{1}{2}}$, onde $\rho$ é uma função integrável ( $\rho$ é uma função "peso", que faz com que as funções constantes estejam incluídas no espaço $\left.L^{2}(\mathbb{R}, \rho)\right)$. Podemos tomar a função $\rho(x)=\frac{1}{\pi}\left(1+x^{2}\right)^{-1}$, cuja integral sobre $\mathbb{R}$ é igual a um. O espaço de Sobolev de ordem superior $H^{k}(\mathbb{R}, \rho)$ correspondente é o espaço das funções $u \in L^{2}(\mathbb{R}, \rho)$ cujas derivadas generalizadas (ver [37], pág. 232, Definição 21.2) até ordem $k$ também estão em $L^{2}(\mathbb{R}, \rho)$ com a norma

$$
\|u\|_{H^{k}(\mathbb{R}, \rho)}=\left(\sum_{i=0}^{k}\left\|\frac{\partial^{i} u}{\partial x^{i}}\right\|_{L^{2}(\mathbb{R}, \rho)}^{2}\right)^{\frac{1}{2}} .
$$

Não entraremos em detalhes sobre estes espaços, porém os mesmos podem ser encontrados por exemplo em [37] e [39].

Definindo

$$
F(u)=-u+\tanh (\beta J * u+h)
$$

mostraremos que $F$ é uma função em $L^{2}(\mathbb{R}, \rho)$ globalmente Lipschitziana, se a condição

$$
\sup \{\rho(x): y-1 \leqslant x \leqslant y+1\} \leqslant K \rho(y)
$$

for satisfeita para uma constante $K>0$ e para todo $y \in \mathbb{R}$. 
Inicialmente, verifiquemos que é possível encontrarmos $K>0$ satisfazendo a condição (3.33). Em vez de analisarmos a condição $\rho(y-1) \leqslant K \rho(y)$, para simplificar os cálculos, analisaremos a condição $\rho(y) \leqslant K \rho(y+1)$, que é equivalente a $\frac{\rho(y)}{\rho(y+1)} \leqslant K$. Mas

$$
\begin{gathered}
\frac{\rho(y)}{\rho(y+1)}=\frac{\frac{1}{\pi} \frac{1}{1+y^{2}}}{\frac{1}{\pi} \frac{1}{1+(y+1)^{2}}}=\frac{1+(y+1)^{2}}{1+y^{2}}=\frac{1+y^{2}+2 y+1}{1+y^{2}}=1+\frac{2 y}{1+y^{2}}+\frac{1}{1+y^{2}} . \\
\text { Como }\left|\frac{y}{1+y^{2}}\right| \leqslant\left|\frac{y^{2}}{1+y^{2}}\right|<1 \text { e }\left|\frac{1}{1+y^{2}}\right| \leqslant 1 \text {, então } \\
\frac{\rho(y)}{\rho(y+1)}<1+2+1=4 \Longrightarrow \rho(y)<4 \rho(y+1) .
\end{gathered}
$$

Portanto, podemos tomar $K=3$.

Também é possível encontrarmos $K$, arbitrariamente próxima de um, escolhendo $\rho$ adequadamente satisfazendo a condição (3.33). Com esta finalidade, definimos uma função $f$ sobre $\mathbb{R}$ como sendo $f(x) \equiv \frac{1}{\pi}\left(1+x^{2}\right)^{-1}$ e tomamos $f_{\epsilon}(x)=\epsilon f(\epsilon x)$, para todo $x \in \mathbb{R}$ e $\epsilon>0$ arbitrário. Assim

$$
\int_{\mathbb{R}} f_{\epsilon}(x) d x=\int_{\mathbb{R}} \epsilon f(\epsilon x) d x=\int_{\mathbb{R}} f(y) d y=\int_{\mathbb{R}} f(x) d x,
$$

onde na penúltima igualdade tomamos $y=\epsilon x$. Assim, $f_{\epsilon}$ e $f$ possuem a mesma integral. Agora, mostraremos que $f_{\epsilon}$ satisfaz a condição (3.33), e ainda, se $\epsilon$ é suficientemente pequeno, a constante $K$ fica arbitrariamente próxima de um. De fato, a condição $f_{\epsilon}(y) \leqslant K f_{\epsilon}(y+1)$ é equivalente a

$$
\frac{f_{\epsilon}(y)}{f_{\epsilon}(y+1)} \leqslant K \Longleftrightarrow \frac{\epsilon f(\epsilon y)}{\epsilon f(\epsilon y+\epsilon)} \leqslant K \Longleftrightarrow \frac{f(z)}{f(z+\epsilon)} \leqslant K, \quad \text { onde } z=\epsilon y .
$$

Mas, $\frac{f(z)}{f(z+\epsilon)}=1+\frac{2 \epsilon z+\epsilon^{2}}{1+z^{2}}$, e uma vez que $\left|\frac{z}{1+z^{2}}\right|<1$ e $\left|\frac{1}{1+z^{2}}\right| \leqslant 1$, temos

$$
\lim _{\epsilon \rightarrow 0} \frac{2 \epsilon z+\epsilon^{2}}{1+z^{2}}=\lim _{\epsilon \rightarrow 0} 2 \epsilon \frac{z}{1+z^{2}}+\lim _{\epsilon \rightarrow 0} \epsilon^{2} \frac{1}{1+z^{2}}=0 .
$$

Logo, $\frac{f(z)}{f(z+\epsilon)} \rightarrow 1$ e, conseqüentemente, $\frac{f_{\epsilon}(y)}{f_{\epsilon}(y+1)} \rightarrow 1$, quando $\epsilon$ é suficientemente pequeno. Portanto, a função $\rho(x)=f_{\epsilon}(x)$ satisfaz a condição (3.33), e ainda, a constante $K$ fica arbitrariamente próxima de um, para $\epsilon$ suficientemente pequeno.

Agora já podemos provar o seguinte resultado: 
Teorema 3.2.1 Suponhamos que a função $\rho(x)$ satisfaça (3.33), para uma constante $K$ positiva e todo $y \in \mathbb{R}$. Então, a função $F$ é globalmente Lipschitziana em $L^{2}(\mathbb{R}, \rho)$, com

$$
\|F(u)-F(v)\|_{L^{2}(\mathbb{R}, \rho)} \leqslant(1+\beta \sqrt{K})\|u-v\|_{L^{2}(\mathbb{R}, \rho)} .
$$

Demonstração: Como $J$ é limitada com suporte compacto no intervalo $[-1,1]$, então $(J * u)(x)$ está bem definido para $u \in L_{\text {loc }}^{1}(\mathbb{R})$, pois

$$
\left|\int_{\mathbb{R}} J(x-y) u(y) d y\right|=\left|\int_{-1}^{1} J(x-y) u(y) d y\right| \leqslant \int_{-1}^{1}|J(x-y)||u(y)| d y<\infty .
$$

Uma vez que $|\tanh (\beta * u+h)(x)|<1$ e $\rho$ é integrável, então para $u \in L^{2}(\mathbb{R}, \rho)$ temos $F(u) \in L_{\text {loc }}^{1}(\mathbb{R})$ e ainda,

$$
\begin{aligned}
& \int_{\mathbb{R}} F(u)^{2} \rho(x) d x=\int_{\mathbb{R}}[-u(x)+\tanh (\beta J * u+h)(x)]^{2} \rho(x) d x \\
& =\int_{\mathbb{R}} u^{2}(x) \rho(x) d x+2 \int_{\mathbb{R}} u(x)[-\tanh (\beta J * u+h)(x)] \rho(x) d x \\
& +\int_{\mathbb{R}} \tanh ^{2}(\beta J * u+h)(x) \rho(x) d x<\int_{\mathbb{R}} u^{2}(x) \rho(x) d x+2 \int_{\mathbb{R}} u(x) \rho(x) d x+\int_{\mathbb{R}} \rho(x) d x<\infty .
\end{aligned}
$$

Daí, $F(u) \in L^{2}(\mathbb{R}, \rho)$ se $u \in L^{2}(\mathbb{R}, \rho)$. E ainda

$$
\begin{aligned}
\|J * u\|_{L^{2}(\mathbb{R}, \rho)}^{2} & =\int_{\mathbb{R}}|(J * u)(x)|^{2} \rho(x) d x \leqslant \int_{\mathbb{R}}\left(\int_{\mathbb{R}}|J(x-y)||u(y)| d y\right)^{2} \rho(x) d x \\
& =\int_{\mathbb{R}}\left(\int_{\mathbb{R}} J(x-y)^{\frac{1}{2}} J(x-y)^{\frac{1}{2}}|u(y)| d y\right)^{2} \rho(x) d x .
\end{aligned}
$$

Pela Desigualdade de Schwarz (ver [31], pág. 548), temos

$$
\begin{aligned}
& \|J * u\|_{L^{2}(\mathbb{R}, \rho)}^{2} \leqslant \int_{\mathbb{R}}\left[\left(\int_{\mathbb{R}}\left(J(x-y)^{\frac{1}{2}}\right)^{2} d y\right)^{\frac{1}{2}}\left(\int_{\mathbb{R}}\left(J(x-y)^{\frac{1}{2}}\right)^{2}|u(y)|^{2} d y\right)^{\frac{1}{2}}\right]^{2} \rho(x) d x \\
& =\int_{\mathbb{R}}\left(\int_{\mathbb{R}} J(x-y) d y\right)\left(\int_{\mathbb{R}} J(x-y)|u(y)|^{2} d y\right) \rho(x) d x \\
& =\|J\|_{L^{1}(\mathbb{R})} \int_{\mathbb{R}}\left(\int_{\mathbb{R}} J(x-y)|u(y)|^{2} d y\right) \rho(x) d x \leqslant \int_{\mathbb{R}}\left(\int_{\mathbb{R}} J(x-y) \rho(x) d x\right)|u(y)|^{2} d y \\
& \leqslant \int_{\mathbb{R}}\left(\int_{\mathbb{R}} J(x-y) \sup \{\rho(x)\} d x\right)|u(y)|^{2} d y \leqslant \int_{\mathbb{R}}\left(\int_{\mathbb{R}} J(x-y) K \rho(y) d x\right)|u(y)|^{2} d y
\end{aligned}
$$




$$
\begin{gathered}
=\int_{\mathbb{R}}\left(\int_{\mathbb{R}} J(x-y) d x\right) K \rho(y)|u(y)|^{2} d y=\int_{\mathbb{R}} K \rho(y)|u(y)|^{2} d y=K\|u\|_{L^{2}(\mathbb{R}, \rho)}^{2} \\
\Longrightarrow\|J * u\|_{L^{2}(\mathbb{R}, \rho)} \leqslant \sqrt{K}\|u\|_{L^{2}(\mathbb{R}, \rho)} .
\end{gathered}
$$

Uma vez que $|\operatorname{sech}(\beta J * w+h)| \leqslant 1$, para qualquer $w$ pertencente ao segmento aberto $] v, u[$, então, pela Desigualdade do Valor Médio e por (3.34), temos

$$
\begin{aligned}
& \|F(u)-F(v)\|_{L^{2}(\mathbb{R}, \rho)} \leqslant\|u-v\|_{L^{2}(\mathbb{R}, \rho)}+\|\tanh (\beta J * u+h)-\tanh (\beta J * v+h)\|_{L^{2}(\mathbb{R}, \rho)} \\
& \leqslant\|u-v\|_{L^{2}(\mathbb{R}, \rho)}+\left|\operatorname{sech}^{2}(\beta J * w+h)\right|\|(\beta J * u+h)-(\beta J * v+h)\|_{L^{2}(\mathbb{R}, \rho)} \\
& \leqslant\|u-v\|_{L^{2}(\mathbb{R}, \rho)}+\|\beta J *(u-v)\|_{L^{2}(\mathbb{R}, \rho)} \leqslant\|u-v\|_{L^{2}(\mathbb{R}, \rho)}+\beta \sqrt{K}\|u-v\|_{L^{2}(\mathbb{R}, \rho)} \\
& \Longrightarrow\|F(u)-F(v)\|_{L^{2}(\mathbb{R}, \rho)} \leqslant(1+\beta \sqrt{K})\|u-v\|_{L^{2}(\mathbb{R}, \rho)} \cdot \quad \square
\end{aligned}
$$

Como $F$ é uma função globalmente Lipschitziana em $L^{2}(\mathbb{R}, \rho)$, segue do Corolário 2.5.2 que para qualquer condição inicial $u(x, 0)=u_{0} \in L^{2}(\mathbb{R}, \rho)$, a equação (3.31) tem uma única solução globalmente definida em $C\left(\left[0, \infty\left[, L^{2}(\mathbb{R}, \rho)\right) \cap C^{1}(] 0, \infty\left[, L^{2}(\mathbb{R}, \rho)\right)\right.\right.$, que é contínua em relação à condição inicial $u_{0}$. Então, denotaremos por $T(t)$ o semifluxo global gerado por (3.31) em $L^{2}(\mathbb{R}, \rho)$.

Analisando agora as soluções de equilíbrio da equação (3.31), segue que estas satisfazem a equação $u=\tanh (\beta J * u+h)$. Uma vez que o gráfico da função $\tanh (\beta J * u+h)$ é limitado inferiormente por -1 e superiormente por 1 , podemos afirmar que todos os pontos de equilíbrio possuem imagem limitada. Se $0<\beta \leqslant$ 1, então a equação (3.31) possui um único ponto de equilíbrio e, no caso em que $h=0$, este ponto é exatamente a origem. Se $\beta>1$ e $0 \leqslant h<h_{*}$, onde $h_{*}$ é implicitamente definido pela equação (3.35) a seguir, então (3.31) possui três pontos de equilíbrio espacialmente homogêneos, que denotaremos por $m_{\beta}^{0}, m_{\beta}^{+}$e $m_{\beta}^{-}$, cada um deles identicamente igual a uma das três soluções da equação

$$
m_{\beta}=\tanh \left(\beta m_{\beta}+h\right)
$$

onde $J * m_{\beta}=\int_{\mathbb{R}} J(x-y) m_{\beta} d y=m_{\beta} \int_{\mathbb{R}} J(x-y) d y=m_{\beta}$. Convencionaremos que $m_{\beta}^{-}(h)<m_{\beta}^{0}(h) \leqslant 0<m_{\beta}^{+}(h)$ e $\left|m_{\beta}^{-}(h)\right|<m_{\beta}^{+}(h)$.

Consideraremos agora o caso em que $\beta>1$ e $0<h<h_{*}$. Mostraremos que a função $F$ em $L^{2}(\mathbb{R}, \rho)$, dada por (3.32), é Hadamard diferenciável mas não é Fréchet 
diferenciável, e provaremos que o espectro da linearização, com a diferencial de Hadamard, em torno do ponto de equilíbrio $m_{\beta}^{-}$está localizado sobre o semi-plano $\Re(\lambda)<-\gamma<0$, com $\gamma>0$, mas $m_{\beta}^{-}$não é localmente estável. Daí concluiremos que estabilidade por primeira aproximação linear não vale se somente Hadamard diferenciabilidade for assumida.

Começaremos com a definição de uma solução onda viajante para a equação (3.31).

Definição 3.2.2 Para qualquer $a \in \mathbb{R}$ denotamos por $S_{a}$ o operador sobre $C(\mathbb{R})$ definido para $m \in C(\mathbb{R})$ como $\left(S_{a} m\right)(x)=m(x-a)$. Dado $h>0$, dizemos que $\tilde{m}$ é uma solução onda viajante com velocidade $-\omega, \omega \in \mathbb{R}$, se $\tilde{m} \in C^{1}(\mathbb{R}), m_{t}:=S_{-\omega t} \tilde{m}$ satisfaz a equação (3.31), e as condições assintóticas

$$
\lim _{x \rightarrow \pm \infty} \tilde{m}(x)=m_{\beta}^{ \pm}(h) .
$$

Portanto, a onda viajante $\tilde{m}$ satisfaz a equação

$$
\omega \frac{\partial u}{\partial x}=-u+\tanh (\beta J * u+h) .
$$

Como convencionamos, $m_{\beta}^{+}$está à direita da origem, e uma vez que $h>0$, então a velocidade $-\omega$ da frente de onda é negativa e $\omega$ é positiva.

Em [9], De Masi, Gobron e Presutti mostraram que existe uma solução de (3.31) onda viajante conectanto $m_{\beta}^{-}$e $m_{\beta}^{+}$, logo $m_{\beta}^{-}$não é um ponto de equilíbrio estável para a equação (3.31). Aqui não entraremos em detalhes sobre este resultado, apenas enunciaremos o teorema que garante a existência e unicidade da onda viajante, a demonstração do mesmo pode ser encontrada em [9], Teorema 2.3.

Teorema 3.2.3 Existe um $h_{0}>0$ tal que para qualquer $\left.\left.h \in\right] 0, h_{0}\right]$, existe um $\omega(h)>$ 0 e uma onda viajante $\tilde{m}=\tilde{m}(\cdot ; h)$ com velocidade $-\omega(h)$. E ainda para qualquer onda $m \in C^{1}(\mathbb{R})$ tal que

$$
\lim _{x \rightarrow+\infty} \inf m(x)>m_{\beta}^{0}(h) \quad \text { e } \quad \lim _{x \rightarrow-\infty} \sup m(x)<m_{\beta}^{0}(h)
$$

existe uma constante $a \in \mathbb{R}$ tal que $m=S_{a} \tilde{m}$.

Teorema 3.2.4 A função F dada em (3.32) é Hadamard diferenciável, mas não é Fréchet diferenciável. 
Demonstração: Encontremos, inicialmente, a diferencial de Gâteaux $\delta F(u ; \cdot)$. Pela Definição 2.1.9, $(i)$, a diferencial de Gâteaux $\delta F(u ; v)$, com $v \in L^{2}(\mathbb{R}, \rho)$, é dada por

$$
\lim _{t \rightarrow 0} \frac{F(u+t v)-F(u)}{t}=\lim _{t \rightarrow 0} \frac{-t v+\tanh (\beta J *(u+t v)+h)-\tanh (\beta J * u+h)}{t} .
$$

Aplicando L'Hospital e usando o fato que $\operatorname{sech}^{2}(\beta J *(u+t v)+h)$ é uma função contínua, temos

$$
\begin{aligned}
\lim _{t \rightarrow 0} \frac{F(u+t v)-F(u)}{t} & =\lim _{t \rightarrow 0}\left[-v+\operatorname{sech}^{2}(\beta J *(u+t v)+h)(\beta J * v)\right] \\
& =-v+\operatorname{sech}^{2}(\beta J * u+h)(\beta J * v) .
\end{aligned}
$$

Assim, uma candidata a diferencial de Hadamard de $F$ é dada por

$$
\delta F(u ; v)=-v+\operatorname{sech}^{2}(\beta J * u+h)(\beta J * v), \quad \text { para todo } v \in L^{2}(\mathbb{R}, \rho) .
$$

Inicialmente, temos que $\delta F(u ; \cdot)$ é um operador linear em $L^{2}(\mathbb{R}, \rho)$. De fato, para todo $v, w \in L^{2}(\mathbb{R}, \rho)$ e escalar $\alpha$, temos

$$
\begin{aligned}
& \delta F(u ; v+\alpha w)=-(v+\alpha w)+\operatorname{sech}^{2}(\beta J * u+h)(\beta J *(v+\alpha w)) \\
& =-v+\operatorname{sech}^{2}(\beta J * u+h)(\beta J * v)-\alpha w+\operatorname{sech}^{2}(\beta J * u+h)(\alpha \beta J * w)=\delta F(u ; v)+\alpha \delta F(u ; w) .
\end{aligned}
$$

E ainda, $\delta F(u ; \cdot)$ é um operador linear limitado em $L^{2}(\mathbb{R}, \rho)$, pois de (3.34) temos

$$
\begin{aligned}
\|\delta F(u ; v)\|_{L^{2}(\mathbb{R}, \rho)} & \leqslant\|v\|_{L^{2}(\mathbb{R}, \rho)}+\left|\operatorname{sech}^{2}(\beta J * u+h)\right|\|\beta J * v\|_{L^{2}(\mathbb{R}, \rho)} \\
& \leqslant\|v\|_{L^{2}(\mathbb{R}, \rho)}+\beta \sqrt{K}\|v\|_{L^{2}(\mathbb{R}, \rho)}=(1+\beta \sqrt{K})\|v\|_{L^{2}(\mathbb{R}, \rho)} .
\end{aligned}
$$

Uma vez que $F$ tem diferencial de Gâteaux linear em $v$ e $F$ é globalmente Lipschitziana em $u$, então por (iv) da Proposição 2.1 .11 já poderíamos concluir que $F$ é Hadamard diferenciável em $u$, com a diferencial de Hadamard $D F(u) v=\delta F(u ; v)$. No entanto, mostraremos a seguir que isto realmente é verdade. Usando o Teorema 3.2 .1 e a Gâteaux diferenciabilidade de $F$, temos

$$
\begin{aligned}
& \left\|\frac{F(u+t v+t k)-F(u)-t \delta F(u ; v)}{t}\right\|_{L^{2}(\mathbb{R}, \rho)} \\
= & \left\|\frac{F(u+t v+t k)-F(u+t v)+F(u+t v)-F(u)-t \delta F(u ; v)}{t}\right\|_{L^{2}(\mathbb{R}, \rho)}
\end{aligned}
$$




$$
\begin{aligned}
& \leqslant\left\|\frac{F(u+t v+t k)-F(u+t v)}{t}\right\|_{L^{2}(\mathbb{R}, \rho)}+\left\|\frac{F(u+t v)-F(u)-t \delta F(u ; v)}{t}\right\|_{L^{2}(\mathbb{R}, \rho)} \\
& \leqslant(1+\beta \sqrt{K})\|k\|_{L^{2}(\mathbb{R}, \rho)}+\left\|\frac{F(u+t v)-F(u)}{t}-\delta F(u ; v)\right\|_{L^{2}(\mathbb{R}, \rho)} \rightarrow 0,
\end{aligned}
$$

quando $(t, k) \rightarrow(0,0)$. Portanto, pela Definição 2.1.9, $(i i), F$ é Hadamard diferenciável, com diferencial $D F(u) v=\delta F(u ; v)$.

Entretanto, $F$ não é Fréchet diferenciável. De fato, o Teorema 3.2.3 afirma que existe uma solução de (3.31) onda viajante conectanto $m_{\beta}^{-}$e $m_{\beta}^{+}$, o que nos assegura que $m_{\beta}^{-}$não é um ponto de equilíbrio localmente estável. Logo, pelo que vimos na Seção 3.1, podemos concluir que a função $F$ não é Fréchet diferenciável, pois se $F$ fosse Fréchet diferenciável, o ponto de equilíbrio $m_{\beta}^{-}$seria estável.

Uma vez que $F$ é Hadamard diferenciável, com a diferencial de Hadamard $D F(u)$ contínua em $u$, então, pela Fórmula de Taylor em torno do ponto de equilíbrio $m_{\beta}^{-}$,

$$
F\left(m_{\beta}^{-}+v\right)=D F\left(m_{\beta}^{-}\right) v+r\left(m_{\beta}^{-}, v\right), \quad \text { onde } \quad \lim _{v \rightarrow 0} \frac{r\left(m_{\beta}^{-}, v\right)}{\|v\|_{L^{2}(\mathbb{R}, \rho)}}=0,
$$

onde $D F\left(m_{\beta}^{-}\right) v=-v+\operatorname{sech}^{2}\left(\beta J * m_{\beta}^{-}+h\right)(\beta J * v)$ é a diferencial de Hadamard calculada em $m_{\beta}^{-}$. Como já vimos anteriormente $J * m_{\beta}^{-}=m_{\beta}^{-}$, e $m_{\beta}^{-}$satisfaz $m_{\beta}^{-}=$ $\tanh \left(\beta m_{\beta}^{-}+h\right)$. Uma vez que $1-\tanh ^{2}\left(\beta m_{\beta}^{-}+h\right)=\operatorname{sech}^{2}\left(\beta m_{\bar{\beta}}^{-}+h\right)$, então

$D F\left(m_{\beta}^{-}\right) v=-v+\beta\left[1-\left(m_{\beta}^{-}\right)^{2}\right] J * v \quad$ ou $\quad D F\left(m_{\beta}^{-}\right)(\cdot)=-I+\beta\left[1-\left(m_{\beta}^{-}\right)^{2}\right] J *(\cdot)$.

Considerando agora a equação linear associada a (3.31), dada por $v_{t}=D F\left(m_{\beta}^{-}\right) v$, temos o seguinte resultado:

Teorema 3.2.5 O espectro do operador $D F\left(m_{\beta}^{-}\right)$está localizado sobre o semi-plano $\Re(\lambda)<-\gamma<0, \operatorname{com} \gamma>0$.

Demonstração: Mostraremos que se $\Re(\lambda) \geqslant 0$, então $\left(\lambda I-D F\left(m_{\beta}^{-}\right)\right)$é um operador inversível e com inversa contínua. Desta forma, teremos $\lambda$ pertencente ao resolvente $\rho\left(D F\left(m_{\beta}^{-}\right)\right)$de $D F\left(m_{\beta}^{-}\right)$(ver Definição A.2.1). Temos,

$$
\begin{gathered}
\left(\lambda I-D F\left(m_{\beta}^{-}\right)\right)=\lambda I+I-\beta\left[1-\left(m_{\beta}^{-}\right)^{2}\right] J *(\cdot)=(1+\lambda) I-\beta\left[1-\left(m_{\beta}^{-}\right)^{2}\right] J *(\cdot) \\
\Longrightarrow \frac{1}{1+\lambda}\left(\lambda I-D F\left(m_{\beta}^{-}\right)\right)=I-\frac{\beta\left[1-\left(m_{\beta}^{-}\right)^{2}\right]}{1+\lambda} J *(\cdot) .
\end{gathered}
$$


Analisaremos o operador $A=\frac{c}{1+\lambda} J *(\cdot)$, onde $c=\beta\left[1-\left(m_{\beta}^{-}\right)^{2}\right]$. De (3.34) e do fato que $K$ pode ser tomada arbitrariamente próxima de 1 , temos $\|J *(\cdot)\|<1$.

Mostremos agora que $\left|\frac{c}{1+\lambda}\right|<1$. Uma vez que $\Re(\lambda) \geqslant 0$, então $|1+\lambda| \geqslant 1$. Logo, basta mostrarmos que $c<1$. Com esta finalidade, seja $g(x)=\tanh (\beta x+h)$, com $\left.x \in]-\infty, m_{\beta}^{0}\right]$, então $g^{\prime}(x)=\beta \operatorname{sech}^{2}(\beta x+h)$. Logo

$$
g^{\prime}\left(m_{\beta}^{-}\right)=\beta \operatorname{sech}^{2}\left(\beta m_{\beta}^{-}+h\right)=\beta\left[1-\tanh ^{2}\left(\beta m_{\beta}^{-}+h\right)\right]=\beta\left[1-\left(m_{\beta}^{-}\right)^{2}\right]=c .
$$

Daí, basta verificarmos que $g^{\prime}\left(m_{\beta}^{-}\right)<1$. Para tanto, trabalharemos com o conceito de função convexa (ver [28], pág. 226). Mostremos que $\left.\left.g^{\prime \prime}(x)>0 \mathrm{em}\right]-\infty, m_{\beta}^{0}\right]$.

$$
\begin{aligned}
g^{\prime \prime}(x) & =2 \beta \operatorname{sech}(\beta x+h)[-\beta \tanh (\beta x+h) \operatorname{sech}(\beta x+h)] \\
& \left.\left.=-2 \beta^{2} \operatorname{sech}^{2}(\beta x+h) \tanh (\beta x+h) \geqslant 0, \quad \text { para todo } x \in\right]-\infty, m_{\beta}^{0}\right] .
\end{aligned}
$$

Notemos que a única possibilidade de $g^{\prime \prime}(x)=0$ é em $x=m_{\beta}^{0}=0$, e isto ocorre quando $h=0$, mas estamos excluindo este caso. Logo, $\left.\left.g^{\prime \prime}(x)>0 \mathrm{em}\right]-\infty, m_{\beta}^{0}\right]$.

Assim, por [28], pág. 226, Teorema 11, segue que $g$ é convexa em ] $\left.-\infty, m_{\beta}^{0}\right]$. Em particular, é convexa em $\left[-m_{\beta}^{+}, m_{\beta}^{-}\right]$. Daí, por definição, qualquer ponto $(x, g(x))$ do gráfico de $g$ está situado abaixo da reta secante que liga os pontos $\left(-m_{\beta}^{+}, g\left(-m_{\beta}^{+}\right)\right)$e $\left(m_{\beta}^{-}, g\left(m_{\beta}^{-}\right)\right)$. Logo

$$
g(x) \leqslant g\left(m_{\beta}^{-}\right)+g^{\prime}\left(m_{\beta}^{-}\right)\left(x-m_{\beta}^{-}\right), \quad \text { para todo } x \in\left[-m_{\beta}^{+}, m_{\beta}^{-}\right] .
$$

Agora, suponhamos, por contradição, que $g^{\prime}\left(m_{\beta}^{-}\right) \geqslant 1$. Temos, então, dois casos a considerar:

$\left.1^{\circ}\right)$ Se $g^{\prime}\left(m_{\beta}^{-}\right)=1$, segue de (3.36) que $g(x) \leqslant m_{\beta}^{-}+x-m_{\beta}^{-}=x$. O que é um absurdo, uma vez que $x<g(x)$ para todo $x \in\left[-m_{\beta}^{+}, m_{\beta}^{-}\right]$.

$\left.2^{\circ}\right)$ Se $g^{\prime}\left(m_{\beta}^{-}\right)>1$, uma vez que $g^{\prime}$ é uma função contínua em $\left[-m_{\beta}^{+}, m_{\beta}^{-}\right]$, então existe $\delta>0$ tal que $g^{\prime}(x)>1$ para todo $\left.\left.x \in\right] m_{\beta}^{-}-\delta, m_{\beta}^{-}\right]$. Daí, $x-m_{\beta}^{-} \leqslant 0$ e por (3.36) obtemos

$$
g^{\prime}\left(m_{\beta}^{-}\right)\left(x-m_{\beta}^{-}\right) \leqslant x-m_{\beta}^{-} \Longrightarrow g(x) \leqslant m_{\beta}^{-}+x-m_{\beta}^{-}=x .
$$

Novamente um absurdo, pois $x<g(x)$ em $\left.] m_{\beta}^{-}-\delta, m_{\beta}^{-}\right]$. Assim, só podemos ter $g^{\prime}\left(m_{\beta}^{-}\right)<1$.

Portanto, $\|A\|<1$. Daí, pelo Teorema A.2.2, $(I-A)^{-1}$ existe e é um operador limitado. Logo, $\frac{1}{1+\lambda}\left(\lambda I-D F\left(m_{\beta}^{-}\right)\right)$é um operador inversível, com inversa 
limitada. Conseqüentemente, $\left(\lambda I-D F\left(m_{\beta}^{-}\right)\right)^{-1}$ existe e é limitado para $\Re(\lambda) \geqslant 0$. Daí, o espectro do operador $\sigma\left(D F\left(m_{\beta}^{-}\right)\right)$só pode estar localizado sobre o semi-plano $\Re(\lambda)<-\gamma<0$.

Do Teorema 3.2.5 e supondo que podemos utilizar os resultados da Subseção 3.1.1, podemos concluir que o ponto de equilíbrio $m_{\beta}^{-}$é estável. Entretanto, já vimos que isto não é verdade. Portanto, a estabilidade por primeira aproximação linear não vale se somente Hadamard diferenciabilidade for assumida.

Mesmo diante do que acabamos de ver, ainda é possível encontrarmos um conjunto apropriado, em que a solução de equilíbrio $m_{\beta}^{-}$é localmente estável. Mais especificamente, mostraremos que $m_{\beta}^{-}$é localmente estável para o fluxo em $L^{2}(\mathbb{R}, \rho) \cap \Sigma^{0}$, onde $\Sigma^{0}$ é o conjunto invariante

$$
\begin{aligned}
& \left.\Sigma^{0}:=\left\{u \in C_{b}(\mathbb{R}): \quad\|u\|_{\infty} \leqslant 1, u \text { é par, crescente em }\right]-\infty, 0\right] \mathrm{e} \\
& \left.u(x)-m_{\beta}^{-} \in L^{2}(\mathbb{R})\right\}
\end{aligned}
$$

onde $C_{b}(\mathbb{R})$ é o espaço de Banach das funções contínuas e limitadas, com a norma do supremo denotada por $\|\cdot\|_{\infty}$.

Mas antes precisamos do lema a seguir, cuja demonstração pode ser encontrada em [10], pág. 702, Teorema 2.7.

Lema 3.2.6 Se u e v são soluções de (3.31) em $C_{b}(\mathbb{R})$ com $u(x, 0) \leqslant v(x, 0)$ para qualquer $x \in \mathbb{R}$, então $u(x, t) \leqslant v(x, t)$ para qualquer $x \in \mathbb{R}$ e $t \geqslant 0$.

Teorema 3.2.7 Dado $\epsilon>0$, temos que existe $\delta>0$ e $\tau>0$ tais que, se $u \in \Sigma^{0} e$ $\left\|u-m_{\beta}^{-}\right\|_{L^{2}(\mathbb{R}, \rho)}<\delta$, então $\left\|T(\tau) u-m_{\beta}^{-}\right\|_{\infty} \leqslant \epsilon$.

Demonstração: Seja $\epsilon>0$ e fixemos $\tau$ tal que $e^{-\tau} \leqslant \frac{\epsilon}{4}$. E ainda, para qualquer $\eta>0$ e $M>0$ existe $\delta_{1}>0$ tal que, se $u \in \Sigma^{0}$ e $\left\|u-m_{\beta}^{-}\right\|_{L^{2}(\mathbb{R}, \rho)}<\delta_{1}$, então $\left|u(x)-m_{\beta}^{-}\right| \leqslant \frac{\epsilon}{M}$ sempre que $|x| \geqslant \eta$. Uma vez que o fluxo $T(t)$ gerado por (3.31) é contínuo em $L^{2}(\mathbb{R}, \rho)$, existe $\delta_{2}$ tal que $\left\|T(s) u-m_{\beta}^{-}\right\|_{L^{2}(\mathbb{R}, \rho)} \leqslant \delta$ se $\left\|u-m_{\beta}^{-}\right\|_{L^{2}(\mathbb{R}, \rho)} \leqslant$ $\delta_{2}$, para $0 \leqslant s \leqslant \tau$. Tomamos $\eta$ e $M$ tais que $\beta\left(2 \gamma+\frac{\epsilon}{M}\right) \leqslant \frac{\epsilon}{2}$, onde $\gamma=\int_{-\eta}^{\eta} J(y) d y$ (é possível obtermos a estimativa uma vez que $\eta$ é pequeno), e seja $u_{0} \in \Sigma^{0}$ com $\left\|u_{0}-m_{\beta}^{-}\right\|_{L^{2}(\mathbb{R}, \rho)}<\delta=\delta_{2}$. Pela Fórmula da Variação das Constantes, a solução 
$u(x, t)$ de (3.31), satisfazendo a condição inicial $u_{0}(x)=u(x, 0)$, é dada por

$$
u(x, t)=e^{-t} u_{0}(x)+\int_{0}^{t} e^{s-t} \tanh (\beta(J * u)(x, s)+h) d s .
$$

Denotando agora por $u(\cdot, t)=(T(t) u)(\cdot)$ a solução de $(3.31)$ com condição inicial $u_{0}(\cdot)$, temos

$$
\begin{aligned}
& \left\|T(\tau) u_{0}-m_{\beta}^{-}\right\|_{\infty}=\left|u(0, \tau)-m_{\beta}^{-}\right| \leqslant\left|e^{-\tau}\left(u_{0}(0)-m_{\beta}^{-}\right)\right| \\
& +\int_{0}^{\tau} e^{-(\tau-s)}\left|\tanh (\beta J * u+h)(0, s)-\tanh \left(\beta J * m_{\beta}^{-}+h\right)\right| d s \\
& \leqslant \frac{\epsilon}{4}\left(\left|u_{0}(0)\right|+\left|m_{\beta}^{-}\right|\right)+\int_{0}^{\tau} e^{-(\tau-s)} \beta\left|J * u(0, s)-J * m_{\beta}^{-}\right| d s \\
& \leqslant \frac{\epsilon}{4}(1+1)+\int_{0}^{\tau} e^{-(\tau-s)} \beta\left(\int_{\mathbb{R}} J(y)\left|u(y, s)-m_{\beta}^{-}\right| d y\right) d s \\
& \leqslant \frac{\epsilon}{2}+\int_{0}^{\tau} e^{-(\tau-s)} \beta\left(\int_{-\eta}^{\eta} J(y)\left|u(y, s)-m_{\beta}^{-}\right| d y\right. \\
& \left.+\int_{-\infty}^{-\eta} J(y)\left|u(y, s)-m_{\beta}^{-}\right| d y+\int_{\eta}^{+\infty} J(y)\left|u(y, s)-m_{\beta}^{-}\right| d y\right) d s \\
& \leqslant \frac{\epsilon}{2}+\int_{0}^{\tau} e^{-(\tau-s)} \beta\left(2 \int_{-\eta}^{\eta} J(y) d y+\frac{\epsilon}{M} \int_{-\infty}^{-\eta} J(y) d y+\frac{\epsilon}{M} \int_{\eta}^{+\infty} J(y) d y\right) d s \\
& \leqslant \frac{\epsilon}{2}+\int_{0}^{\tau} e^{-(\tau-s)} \beta\left(2 \gamma+\frac{\epsilon}{M} \int_{-\infty}^{+\infty} J(y) d y\right) d s \\
& \leqslant \frac{\epsilon}{2}+\beta\left(2 \gamma+\frac{\epsilon}{M}\right) \int_{0}^{\tau} e^{-(\tau-s)} d s \leqslant \frac{\epsilon}{2}+\frac{\epsilon}{2} \int_{0}^{\tau} e^{-(\tau-s)} d s \leqslant \epsilon . \quad \square
\end{aligned}
$$

Corolário 3.2.8 O ponto de equilíbrio $m_{\beta}^{-}$é localmente estável para o fluxo em $L^{2}(\mathbb{R}, \rho) \cap \Sigma^{0}$.

Demonstração: Do Lema 3.2.6, segue que o ponto de equilíbrio $m_{\beta}^{-}$é localmente estável para o fluxo definido em $C_{b}(\mathbb{R})$. E o resultado segue do Teorema 3.2.7.

Observemos que o Corolário 3.2.8 garante estabilidade do ponto de equilíbrio $m_{\beta}^{-}$, sem utilizar os resultados de estabilidade por primeira aproximação linear da seção anterior, uma vez que já sabemos que isto não é possível, porque a função $F$ é apenas Hadamard diferenciável em $L^{2}(\mathbb{R}, \rho)$. Contudo, não queremos abandonar a hipótese de utilizarmos primeira aproximação linear, para concluirmos que $m_{\beta}^{-}$é 
estável em um espaço apropriado. Nesta direção veremos a seguir um resultado que nos garante que podemos restringir nosso estudo para um subconjunto compacto em $L^{2}(\mathbb{R}, \rho)$, em que a função $F$, que já era Hadamard diferenciável no mesmo, agora será também Fréchet diferenciável. Logo neste subconjunto, conseguimos utilizar primeira aproximação linear. Mais especificamente temos:

Teorema 3.2.9 Suponhamos $X$ e $Y$ espaços de Banach, $A \subset X$ um subconjunto aberto, $f: A \longrightarrow Y$ e $p \in A$. Se existe $X_{1} \subset X$ e a inclusão $i: X_{1} \hookrightarrow X$ é compacta, com $f$ Hadamard diferenciável em $p \in X_{1}$, então a composição $\tilde{f}=f \circ i$ é Fréchet diferenciável em $p$.

Demonstração: Seja $T=d f(p)$ a diferencial de Hadamard de $f$ em $p$, logo $T$ é um operador linear limitado. Uma vez que $i$ é compacta, então $i$ também é limitada (ver [26], pág. 406, Lema 8.1-2), assim $\tilde{T}=T \circ i: X_{1} \longrightarrow Y$ também é um operador linear limitado.

Usaremos, agora, a Proposição 2.1.10 para mostrarmos que $\tilde{T}$ é a diferencial de Fréchet de $\tilde{f}$ em $p \in X_{1}$, com $\|\cdot\|_{X_{1}}$ e $\|\cdot\|_{Y}$ denotando as normas em $X_{1}$ e $Y$, respectivamente. Suponhamos, por contradição, que exista uma seqüência $\left(t_{n}, h_{n}\right)$ em $\mathbb{R} \times X_{1}$ tal que $t_{n} \rightarrow 0,\left\|h_{n}\right\|_{X_{1}}=1$ e para algum $\epsilon>0$ e todo $n$ temos

$$
\left\|\frac{\tilde{f}\left(p+t_{n} h_{n}\right)-\tilde{f}(p)-t_{n} \tilde{T} h_{n}}{t_{n}}\right\|_{Y}>\epsilon .
$$

Uma vez que $i$ é compacta, então $i$ leva seqüências limitadas de $X_{1}$ sobre seqüências em $X$ que possuem subseqüências convergentes (ver [26], pág. 407, Teorema 8.1-3). Daí, existe uma subseqüência de $\left(i\left(h_{n}\right)\right)=\left(h_{n}\right)$, que também denotaremos por $\left(h_{n}\right)$, convergindo em $X$ para $h \in X$. Portanto,

$$
\begin{aligned}
& \left\|\frac{\tilde{f}\left(p+t_{n} h_{n}\right)-\tilde{f}(p)-t_{n} \tilde{T} h_{n}}{t_{n}}\right\|_{Y}=\left\|\frac{(f \circ i)\left(p+t_{n} h_{n}\right)-(f \circ i)(p)-t_{n}(T \circ i) h_{n}}{t_{n}}\right\|_{Y} \\
= & \left\|\frac{f\left(p+t_{n} h+t_{n}\left(h_{n}-h\right)\right)-f(p)-t_{n} T h-t_{n} T\left(h_{n}-h\right)}{t_{n}}\right\|_{Y} \\
\leqslant & \left\|\frac{f\left(p+t_{n} h+t_{n}\left(h_{n}-h\right)\right)-f(p)-t_{n} T h}{t_{n}}\right\|_{Y}+\left\|T\left(h_{n}-h\right)\right\|_{Y} .
\end{aligned}
$$

Como $f$ é Hadamard diferenciável em $p$, a primeira parcela tende a zero quando $t_{n} \rightarrow 0$ e $h_{n} \rightarrow h$. E ainda, $T$ é um operador limitado, logo $T\left(h_{n}-h\right) \rightarrow 0$ quando 
$h_{n} \rightarrow h$. Mas por (3.37) isto é um absurdo.

Então, será que existe um subconjunto $X_{1} \subset L^{2}(\mathbb{R}, \rho)$, com inclusão $i: X_{1} \hookrightarrow$ $L^{2}(\mathbb{R}, \rho)$ compacta (veremos mais adiante que este subconjunto sempre existe para qualquer espaço de Banach). De fato, este subconjunto existe e é o que veremos no teorema a seguir.

Teorema 3.2.10 O conjunto $X_{1}=\left\{u \in H^{1}(\mathbb{R}, \rho): \quad\|u\|_{\infty} \leqslant 1, \quad\|u\|_{H^{1}(\mathbb{R}, \rho)} \leqslant 1\right\}$ é subconjunto compacto de $L^{2}(\mathbb{R}, \rho)$.

Demonstração: Como $X_{1}$ é subconjunto limitado em $L^{2}(\mathbb{R}, \rho)$, para mostrarmos que este é compacto basta, pela Definição 2.6.1, que para todo $\epsilon>0$ a medida de Hausdorff $\gamma\left(X_{1}\right) \leqslant \epsilon$.

Seja $\epsilon>0$ e consideremos a função constante igual a um, definida sobre todo $\mathbb{R}$. Uma vez a função peso $\rho$ faz com a norma em $L^{2}(\mathbb{R}, \rho)$ das funções constantes, "fique pequena para grandes valores de $x "$, então é possível encontrarmos $\eta>0$ tal que

$$
\int_{-\infty}^{\eta} 1 \rho(x) d x+\int_{\eta}^{+\infty} 1 \rho(x) d x<\epsilon .
$$

Olhando agora para o intervalo $[-\eta, \eta]$, definimos a função característica

$$
\mathcal{X}_{\eta}(u)= \begin{cases}1, & \text { se } u \in[-\eta, \eta] \\ 0, & \text { se } u \notin[-\eta, \eta]\end{cases}
$$

Tomando $u \in X_{1}$, temos $u=\mathcal{X}_{\eta} u+\left(1-\mathcal{X}_{\eta}\right) u$, assim $X_{1}=A \cup B$, onde $A=$ $\left\{\mathcal{X}_{\eta} u: u \in X_{1}\right\}$ e $B=\left\{\left(1-\mathcal{X}_{\eta}\right) u: u \in X_{1}\right\}$. Inicialmente, afirmamos que $B$ está contido na bola aberta de centro na origem e raio $\epsilon$ em $L^{2}(\mathbb{R}, \rho)$, isto é, $B \subset B_{\epsilon}(0)$. De fato, para $x \in B$ temos $x=\left(1-\mathcal{X}_{\eta}\right) u$, com $u \in X_{1}$ e,

$$
\begin{aligned}
& \|x\|_{L^{2}(\mathbb{R}, \rho)}^{2}=\int_{\mathbb{R}}\left(1-\mathcal{X}_{\eta}\right)^{2} u^{2} \rho(x) d x \\
& =\int_{-\infty}^{-\eta}\left(1-\mathcal{X}_{\eta}\right)^{2} u^{2} \rho(x) d x+\int_{-\eta}^{\eta}\left(1-\mathcal{X}_{\eta}\right)^{2} u^{2} \rho(x) d x+\int_{\eta}^{+\infty}\left(1-\mathcal{X}_{\eta}\right)^{2} u^{2} \rho(x) d x \\
& <\int_{-\infty}^{-\eta}\|u\|_{\infty}^{2} \rho(x) d x+\int_{\eta}^{+\infty}\|u\|_{\infty}^{2} \rho(x) d x \leqslant \int_{-\infty}^{-\eta} \rho(x) d x+\int_{\eta}^{+\infty} \rho(x) d x<\epsilon .
\end{aligned}
$$

Logo, $x \in B_{\epsilon}(0)$. Assim, $\gamma(B) \leqslant \epsilon$. 
Agora, analisando $A$ como um subconjunto de $H^{1}([-\eta, \eta], \rho)$, segue que $A$ é limitado, uma vez que $\left\|\mathcal{X}_{\eta} u\right\|_{H^{1}([-\eta, \eta], \rho)} \leqslant 1$, e ainda é fechado. O espaço $H^{1}([-\eta, \eta], \rho)$ é compacto em $L^{2}([-\eta, \eta], \rho)$ (ver [37], pág. 238, Teorema 21.A), logo $A$ é compacto como subconjunto de $L^{2}([-\eta, \eta], \rho)$. Mas fora de $[-\eta, \eta]$, temos $A=\{0\}$, assim $A$ é compacto como subconjunto de $L^{2}(\mathbb{R}, \rho)$, portanto, pelo item (i) do Lema 2.6.2, $\gamma(A)=0$. Daí, por $(v)$ do Lema 2.6.2,

$$
\gamma\left(X_{1}\right)=\gamma(A \cup B)=\max \{\gamma(A), \gamma(B)\} \leqslant \epsilon .
$$

Como conseqüência dos Teoremas 3.2 .9 e 3.2 .10 podemos concluir que a função $F$, dada por (3.32), é Fréchet diferenciável em $X_{1}$. Uma vez, pelo Teorema 3.2.5, o espectro do operador $D F\left(m_{\beta}^{-}\right)$está localizado sobre o semi-plano esquerdo, então, pelos resultados da Subseção 3.1.1, o ponto de equilíbrio $m_{\beta}^{-}$é estável em $X_{1}$, e ainda podemos afirmar que $m_{\beta}^{-}$é assintoticamente estável em $X_{1}$.

\section{Observações:}

1. Notemos que, dado um espaço de Banach $X$ sempre é possível encontrarmos um subespaço $X_{1} \subset X$ compacto em $X$, basta tomarmos por exemplo $X_{1}=\{c v: c \in \mathbb{R}\}$, para qualquer $v \in X$, com a norma definida por $\|\cdot\|_{X_{1}}=|c|$.

2. Uma vez que o estudo da estabilidade, por primeira aproximação linear, das soluções de equilíbrio de uma equação diferencial em um espaço de Banach $X$, por exemplo da forma (3.29), depende essencialmente da norma definida neste espaço, pois para falarmos em equilíbrios estáveis precisamos, pela Definição 3.1.1, que exista, de certa forma, convergência na norma de $X$. O Teorema 3.2.9 nos diz que quando a função $f$ for apenas Hadamard diferenciável em $X$, sempre é possível restringirmos nosso estudo da estabilidade para um subespaço compacto em $X$ possuindo uma nova norma, além da norma definida em $X$, na qual temos convergência, e ainda a função $f$ é Fréchet diferenciável. Logo podemos obter estabilidade por primeira aproximação linear.

3. Com base no que vimos nesta seção podemos concluir que Hadamard diferenciabilidade não é suficiente para obtermos resultados do tipo Hartman-Grobman. Para mais detalhes, consultar [16]. 


\section{Apêndice}

\section{A.1 Elementos de Integração}

Definição A.1.1 Dizemos que uma família $\mathscr{X}$ de subconjuntos de um conjunto $X$ é uma $\sigma$-álgebra se satisfaz as seguintes propriedades:

(i) $\emptyset$ e $X$ pertencem a $\mathscr{X}$;

(ii) se $A$ pertence a $\mathscr{X}$, então o complementar $\mathscr{C}(A)=X \backslash A$ pertence a $\mathscr{X}$;

(iii) se $\left(A_{n}\right)$ é uma seqüência de conjuntos em $\mathscr{X}$, então $\bigcup_{n=1}^{\infty} A_{n}$ pertence a $\mathscr{X}$.

Definição A.1.2 Dizemos que um par ordenado $(X, \mathscr{X})$, consistindo de um conjunto $X$ e uma $\sigma$-álgebra $\mathscr{X}$ de subconjuntos de $X$, é um espaço mensurável. Chamamos qualquer conjunto em $\mathscr{X}$ de conjunto $\mathscr{X}$-mensurável, entretanto quando a $\sigma$-álgebra $\mathscr{X}$ é fixada, dizemos apenas que o conjunto é mensurável.

Observação: Tomando $X=\mathbb{R}$ na Definição A.1.1, obtemos a álgebra de Borel que é a $\sigma$-álgebra $\mathscr{B}$ gerada por todos intervalos abertos ou fechados em $\mathbb{R}$.

Definição A.1.3 Dizemos que uma função $f: X \longrightarrow \mathbb{R}$ é mensurável, se para cada número real $\alpha$ o conjunto $\{x \in X: f(x)>\alpha\}$ pertencer a $\mathscr{X}$.

Observação: Analisarmos se o conjunto $\{x \in X: f(x)>\alpha\}$ é mensurável é equivalente a analisarmos se qualquer um dos conjuntos obtidos quando trocamos o sinal ">" por " $\geqslant$ " ou "<" ou " $\leqslant$ " é mensurável (ver [3], pág. 8, Lema 2.4). 
Definição A.1.4 Uma medida é uma função $\mu$ definida sobre uma $\sigma$-álgebra $\mathscr{X}$ de subconjuntos de $X$ em $\mathbb{R}$ tal que $\mu(\emptyset)=0, \mu(E) \geqslant 0$ para todo $E \in \mathscr{X}$, e se $\left(E_{n}\right)$ é qualquer seqüência de conjuntos disjuntos em $\mathscr{X}$, então

$$
\mu\left(\bigcup_{n=1}^{\infty} E_{n}\right)=\sum_{n=1}^{\infty} \mu\left(E_{n}\right) .
$$

Definição A.1.5 Dizemos que $M \in \mathscr{X}$ tem medida $\mu$, nula, se $\mu(E \cap M)=0$ para todo $E \in \mathscr{X}$.

Observação: Se $X=\mathbb{R}$ e $\mathscr{X}=\mathscr{B}$, então existe uma única medida $\lambda$ definida sobre $\mathscr{B}$, que coincide com o comprimento dos intervalos. Neste caso, $\lambda$ é chamada de medida de Lebesgue.

Definição A.1.6 Dizemos que uma proposição vale em quase todo lugar, se existe um conjunto $M \in \mathscr{X}$, com medida $\mu(M)=0$ tal que a proposição vale no complementar de $M$.

Para mais detalhes das definições dadas acima, consultar [3] e [19].

Definição A.1.7 Definimos a oscilação de uma função $x:[a, b] \longrightarrow \mathbb{R}$ por

$$
\omega_{x}([a, b])=\sup \left\{\left|x\left(t_{1}\right)-x\left(t_{2}\right)\right|: t_{1}, t_{2} \in[a, b]\right\} .
$$

Definição A.1.8 Dizemos que uma função $x:[a, b] \longrightarrow \mathbb{R}$ é absolutamente contínua $(A C)$, se para todo $\epsilon>0$, existe um $\delta=\delta(\epsilon)>0$ tal que se $\left\{\left[a_{i}, b_{i}\right]: i=1, \ldots, n\right\}$ é qualquer subpartição de $[a, b]$ tal que $\sum_{i=1}^{n}\left|b_{i}-a_{i}\right| \leqslant \delta$, então $\sum_{i=1}^{n}\left|x\left(b_{i}\right)-x\left(a_{i}\right)\right| \leqslant$ $\epsilon$. E ainda, dizemos que $x$ é absolutamente contínua no sentido restrito $\left(A C_{*}\right)$, se $x$ é limitada sobre um intervalo que contém $[a, b]$ e, para todo $\epsilon>0$, existe $\delta>0$ tal que $\sum_{i=1}^{n} \omega_{x}\left(\left[a_{i}, b_{i}\right]\right)<\epsilon$, para qualquer subpartição $\left\{\left[a_{i}, b_{i}\right]: i=1, \ldots, n\right\}$ de $[a, b]$ tal que $\sum_{i=1}^{n}\left|b_{i}-a_{i}\right|<\delta$.

\section{Observações:}

1. Se uma função $x:[a, b] \longrightarrow \mathbb{R}$ é $A C$, então é uniformemente contínua sobre $[a, b]$. De fato, tomemos $t_{0} \in[a, b]$. Dado $\epsilon>0$, existe $\delta=\delta(\epsilon)>0$, como na Definição A.1.8. Se $t \in[a, b]$ e $\left|t-t_{0}\right| \leqslant \delta$, então $\left|x(t)-x\left(t_{0}\right)\right| \leqslant \epsilon$. Assim $x$ é contínua em um ponto arbitrário $t_{0} \in[a, b]$. Uma vez que $\delta$ não depende de $t_{0}$, a função $x$ é uniformemente contínua.

2. Se $x:[a, b] \longrightarrow \mathbb{R}$ é $A C$, então é diferenciável em quase todo $t \in[a, b]$. 
Definição A.1.9 Dizemos que uma função $x:[a, b] \longrightarrow \mathbb{R}$ é absolutamente contínua generalizada (ACG) sobre $[a, b]$, se $x$ é contínua sobre $[a, b]$ e se existe uma seqüência enumerável $\left(I_{n}\right)_{n \geqslant 1}$ de intervalos em $[a, b]$ tal que $[a, b]=\bigcup_{n=1}^{\infty} I_{n}$ e $x$ é AC sobre cada $I_{n}$, para todo $n$. E ainda, dizemos que $x$ é absolutamente contínua generalizada no sentido restrito $\left(A C G_{*}\right)$ sobre $[a, b]$, se $x$ é contínua sobre $[a, b]$ e $[a, b]$ pode ser escrito como uma união enumerável de intervalos, sobre cada qual $x$ é $A C_{*}$.

O estudo das funções dadas nas Definições A.1.8 e A.1.9 pode ser encontrado em [2] e [19].

\section{A.2 O Espectro de um Operador Linear}

Sejam $X \neq\{0\}$ um espaço normado complexo e $A$ um operador linear com domínio $D(A)$ e imagem $\operatorname{Im}(A)$ contidos em $X$. Para motivarmos a definição de espectro, relembremos o conceito de autovalor. Um escalar $\lambda$ é um autovalor para $A$ se, e somente se, o operador linear $(\lambda I-A)$ não é injetor, isto é, se, e somente se, o auto-espaço $N(\lambda I-A)$ (núcleo de $(\lambda I-A)$ ) correspondente ao autovalor $\lambda$ é não trivial. Se $(\lambda I-A)$ não é injetor, então não é invertível. Logo $(\lambda I-A)$ não tem uma inversa definida sobre sua imagem. Agora, pode ser que $(\lambda I-A)$ seja injetor, isto é, $\lambda$ não é um autovalor e $(\lambda I-A)$, todavia, não tem uma inversa definida sobre $X$. Ou, topologicamente, a inversa pode existir mas não ser contínua. De fato, existe um número de possibilidades para $(\lambda I-A)$, isto sugere que uma forma de analisarmos um operador linear $A$ é estudarmos os $\lambda$ 's para o qual $(\lambda I-A)$ deixa de ter uma inversa contínua. Este subconjunto de $\lambda$ 's chamamos de espectro de $A$.

Definição A.2.1 Seja $A: D(A) \longrightarrow X$ um operador linear com dominio $D(A) \subset X$. $O$ conjunto de todos os números complexos $\lambda$ tais que a imagem de $(\lambda I-A)$ é densa em $X$ e tal que $(\lambda I-A)$ tem uma inversa contínua definida sobre sua imagem definimos como sendo o conjunto resolvente de $A$ e denotamos por $\rho(A)$. O conjunto de todos os números complexos que não estão no conjunto resolvente definimos como sendo o espectro de $A$ e denotamos por $\sigma(A)$.

Existem vários caminhos para que um número complexo $\lambda$ não esteja no conjunto resolvente $\rho(A)$. Estes fatos nos direciona para a seguinte subdivisão do espectro $\sigma(A)$ : 
(i) o espectro pontual de um operador linear $A$ é o subconjunto de todos os $\lambda$ 's em que o operador $(\lambda I-A)$ não é injetor. Isto é, o espectro pontual é exatamente o conjunto de todos autovalores. Denotamos por $\sigma_{p}(A)$;

(ii) o espectro contínuo de um operador linear $A$ é o subconjunto de todos os $\lambda$ 's em que o operador $(\lambda I-A)$ tem sua imagem densa em $X$, é injetor e a inversa definida sobre a imagem não é contínua. Denotamos por $\sigma_{c}(A)$;

(iii) o espectro residual de um operador linear $A$ é o subconjunto de todos os $\lambda$ 's em que o operador $(\lambda I-A)$ é injetor mas não tem sua imagem densa em $X$. Denotamos por $\sigma_{r}(A)$.

\section{Observações:}

1. Os conjuntos $\sigma_{p}(A), \sigma_{c}(A)$ e $\sigma_{r}(A)$ são disjuntos e $\sigma(A)=\sigma_{p}(A) \cup \sigma_{c}(A) \cup \sigma_{r}(A)$.

2. Se $X$ tem dimensão finita então $\sigma(A)=\sigma_{p}(A)$. Para ver a demonstração desta afirmação, consultar [31], pág. 414, Exemplo 1.

Suponhamos, agora, que $X$ seja um espaço de Banach e denotemos por $\mathcal{B}(X)$ o espaço de Banach de todos os operadores lineares limitados $A: X \longrightarrow X$, com a norma $\|A\|=\sup _{\|x\| \leqslant 1}\|A x\|$. Nestas condições, temos a seguinte propriedade do espectro de um operador $A \in \mathcal{B}(X)$ :

Teorema A.2.2 Seja $A \in \mathcal{B}(X)$. Se $\|A\|<1$ então $(I-A)^{-1}$ existe e é um operador limitado. E ainda

$$
(I-A)^{-1}=I+A+A^{2}+\ldots=\sum_{n=0}^{\infty} A^{n},
$$

onde a série é convergente na norma sobre $\mathcal{B}(X)$, e $\left\|(I-A)^{-1}\right\| \leqslant(1-\|A\|)^{-1}$.

Demonstração: Ver [31], pág. 431, Teorema 6.7.1.

\section{A.3 Exponencial de um Operador Linear Limitado}

$\mathrm{Na}$ teoria de Equações Diferenciais um papel importante é desempenhado pela exponencial de um operador $e^{A t}$, onde $A \in \mathcal{B}(X)$. Para definirmos $e^{A t}$ consideremos 
inicialmente a série

$$
\sum_{n=0}^{\infty} \frac{(A t)^{n}}{n !}
$$

O lado direito de (A.1) converge em norma para todo $t \geqslant 0$. De fato, seja

$$
S_{k}=\sum_{n=0}^{k} \frac{(A t)^{n}}{n !}
$$

Para $m<k$ temos

$$
\left\|S_{k}-S_{m}\right\|=\left\|\sum_{n=0}^{k} \frac{(A t)^{n}}{n !}-\sum_{n=0}^{m} \frac{(A t)^{n}}{n !}\right\|=\left\|\sum_{n=m+1}^{k} \frac{(A t)^{n}}{n !}\right\| \leqslant \sum_{n=m+1}^{k} \frac{\|A\|^{n}|t|^{n}}{n !} \rightarrow 0
$$

quando $k, m \rightarrow \infty$, pois $e^{\|A\||t|}$ converge em $\mathbb{R}$. Então $\left(S_{k}\right)$ é uma seqüência de Cauchy no espaço de Banach dos operadores lineares limitados $\mathcal{B}(X)$, que é completo, e assim converge para um elemento em $\mathcal{B}(X)$, que denotaremos por $e^{t A}$. Então podemos definir

$$
e^{A t}=\sum_{n=0}^{\infty} \frac{A^{n} t^{n}}{n !}
$$

Uma outra maneira de definirmos $e^{A t}$ é por meio da seguinte relação

$$
e^{A t}=\frac{1}{2 \pi \imath} \int_{\Gamma_{A}} e^{\lambda t} R(\lambda: A) d \lambda
$$

onde $\Gamma_{A}$ é um contorno ao redor do espectro $\sigma(A)$ de $A$, percorrido no sentido positivo, e $R(\lambda: A)$ é o valor do resolvente do operador linear limitado $A$ em $\lambda$, isto é, $R(\lambda: A)=(\lambda I-A)^{-1}$.

O operador $e^{A t}$ definido por (A.2) ou (A.3) tem a propriedade de grupo a um parâmetro, isto é, $e^{A t} e^{A s}=e^{A(t+s)},-\infty<t, s<\infty$ e $\left.e^{A t}\right|_{t=0}=I_{d}$, onde $I_{d}$ é o operador identidade em $X$. Temos também a seguinte propriedade

$$
\frac{d}{d t} e^{A t}=e^{A t} A=A e^{A t},
$$

que é de grande importância na teoria das Equações Diferenciais. A demonstração de (A.4) pode ser encontrada, por exemplo, em [7], pág. 25.

E ainda,

$$
\left\|e^{A t}\right\| \leqslant \sum_{n=0}^{\infty} \frac{\left\|A^{n}\right\| t^{n}}{n !}=e^{\|A\| t}, \quad t \geqslant 0
$$




\section{Referências Bibliográficas}

[1] BANAŚ, J.; GOEBEL, K. Measures of Noncompactness in Banach Spaces. New York; Basel: Marcel Dekker, 1980. (Lecture Notes in Pure and Applied Mathematics, v. 60$)$

[2] BARTLE, R. G. A Modern Theory of Integration. Providence: American Mathematical Society, 2001. (Graduate Studies in Mathematics, v. 32)

[3] BARTLE, R. G. The Elements of Integration. New York: John Wiley \& Sons, 1966.

[4] BLASI, F. S.; PIANIGIANI, G. Uniqueness for Differential Equations Implies Continuous Dependence only in Finite Dimension. Bulletim of the London Mathematical Society, v. 18, n. 73, p. 379-382, 1986.

[5] CICHON, M. On Solutions of Differential Equations in Banach Spaces. Nonlinear Analysis, v. 60, n. 4, p. 651-667, 2005.

[6] CICHOŃ, M.; KUBIACZYK, I.; SIKORSKA, A. The Henstock-Kurzweil-Pettis Integral and Existence Theorems for the Cauchy Problem. Czechoslovak Mathematical Journal, v. 54, n. 129, p. 279-289, 2004.

[7] DALECKIĨ, J. L.; KREĨN, S. G. Stability of Solutions of Differential Equations in Banach Space. Providence: American Mathematical Society, 1974. (Translations of Mathematical Monographs, v. 43)

[8] DAY, M. M. On the Basis Problem in Normed Spaces. Proceedings of the American Mathematical Society, v. 13, n. 4, p. 655-658, 1962. 
[9] DE MASI, A.; GOBRON, T.; PRESUTTI, E. Travelling Fronts in Non-Local Evolution Equations. Archive for Rational Mechanics and Analysis, v. 132, n. 2, p. 143-205, 1995.

[10] DE MASI, A.; ORLANDI, E.; PRESUTTI, E.; TRIOLO, L. Uniqueness and Global Stability of the Instanton in Non-Local Evolution Equations. Rendiconti di Matematica, v. 14, série 7, fasc. 4, p. 693-723, 1994.

[11] DEIMLING, K. Ordinary Differential Equations in Banach Spaces. New York: Springer, 1977. (Lecture Notes in Mathematics, v. 596)

[12] DIEUDONNÉ, J. Deux Exemples Singuliers D'Équations Difféntielles. Acta Scientiarum Mathematicarum (Szeged), v. 12, p. 38-40, 1950.

[13] DUGUNDJI, J. Topology. Boston: Allyn; Bacon, 1966.

[14] DUNFORD, N.; SCHWARTZ, J. T. Linear Operators-Parte I: General Theory. New York: John Wiley \& Sons, 1988.

[15] FEDERSON, M. C. The Fundamental Theorem of Calculus for Multidimensional Banach Space-Valued Henstock Vector Integrals. Real Analysis Exchange, v. 25, n. 1, p. 469-480, 1999-2000.

[16] FICHMANN, L. A Compact Dissipative Dynamical System for a Difference Equation with Diffusion. Journal of Differential Equations, v. 138, n. 1, p. 1-18, 1997.

[17] FLETT, T. M. Differential Analysis. New York: Cambridge University, 1980.

[18] GODUNOV, A. N. Peano's Theorem in Banach Spaces. Functional Analysis and Applications, v. 9, n. 1, p. 53-55, 1975.

[19] GORDON, R. A. The Integrals of Lebesgue, Denjoy, Perron, and Henstock. Providence: American Mathematical Society, 1994. (Graduate Studies in Mathematics, v. 4)

[20] HALE, J. K. Ordinary Differential Equations. New York: John Wiley \& Sons, 1969. (Pure and Applied Mathematics, v. 21) 
[21] HARTMAN, P. Ordinary Differential Equations. New York: John Wiley \& Sons, 1964 .

[22] HENRY, D. B. Differential Calculus in Banach Space. São Paulo: S.N., 2000. (Notas de Aula)

[23] HENRY, D. B. Geometric Theory of Semilinear Parabolic Equations. Berlin: Springer, 1981. (Lecture Notes in Mathematics, v. 840)

[24] HÖNIG, C. S. Análise Funcional e Aplicações. v. 1. São Paulo: IME-USP, 1970.

[25] KREĨN, S. G. Linear Differential Equations in Banach Space. Providence: American Mathematical Society, 1971. (Translations of Mathematical Monographs, v. 29)

[26] KREYSZIG, E. Introductory Functional Analysis with Aplications. New York: John Wiley \& Sons, 1978.

[27] LADAS, G. E.; LAKSHMIKANTHAM, V. Differential Equations in Abstract Spaces. New York: Academic Press, 1972. (Mathematics in Science and Engineering, v. 85)

[28] LIMA, E. L. Curso de Análise. v.1. 5.ed. Rio de Janeiro: CNPq, 1976.

[29] MIKUSIŃSKI, J. The Bochner Integral. Switzerland: Birkhäuser, 1978.

[30] NASHED, M. Z. Differentiability and Related Properties of Nonlinear Operators: Some Aspects of the Role of Differentials in Nonlinear Functional Analysis. IN RALL, L. B. Nonlinear Functional Analysis and Applications. New York: Academic Press, 1971.

[31] NAYLOR, A. W.; SELL, G. R. Linear Operator Theory in Engineering and Science. New York: Springer, 1982.

[32] PAZY, A. Semigroups of Linear Operators and Applications to Partial Differential Equations. New York: Springer, 1983.

[33] PENG-YEE, L. Lanzhou Lectures on Henstock Integration. Singapore: World Scientific Publishing, 1989. (Series in Real Analysis, v. 2) 
[34] PEREIRA, A. L. Global Attractor and Non Homogeneous Equilibria for a Non Local Evolution Equation in an Unbounded Domain. (Preprint)

[35] SCHÄFFER, J. J. Uniqueness without Continuous Dependence in Infinite Dimension. Journal of Differential Equations, v. 56, n. 1, p. 426-428, 1985.

[36] SZÉP, A. Existence Theorem for Weak Solutions of Ordinary Differential Equations in Reflexive Banach Spaces. Studia Scientiarum Mathematicarum Hungarica, v. 6, fasc. 1-2, p. 197-203, 1971.

[37] ZEIDLER, E. Nonlinear Functional Analysis and its Applications-Parte II/A: Linear Monotone Operators. New York: Springer, 1990.

[38] YORKE, J. A. A Continuous Differential Equations in Hilbert Space without Existence. Funkcialaj Ekvacioj, v. 13, n. 3, p. 19-21, 1970.

[39] YOSIDA, K. Functional Analysis. 6. ed. New York: Springer, 1980. 